\title{
Crystallisation of metamorphic garnet: mechanisms and rates from the inverted Barrovian sequence in the Sikkim Himalaya
}

\author{
by \\ Freya Roxanne George
}

\begin{abstract}
A dissertation submitted to the
Faculty of Graduate and Postdoctoral Affairs

in partial fulfillment of the requirements for the degree of
\end{abstract}

Doctor of Philosophy in Earth Science

Ottawa-Carleton Geoscience Centre

Department of Earth Sciences

Carleton University

Ottawa, Ontario

May 2019 


\section{C)Copyright}

Freya Roxanne George, 2019 


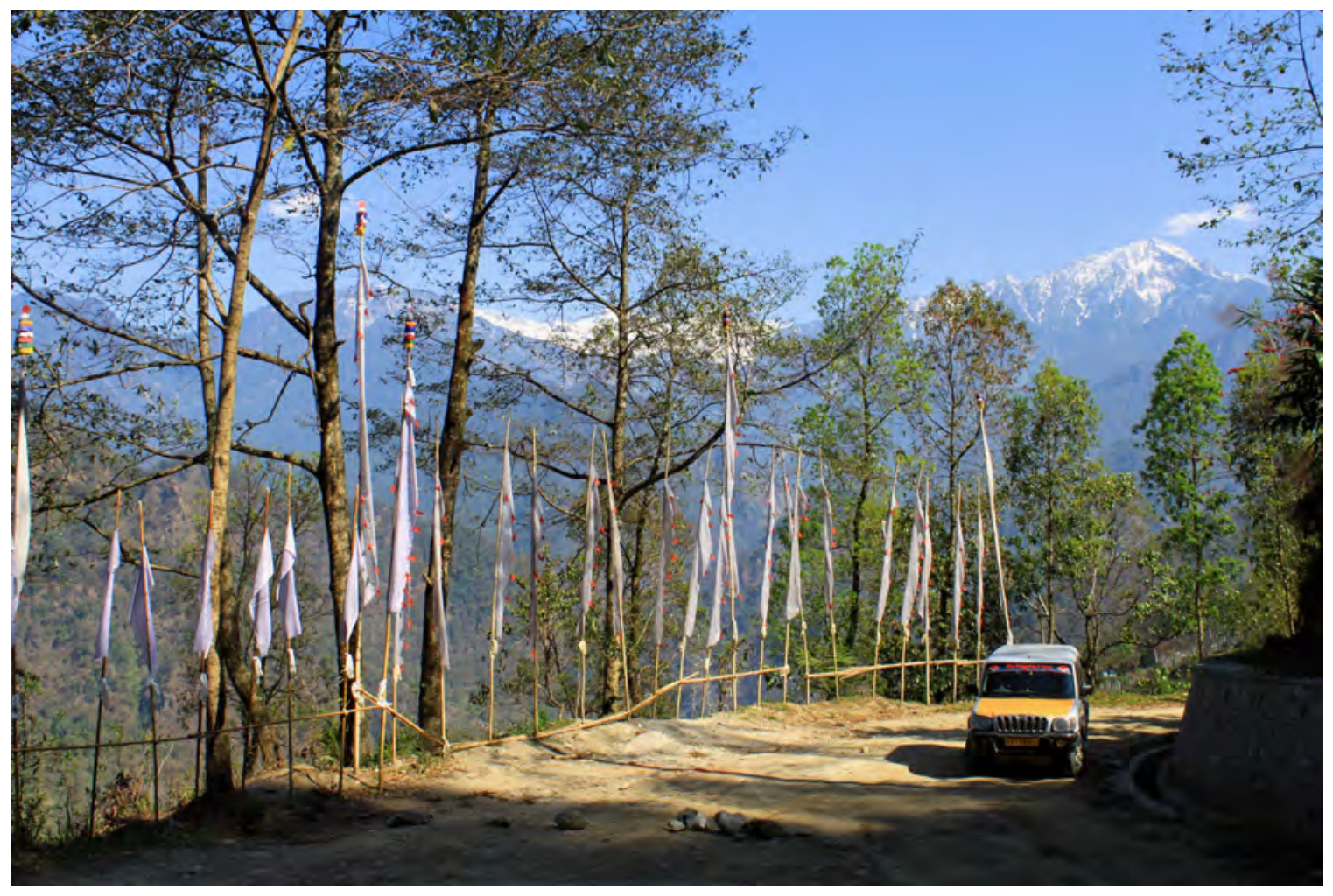

If we knew what we were doing, it wouldn't be called research. - Albert Einstein 


\section{Abstract}

The compositional zonation, three-dimensional spatial distribution, and morphologies of a metamorphic garnet population represent an integrated record of the population's nucleation and growth history over changing metamorphic conditions. While previous inferences drawn from such datasets have proven diverse, their continued collection is key to better understanding the mechanisms and processes that control porphyroblast crystallisation, rates of intergranular element transfer, and the extent to which chemical equilibrium persists across a rock volume during metamorphism. This thesis utilises such datasets from the inverted Barrovian metamorphic sequence in the Sikkim Himalaya, India, where the record of recent orogenesis provides an ideal natural laboratory in which fundamental rock-forming processes can be investigated.

Compositional analyses of a representative population of garnet porphyroblasts from a garnet-grade metapelite indicate a strong correlation between garnet crystal size and composition with respect to both major end-member and trace element components. Numerical simulation of progressive nucleation and growth using an equilibrium approach and multicomponent diffusion reproduces the sizes and major element zoning of the entire garnet population along a rapid heating trajectory $\left(>100{ }^{\circ} \mathrm{C} \mathrm{Myr}^{-1}\right.$ ). Given the correspondingly rapid rates of garnet growth $\left(\sim 1.4 \mathrm{~mm} \mathrm{Myr}^{-1}\right)$ and negligible departure from equilibrium, major element transport is inferred to have been non-limiting, with growth rates controlled by interfacial processes. 
In contrast, trace element zoning in the same population is indicative of persistent disequilibrium with respect to rare-earth elements, yttrium and chromium. Oscillatory zoning may reflect incorporation of chemical heterogeneities and minor fluctuations in the garnet growth rate not resolvable in major element zoning, and annuli are not demonstrably relatable to some rock-wide event. Elements that do not equilibrate across garnet surfaces document continuous spiral zoning, which permits estimation of syn-crystallisation strain-rates, on the order of $10^{-11}-10^{-12} \mathrm{~s}^{-1}$.

Microstructural data from across the Barrovian sequence reveal garnet populations that are partially characterised by their grade, with changing crystal size and abundance interpreted to reflect the combined effects of bulk chemical variations and ripening with increasing pressures and temperatures. Deviations from this trend reflect populations in which garnet crystallisation was controlled by a highly heterogeneous spatial distribution of nucleation sites and transport-limited growth. 


\section{Acknowledgements}

First and foremost, my biggest debt of gratitude goes to my supervisor, Fred Gaidies. Your guidance, patience, and willingness to let me go my own route (but steering me back on course when needed) has been invaluable, and I've no doubt I'll continue to learn from you in the future. I'm honoured to have been your first $\mathrm{PhD}$ student.

The basis of this thesis is in detailed analytical work that would have been impossible without the dedicated support of lab technicians and support staff. To Peter Jones, thank you for the endless hours of microprobe time and giving me a little glimpse into the 'Good Old Days' of the Carleton Earth Sciences Department; this work would not have been possible without you. To Glenn Poirer, thank you for the maps and fitting me into the busy analytical schedule. Next, thanks to Brandon Boucher and Christopher McFarlane at the University of New Brunswick for help collecting our beautiful laser maps (if I ever get them made into t-shirts, they're on me), and particularly to Brandon for putting up with my endless emails about reprocessing. I am indebted to Lars Hansen at the University of Oxford for EBSD support and instrumentation, and Agatha Doboz of Queen's University is thanked for providing access to the SelFrag instrumentation. Tim Mount is thanked for the production of many thin sections in this study and the insightful discussions about sample preparation (and his continued and much appreciated kindness). I will be forever grateful to Claudia Schröder-Adams, who has helped me keep my eyes on the finish line. To

all of the administrative staff in the Carleton Earth Sciences Department (especially 
Sheila and Beth), thank you for putting up with my endless questions and requests.

Financial support from Trillium, OGS, Carleton University, the Geological Society of America, the Walker Mineralogical Society and numerous internal grants has been invaluable. I would not have had half the opportunities I've experienced during this process without it. Sumit Chakraborty, Dave Pattison and Frank Spear are thanked for formal reviews of Chapter 2, and Jay Ague and Robert Anczkiewicz are thanked for formal reviews of Chapter 3.

I have seen many amazing graduate students pass through Carleton in my time here, all of whom have played a part in my $\mathrm{PhD}$ - thanks to you all. To all of the metamorphic grad students I've shared offices with, thank you for the stimulating discussions, putting up with my complaining, and for not dobbing me in when I nap at my desk. In particular to Arianne Petley-Ragan (our time together was alas, so short, but we've been fortunate to cover many miles) and Wilder Greenman (you've sold me on sedimentology, I will begrudgingly admit) your friendship has made my cumulative time in Ottawa very special. To the 'Bad Bitchez': Charlene Duffett, Kate Little, Alex Laudadio, and Sarah Davey, you have held me up this past year, let me be happy and sad and mad, and I consider myself incredibly lucky to have you all as friends and confidants. Extra-special thanks must go to Sarah, for putting up with me in even more concentrated doses as my sole Ottawa roommate, and helping with concept development for 'Bedside Wine ${ }^{\mathrm{TM}}$.' I am also indebted to all of my nongeologist friends, both in Canada and the UK, who both put up with my rock-chat, and provide some respite when required.

Lastly and most importantly, to my family: your unwavering and unconditional support truly means more to me than I could ever describe, and I couldn't have done any of this without you. You have kept me going through it all, and I owe everything to you. Thank you. 


\section{Preface}

This thesis is presented as three primary research chapters (Chapters 2-4), which are drawn directly from manuscripts that are published or are in preparation at the time of thesis submission. Each chapter is structured as a standalone manuscript with an abstract, introduction, methods and results section, discussions and conclusions, and supplementary data that form the appendices of this thesis. As a result of this formatting choice, there is some repetition, specifically pertaining to regional geology and methodology sections.

The copyright for most figures and text in Chapters 2 and 3 is held by Springer Nature, and permissions for included aspects of published chapters are presented in Appendix 1. Changes have been made to the published versions as follows: (1) figures are expanded and added to Chapter 2 to improve clarity; (2) supplementary data

included in Chapter 3 has been incorporated into the manuscript; (3) implications of trace element data for geochronology are included in Chapter 3, but not incorporated into the published manuscript.

The original citations for Chapters 2 and 3 are presented below.

Chapter 2: Major element zoning in garnet. Published as: George, F.R. and Gaidies, F., 2017. Characterisation of a garnet population from the Sikkim Himalaya: 
insights into the rates and mechanisms of porphyroblast crystallisation. Contributions to Mineralogy and Petrology, 172(7), p.57. (doi: 10.1007/s00410-017-1372-y). Intext references to this chapter are denoted by George \& Gaidies (2017)

Chapter 3: Trace element zoning in garnet. Published as: George, F.R., Gaidies, F., Boucher. B. 2018. Population-wide garnet growth zoning revealed by LA-ICP-MS mapping: implications for trace element equilibration and syn-kinematic deformation during crystallisation Contributions to Mineralogy and Petrology, 173(9), p.74. (doi: 10.1007/s00410-018-1503-0). In-text references to this chapter are denoted by George et al. (2018).

Chapter 4 is presented as a pre-submisson draft that is in the process of being adapted for submission to Journal of Metamorphic Geology.

In Chapter 2, the first author collected all the electron microprobe data (with the technical assistance of P. Jones and G. Poirer at Carleton University and University of Ottawa, respectively), conducted all thermobarometric and crystallisation modelling, drafted figures and wrote the manuscript. In Chapter 3, laser ablation-inductively coupled-mass spectrometry data was collected and processed with B. Boucher and C. McFarlane at the University of New Brunswick, and field-emission electron probe microanalysis was supported by T. Ntaflos and F. Kiraly at the University of Vienna. Data processing, interpretation and manuscript preparation was conducted by the first author. In Chapter 4, L. Hansen at the University of Oxford supervised electron back-scatter diffraction data collection, and G. Poirer assisted with collection of compositional analyses. The first author completed data processing, interpretation, presentation and the manuscript writing. In all research chapters, the second author 
and project supervisor, F. Gaidies, has been integral to project development, interpretation support, and manuscript editing. Numerical models utilised in Chapter 2 were developed by F. Gaidies and are detailed in Gaidies et al. (2008) and Gaidies et al. (2011).

Signed statements of co-authorship for Chapters 2 and 3 of this thesis are also included in Appendix 1, given that they have been drawn from papers that have been published in the aforementioned journals. 


\section{Contents}

Frontispiece i

Abstract ii

Acknowledgements iv

Preface vi vi vi ver

Table of Contents xii

$\begin{array}{ll}\text { List of Figures } & \text { XV }\end{array}$

List of Tables $\quad$ xvi

$\begin{array}{lll}1 & \text { Introduction } & 1\end{array}$

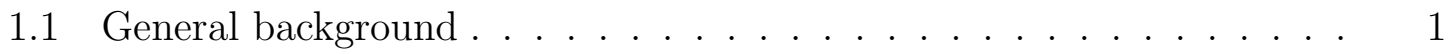

1.2 Metamorphic crystallisation . . . . . . . . . . . . . 5

1.2.1 Nucleation . . . . . . . . . . . . . . . . 5

1.2 .2 Growth . . . . . . . . . . . . . . . . . . 7

1.2 .3 Diffusion . . . . . . . . . . . . . . . . . . . . . . . . 10

1.3 The Sikkim Himalaya . . . . . . . . . . . . . . . . . . . . . . . . . . . 13

1.3 .1 Sample collection . . . . . . . . . . . . . . . 16

1.4 Thesis objectives $\ldots \ldots \ldots \ldots$. . . . . . . . . . . . . . . . 18 
1.5 Thesis structure $\ldots \ldots \ldots \ldots \ldots$

2 Major element zonation of a garnet population: rates and mecha$\begin{array}{ll}\text { nisms of crystallisation } & 20\end{array}$

2.1 Introduction . . . . . . . . . . . . . . . . . . . . . . . 20

2.1 .1 Crystallisation processes $\ldots \ldots \ldots \ldots \ldots$

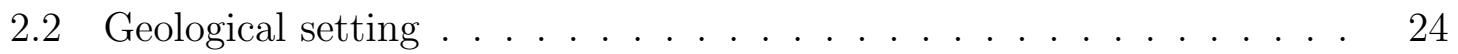

2.3 Textural and compositional characterisation . . . . . . . . . 25

2.3 .1 Methods . . . . . . . . . . . . . . . . . . 25

2.3 .2 Results . . . . . . . . . . . . . . . . . . . . . 28

$2.4 \quad$ Numerical simulations of garnet crystallisation $\ldots \ldots \ldots \ldots$

2.4 .1 Nucleation of garnet . . . . . . . . . . . . . . . . . 34

2.4 .2 Nucleation and growth of garnet . . . . . . . . . . . . 36

2.5 Discussion $\ldots \ldots \ldots \ldots \ldots \ldots$

2.5 .1 The rate of metamorphism . . . . . . . . . . . . 44

2.5 .2 Departure from equilibrium during garnet crystallisation . . . 45

$2.5 .3 \quad$ Effect of model assumptions . . . . . . . . . . . . . 47

2.5 .4 Processes controlling garnet growth $\ldots \ldots \ldots \ldots$

2.6 Conclusions $\ldots \ldots \ldots \ldots \ldots$

Tables and Figures $\ldots \ldots \ldots \ldots \ldots \ldots \ldots$

3 Trace element zonation of a garnet population: length scales of equi$\begin{array}{ll}\text { libration and deformation during crystallisation } & 70\end{array}$

3.1 Introduction . . . . . . . . . . . . . . . . . . . . . . . . . 70

3.2 Regional context and sample description $\ldots \ldots \ldots \ldots \ldots \ldots$

3.3 Methods . . . . . . . . . . . . . . . . . . . . . . 75

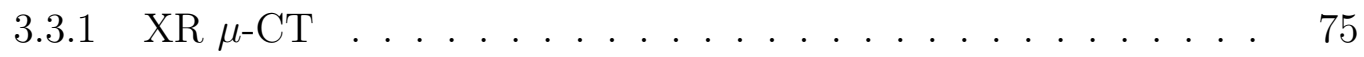


3.3 .2 Field-emission EPMA $\ldots \ldots \ldots \ldots \ldots$

$3.3 .3 \quad \mathrm{LA}-\mathrm{ICP}-\mathrm{MS} . \ldots \ldots \ldots \ldots \ldots$

3.4 Results . . . . . . . . . . . . . . . . . . . . . . . . . . . 77

3.4 .1 Garnet chemical composition . . . . . . . . . . . . 77

3.4 .2 Matrix phase geochemistry . . . . . . . . . . . . . . . 82

3.5 Discussion $\ldots \ldots \ldots \ldots \ldots$

$3.5 .1 \quad$ Origins of trace element distribution $\ldots \ldots \ldots$. . . . . 83

3.5 .2 Influence of rock texture on chemical zoning $\ldots \ldots \ldots$. . . 96

3.5 .3 Kinematics of porphyroblast rotation . . . . . . . . . . 97

3.5 .4 Implications for garnet geochronology . . . . . . . . . . . . . 101

3.6 Conclusions $\ldots \ldots \ldots \ldots$

Tables and Figures $\ldots \ldots \ldots \ldots \ldots$

4 Porphyroblast microstructure: sequence continuity and controls on $\begin{array}{ll}\text { irregular morphologies } & 123\end{array}$

4.1 Introduction . . . . . . . . . . . . . . . . . . . . . . . . . . . 123

$4.2 \quad$ Geological setting \& sample locations . . . . . . . . . . . . . . 125

4.3 Analytical methods . . . . . . . . . . . . . . . . . 126

$4.3 .1 \quad$ X-Ray $\mu$-computed tomography . . . . . . . . . . . . . 126

4.3 .2 Whole-rock and mineral major element chemical analyses . . . 127

$4.3 .3 \quad$ Electron backscatter diffraction $\ldots \ldots \ldots \ldots$

$4.3 .4 \quad$ Metamorphic phase equilibria modelling . . . . . . . . . . 129

$4.4 \quad$ Petrographic and chemical characterisation of samples . . . . . . . . 130

$4.4 .1 \quad$ Petrography and mineral chemistry . . . . . . . . . . . 130

4.4 .2 Whole-rock chemistry . . . . . . . . . . . . . . . . . 139

4.4 .3 Summary . . . . . . . . . . . . . . . . . . . . . 140 
4.5 Crystallographic characterisation of coalesced garnet using EBSD . . 142

$4.5 .1 \quad$ Epitaxial relationships . . . . . . . . . . . . . . . . 145

4.6 Metamorphic conditions of garnet crystallisation . . . . . . . . . . . . 148

4.6 .1 Crystallisation of isolated porphyroblastic garnet . . . . . . . 149

4.6 .2 Crystallisation of irregular garnet in SIM sample. . . . . . . . 152

4.7 Implications $\ldots \ldots \ldots \ldots$. . . . . . . . . . . . . . . . . . 153

4.7 .1 Polycrystal development in SIM3 . . . . . . . . . . . . . . . 153

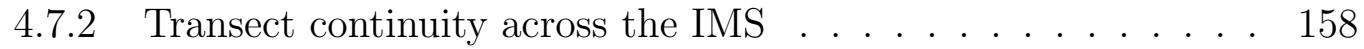

4.7 .3 Grade-related texture variations . . . . . . . . . . . . . . . . 161

4.8 Discussion of polycrystalline microstructures in SIM samples . . . . . 164

4.8.1 Bulk chemical effects on relative timing of porphyroblast nucleation in Sikkim . . . . . . . . . . . . . . . . 165

4.8 .2 Controls on heterogeneous garnet distribution . . . . . . . . . 167

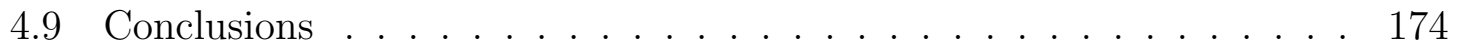

Tables and Figures . . . . . . . . . . . . . . . . . . . . . . 177

$\begin{array}{lll}5 & \text { Summary and Conclusions } & 201\end{array}$

5.1 Potential avenues of future work . . . . . . . . . . . . . . . . . 205

\begin{tabular}{ll}
\hline Bibliography & 235
\end{tabular}

\begin{tabular}{|l|l|}
\hline Appendices 1: Copyright and permissions & 236
\end{tabular}

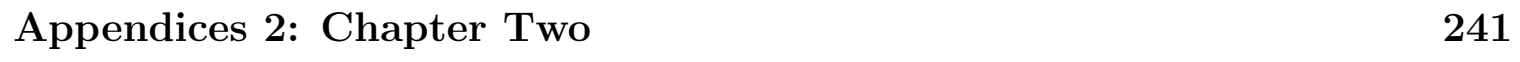




\section{List of Figures}

1.1 Gibbs energy of cluster formation $\ldots \ldots \ldots \ldots$

1.2 Chemical potential gradients $\ldots \ldots \ldots \ldots \ldots$

1.3 Himalayan tectonic map $\ldots \ldots \ldots \ldots$

1.4 Geology of Sikkim Himalaya . . . . . . . . . . . . . . . . . . . . 14

1.5 Sample localities $\ldots \ldots \ldots \ldots \ldots \ldots$

2.1 Geological map of the Sikkim Himalaya . . . . . . . . . . . . . 56

$2.2 \quad$ Photomicrographs representative of sample 24-99 $\ldots \ldots \ldots \ldots \ldots$

$2.3 \quad \mathrm{XR}-\mu \mathrm{CT}$ representation of sample $24-99 \ldots \ldots \ldots \ldots$

2.4 Garnet X-ray maps . . . . . . . . . . . . . . . . . . . . . . . . . . . 59

$2.5 \quad$ Garnet compositional line profiles $\ldots \ldots \ldots \ldots \ldots$

2.6 Radius-rate method schematic . . . . . . . . . . . . . . . . . . 61

$2.7 \quad$ Radius-rate results $\ldots \ldots \ldots \ldots \ldots$

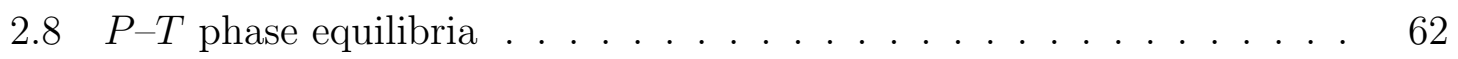

$2.9 \quad$ Schematic molar Gibbs energy-composition $(G-X)$ diagram $\ldots . .63$

2.10 Parallel and common tangent method compositions . . . . . . . 63

2.11 Theria_G model schematic . . . . . . . . . . . . . . . . . . 65

2.12 Phase equilibria and garnet population constraints for fractionating bulk chemistry along $P-T$ path $\ldots \ldots \ldots$. . . . . . . . 67

2.13 Simulated garnet compositional profiles . . . . . . . . . . . . 67 
2.14 Effective shape radius . . . . . . . . . . . . . . . . . . . . . 69

2.15 Schematic garnet profiles . . . . . . . . . . . . . . 69

3.1 CSD and major element chemistries and simulations for 24-99 . . . . 108

$3.2 \quad \mathrm{X}-\mathrm{Y} \mathrm{HR}-\mu$-CT showing spatial distribution of largest garnets . . . . . 109

3.3 LA-ICP-MS raster maps for crystal CT252 . . . . . . . . . . . . . . 109

3.4 LA-ICP-MS raster maps for crystal CT262 $\ldots \ldots \ldots \ldots$

3.5 LA-ICP-MS raster maps for crystal CT253 . . . . . . . . . . . . . . 112

3.6 LA-ICP-MS raster maps for crystal CT176 . . . . . . . . . . . . . . 113

3.7 LA-ICP-MS raster maps for crystal CT241 . . . . . . . . . . . . . . 114

3.8 LA-ICP-MS raster maps for crystal CT246 . . . . . . . . . . . . 115

3.9 Schematic HREE+Y zoning illustration and REE line profiles . . . . 116

3.10 FE-EPMA Y maps . . . . . . . . . . . . . . . . . . . 118

$3.11 \mathrm{Lu}$ and Ho compositional profiles plotted by distance from rim . . . . 119

3.12 Location and chemical compositions at garnet annuli . . . . . . . . . 120

3.13 Relative distribution of chromium and included phases in garnet . . . 121

3.14 Volume-corrected core-rim trace element profiles . . . . . . . . . . . . 122

$4.1 \quad$ Map of the Sikkim Himalaya and sample locations . . . . . . . . . . . 183

$4.2 \mathrm{XR}-\mu \mathrm{CT}$ reconstructions $\ldots \ldots \ldots \ldots$

4.3 Petrography: MAN1, 24-99, MS7A, MAN22 . . . . . . . . . . . . 185

4.4 Compositional line profiles through garnet: MAN1, 24-99, MS7A, MAN22186

4.5 Petrography: SIM3 and Type A garnet . . . . . . . . . . . . . . . . . 187

4.6 Compositional zoning in garnet: SIM3 . . . . . . . . . . . . . . . . . 189

4.7 Compositional zoning through garnet: SIM3 . . . . . . . . . . . . . . 190

4.8 Petrography: SIM3 and Type B . . . . . . . . . . . . . . . . . . . 191

4.9 AFM projection $\ldots \ldots \ldots \ldots$. . . . . . . . . . . . . . . . . 192 
4.10 EBSD SIM3 Map $1 \ldots \ldots$

4.11 EBSD SIM3 Map $2 \ldots \ldots \ldots$

4.12 Epitaxy in SIM3 Map 2 . . . . . . . . . . . . . . . . . . . . 195

4.13 Phase equilibria: MAN1, MS7A, MAN22 … . . . . . . . . 196

4.14 Garnet crystallisation: MAN1, MS7A . . . . . . . . . . . . 197

4.15 Phase equilibria: SIM3 $\ldots \ldots \ldots \ldots$

4.16 Combined $P-T-t-D$ results $\ldots \ldots \ldots \ldots$

4.17 Effect of $\mathrm{Na}_{2} \mathrm{O}$ on garnet stability $\ldots \ldots \ldots \ldots \ldots \ldots$

$5.1 \quad$ FE-EPMA grain boundary maps $\ldots \ldots \ldots \ldots \ldots$ 


\section{List of Tables}

2.1 Bulk composition of sample $24-99 \ldots \ldots \ldots \ldots \ldots$

$3.1 \quad$ Strain rate calculations for garnet in sample 24-99 . . . . . . . . . . . 107

$3.2 \quad$ Matrix phase EPMA and and LA-ICP-MS spot analyses . . . . . . . 107

4.1 Garnet textural parameters from the northern IMS . . . . . . . . . . 178

4.2 Bulk chemical compositions . . . . . . . . . . . . . . . . . . 178

4.3 Modal proportions . . . . . . . . . . . . . . . . . . . . . . 179

$4.4 \quad$ EPMA mineral chemistry: MAN1, 24-99, MS7A, MAN22 . . . . . . . 180

4.5 EPMA mineral chemistry: SIM3 . . . . . . . . . . . . . . . . . . . . 181

4.6 EBSD epitaxy measurements . . . . . . . . . . . . . . . . . . . . 182 


\section{Chapter 1}

\section{Introduction}

\subsection{General background}

Mineral crystallisation in the solid-state is the sum of a series of processes that facilitate the nucleation of new phases, the dissolution of source material, the transfer of components to and from reacting sites, and growth by further precipitation on pre-existing surfaces (e.g., Kretz, 1974; Casey \& Ludwig, 1996, Putnis, 2002, Baumgartner et al., 2013; Putnis, 2014). Of metamorphic minerals, garnet is perhaps the most widely studied, as its microstructural relationships with other phases and chemical zonation often document an unparalleled record of metamorphic conditions, processes, timings, and rates (e.g., Kretz, 1973; Spear \& Selverstone, 1983, Hickmott et al., 1987; Carlson, 1989; Bell \& Johnson, 1989, Burton \& O’Nions, 1991, Chu \& Ague, 2015b). In particular, its chemical sensitivity to changing extrinsic conditions combined with slow intracrystalline divalent cation diffusivites at low-medium grade conditions make garnet ideally suited to thermobarometric evaluation to determine the metamorphic history over which it crystallised (e.g., Spear et al., 1984; Vance \& Mahar, 1998; Gaidies et al., 2006). Much of our understanding of prograde crystallisation is therefore deeply rooted in studies of garnet porphyroblasts, which most commonly utilise the equilibrium model of rock metamorphism. 
However, because assumptions of equilibrium preclude consideration of rate-controlling kinetic barriers or the departures from equilibrium required for both mineral nucleation and growth, they are fundamentally at odds with processes in natural samples. Studies utilising equilibrium thermodynamics have therefore evolved in relative isolation from those concerned with the specifics of the kinetics of mineral crystallisation (e.g., Kretz, 1974; Fisher, 1978, Lasaga \& Kirkpatrick, 1981; Walther \& Wood, 1984 Loomis et al., 1985, Carlson, 1989), which are fundamentally controlled by disequilibrium across the rock volume. There is increasing chemical and textural evidence that impediments to a reaction and limited rates of mass transfer may prevent rocks from approaching chemical equilibrium during prograde $P-T$ changes (e.g., Manning et al., 1993 Waters \& Lovegrove, 2002, Pattison \& Tinkham, 2009, Carlson et al., 2015 and references therein). In such cases, application of an equilibrium approach may lead to spurious interpretations or oversimplifications of metamorphic histories (e.g., Austrheim, 1987).

Given the increasing recognition of the importance of kinetic controls on metamorphism, there have been numerous attempts to integrate the approaches to better constrain our understanding of metamorphic crystallisation along $P-T$ paths in complex multicomponent rock systems (e.g., Gaidies et al., 2011; Pattison et al., 2011; Kelly et al., 2013). With an increased awareness of the more nuanced aspects of metamorphic crystallisation, greater uncertainty than ever exists as to whether kinetic inhibition of mineral reactions and the development of significant disequilibrium is widespread throughout a range of metamorphic rock types.

The availability of major elements is generally thought to be rate-controlling during most solid-state mineral crystallisation reactions (e.g., Carmichael, 1969), and major elements are therefore often used in order to determine the extent to which thermodynamic equilibrium was approached during metamorphic crystallisation pro- 
cesses (e.g., Gaidies et al., 2011; Pattison et al., 2011; Kelly et al., 2013). Moreover, major element diffusion modelling has proven valuable in constraining the rates of metamorphic processes associated with garnet crystallisation (e.g., Lasaga, 1983 , Ague \& Baxter, 2007; Viete et al., 2011). While significant effort has been made to understand the nature and efficacy of transport of major elements during porphyroblast crystallisation (e.g., Fisher, 1973, Carlson, 1989; Spear \& Daniel, 2001; Carlson \& Gordon, 2004, Konrad-Schmolke et al., 2005), an equivalent understanding of the distribution of trace elements does not exist, in part a consequence of the variability with which the zoning of these elements manifests in porphyroblasts (e.g., Hickmott \& Shimizu, 1990; Pyle \& Spear, 1999; Yang et al., 1999; Moore et al., 2013), and partly because of only relatively recent analytical advances that allow comprehensive characterisation of two-dimensional trace element distributions in mineral phases (c.f. Raimondo et al. 2017). Owing to their relatively slow diffusivities compared to major elements (e.g., Cherniak, 2005; Carlson, 2012), the distribution of trace elements in porphyroblastic phases may yield nuanced insight into subtle controls on metamorphic crystallisation that cannot be gleaned from major element analysis alone.

Microstructures are not generally considered during direct application of the equilibrium model, but may vary relatively systematically with changes in $P-T$ across metamorphic field gradients (e.g., Spry, 1969, Cashman \& Ferry, 1988). Moreover the extent and degree to which a chemical system is equilibrated during a metamorphic mineral reaction is likely to impact the size, spatial distribution, morphology and/or chemical compositions of product porphyroblasts (e.g., Kretz, 1969, Hickmott \& Shimizu, 1990; Chernoff \& Carlson, 1997). As a result, methods that integrate comprehensive microstructural and chemical datasets with models of metamorphic crystallisation may provide insights into the mechanisms of the rate-limiting processes 
that control metamorphism, and shed light on the potentially systematic development of porphyroblastic microstructure (e.g., Kretz, 1966, Kretz, 1969, 1974; Thompson, 1986; Cashman \& Ferry, 1988; Chernoff \& Carlson, 1997; Daniel \& Spear, 1999 Konrad-Schmolke et al., 2005). Given garnet's high density and X-ray attenuation at a range of energies, it is well-suited to quantitative textural analysis using X-ray $\mu$-computed tomography (XR $\mu$-CT), making it possible to accumulate high volumes of data pertaining to the size, shape, and spatial distributions of garnet populations (e.g., Ketcham \& Carlson, 2001, Denison \& Carlson, 1997).

This thesis therefore uses an approach that integrates three-dimensional (3D) microstructural constraints, high-resolution chemical analyses and numerical and thermodynamic equilibrium approaches in order to shed light on the conditions of and processes controlling garnet nucleation and growth in rocks from the Sikkim Himalaya, in northeastern India. As a consequence of its relatively well-constrained geological history and well-developed inverted Barrovian sequence (e.g., Mohan et al., 1989, Dasgupta et al., 2004; Mottram et al., 2014a; Gaidies et al., 2015), Sikkim provides an unparalleled natural laboratory in which to contribute to the discussion surrounding fundamental rock-forming processes that operate during regional metamorphism. In particular, this work investigates the extent and cause of chemical and microstructural heterogeneities in low-medium grade metapelites, lithologies that are increasingly thought to contain a record of persistent disequilibrium during prograde metamorphism (e.g., Wilbur \& Ague, 2006, Pattison et al., 2011; Wolfe \& Spear, 2018). Moreover, given extensive and unparalleled geochemical, microstructural and thermobarometric constraints, this thesis presents some of the most comprehensively characterised garnet populations ever presented, allowing uniquely detailed insight into garnet crystallisation during Barrovian metamorphism. 


\subsection{Metamorphic crystallisation}

\subsubsection{Nucleation}

Nucleation is the initial stage of the formation of a new phase that serves to lower the free energy of the system, and thus increases its stability. In metamorphic petrology, the widespread application of equilibrium thermodynamics means that the process of nucleation is oversimplified, with something akin to instantaneous barrier-free nucleation of a phase that serves to lower the Gibbs energy, $G$, of the system. However, in real systems, a departure from equilibrium (commonly referred to as overstep) is required to overcome the energy barrier associated with chemical and structural differences between the precursor and the newly forming nuclei.

Classical nucleation theory (CNT) is perhaps the best developed model of nucleation, and allows us to describe the energy barrier to nucleation during a discontinuous phase transformation in a homogeneous system (Volmer \& Weber, 1926). As outlined by Gaidies (2017), CNT approximates an inherently interface-controlled system, as diffusion in the matrix is infinitely fast, and thus not considered. Clusters of molecules grow and shrink by addition and loss of individual molecules (e.g., Turnbull \& Fisher, 1949), and for nucleation of a cluster of $n$ molecules with an isotropic surface across $i$ regions, $A_{i}$, each with an interfacial energy of $\sigma$, the Gibbs energy of formation of the cluster $\left(\Delta G_{n}\right)$ is a function of both the volume of the cluster, and its surface:

$$
\Delta G_{n}=n \Delta G_{V}+\sum_{i} A_{i} \sigma_{i}
$$

where $\Delta G_{V}$ is the difference in bulk Gibbs energy between the reacting system and nucleating phase, per product molecule.

In Eq. (1.1) the first term on the right hand side describes the difference in bulk 


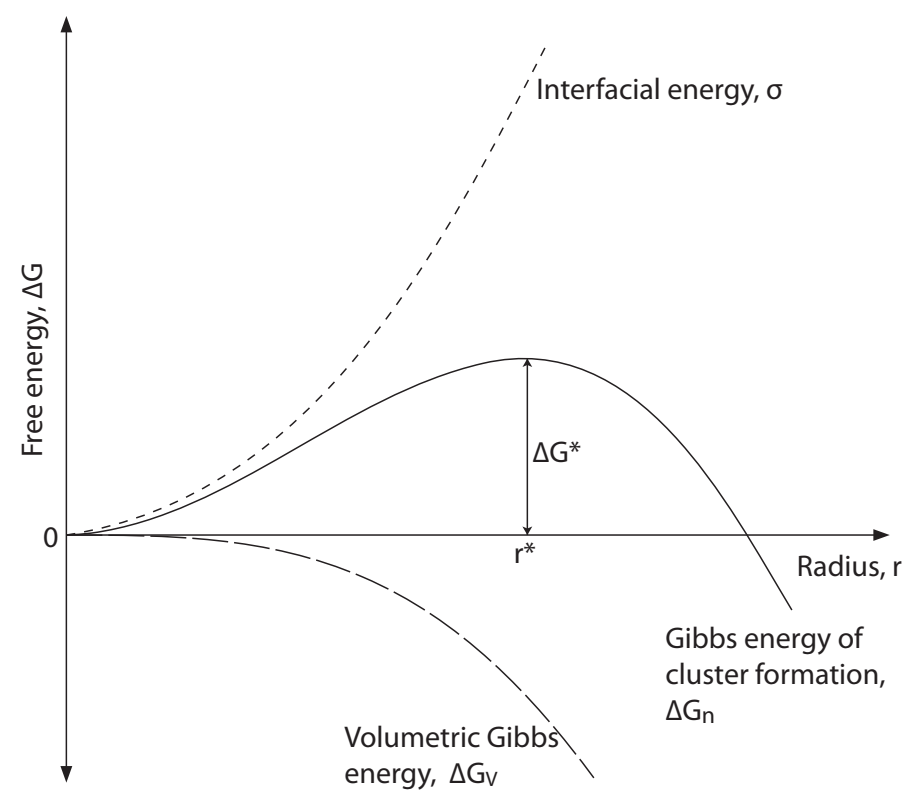

Figure 1.1: Gibbs energy of cluster formation, $\Delta G_{n}$ as a function of radius. Shown is the interfacial energy contribution to nucleation (short dashed line), and the bulk Gibbs energy term (long dashed line) which is always negative and serves as a driving force. The critical energy barrier to nucleation, $\Delta G *$ occurs at a cluster radius $r *$.

free energy per unit volume between the phase that is nucleating and the phase that nucleation occurs in, and is always negative at conditions when the product-forming reaction is thermodynamically favoured. Conversely, the second term on the right hand side is always positive, as it is a surface term that is proportional to the extent and energy of the surface between the nucleus and its surroundings. This surface term acts as a barrier to nucleation. Figure 1.1 illustrates the relative contribution of each of these terms to the total bulk Gibbs energy. Nucleation does not occur until the maximum energy barrier to nucleation, $\Delta G^{*}$, is overcome, which occurs when a cluster has a critical radius, $r^{*}$ (Fig. 1.1). While not considered in Eq. (1.1), lattice misfits between the precursor and the nucleating phase are also of critical importance in natural samples, as they involve elastic strain energy. This energy adds an additional component to the barrier to nucleation, $\Delta G_{E}$, so that $\Delta G^{*}$ is increased (Lee et al. 1977). 
CNT is an inherently surface-free model, with nucleation in a chemically and structurally homogeneous matrix. However in a solid-state polycrystalline material, heterogeneous nucleation better approximates reality, as phases nucleate on pre-existing surfaces. This is kinetically favourable, because the energy of a pre-existing surface will contribute to the energy required to overcome the energy barrier to nucleation, effectively reducing $\Delta G^{*}$. Heterogeneous nucleation describes the contact between a cluster and a surface by some angle, $\theta$. With decreasing $\theta$ from $180^{\circ}$ (effectively a spherical cluster, equivalent to homogeneous nucleation) to $0^{\circ}$ (total wetting of the surface), the Gibbs energy of the cluster on a surface, $\Delta G_{\text {surf }}$, decreases relative to $\Delta G^{*}$. With some exceptions (e.g., a phase nucleating on a surface from a fluid), solid-state nucleation occurs on defects (grain edges, grain boundaries and corners), bound by either the same phase or two phases, and different characteristic surfaces should influence nucleation to differing degrees, yielding a range of highly-favourable to less-favourable nucleation sites within a rock volume.

Deformation related defects (i.e., dislocations) also aid nucleation, as the elastic strain energy within a dislocation is released upon nucleation and may counteract the elastic strain energy associated with lattice misfits described above. It is not well understood how nucleation and dislocations facilitates nucleation, but may be of particular relevance in highly deformed metamorphic rocks.

\subsubsection{Growth}

Growth is the advance of a mineral interface formed by nucleation into the surrounding medium by way of addition of atoms. Like nucleation, the process is associated with energy barriers that must be overcome by sufficiently high driving forces. In contrast with nucleation, which is dominated by the energy required to form the interface, barriers and impedances to growth are mainly associated with the rate of long-range 
transport to and from reacting surfaces, and the attachment and detachment of atoms at the interface.

Generally, two end-members of porphyroblastic mineral growth are identified: (1) interface-controlled growth and (2) transport-controlled growth. In the case of interface-controlled growth, the relative rates of long-range transport of components to and from reacting sites is rapid relative to dissolution or precipitation at the moving interface (e.g., Ridley \& Thompson, 1986, Carlson, 1989). In this case, rapid component transport imposes a spatially uniform intergranular medium composition, such that chemical disequilibrium develops only in the direct vicinity of the interface. Conversely, in the case of transport-controlled growth, the transport of components through the rock (generally by advection or diffusion) is slow relative to processes at the propagating interface. While advection and diffusion may act in parallel, advective transport in a mobile fluid phase is most relevant in open-systems (for example, in hydrothermal systems or where there is significant strain localisation that channelises fluids) whereas solid-state diffusional transport (by inter and intragranular diffusion) dominates in approximately closed systems with low fluid-to-rock ratios. In these cases, local equilibrium is only expected at the interface, and chemical potential gradients are expected to develop across the rock matrix as the porphyroblast grows. These gradients are a function of element diffusivity, and will thus vary between components (Fig. 1.2), with some elements equilibrated and others not.

In real and variable metamorphic systems, it is unlikely that interface- and diffusioncontrolled growth are mutually exclusive in a given rock volume, and rate-limiting mechanisms may exist on a spectrum. Rate-limiting mechanisms may also vary over the duration of metamorphism, according to the changing extrinsic conditions and rock reactivity. In addition, other rate-limiting mechanisms have been proposed (e.g., Fisher, 1978, Dohmen \& Chakraborty, 2003). Characteristic 3D spatial distributions 


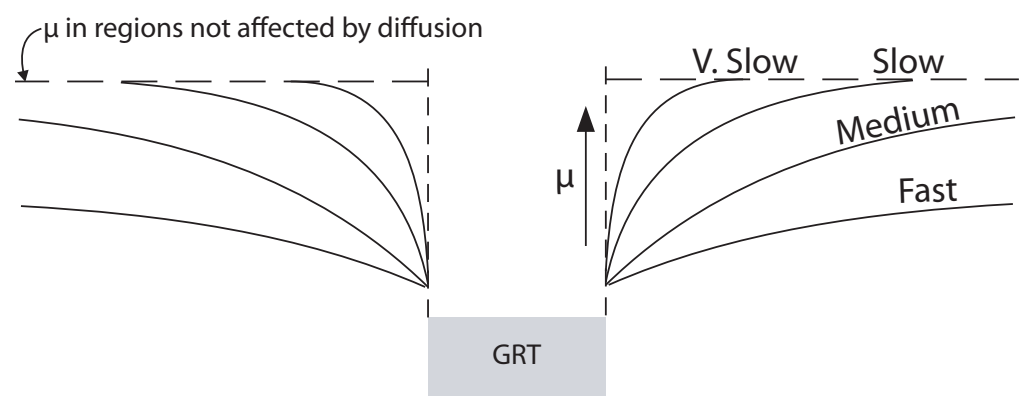

Figure 1.2: Schematic chemical-potential gradients around growing porphyroblasts for elements with different intergranular diffusivities. After Carlson (2002).

of mineral phases (i.e., clustering or ordering) may also be produced by end-member rate-limiting mechanisms (e.g., Carlson, 1989; Denison \& Carlson, 1997), which can be interrogated using statistical approaches (Hirsch, 2011). This approach may be of great value in contact metamorphic settings (e.g., Petley-Ragan et al., 2016) as deformation may be limited and the observed positioning of porphyroblasts may reflect their primary distribution. However, in regionally metamorphosed settings, pervasive deformation means that grains are unlikely to retain their original sites of nucleation and growth, such that statistical methods of interpreting porphyroblast distributions may produce spurious results.

In both end-member scenarios of porphyroblast crystallisation, relatively slow rates of intracrystalline diffusion may result in the development of chemical disequilibrium across porphyroblast volumes, potentially archiving a record of the mechanisms and relative rates of the atomic-scale processes of crystallisation. As a consequence of relatively slow intracrystalline major element diffusivities which can preserve a compositional record of crystallisation, garnet has traditionally been the primary mineral used in studies of this nature. Previous assessment has yielded a wide range of results (e.g., Carlson, 1989, Kretz, 1993; Denison \& Carlson, 1997, Chernoff \& Carlson, 1997 Spear \& Daniel, 1998; Daniel \& Spear, 1999), with little consensus as to the dominant rate-limiting mechanism for garnet porphyroblast crystallisation. 


\subsubsection{Diffusion}

Diffusion arises from the thermally-driven atomic scale net movement of atoms, ions, or particles, in minerals, glasses, melts, fluids and gases. Random walk of the diffusing component occurs between domains due to chemical potential differences, and will yield an overall flux when the chemical potential gradient is not uniform. Given sufficient time, diffusion usually serves to equilibrate the system in question until chemical equilibrium is attained.

The fundamentals of all diffusion theory lie in Fick's first and second laws (written here in terms of concentrations and in simple one-dimensional form to describe binary diffusion):

$$
J_{i}=-D_{i} \frac{\delta C_{i}}{\delta x} \quad(1.2) \quad \frac{\delta C_{i}}{\delta t}=D_{i} \frac{\delta^{2} C_{i}}{\delta x^{2}}
$$

These equations link the flux, $J$, and concentration, $C$, of species $i\left(J_{i}, C_{i}\right.$, respectively), to time $(t)$, distance $(x)$, and the diffusivity of component $i\left(D_{i}\right)$. Fick's first law (Eq. 1.1) shows that when a concentration gradient is large, the diffusive flux along that is also large; Fick's second law (Eq. 1.2) describes the evolution of a concentration profile over time across a given space. Sensu stricto, chemical fluxes occur in response to gradients in chemical potential $\left(\frac{d \mu}{d x}\right)$, which can differ from gradients in concentration (as used above) due to non-ideal thermodynamic interactions and to coupling between multiple interdiffusing species. Ganguly (2002) presents a thorough modification of Equations (1.1) and (1.2) for chemically complex, thermodynamically non-ideal mineral systems. Diffusion in minerals (e.g., garnet) is often multicomponent, due to the flux dependence of components not only on their own concentrations or chemical potential gradients, but also of those of all other diffusing components. For multicomponent diffusion, Fick's second law (Onsager, 1945) in an 
$n$ component system takes the form

$$
\frac{\delta C_{i}}{\delta t}=\frac{\delta}{\delta x} \sum_{j=1}^{n-1} D_{i j} \frac{\delta C_{j}}{\delta x}
$$

where $D_{i j}$ represents the interdiffusion coefficients of $n-1$ components, and can be calculated using the method described by Lasaga (1979). For given chemical conditions, $D_{i j}$ is sensitive to the diffusion coefficient of each component, $D_{i}$, which is described by the Arrhenius equation:

$$
D_{i}=D_{0} \exp \left[\frac{-E_{A}+P \Delta V}{R T}\right]
$$

where $T$ is temperature, $P$ is pressure, and $R$ is the universal gas constant. The pre-exponential factor $\left(D_{0}\right)$, the activation energy $\left(E_{A}\right)$, and the activation volume $(\Delta V)$ are all Arrhenius parameters that can be determined experimentally at prescribed conditions (e.g., tracer diffusion experiments and diffusion-couple experiments; Chakraborty \& Ganguly, 1992; Vielzeuf et al., 2007) or semi-empirically by forward modelling diffusion profiles (e.g., Carlson, 2006). For most diffusion data the temperature range is not broad enough for geological applications, so the Arrhenius relation works well within uncertainty to extrapolate $D$.

As can be seen in Eq. (1.5) diffusivity is strongly temperature dependent, and the Arrhenius equation plots $\log D_{i}$ versus $1 / T$ as a straight line with a negative slope, i.e., higher $T$ yields exponentially higher $D_{i}$. The relationship between diffusivity and $P$ is more complex and is related to the significant variation expected for $\Delta V$ over a wide range of pressures, often yielding a non-linear $\log D_{i}$ versus $P$. However, over a small pressure interval, $\log D_{i}$ is most often linear in $P$ : a positive $\Delta V$ will result in decreasing $D_{i}$ with increasing $P$, and a negative $\Delta V$ results in increasing $D_{i}$ with increasing $P$ (Zhang, 2010). 
As diffusion in a solid crystalline material is essentially the random walk of the species through the defects of a crystal structure, $D_{i}$ is often proportional to the defect density, whereby a high defect concentration increases the diffusivity. Point defects (e.g., vacancies) play the most important role in enhancing rates of diffusion, but extended defects (e.g., dislocations, domain boundaries, and grain boundaries) also produce fast diffusion pathways. When assessing chemical diffusion through a rock, we consider both isotropic and/or anisotropic volume diffusion through a crystal lattice (intracrystallline diffusion) and diffusion along 3D networks of extended defects that form grain and interphase boundaries (intercrystalline diffusion). As a general rule of thumb, grain boundary diffusion is several orders of magnitude faster than volume diffusion (e.g., Joesten, 1991; Farver \& Yund, 1992, Farver, 1994), and can facilitate mass transfer on much larger scale (not considering concurrent advection of material and diffusion in an advecting grain boundary fluid). Given also that deformation increases defect concentration, extensive deformation should increase diffusivities. Cohen (1970) describes two different states arising from the influence of deformation: (1) dislocation-assisted diffusion, when prior deformation has created dislocations, and (2) strain-enhanced diffusion, when dislocations form during diffusion.

In addition to dependence on $T, P$, and defect density, diffusivity is dependent on the phase in which volume diffusion occurs (e.g., see Watson \& Baxter, 2007) and can also be dependent on the $\mathrm{H}_{2} \mathrm{O}$ pressure or fugacity $\left(P_{\mathrm{H}_{2} \mathrm{O}}\right.$ or $\left.f_{\mathrm{H}_{2} \mathrm{O}}\right)$. For the case of cation diffusion in solids, slightly enhanced $D_{i}$ at high $P_{\mathrm{H}_{2} \mathrm{O}}$ or $f_{\mathrm{H}_{2} \mathrm{O}}$ is generally ascribed to the increase in vacancy concentration with increasing $\mathrm{H}^{+}$ and $f_{\mathrm{H}_{2} \mathrm{O}}$ (Costa \& Chakraborty, 2008; Fei et al., 2013). Furthermore, diffusion in some minerals (including garnet, rutile, pyroxene) has been shown to be dependent on oxygen fugacity, $f_{\mathrm{O} 2}$ in several different ways (Sasaki et al., 1985; Chakraborty \& Ganguly, 1992; Cherniak \& Dimanov, 2010). Increasing the valence state of an 
ion serves to strengthen the bonds between the ion and the rest of the structure, decreasing the diffusivity. Conversely, oxidation of $\mathrm{Fe}^{2+}$ to $\mathrm{Fe}^{3+}$, for example, induces site vacancy defects, which serve to increase the effective diffusivites.

\subsection{The Sikkim Himalaya}

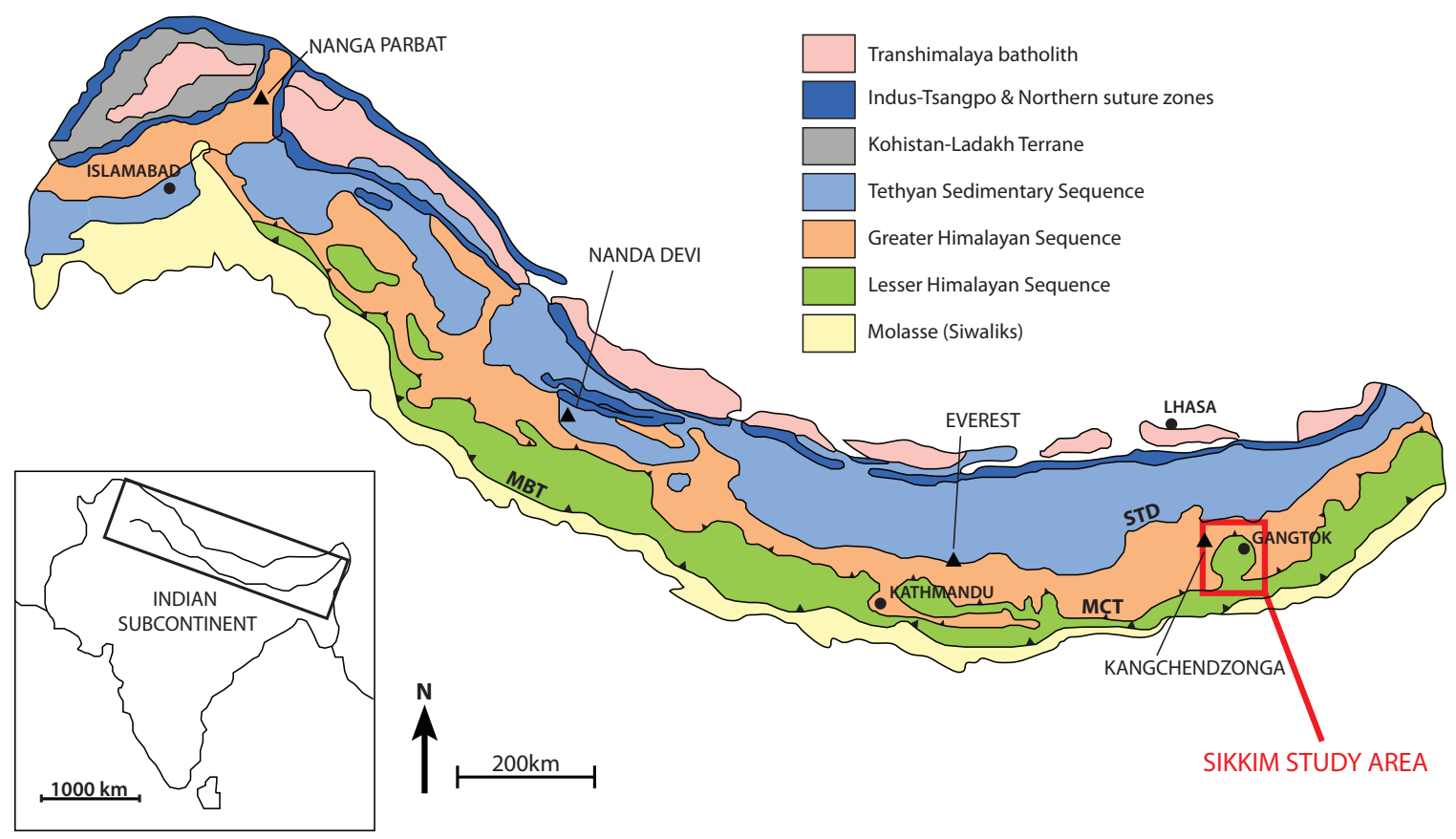

Figure 1.3: Overall tectonic map of the Himalayan orogen.

Some of the earliest geological studies of the Himalaya are based on the Darjeeling Hills and Sikkim region (e.g., Mallett, 1875, Bose, 1891; Gansser, 1938). Collectively known as the Sikkim Himalaya, the region is situated in north-east India, sandwiched between Nepal and Bhutan, near the eastern syntaxis of the Himalayan orogen (Fig. 1.3). The large-scale structure of Sikkim closely resembles that of other Himalayan regions, resulting from ongoing northward motion of the Indian plate and associated thrust-accommodated crustal shortening since 55-50 Ma (de Sigoyer et al., 2000). In Sikkim (Fig. 1.4), there is exposure of a full structural and stratigraphic transect 

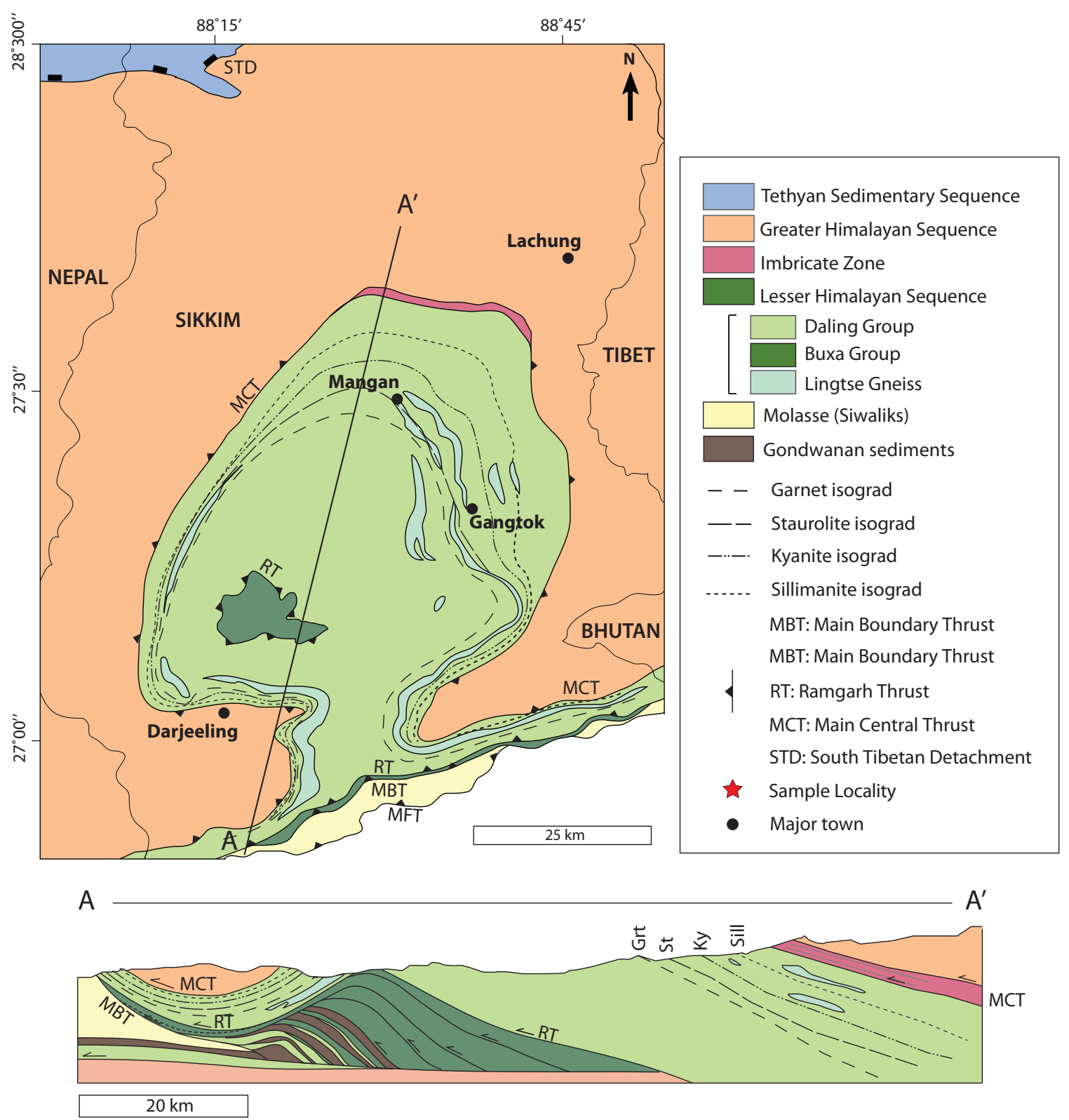

Figure 1.4: (a) Geological map of the Sikkim Himalaya. (b) Cross section through A-A'. Modified after Chakraborty et al. (2016) and Mottram et al. (2014b).

through the orogen (e.g., Mohan et al., 1989; Dasgupta et al., 2004). Directly north of the southernmost unmetamorphosed Miocene-Pliocene Siwalik shelf sediments is the low-medium grade Lesser Himalayan Sequence (LHS), which is bounded by the orogen-parallel Main Boundary Thrust (MBT) and Main Central Thrust (MCT) zone 
to the south and north, respectively (Goswami, 2005). North of the MCT zone, the high grade Greater Himalayan Sequence (GHS) and associated batholiths form the High Himalaya. The GHS is a package of metapelites, orthogneisses, migmatites and leucogranites derived from Proterozoic-early Paleozoic sediments that were intruded by Neoproterozoic and Cambrian granites. Deformation, metamorphism and partial melting of the GHS occurred during Himalayan orogenesis.

The LHS of the Sikkim Himalaya can be divided into two primary clastic metasedimentary units: the Paleoproterozoic Daling Group and Permian-Carboniferous Buxa and Gondwana group sediments, both associated with large bodies of Paleoproterozoic orthogneiss, known locally as the Lingtse Gneiss (Fig. 1.4. e.g., Paul et al., 1982, Mottram et al., 2014a). The Ramgarh Thrust (RT) separates the underlying Buxa Group and Gondwana sediments from the Daling Group Mukul, 2000, Mukul et al., 2007), and the latter is the most abundant rock type of the LHS in the Sikkim Himalaya. Rocks of the Daling Group form a domal structure (the Teesta Dome) in the south-central part of Sikkim, with relative structural dips towards each respective flank. The Buxa Group forms a major duplex structure below the RT (the Lesser Himalayan Duplex), which is exposed in the Rangit Window (Bhattacharyya \& Mitra 2009: Bhattacharyya, 2010). Movement along the RT and formation of the Lesser Himalayan Duplex is thought to have accommodated up to $95 \%$ of shortening in the lesser Himalayan rocks (Mitra et al., 2010). Also contained within the Daling Group are several sheared and lenticular bodies of Precambrian Lingtse Gneiss at numerous structural levels that are subparallel to the structure of the Teesta Dome; slices of this gneiss are interpreted as basement (Sinha-Roy, 1982).

Moving from the core of the Daling Group in the Teesta Dome towards the GHS to the west, north and east, chlorite-biotite phyllites give way to a well-documented Barrovian metamorphic sequence (Fig. 1.4), where progressively higher grade rocks 
occur at higher structural levels (e.g., Mohan et al., 1989, Dasgupta et al., 2004). This is known as an inverted metamorphic sequence, and the locality in Sikkim was one of the first identified regions exposing such a geological feature (Bose, 1891). In some studies, rocks beyond the kyanite zone are classified as a separate group, the Paro Group (e.g., Mitra et al., 2010). However, there is little evidence for this group (c.f., Chakraborty et al., 2016), and so it is not distinguished from the Daling Group in this work. Towards the shallow levels of the Daling Group, the MCT is exposed as a wide reentrant, juxtaposing rocks of the LHS onto the GHS (with the latter sometimes known as the Darjeeling Group in Sikkim). The precise location and nature of the high-strain 'MCT zone' is subject to significant debate (e.g., Bose 1891; Catlos et al., 2004; Martin et al., 2005; Mottram et al., 2014a; Bhattacharyya \& Mitra, 2009; Gupta et al., 2010; Bhattacharyya, 2010, Bhattacharyya \& Mitra, 2011), and remains an outstanding tectonometamorphic uncertainty in Sikkim.

\subsubsection{Sample collection}

Fieldwork for this study was carried out in the Sikkim Himalaya over two field seasons in March 2012 by Dr. Fred Gaidies, and March 2015 by Freya George and Dr. Fred Gaidies. As this study focuses only upon metapelitic samples within the Barrovian sequence of the LHS in Sikkim, fieldwork was conducted to collect samples of this nature for the petrographic and geochemical analysis that form the basis of this thesis. Samples were largely collected along road transects, due to the heavily vegetated nature of the foothills of the Sikkim Himalaya. Localities visited and collection sites of the sample suite are displayed in Figure 1.5 . Chapters 2 and 4 of this thesis also present specific sample locations of relevance to Chapters 2 and 3, and Chapter 4 , respectively. 

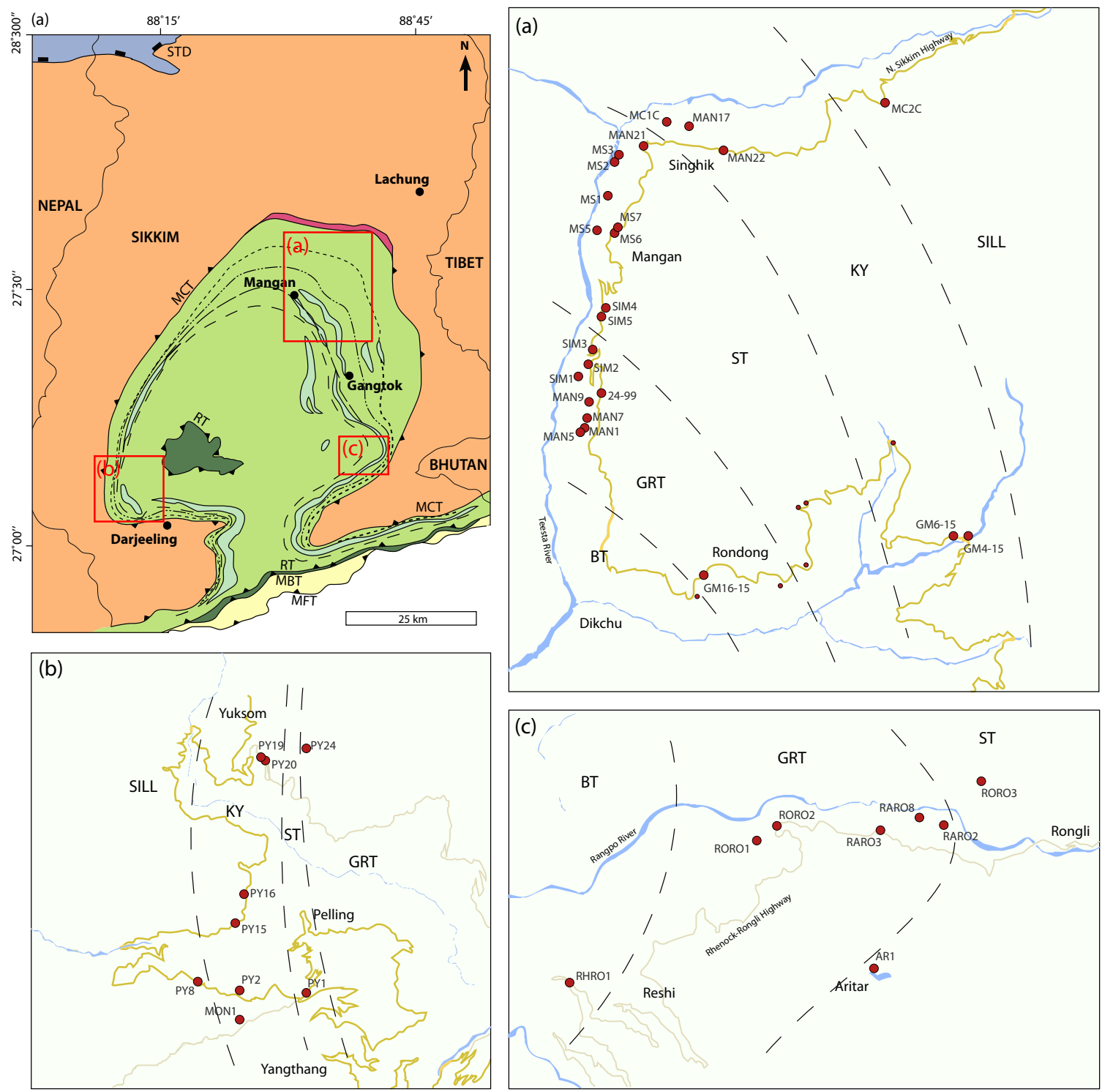

Figure 1.5: Locations of samples collected during 2012 and 2015 field seasons. 


\subsection{Thesis objectives}

This study aims to present a detailed petrological, geochemical, textural and thermobarometric study of the Daling Group of the LHS in the Darjeeling-Sikkim region of the eastern Himalaya, in order to elucidate the controls on the mechanisms and rates of metamorphic mineral crystallisation in these rocks. Comprehensive chemical characterisation of garnet was conducted in order to investigate the extent of chemical equilibration in the rock volume, and to assess the relative influence disequilibrium and rate-limiting kinetics may have had on garnet crystallisation. Microstructural and thermobarometric constraints are also used to evaluate the extent of metamorphic continuity from low-to-high grade.

This work principally utilses the study of garnet within these metapelitic schists not in terms of single porphyroblastic crystals, but with consideration of the whole population of crystals developed during protracted crystallisation. Specifically, the approach uses a combination of analytical techniques (high-resolution $\mu$-computed tomography, electron probe microanalysis, laser ablation-inductively coupled plasmamass spectrometry, electron backscatter diffraction) and numerical and thermobarometric methods (phase equilibria modelling, diffusion modelling).

\subsection{Thesis structure}

This thesis is presented as three research chapters, in addition to an introduction and conclusion, that have been written to address the objectives outlined above as an integrated thesis. Each chapter is structured as a standalone manuscript with an abstract, introduction, methods and results section, discussion, conclusion and supplementary data that form the appendices of this thesis. As a result of this formatting choice, there is some repetition. After the introduction, Figures and Tables 
are presented at the end of each chapter.

Chapter 2 is a detailed major-element study of a population of garnets from the LHS of the Sikkim Himalaya that assesses the extent of rock-wide major element equilibration. Chemical zoning in garnets representative of the population is assessed, and thermobarometric approaches coupled with diffusion modelling constrain the average rate of metamorphism. Chapter 3 assesses the trace element distribution in the same population as presented in Chapter 2. Trace elements are used to better constrain subtle aspects of the garnet crystallisation history, characterise partial disequilibrium in the rock volume, and to contribute to our understanding of strain records in pervasively deformed rocks. Chapter 4 utilises samples from a range of Barrovian zones in Sikkim to constrain the microstructural, structural and thermobarometric continuity of the IMS in Sikkim. Microstructural constraints help to develop empirical constraints on the matrix-dependent nucleation and growth of garnet populations. Chapter 5 follows the research chapters and summarises key conclusions and implications, and suggests directions for future research. 


\section{Chapter 2}

\section{Major element zonation of a garnet population: rates and mechanisms of crystallisation}

\section{$2.1 \quad$ Introduction}

Many techniques used in metamorphic petrology rely on the application of equilibrium thermodynamics in order to elucidate the complex history of metamorphic crystallisation (e.g., Spear et al., 1984, Vance \& Mahar, 1998; Cottle et al., 2011, Dragovic et al., 2012, Lanari et al., 2013; Konrad-Schmolke \& Halama, 2014). The equilibrium model of metamorphism necessitates that over the duration of a reaction, near-equilibrium conditions should be maintained, with the implication that kinetic barriers to crystallisation processes are insignificant. However, it is well understood that fundamental processes such as nucleation, crystal growth and chemical diffusion are driven by a departure from equilibrium, such that the equilibrium model may be limited when applied to metamorphic reactions associated with significant energy barriers (e.g., Fisher, 1973; Carlson, 2002, Skora et al., 2006; Pattison et al., 2011). Consequently kinetic impedances to chemical equilibration during metamorphism are increasingly being investigated, with particular focus on quantitatively constraining the magnitude of the departure from equilibrium with respect to temperature, pres- 
sure or Gibbs energy (Waters \& Lovegrove, 2002, Pattison \& Tinkham, 2009, Pattison et al., 2011; Gaidies et al., 2011; Spear et al., 2014; Carlson et al., 2015).

The spatial extent and degree to which a system is chemically equilibrated during a metamorphic mineral reaction is likely to impact the size distribution and chemical composition of product porphyroblasts (e.g., Chernoff \& Carlson, 1997, Hirsch et al., 2003). As a result, methods that integrate microstructural and chemical datasets with quantitative models of metamorphic crystallisation may provide insights into the mechanisms and rate-limiting processes that control metamorphism (e.g., Kretz, 1969, 1974; Thompson, 1986; Cashman \& Ferry, 1988b; Chernoff \& Carlson, 1997; Daniel \& Spear, 1998; Daniel \& Spear, 1999; Konrad-Schmolke et al., 2005; Kelly et al. 2013).

\subsubsection{Crystallisation processes}

The crystallisation of porphyroblasts is commonly considered to be a two-step process which includes both nucleation as the formation of the interface between product and matrix, and growth as the attachment of molecules to this product surface (e.g., Teng, 2013). This process is reliant on several discrete atomic-scale processes, including the dissolution of reactants, the attachment and detachment of atoms at the advancing interface between the crystallising product and its surrounding matrix, and the diffusional transport of components between the reactant and product sites (e.g., Casey \& Ludwig, 1996, Putnis, 2002; Baumgartner et al., 2013; Putnis, 2014). While nucleation and growth are thought to be controlled by different physiochemical parameters, each of these processes may be considered a potentially rate-limiting step governing the overall rate of metamorphic reaction (e.g., Walther \& Wood, 1984, Kretz, 1966 , Carlson, 2012).

Two end-member scenarios are commonly used to describe the kinetics of por- 
phyroblast nucleation and growth: diffusion- and interface-controlled crystallisation (e.g., Kretz, 1974, Lasaga, 1981, 1986, Carlson, 1989, Carlson \& Denison, 1992, Daniel \& Spear, 1998), although other controlling mechanisms have been postulated (e.g., Dohmen \& Chakraborty, 2003). In the case of diffusion-controlled crystallisation, there are sluggish rates of long-range diffusion of components between reacting sites relative to the rates of their attachment and detachment at the porphyroblast/matrix interfaces. Assuming nucleation in an initially thermodynamically homogeneous precursor assemblage, relatively slow long-range diffusion results in appreciable chemical potential gradients that develop and widen in the vicinity of crystallising grains (Fisher, 1978). As described by Carlson (1989), within these depleted zones the probability of continued nucleation of the product phase is reduced, and subsequent rates of mineral growth and the composition of the advancing crystal rims are controlled by the rate of diffusion across the matrix.

Conversely, interface-controlled crystallisation is assumed to take place if interfacial processes are significantly slower than long-range diffusion (Kretz, 1974). In this case, chemical disequilibrium may only be expected to develop across the interfaces between product and matrix phases. Relatively fast-diffusing components during interface-controlled crystallisation maintain a thermodynamically homogeneous and chemically equilibrated matrix so that the composition of all product crystal rims will be the same at any given time.

In both end-member scenarios of porphyroblast crystallisation, relatively slow rates of intracrystalline diffusion may result in the development of chemical disequilibrium across each porphyroblast volume, potentially archiving a record of both the mechanisms and relative rates of the atomic-scale processes of crystallisation. The investigation of metamorphic garnet, in particular, is ideally suited to such nuanced assessment because slow rates of intracrystalline diffusion within garnet commonly 
preserves a compositional record of its metamorphic crystallisation, which may in turn be interpreted with respect to the pressure-temperature-time $(P-T-t)$ evolution of the rock (e.g., Spear et al., 1984, Vance \& Mahar, 1998; Gaidies et al., 2006, Dragovic et al., 2012).

There is extensive literature on the use of populations of garnet porphyroblasts in order to constrain metamorphic crystallisation histories. Extraction of numerical growth rate laws from compositional zoning contained within garnet was pioneered by Kretz (1974), who derived general rate laws for interface- and diffusion-controlled growth. Major element garnet zoning profiles documented from two specimens by Finlay \& Kerr (1979) yielded a model of progressive garnet nucleation, interfacecontrolled rate laws, and the use of core $\mathrm{MnO}$ content as a timeline for nucleation. Subsequent work on additional samples indicated crystal-specific growth rates based on the comparison of garnet core $\mathrm{MnO}$ contents and crystal sizes, such that a general rate law could not be used to describe growth of the entire garnet population Finlay \& Kerr, 1987). Work on a similar dataset obtained by Anderson \& Olimpio (1977) led to the suggestion that significant thermal overstep of the equilibrium garnetforming reaction was required for garnet nucleation. More recently, the coupling of garnet compositional profiles and quartz-in-garnet (QuiG) barometry has resulted in the suggestion that overstep of the garnet-in isograd during the incipient stages of nucleation may commonly be significant (Spear et al., 2014; Castro \& Spear, 2016).

While the inferences drawn from such datasets have proven diverse, the collection of large datasets of compositional information continues to be key to understanding the mechanisms and processes that control porphyroblast growth. In this contribution, we characterise a population of garnet porphyroblasts within a garnet-grade metapelite from the Lesser Himalayan Sequence (LHS) in the Sikkim Himalaya. This region is known to contain a record of recent orogenesis (e.g., Mohan et al., 1989; 
Dasgupta et al. 2004), and therefore serves as an ideal natural laboratory in which to investigate the primary controls on porphyroblast crystallisation.

This chapter therefore uses an extensive chemical and microstructural dataset in order to test the applicability of the assumption of chemical equilibrium associated with garnet crystallisation in the studied sample, using both equilibrium and disequilibrium numerical models of garnet nucleation and growth. This approach provides constraints on the mechanisms that controlled garnet crystallisation, and derive estimates for the rate of metamorphic garnet growth associated with Barrovian metamorphism in Sikkim.

\subsection{Geological setting}

The Sikkim Himalaya is situated in north-east India, near the eastern syntaxis of the Himalayan orogen (Fig. 2.1). The large-scale structure of Sikkim closely resembles that of other Himalayan regions, resulting from the continued northward motion of the Indian plate and associated thrust-accommodated crustal shortening since 55$50 \mathrm{Ma}$ (de Sigoyer et al. 2000). The LHS is bounded by the orogen-parallel Main Boundary Thrust (MBT) and Main Central Thrust Zone (MCTZ) to the south and north, respectively. In Sikkim, the Paleoproterozoic Daling Group of the LHS contains a well-documented $\sim 15 \mathrm{~km}$-wide inverted Barrovian metamorphic sequence, with increasing grade towards the MCTZ (e.g., Paul et al., 1982; Mohan et al., 1989; Dasgupta et al., 2009; Mottram et al., 2014a).

Previously determined conditions of peak metamorphism in the inverted Barrovian sequence increase from c. $480-530{ }^{\circ} \mathrm{C}$ and $4.8-5.0 \mathrm{kbar}$ at the base of the garnet zone, through $540-565{ }^{\circ} \mathrm{C}$ and $5.8-5.9 \mathrm{kbar}$ in the staurolite zone, $525-600{ }^{\circ} \mathrm{C}$ and 6.0-7.3 kbar in the kyanite zone, and $670-715^{\circ} \mathrm{C}$ and $7.5-8.4 \mathrm{kbar}$ at the onset of the 
sillimanite zone (Dasgupta et al., 2004, Dubey et al., 2005; Dasgupta et al., 2009, Mottram et al. 2014b).

High-resolution $\mathrm{Lu}-\mathrm{Hf}$ garnet dates record a progressive increase in the age of garnet cores from low to high metamorphic grades in the LHS: 11.1-10.5 Ma in the garnet zone, 12.8 Ma in the staurolite zone, 13.7 Ma in the kyanite zone, 14.6 Ma in the sillimanite zone and 16.8 Ma in the sillimanite/K-feldspar zone (Anczkiewicz et al. 2014). Concordantly, phase equilibria modelling suggests that there was a decrease in the $P$ of initial garnet growth with increasing metamorphic grade such that the $T$ required for initial garnet growth was reached earlier in time for rocks buried closer towards the MCTZ (Gaidies et al. 2015). Peak metamorphism is interpreted to have been achieved approximately simultaneously in all zones over a short time span at 13-10 Ma, with a maximum duration of Barrovian metamorphism in Sikkim of 6-7 Myr (Anczkiewicz et al., 2014; Chakraborty et al., 2016).

A garnet-grade metapelite from the LHS (sample 24-99) was selected as the principal sample for this study, having been studied previously by Dasgupta et al. (2009), Anczkiewicz et al. (2014) and Gaidies et al. (2015). Field observations pertaining to this study suggest that 24-99 reflects a slightly higher metamorphic grade than previously assumed, well inside the garnet zone and approximately $400 \mathrm{~m}$ south of the first appearance of staurolite (Fig. 2.1).

\subsection{Textural and compositional characterisation}

\subsubsection{Methods}

\section{Wavelength-dispersive X-ray fluorescence analysis}

The whole-rock chemical composition of sample 24-99 was obtained using wavelengthdispersive X-ray fluorescence analysis (WDXRFA) using the Philips PW-2400 instru- 
ment at the University of Ottawa. Given the macroscopic textural uniformity of the sample and the relatively small grain sizes of garnet, $\sim 0.5 \mathrm{~kg}$ of the rock was crushed and ground to prepare powders for glass discs.

\section{Whole-rock X-ray $\mu$-computed tomography}

A SkyScan 1173 high-resolution X-ray $\mu$-computed tomography (XR $\mu$-CT) scanner located in the Department of Earth Sciences at Carleton University (Ottawa, Canada) was used to characterise the spatial, size and shape distribution of garnet within the study sample. Scanning was conducted using a $130 \mathrm{kV} / 61 \mu \mathrm{A}$ X-ray source and a $0.25 \mathrm{~mm}$ thick brass filter, with an exposure time of $1300 \mathrm{~ms}$. In order to fully image the sample, it was rotated within the conical X-ray beam in a step increment of $0.25^{\circ}$, over a total $360^{\circ}$ rotation. Two-dimensional (2D) attenuation slices of the scanned volume were acquired, the thickness of each slice corresponding to $13.15 \mu \mathrm{m}$. To reduce processing time, every second slice was used for reconstruction, reducing the three-dimensional (3D) spatial resolution of the dataset to $26.3 \mu \mathrm{m}$ per voxel edge. Following rendering of these grayscale slices into a 3D image using SkyScan NRecon software, the computational software Blob3D (Ketcham, 2005) was used in order to quantify the size, abundance and central coordinates of in situ garnet porphyroblasts. This permitted the extraction of the garnet crystal size distribution (CSD) for 24-99, in addition to providing high-quality textural information such as crystal volumes, surface areas and crystal shape parameters.

\section{SELFRAG ${ }^{T M}$ sample preparation, X-ray $\mu$-computed tomography of mounted garnet, and central sectioning}

In order to most accurately characterise the compositional properties of the garnet population, sections through the geometric centres of crystals representative of the 
entire garnet CSD were prepared. To isolate garnet crystals for central sectioning, the Selfrag ${ }^{T M}$ high-voltage pulse power fragmentation facility at the Department of Geological Sciences and Geological Engineering of Queen's University (Kingston, Canada) was used. A fist-sized sample was shocked five times with 50 pulses of 120$140 \mathrm{kV}$ current. The electrode gap was set to $20 \mathrm{~s}$. Intact crystals with little notable resorption that were representative of the CSD were then picked and mounted into grain mounts, and scanned with the SkyScan 1173 XR $\mu$-CT instrument at Carleton University. Scan settings utilised a $1300 \mathrm{~ms}$ exposure time and a $70 \mathrm{kV} / 114 \mu \mathrm{A}$ Xray source with a $0.25 \mathrm{~mm}$ thick aluminium filter. This yielded image slices with a resolution between 6.04 and $7.82 \mu \mathrm{m}$.

Tomography datasets were then used in order to locate geometric cores of garnet parallel to $\{011\}$ dodecahedral planes, which were exposed by hand polishing and continuous comparison with $\mu$-CT image slices, yielding accuracy of within $\pm 25 \mu \mathrm{m}$ of the core. Attempts were made to ensure that all sections were made parallel to the elongation direction of the dodecahedra, however uncertainties with respect to sectioning effects, garnet geometry and orientation cannot be completely excluded.

\section{Electron probe microanalysis}

Quantitative compositional profiles of centrally sectioned garnets were obtained on a Cameca Camebax MBC in the Department of Earth Sciences at Carleton University (Ottawa, Canada) that is fitted with a wavelength-dispersive (WDX) analytical system. Profiles were collected along the longest possible axis through the geometric core at a $20 \mu \mathrm{m}$ analysis spacing. Operating conditions for all traverses were $20 \mathrm{kV}$ accelerating potential with a $20 \mathrm{nA}$ beam current. Peak counting times for analysed elements were either $40 \mathrm{~s}$ or 62,500 accumulated counts for $\mathrm{Si}, \mathrm{Ti}, \mathrm{Al}, \mathrm{Fe}, \mathrm{Mn}, \mathrm{Mg}$ and Ca. Background measurements were made at $50 \%$ peak counting times on each side 
of the analysis peak. Calibration was to a range of natural and synthetic standards, and counts were converted to elemental wt.\% by the Cameca PAP matrix correction procedure.

Compositional maps of $\mathrm{Fe}, \mathrm{Mg}, \mathrm{Mn}$ and $\mathrm{Ca}$ in garnet were obtained on a JEOL 8230 SuperProbe at the University of Ottawa. Maps were produced using a focused acceleration voltage at $20 \mathrm{kV}$, a beam current of $100 \mathrm{nA}$ and a dwell time of $200 \mathrm{~ms}$. Maps have a spatial resolution of $3 \mu \mathrm{m}$.

\subsubsection{Results}

\section{Petrography}

Sample 24-99 comprises garnet, muscovite ( $\sim 35 \%)$, quartz $(\sim 38 \%)$, chlorite $(\sim 9 \%)$, biotite $(\sim 8 \%)$, plagioclase $(\sim 6 \%)$, and ilmenite with accessory apatite, allanite, tourmaline and graphite (Fig. 2.2). The alignment of muscovite, chlorite and graphite defines a penetrative matrix foliation, $S_{1}$, which is microfolded to form a spaced $S_{2}$ crenulation cleavage (Fig. 2.2a).

Garnet is largely xenoblastic (although subhedral porphyroblasts are also present) and contains inclusions of ilmenite, apatite, allanite and flattened quartz grains (Fig. $2.2 \mathrm{~b})$. These inclusions form sinusoidal inclusion trails that are largely concordant with the external foliation, suggesting that garnet growth was syn-kinematic with respect to $\mathrm{D}_{2}$ deformation. Biotite is largely aligned with the foliation. Chlorite is present as small matrix-aligned laths and as ragged clots that embay into garnet grains (Fig. 2.2 b), with the latter interpreted as a product of minor retrogression. There is also a notable decrease in the mode of chlorite aligned with $\mathrm{S}_{2}$ in the vicinity of garnet-rich areas. 


\section{Garnet shape and size distribution}

A volume of ca. $37 \mathrm{~cm}^{3}$ of 24-99 was scanned and shown to contain 8173 garnet porphyroblasts, which occupy ca. 2.4 vol.\% of the total rock volume (Fig. 2.3a \& b). The CSD is log-normal and unimodal, with a skewness of 0.8 (Fig. 2.3c), and is detailed in Appendix 2.1. The maximum and minimum grain radii are $0.71 \mathrm{~mm}$ and $0.053 \mathrm{~mm}$ respectively, the mean radius is $0.24 \mathrm{~mm}$, and the average $3 \mathrm{D}$ aspect-ratio of the segmented in situ garnet population is 1.5. Crystals commonly exhibit faceted surfaces in the reconstructed $\mu$-CT volumes (Fig. 2.3d). Fifteen percent of the garnets are in mutual contact.

\section{Whole rock chemistry and matrix mineral compositions}

Table 2.1 displays the whole-rock major element WDXRF results for sample 24-99, together with the corresponding bulk rock composition used for the numerical simulations of garnet crystallisation conducted in this study. Biotite throughout the matrix is chemically unzoned $\left(X_{\mathrm{Mg}}\left[\mathrm{Mg} /\left(\mathrm{Mg}+\mathrm{Fe}^{2+}\right)\right]=0.41-0.46, \mathrm{PFU}_{\mathrm{K}} \sim 0.85\right)$. Prograde matrix chlorite contains $X_{\mathrm{Mg}}=0.46-0.47$, whereas retrograde chlorite surrounding garnet is slightly less Mg-rich, with $X_{\mathrm{Mg}}=0.42-0.44$. Plagioclase is not systematically zoned $\left(X_{\mathrm{Ca}}[\mathrm{Ca} /(\mathrm{Na}+\mathrm{Ca})]=0.16-0.20\right)$. Representative matrix mineral analyses are given in Appendix 2.2.

\section{Garnet compositional zoning}

Multiple crystals from each size class were centrally sectioned and compositionally analysed, and representative profiles for grains in CSD classes 1, 3, 6, 8, 10, 12, 14 and 16 (where the largest and smallest garnets belong to size class 1 and 20, respectively; Fig. 2.3.) are displayed in Fig. 2.5. X-ray compositional maps are presented in Fig. 2.4, and full line profiles can be found in Appendix 2.3. The smallest docu- 
mented grains, in CSD classes 17 to 20, could not be identified in the disaggregated volume, and perhaps were more susceptible to destruction during the fragmentation process. Prior to exposure of the geometric core, polishing of several crystals was stalled approximately $25 \%$ through the crystal, to allow for compositional analysis. Assessment of these non-central sections in conjunction with subsequently acquired central sections supports the presence of a single geochemical core that is congruent with the geometric core.

Compositional profiles for all divalent cations and results from X-ray mapping confirm that the zoning of $\mathrm{Mn}, \mathrm{Mg}, \mathrm{Fe}$, and the first order shape of Ca-zoning is radially symmetric about the centre of each crystal. In the case of all major cations, rim compositions of all grains independent of size are within error of each other. $X_{\mathrm{prp}}$ $\left[=\mathrm{Mg} /\left(\mathrm{Fe}^{2+}+\mathrm{Ca}+\mathrm{Mg}+\mathrm{Mn}\right)\right]$ displays systematic core to rim concentric zoning, forming smooth, concave-up parabola (Fig. 2.5 and Fig. 2.4. $X_{\text {prp }}$ of the core of the largest garnet is 0.04 , and progressively smaller grains contain increasingly elevated $X_{\text {prp }}$ contents in their cores to a maximum in the smallest grain of 0.05 . Rims of all grains contain $X_{\text {prp }} \sim 0.09$. Grains of size classes 1 to 6 contain smooth core plateaus of minimum $X_{\text {prp }}$, while smaller grains exhibit increasingly steep 'V-shaped' rim-to-rim profiles. $X_{\text {alm }}\left[=\mathrm{Fe}^{2+} /\left(\mathrm{Fe}^{2+}+\mathrm{Ca}+\mathrm{Mg}+\mathrm{Mn}\right)\right]$ zoning displays similar systematic behaviour: the cores of the largest and smallest documented garnet grains contain $X_{\text {alm }}$ contents of 0.67 and 0.72 , respectively (Fig. 2.5b).

$X_{\text {sps }}\left[=\mathrm{Mn} /\left(\mathrm{Fe}^{2+}+\mathrm{Ca}+\mathrm{Mg}+\mathrm{Mn}\right)\right]$ decreases from core to rim in all grains, exhibiting typical bell-shaped prograde zoning (Fig. 2.5k), with $X_{\mathrm{sps}}$ isopleths that are parallel to the euhedral crystal surfaces (Fig. 2.4). The largest and smallest grains contain $X_{\mathrm{sps}}$ contents of 0.125 and 0.06 in their cores, respectively, between which there is a progressive decrease in core $X_{\text {sps }}$ from large to small grains. Grains of size classes $1,3,6,8,12,14$ and 16 clearly follow this trend. Size class 10 contains lower 
$X_{\text {sps }}$ and higher $X_{\text {alm }}$ contents than might be expected given the observed relationship between crystal size and core composition.

Unlike the rather smooth core-rim distributions of other major elements, compositional trends of $X_{\text {grs }}\left[=\mathrm{Ca} /\left(\mathrm{Fe}^{2+}+\mathrm{Ca}+\mathrm{Mg}+\mathrm{Mn}\right)\right]$ are more complex. Superimposed on broad core-to-rim decreases in $X_{\text {grs }}$ (Fig. 2.5d), secondary short-wavelength oscillations $<10 \mu \mathrm{m}$ wide are present in porphyroblasts of all sizes, particularly observable near the rims of crystals (Fig. 2.4). A patchy, non-systematic Ca distribution is present in the cores of crystals. Consistent with the reports in some studies (Finlay \& Kerr, 1979; Carlson, 1989, Chernoff \& Carlson, 1997), documented $X_{\text {grs }}$ oscillations are not correlative with equal points within differently sized grains, such that the anomalous behaviour of $\mathrm{Ca}$ is demonstrably not a consequence of some simultaneous rock-wide event (e.g., change in fluid composition, $P$, or $T$ ).

In all mapped crystals, these systematic core-to-rim changes are accompanied by compositional sector zoning of $\mathrm{Ca}$ and Fe (dotted boxes, Fig. 2.4), associated with differential partitioning between the $\{110\}$ dodecahedral and $\{211\}$ trapezohedral garnet surfaces (Kohn, 2004, Stowell et al., 2011).

\section{Radius-rate method}

Comparison of incremental changes in compositional gradients using the radius-rate method provides the opportunity to graphically assess the relative influences of interface reaction and intergranular diffusion on the rate of crystal growth, as described by Kretz (1974) and Carlson (1989). Application of this method allows comparison of crystal radius, $r$, with the distance between two given compositional contours $\left(X_{1}\right.$ and $X_{2}$ ), $\Delta r$, for a range of crystal sizes (Fig. 2.6). If it is assumed that intracrystalline diffusion in garnet was negligible, then these radius-rate relationships may yield a measurement of the amount of material added to a crystal surface over a 
given compositional interval, a proxy for time passed (Daniel \& Spear, 1998). These measurements can be taken for a suite of garnet compositions normalised to the largest length scale of the dataset.

Following Kretz (1974), Carlson (1989) and Daniel \& Spear (1998), predicted radius-rate curves for interface- and diffusion-controlled growth are plotted in Fig. 2.7. Interface-controlled growth, whereby material is added to all crystal surfaces at the same radial-rate independent of crystal size yields a horizontal line, while diffusion-controlled growth yields a concave-up curve, with smaller crystals expected to grow with a faster radial-rate than larger ones. To allow for accurate determination of compositional changes, profiles of $X_{\mathrm{prp}}, X_{\mathrm{sps}}$ and $X_{\mathrm{alm}}$ were fit with cubic spline functions, which were then sampled at increments of 0.05 (excluding the outermost and innermost $10 \%$ of the crystal radius). The fluctuating nature of $X_{\text {grs }}$ makes it unsuitable for this analysis.

Selected represented radius-rate data for sample 24-99 is plotted in Fig. 2.7. whereby a single growth increment in all crystals is plotted for each end-member $\left(X_{\mathrm{prp}}: 0.07\right.$ and $0.08, X_{\mathrm{sps}}: 0.02$ and 0.03 and $X_{\mathrm{alm}}: 0.765$ and 0.775 ). These results plot close to, but below the radius-rate relationship expected for interface-controlled growth, with a slight negative correlation between crystal size and distance between compositional contours. This possibly indicates a decrease of the radial crystal growth rate for smaller crystals, relative to larger ones. Results are entirely inconsistent with those expected for growth limited by the rate of intergranular diffusion. The chemical analyses of multiple similar-sized grains indicate that the steepening of compositional gradients with a decrease of grain size is representative of the population and is independent of the positions of the grains in the rock. 


\subsection{Numerical simulations of garnet crystallisation}

In order to provide a framework for the garnet crystallisation modelling conducted in this study, phase equilibria have been predicted using the Gibbs energy minimisation software Theriak/Domino (de Capitani \& Brown, 1987, de Capitani \& Petrakakis 2010). Modelling was conducted in the 11-component system $\mathrm{MnO}-\mathrm{Na}_{2} \mathrm{O}-\mathrm{CaO}-\mathrm{K}_{2} \mathrm{O}-$ FeO-MgO- $-\mathrm{Al}_{2} \mathrm{O}_{3}-\mathrm{SiO}_{2}-\mathrm{CO}_{2}-\mathrm{H}_{2} \mathrm{O}-\mathrm{TiO}_{2}$, using the bulk rock composition given in Table 2.1. Activity-composition $(a-X)$ relationships of the end-member phases are those recommended by Holland \& Powell (1998). In addition, feldspar was modelled with a ternary solid solution (Baldwin et al., 2005), garnet as a quaternary solution following that of Connolly (2005), biotite utilises the model of White et al. (2005), chloritoid solid solution follows White et al. (2000) with the addition of the Mnbearing end-member, and chlorite follows that of Gaidies et al. (2008). White mica utilises Coggan \& Holland (2002), and Holland \& Powell (1998) was extended to consider the Mn-staurolite end-member. $\mathrm{No} \mathrm{Fe}_{2} \mathrm{O}_{3}$ was considered given the negligible amounts of $\mathrm{Fe}^{3+}$ in garnet and ilmenite. The metamorphic fluid was modelled as a carbon-, oxygen- and hydrogen-bearing $(\mathrm{COH})$ phase produced by the equilibration of $\mathrm{H}_{2} \mathrm{O}$ and excess graphite according to Connolly \& Cesare (1993). In addition to graphite saturation, the system was considered to be saturated with respect to $\mathrm{SiO}_{2}$ and $\mathrm{H}_{2} \mathrm{O}$ over the $P-T$ range of interest.

Using this approach, the peak metamorphic assemblage containing garnet, muscovite, chlorite, plagioclase, quartz and ilmenite (highlighted in red in Fig. 2.8) is predicted to be stable in a narrow wedge from relatively low- $P$ to 7.5 kbar between 520 and $575{ }^{\circ} \mathrm{C}$.

The nucleation and growth simulations discussed below utilise the same model system, and relevant input files are detailed in Appendies 2.4 and 2.5. 


\subsubsection{Nucleation of garnet}

\section{Parallel \& common tangent approach}

Given the compositional patterns observed in the garnet population of 24-99, the oldest portion of garnet in the rock is considered to correspond to the geochemical core containing the highest $X_{\mathrm{sps}}$, in this case in the largest garnet crystal. In the absence of diffusional modification of the garnet chemistry, this core composition may reflect the conditions of initial garnet nucleation during metamorphism. In such a case when diffusion across a nucleus is negligible and the nucleus composition is preserved during metamorphism, the parallel tangent method (schematically outlined for a twocomponent system in Fig. 2.9p, may be applied to estimate the initial conditions of garnet nucleation (see also Gaidies et al., 2011; Pattison et al., 2011; Spear et al., 2014; Gaidies, 2017). The chemical driving force for nucleation, $\Delta G_{V}$, is maximised if the difference in chemical potentials between the metastable reacting system and nucleus of the components involved in the nucleation are identical. The composition of the nucleus, $X_{\text {grt }}^{\text {nuc }}$, can then be determined by the point of tangency on the nucleating product $G-X$ function of a line that is parallel to the tangent that corresponds to the energy state of the reactant. As can be seen in Fig. 2.9, $X_{\text {grt }}^{\text {nuc }}$ differs from the equilibrium composition, $X_{\mathrm{grt}}^{\mathrm{eqm}}$, and this difference increases as the departure from equilibrium, and hence $\Delta G_{V}$, grows. Nucleation in natural systems is thought to be a partitioning process (e.g., Gaidies, 2017), depleting the bulk system with respect to the components fractionated into the nucleus. As a consequence, the parallel tangent method should only be used to predict the composition of the first nucleus, prior to chemical diffusion.

Given the small sizes of nuclei at the nm scale, it may however be reasonable to assume that diffusion across the nucleus and across its interface with the react- 
ing system may be efficient enough to establish the equilibrium composition of the

product, $X_{\mathrm{grt}}^{\mathrm{eqm}}$. This composition corresponds to the point on the $G-X$ function of the reaction product that is mutual tangential with the $G-X$ function of the reacting system. This approach constitutes the common tangent method inherent in the widely applied equilibrium model. The molar Gibbs energy difference between the initial and final equilibrium states, $\Delta G_{r}$, is the chemical driving force for the entire reaction. It depends on the bulk composition of the system and reaches a maximum if the bulk composition approaches $X_{\mathrm{grt}}^{\mathrm{eqm}}$.

\section{Conditions of nucleation}

The parallel and common tangent methods outlined above can be considered endmember approaches for the prediction of the properties of a nucleus and the $P-T$ conditions of nucleation. Figure 2.10 illustrates the differences in the $P-T$ conditions determined for nucleation using both the parallel and the common tangent methods for the bulk rock composition of 24-99 given in Table 2.1. Whereas solid contours in Fig. 2.10a-d correspond to $X_{\text {grt }}^{\mathrm{eqm}}$, the colour-coded $P-T$ fields reflect $X_{\mathrm{grt}}^{\mathrm{nuc}}$.

As can be seen, the differences between the two end-member scenarios tend to increase with departure from the equilibrium $P-T$ conditions of initial garnet crystallisation (i.e. on the garnet-in line). Fig. 2.10 compares the $P-T$ conditions of nucleation obtained with the common and parallel tangent methods using the core composition of the largest garnet crystal in the rock. The area of $X_{\text {grt }}^{\text {nuc }}$ isopleth intersection clusters around conditions of $\sim 527^{\circ} \mathrm{C}$ and $\sim 4.8 \mathrm{kbar}$. The $X_{\mathrm{grt}}^{\mathrm{eqm}}$ isopleths intersect at a point positioned on the equilibrium garnet-in curve at $\sim 519{ }^{\circ} \mathrm{C}$ and 4.4 kbar. Given that the equilibrium model perfectly predicts the observed garnet core composition, and considering that the isopleths determined through the parallel tangent method deviate slightly from those observed (resulting in an area of isopleth 
intersection rather than a point), the application of the equilibrium model in the following simulations is justified. In any case, the differences in nucleation conditions between the equilibrated and maximum overstep scenarios amount to $0.4 \mathrm{kbar}$ and $8{ }^{\circ} \mathrm{C}$, within the error associated with the thermodynamic modelling and geochemical analysis (Powell et al., 1998; Palin et al., 2016).

In both scenarios, garnet is predicted to nucleate in an assemblage composed of plagioclase, muscovite, chlorite, ilmenite, quartz, graphite and a $\mathrm{COH}$-fluid. Fig. 2.10f illustrates how $\Delta G_{V}$ depends on $P-T$ for garnet nucleation in 24-99, with a maximum driving force for nucleation of $X_{\mathrm{grt}}^{\mathrm{eqm}}$ on the order of $2.5 \mathrm{~kJ} \mathrm{~mol}^{-1}$ (per 12 oxygen garnet).

\subsubsection{Nucleation and growth of garnet}

Given that the differences between garnet core compositions predicted with the common and parallel tangent methods are small, and since the equilibrium model results best match the observed core composition, the THERIA_G model (Gaidies et al., 2008) is applied in this study to simulate the metamorphic crystallisation of the entire garnet population in 24-99. The theoretical background of the simulation is outlined and modelling results are presented below. It is important to note that the precise numerical values discussed are a result of the numerical precision of the model and are not intended to imply lack of uncertainty on these numbers. However the relative $P-T$ estimates and paths, nucleation histories and metamorphic heating rates are of geological relevance.

\section{Garnet crystallisation simulations with THERIA_G}

THERIA_G simulates garnet growth as the successive addition of concentric shells, where crystal centres are progressively distributed with a density defined by a relative 
CSD (as illustrated in Fig. 2.11). This relative CSD contains the observed density of crystals in each class and the CSD bin size ( $x$ in Fig. 2.11).

For some input $P-T-t$ path with initial conditions constrained by equilibrium isopleth intersection, THERIA_G iteratively searches for the $P-T$ increment that yields the calculated garnet volume required to distribute a radial increment of $\Delta s$ on all garnet individuals in the simulation. At each point in the simulation, this volume is calculated based on Gibbs energy minimisation at the respective $P-T-X$ condition assuming thermodynamic equilibrium between the new garnet shell and the rock matrix. As a consequence of this volume distribution, all garnet crystals are simulated to grow with a radial rate that is independent of crystal size, consistent with the geometric implications of interface-controlled crystal growth (Kretz, 1974 , Chernoff \& Carlson, 1997, Spear \& Daniel, 1998, Gaidies et al., 2008). Once a population of early growing crystals has sufficient shells such that their sizes exceed the CSD bin width, a new population of crystals is nucleated. This approach ensures that the correct relative proportions of the observed CSD are reproduced. However, the absolute radii of garnet crystals are governed by the simulated $P-T-t-X$ path of the rock, and are not an input for the simulations. Consequently it is possible for garnet crystal sizes to differ from those observed, and the number of different size classes of the calculated garnet population may be smaller than that of the rock. Since the predicted volume of garnet depends on the modelled $P-T-t-X$ trajectory, it can be used together with the predicted chemical composition of garnet and mineral content of the rock to evaluate the applicability of the crystallisation simulation to the sample.

In order to account for chemical fractionation due to garnet growth, THERIA_G subtracts the components required for each episode of shell growth from the bulk composition, yielding a changing effective bulk composition (EBC) for sequential volume 
searches. Consequently, if steps in $P-T$ space are sufficiently small, Rayleigh fractionation behaviour is approximated. To also account for the intracrystalline diffusion of $\mathrm{Mn}, \mathrm{Fe}, \mathrm{Mg}$ and $\mathrm{Ca}$ in garnet, multicomponent diffusion between garnet shells is simulated at each growth step. The kinetic parameters obtained by Chakraborty \& Ganguly (1992) were applied, and following the approach of Loomis $(1982)$ and Florence \& Spear (1991), the tracer diffusion coefficient of $\mathrm{Ca}, \mathrm{D}_{\mathrm{Ca}}$, was defined as $\mathrm{D}_{\mathrm{Fe}} / 2$.

THERIA_G therefore models progressive episodic nucleation of successive garnet generations, with the largest size class nucleating first in the crystallisation sequence. By increasing the number of size classes, THERIA_G distributes more nucleation pulses along the $P-T-t$ path, and simulates nucleation in an increasingly continuous manner. For the heating scenarios considered in the simulations presented here, 20 generations were chosen to describe the CSD (Fig. 2.5 ) in which the largest grains are found within size class 1 , and the smallest crystals within size class 20. Simulations with more than 20 size classes do not yield different results.

For the modelled size-independent radial growth rates, in the early stages of crystallisation, only a small change in $P-T$ is needed in order to grow the equilibrium volume required on all porphyroblasts. Consequently, large early-nucleated crystals develop shallow compositional gradients in their cores. However, the volume required for continued radial growth increases according to the constraints defined by the spherical geometry of the model crystals, yielding larger steps in $P-T$, and progressively steepening chemical gradients in the cores of later nucleating crystals. As a consequence, relatively late nucleated garnet is more susceptible to diffusional relaxation than earlier nucleated larger grains. For this reason, the chemical zoning developed in the larger crystals of the garnet population may be used to infer the evolving $P-T$ conditions of garnet crystallisation, and the zoning observed in the 
smaller grains may be used to constrain the rate with which $P-T$ may have changed during metamorphism. In the simulations presented here, rate modelling was fit to crystals in size classes 14 and 16, i.e. garnet with radii less than $\sim 0.3 \mathrm{~mm}$, whereas $P-T$ path modelling was fit primarily to the largest grains in size class 1 . Dwell times at peak metamorphism were not incorporated into final models, as they have been shown to have had negligible effect on the chemical zoning predicted by Gaidies et al. (2015).

Given that the THERIA_G approach is based on the thermodynamic equilibration between the outermost garnet shell and the rock matrix during garnet growth, the modelling of the nucleation and growth of the population can be considered a succession of equilibrium states where the interior of garnet is the only volume of the rock with potentially non-equilibrium properties, as determined by the intracrystalline diffusion simulations. For further discussion of THERIA_G see Gaidies et al. (2008).

\section{Results of crystallisation simulations}

$\mathbf{P}-\mathbf{T}$ path According to the calculations, growth zoning observed within the garnet population of 24-99 can best be described by crystallisation of garnet along the $P-T$ path shown in Fig. 2.12a, yielding compositional profiles illustrated in Fig. 2.13. Garnet crystallisation is predicted to start at $518{ }^{\circ} \mathrm{C}$ and $4.5 \mathrm{kbar}$ and finish at 564 ${ }^{\circ} \mathrm{C}$ and 5.6 kbar. The predicted path is approximately linear from the first appearance of garnet to peak conditions, and does not include a $10{ }^{\circ} \mathrm{C}$ period of isobaric $T$ increase previously inferred by Gaidies et al. (2015). This discrepancy between these new findings and those of Gaidies et al. (2015) likely reflects the fit between predicted and observed garnet growth zoning required for the larger compositional and microstructural dataset available in the current study. The older study is based 
on the compositional zoning observed in a single crystal.

The radius $-T$ relationship for the largest, earliest nucleated grain in the study is consistent with rapid early growth from 518 to $\sim 526{ }^{\circ} \mathrm{C}$, followed by an approximately linear radius increase to peak $P-T$ (Fig. 2.12 b). Along the $P-T$ path, garnet crystallised in an assemblage containing plagioclase, chlorite, muscovite, quartz, ilmenite, graphite and $\mathrm{COH}-f l u i d$. In the simulations excess graphite buffers the composition of the coexisting fluid such that $\mathrm{a}_{\mathrm{H}_{2} \mathrm{O}}$ varies between 0.85 and 0.91 along the $P-T$ path. Calculated garnet mode at peak conditions (2.5 vol.\%) is effectively identical to the volume of garnet documented using the XR $\mu$-CT dataset $(\sim 2.4$ vol.\%). The assemblage is calculated to change at $555^{\circ} \mathrm{C}$ and $5.3 \mathrm{kbar}$, where staurolite becomes part of the stable assemblage for the final $\sim 10{ }^{\circ} \mathrm{C}$ of garnet growth (Fig. 2.12 ). Staurolite is not observed in the sample, however a volume of 0.4 vol. $\%$ is predicted to form along the total $P-T$ path. Simulations that exclude staurolite as a possible phase during metamorphism have no influence on the predicted garnet zoning and size, and the late-stage onset of staurolite crystallisation as predicted by the phase equilibria is consistent with the proximity of the sample to the staurolite-in isograd (Fig. 2.1).

At peak $P$, phase equilibria modelling of the EBC indicates that the stable assemblage does not contain biotite (Fig. 2.12 a), despite its presence as a minor foliation defining phase in sample 24-99. For biotite to form as observed, $P$ had to decrease $\sim 0.1$ kbar subsequent to peak $P$, crossing the upper stability limit of the biotitebearing assemblage at approximately $5.4 \mathrm{kbar}$, using an EBC after fractionation of garnet. The growth of biotite as a foliation forming phase subsequent to the conclusion of garnet crystallisation at these conditions is not unreasonable, as the high entropy system near peak conditions will still be actively deforming. Adding this portion of the $P-T$ path to the numerical simulation does not affect the produced 
chemical zoning in garnet. Given the presence of a biotite-grade Barrovian zone at sub-garnet conditions (e.g., Dasgupta et al., 2009; Mottram et al., 2014a), a lack of biotite along the prograde path is likely a consequence of local bulk compositional effects in the sample. This seems reasonable given the absence of biotite inclusions in garnet and its relatively low modal percentage.

Nucleation history of garnet As a consequence of chemical fractionation associated with garnet growth in the simulations, each discrete size class of garnet is predicted to nucleate at equilibrium in a different EBC (Fig. 2.12 a). Figure 2.12p illustrates the distribution of the predicted nucleation pulses along the modelled $P-T$ path. According to the calculations, size classes $1-10$ nucleate between $518{ }^{\circ} \mathrm{C}$ and $520{ }^{\circ} \mathrm{C}$, after which nucleation events are increasingly distributed along the $P-T$ path, with the final episode of nucleation at $552{ }^{\circ} \mathrm{C}$ before cessation of garnet growth at 564 ${ }^{\circ} \mathrm{C}$. Accordingly, migration of the garnet-in curve for early nucleated size classes is negligible: size classes 1-10 nucleate in very similar chemical systems, with significant efficacy of EBC modification only once appreciable volumes of garnet have formed, approximately after nucleation of size class 11 (Fig. 2.12 ). This approximately isothermal nucleation of size classes 1-10 yields a very rapid early nucleation rate (dN/dt, Fig. 2.12k) followed by a slowing of the nucleation rate as the crystallisation interval proceeds.

Rates of metamorphism Model compositional results displayed for the described $P-T$ path are for heating rates of $50{ }^{\circ} \mathrm{C} \mathrm{Myr}^{-1}$ (dashed lines, Fig. 2.13), $100{ }^{\circ} \mathrm{C}$ $\mathrm{Myr}^{-1}$ (solid lines, Fig. 2.13) and $300{ }^{\circ} \mathrm{C} \mathrm{Myr}^{-1}$ (dotted lines, Fig. 2.13). For size classes most sensitive to diffusion (14 and 16), excellent fits to the observed major element chemistry can be achieved when using heating rates exceeding $100{ }^{\circ} \mathrm{C} \mathrm{Myr}^{-1}$ along the best-fit $P-T$ path. The core composition of the largest crystal can be 
well constrained for any heating rate with a good fit between the modelled and the observed chemistry. At rates exceeding $100{ }^{\circ} \mathrm{C} \mathrm{Myr}^{-1}$, modelled core compositions of $X_{\mathrm{prp}}$ and $X_{\mathrm{grs}}$ are consistent with the observed compositions of all garnet sizes. Initial $X_{\mathrm{sps}}$ and $X_{\mathrm{alm}}$ in garnet cores are similarly well matched for size classes 1-8 and $12-16$ at these rates.

Compositional zoning in garnet Given peak metamorphic conditions of $564{ }^{\circ} \mathrm{C}$ and $5.6 \mathrm{kbar}$ and the input of a CSD containing 20 size classes (Fig. 2.5k), the observed rim compositions for all crystal sizes are reproduced in the simulations for each major cation. The crystallisation model therefore successfully simulates the formation of a garnet population with absolute crystal sizes equivalent to those documented in the sample.

For all size classes, modelled $X_{\text {grs }}$ is more sensitive to minor variations in $P-T$ path when compared to $X_{\mathrm{sps}}, X_{\mathrm{prp}}$, and $X_{\text {alm }}$, with significant oscillation when $P$ and $T$ evolves in a stepwise manner. However, given the spatial discordance between $\mathrm{Ca}$ oscillations in different grains, these oscillations are not interpreted to record a rockwide event or a change in extrinsic conditions, as has been previously suggested for compositional oscillations (Finlay \& Kerr, 1979; Carlson, 1989, Shore \& Fowler, 1996 Kohn, 2004). Instead, similar to conclusions made by Chernoff \& Carlson (1997), fluctuations in $X_{\text {grs }}$ zoning are instead interpreted to suggest that Ca may not have been fully equilibrated at or along the garnet/matrix interfaces during crystallisation. Consequently, the chosen $P-T$ path successfully models the long wavelength core-torim $\mathrm{Ca}$ variation, and does not attempt to account for short wavelength oscillations with rock-wide $P-T$ steps.

Whereas the predicted $X_{\mathrm{sps}}$ content at the start of crystallisation of these generations matches the observed composition well, modelled $X_{\mathrm{sps}}$ in wider core regions 
of the oldest garnet individuals (size classes 1,3 and 6) exhibits homogeneous core plateaus, discordant to the observed steep, 'bell-shaped' compositional profiles. Similar discordance is observed for $X_{\text {alm }}$, with the simulation under-predicting the steepness of core gradients relative to those observed. These disparities in compositional gradients between modelled and observed garnet grains are absent in crystals of size classes 8-16, i.e. in crystals with a radius less than $0.45 \mathrm{~mm}$.

Furthermore, the modelled core composition of size class 10 is notably discordant with the measured compositions for all grains of this size class. Measured $X_{\mathrm{sps}}$ and $X_{\text {alm }}$ are $\sim 0.05$ lower and higher, respectively, than predicted for the best-fit $P-T-t$ path. Deviations of the model predictions from the observed $X_{\text {prp }}$ and $X_{\text {grs }}$ contents are negligible and both $X_{\text {prp }}$ and $X_{\text {grs }}$ rim compositions as well as crystal sizes are well predicted by the model.

Additional simulations were applied in order to test whether this discrepancy can be attributed to diffusional modification of the core compositions in size class 10 during the crystallisation interval, prior to nucleation of later garnet classes. Results from a range of simulated scenarios that incorporate slow heating rates $\left(10{ }^{\circ} \mathrm{C}\right.$ $\mathrm{Myr}^{-1}$ ) up to the conditions of nucleation of size class 12 at $\sim 522{ }^{\circ} \mathrm{C}$, followed by rapid $100{ }^{\circ} \mathrm{C} \mathrm{Myr}^{-1}$ to peak conditions do not yield different compositional results. At the conditions of nucleation and early growth of size class $10\left(\sim 520^{\circ} \mathrm{C}\right)$ intracrystalline diffusion is an ineffective homogeniser (e.g., Caddick et al., 2010), and we instead attribute some of this discrepancy to a combined effect of a lack of correspondence between the geochemical and geometric core and to the influence of enhanced intracrystalline diffusion in the vicinity of large mineral inclusions in the garnet core. 


\subsection{Discussion}

\subsubsection{The rate of metamorphism}

For heating rates exceeding $100{ }^{\circ} \mathrm{C} \mathrm{Myr}^{-1}$, the crystallisation model applied in this study reproduces the observed compositional gradients in the analysed grains. The compositional zoning in the smallest crystals cannot be matched if slower metamorphic rates are used in the simulations. Given the steepening of the average compositional gradient from large to small grains, this corresponds to a minimum linear radial growth rate of garnet in 24-99 of $1.4 \mathrm{~mm} \mathrm{Myr}^{-1}$. For the obtained rates of metamorphic heating, the modelling indicates that the largest grains reached peak metamorphic $P$ conditions $\sim 0.5$ Myr after the first appearance of garnet in the assemblage, consistent with the preservation of primary growth zoning and compositional sector zoning.

Sample 24-99 has been dated using Lu-Hf garnet geochronology yielding an age of $10.59 \pm 0.16 \mathrm{Ma}$ (Anczkiewicz et al., 2014). Given significant fractionation of Lu into garnet cores, this date is interpreted to represent the age of initial garnet growth, which is approximately contemporaneous with the attainment of peak metamorphic conditions in the Sikkimese Barrovian sequence. A kyanite-zone garnet with a radius of $1.5 \mathrm{~cm}$ from the same sequence yielded whole-rock isochron and garnet rim ages of $13.7 \pm 0.2 \mathrm{Ma}$ and $9.9 \pm 3.8 \mathrm{Ma}$, respectively, with the low precision of the latter

age a consequence of low isotopic concentrations in the rim regions Anczkiewicz et al., 2014). As with approximately contemporaneous initial garnet growth and peak conditions in the garnet zones, rapid garnet crystallisation is inferred in the kyanite zone from the unresolvable age differences between the Lu-rich core and several midcrystal fragments.

Consistent with results summarised by Viete \& Lister (2016), metamorphic rate 
estimates in Sikkim derived from the presented geospeedometric methods are significantly faster than equivalent geochronological constraints from Anczkiewicz et al. (2014), which provide a maximum duration of garnet growth of $\sim 7.8 \mathrm{Myr}$ in the kyanite zone. Whether this age difference is a consequence of the limited temporal resolution of geochronological methods, or uncertainties in the Arrhenius parameters and erroneous assumptions in diffusion models is beyond the scope of discussion here, but confirms the short- to extremely short-duration metamorphism experienced in the Sikkim Barrovian sequence. While extremely rapid, such fast rates of metamorphism are not uncommon in the geological record (e.g., Oliver et al., 2000; Faryad \& Chakraborty, 2005, Ague \& Baxter, 2007; Smye et al., 2011; Viete et al., 2011), and raise significant questions about the plausibility of crustal-scale heat conduction required by thermal relaxation over such short-duration $(<10 \mathrm{Myr})$ timescales.

\subsubsection{Departure from equilibrium during garnet crystallisation}

Most previous estimates of disequilibrium during metamorphism have been derived from studies of contact metamorphic rocks (e.g., Waters \& Lovegrove, 2002 Pattison \& Tinkham, 2009, Pattison et al., 2011), yielding estimates of thermal overstep between 30 and $80{ }^{\circ} \mathrm{C}$ (cf. Carlson et al., 2015). Medium-grade regionally metamorphosed rocks have historically been considered to be far less susceptible to developing significant reaction overstep compared to contact metamorphic rocks, with potentially only a few degrees of overstep $\left(<10^{\circ} \mathrm{C}\right)$ because of the abundant availability of catalysing fluid and typically slower heating rates (e.g., Walther \& Wood, 1984 Pattison et al. 2011). However, such lithologies are increasingly thought to contain a record of persistent disequilibrium during prograde metamorphism (Wilbur \& Ague, 2006; Pattison et al., 2011). Recent studies have suggested that reaction overstep during interface-controlled growth may have only a small dependence on heating rate 
(Waters \& Lovegrove, 2002), with a greater dependence on the variation of interfacial energy (Gaidies et al. 2011).

The equilibrium model is capable of predicting the compositional zoning from core to rim of most grains of the garnet population, and reproduces the observed garnet CSD and the garnet nucleation history. It may therefore be inferred that the range of $T$ overstep over the duration of the progressive garnet nucleation and growth interval in sample 24-99 was consistently negligible. Consequently, for some hydrous metapelitic assemblages within regional terranes such as in the LHS of Sikkim, the effects of inhibitive kinetics may not result in degrees of overstep that are distinguishable from the uncertainties inherent in the chemical datasets and models used, even for very rapid rates of metamorphism.

These results are in contrast to Kelly et al. (2013), who, in a diffusion-controlled numerical simulation of the nucleation and growth of garnet, constrained thermal overstep in regionally metamorphosed rocks to an average of $33{ }^{\circ} \mathrm{C}$ (range $5-67{ }^{\circ} \mathrm{C}$ ). Impediments to garnet crystallisation were thought to significantly retard nucleation and reaction, with the inferred consequence that nucleation overstep occurred multiples times through the interval of progressive crystallisation.

While strain energy is not treated explicitly in these models, petrologic evidence for significant strain in the microstructure of 24-99 is consistent with an interpretation of negligible departure from equilibrium. Deformation during the interval of crystallisation can significantly reduce the activation energy for nucleation either by contributing energy to the reactant assemblage (Stünitz, 1998) and by a reduction in the interfacial energy that inhibits nucleation, or both. This catalytic effect of deformation on nucleation (Cahn, 1957) may be critical to reducing overstep in metamorphosed rocks (e.g., Pattison \& DeBuhr, 2015, Gaidies, 2017). Similarly, the documented continuity of the CSD, coupled with the continuous Rayleigh nature of 
observed growth profiles [in contrast with 'flat-top' profiles such as those observed by Zeh (2006), for example], lends support to a scenario in which little disequilibrium was required to nucleate garnet. If there had been significant kinetic barriers to nucleation, overstep may have elevated the driving force for garnet nucleation, culminating in the eventual simultaneous nucleation of numerous crystals once the energy barrier was overcome. In such a case, one might expect a more negatively skewed CSD, contrary to that observed in this study.

\subsubsection{Effect of model assumptions}

Several assumptions made during the modelling procedure may contribute to some of the systematic discrepancies between the observed garnet dataset and the simulated profiles.

\section{Geometric assumption}

Whereas the compositional gradients of $X_{\text {prp }}, X_{\text {grs }}$ and $X_{\text {alm }}$ can largely be predicted from core to rim for every garnet size class in the calculations (Fig. 2.13), the observed compositional gradients in $X_{\mathrm{sps}}$ of particularly large crystals, rim-ward of their cores, deviate from those predicted. THERIA_G modelling consistently overestimates $X_{\mathrm{sps}}$ in these crystals. Some of the mismatch in these regions may stem from the model assumption of a spherical garnet geometry, as the idealised dodecahedra radius-volume relationship is different to that of a sphere (Fig. 2.14. Volume-radius relationships for a sphere and the inscribed sphere of a dodecahedra (as was measured with EPMA) both define a rapid early increase in radius with volume (resulting in the development of a compositional plateau). However, this increase is more rapid for the latter, yielding steeper core gradients in dodecahedra than in modelled spherical crystals for the same chemical system. 
This shape effect is likely to account for some of the mismatch between observed and modelled compositional zoning in large crystals. In the case presented here, the effect of making the assumption that garnet crystals are spherical is minimal because of fast rates of garnet growth and lack of diffusional homogenisation. However, in metamorphic rocks that experienced slower heating rates the spherical garnet assumption may result in the underestimation of the timescales of diffusional modification and metamorphism and should therefore be considered with caution.

The spherical assumption may also not be fully representative of crystal growth of garnet in the rock studied as it assumes isotropic radial crystal growth outwards of the nucleus. Compositional profiles through garnet, however, reveal some degree of growth anisotropy, as the long-axis of a garnet crystal yields shallower compositional gradients compared to shorter axes. Making this spherical assumption may in some cases produce steeper compositional gradients than observed along the profiled long axis.

\section{Volume distribution}

It is important to also note that in the THERIA_G simulations garnet volume is distributed onto individual spherical crystals according to the geometrical constraints of size-independent interface-controlled growth kinetics. This is justified based on theoretical considerations (Kretz, 1974), empirical evidence Kretz, 1993, Chernoff \& Carlson, 1997, Spear \& Daniel, 1998), statistical textual analysis of 24-99 that indicates a clustered garnet distribution rather than an ordered one Gaidies et al. 2015), and results of radius-rate methods applied in this study. The garnet volume distributed during each growth increment is derived from equilibrium calculations, and results in an approximately linear relationship between volume and $T$ (Fig. $2.12 \mathrm{~b}$ ). However if the volume- $T$ relationship cannot be reliably predicted by equilibrium 
thermodynamics and is instead non-linear, then the resulting radius- $T$ relationship of early-nucleated crystals will change accordingly and predicted core compositions may be altered.

\section{Thermodynamic datasets}

The results of the calculations are dependent on the thermodynamic dataset and $a-X$

models chosen. However, extensive testing of the dataset with numerous metapelitic samples from Sikkim indicates that the chosen combination is the most effective at consistently reproducing the observed assemblages (Gaidies et al., 2015).

The observation that misfits are only developed in large grains with respect to their compositional gradients in $X_{\text {sps }}$ and $X_{\text {alm }}$ and not in the $X_{\text {grs }}$ or $X_{\text {prp }}$ gradients possibly points to uncertainties of the equilibrium Mn-Fe partitioning between phases in the thermodynamic dataset (e.g., chlorite), particularly during early growth at low$T$.

\subsubsection{Processes controlling garnet growth}

For the fast rates of metamorphic garnet crystallisation inferred from the presented analysis, chemical zoning even in the smallest garnet grains in the sample reflects growth zoning unaffected by any appreciable degree of intracrystalline diffusion. Therefore, this microstructural and chemical dataset offers the unique opportunity to analyse the observed garnet chemical zoning in light of the relative rates of the atomicscale processes that may have operated at the time of metamorphic crystallisation, and that may have controlled garnet nucleation and growth.

Rates of porphyroblast growth are commonly anticipated to be affected by the rates of long-range material transport across the rock matrix to the moving interface, and by the rates of molecular attachment and detachment at these interfaces. 
Diffusion-controlled radial growth of a spherical crystal, $d r / d t$, may be expressed as (Zener, 1949)

$$
\frac{\mathrm{d} r}{\mathrm{~d} t}=\frac{D_{i}}{\left(c_{i}^{\beta}-c_{i}^{\alpha}\right)}\left(\frac{\partial c_{i}}{\partial r}\right)
$$

where $c_{i}^{\alpha}$ and $c_{i}^{\beta}$ are the equilibrium concentrations of component $i$ at the interface of the matrix and porphyroblast, respectively, and $D_{i}$ is the diffusivity of $i$ through the matrix. In this treatment it is assumed that the differences in the molecular volumes of $\alpha$ and $\beta$ can be ignored. The compositional gradient across the depletion halo in the vicinity of the porphyroblast, $\partial c_{i} / \partial r$, controls the rate of diffusion-controlled interface movement, and decreases with an increase of porphyroblast size. This decrease in compositional gradient is related to the width of the diffusion field surrounding the porphyroblast, which is wider for larger grains compared to smaller ones. This indicates that the diffusion-controlled growth rate is expected to vary inversely with size, i.e. smaller crystals grow faster than larger ones. In this case, it may be expected that smaller, later nucleated grains that grow faster therefore contain shallower mantle gradients than large grains for a given step in $P-T$ (Fig. 2.15a), converse to the slight steepening of the compositional gradients of successively smaller grains in the studied sample.

Alternatively, there are several lines of evidence that suggest that the growth rate of garnet in 24-99 may have been controlled by the atomic attachment and detachment processes at the garnet/matrix interface: (1) approximately identical $X_{\mathrm{sps}}, X_{\mathrm{grs}}, X_{\mathrm{prp}}$, and $X_{\text {alm }}$ zoning of all crystals that fall within the same garnet size class; (2) roughly equivalent $X_{\mathrm{sps}}, X_{\mathrm{grs}}, X_{\mathrm{prp}}$, and $X_{\mathrm{alm}}$ contents in the rims of all crystals of the entire garnet population, indicative of simultaneous cessation of growth in an equilibrated matrix with respect to major end-members; and (3) garnet core $X_{\mathrm{sps}}, X_{\mathrm{grs}}, X_{\mathrm{prp}}$, and 
$X_{\text {alm }}$ contents of the entire garnet population that can be predicted well with the THERIA_G model which distributes garnet volume according to the constraints of interface-controlled growth.

The interface-controlled radial rate of growth of macroscopic grains, $d r / d t$, is given by (Christian, 2002, Balluffi et al., 2005)

$$
\frac{\mathrm{d} r}{\mathrm{~d} t}=M \Delta G_{r}
$$

where $\Delta G_{r}$ is the chemical driving force for growth and $M$ is interface mobility. According to this growth law, the radial rate of interface-controlled growth is sizeindependent, and porphyroblasts grow with the same radial rate for a given step in $P-T-X$ space, resulting in identical compositional gradients independent of the sizes and positions of the crystals in the rock (Fig. 2.15b). Such chemical zoning is not observed in the studied sample.

However, the more general expression accounting also for the influence of interface curvature on the overall Gibbs energy change during porphyroblast growth may be used to explain the chemical trends observed in garnet of 24-99. This general rate-law for interface-controlled growth reads (Christian, 2002, Balluffi et al., 2005; Gaidies et al. 2017)

$$
\frac{\mathrm{d} r}{\mathrm{~d} t}=M\left(\Delta G_{r}-\frac{2 \sigma}{R}\right)
$$

where $R$ is the radius of interface curvature and $\sigma$ is the interfacial energy. According to 2.3$)$, for relatively small crystals and reactions close to equilibrium, $\Delta G_{r}$ may approach values similar in magnitude to $\sigma$ so that the thermodynamic driving force effectively acting on the interface decreases, and the growth rate becomes sizedependent, reflecting the increase in Gibbs energy with interface curvature. Only in 
that case, and amplified by relatively large $\sigma$, small crystals will grow slower than larger ones for a given step in time and under the same $P-T-X$ conditions. In terms of porphyroblast growth, compositional gradients that develop as the interface moves would be steeper in smaller crystals compared with larger ones but would be the same for crystals of identical size if these crystals grew simultaneously (Fig. 2.15 c).

Hence, size-dependent interface-controlled growth according to expression 2.3 may explain the observed progressive steepening of compositional gradients with decreasing grain size. The successful prediction of the chemical zoning of the garnet population using the THERIA_G model may indicate relatively low chemical driving forces as expected for crystal growth close to equilibrium. Since the observed steepening in compositional gradients with decreasing grain size suggests that the slowing of garnet growth only affected progressively later nucleated crystals, it may be interpreted that $\sigma$ increased over the crystallisation interval (e.g., Gaidies et al., 2011). Such an increase may be related to the progressive loss of chlorite during garnet crystallisation in 24-99. Given that the garnet crystallisation simulations predict the garnet core compositions of the majority of garnet crystal sizes assuming negligible degrees of reaction overstep, an increase in $\sigma$ is assumed to have had only minor influence on the energy barrier to nucleation.

However it is important to recognise that the two end-member scenarios in the kinetics of porphyroblast crystallisation may not preclude each other, such that a metamorphic system close to equilibrium may alternate between diffusion- and interfacecontrolled reaction kinetics, reflecting the change of the energy barriers associated with the underlying processes as crystallisation proceeds. As proposed by Daniel \& Spear (1999), if diffusion-controlled growth did yield chemical potential gradients around growing garnet in the studied sample, they did not significantly affect the incorporated garnet compositions, such that the bulk system can be effectively mod- 
elled using an interface controlled assumption. This may well be the case for small scale fluctuations in $X_{\text {grs }}$, superimposed on broad core-to-rim zoning controlled by the evolving whole-rock chemistry.

\subsection{Conclusions}

The LHS of the Sikkim Himalaya is characterised by rapid rates of $T-t$ evolution during garnet crystallisation, in excess of $100{ }^{\circ} \mathrm{C} \mathrm{Myr}^{-1}$, and as a consequence, the smallest analysed garnet crystals within sample 24-99 have undergone negligible intracrystalline diffusion with respect to their major end-member components. The population contains a compositional record of the full history of crystallisation over $<1 \mathrm{Myr}$, from incipient nucleation at approximately $518^{\circ} \mathrm{C}$ and $4.5 \mathrm{kbar}$ to cessation of growth at approximately $565{ }^{\circ} \mathrm{C}$ and 5.6 kbar. Forward modelling suggests that between these bounds, the garnet population progressively nucleated within a changing $\mathrm{EBC}$ at a succession of near-equilibrium states with respect to the components considered in the model.

While neither interface- nor diffusion-controlled end-member models of metamorphic crystallisation may alone be adequate to account for the complexity within large natural datasets, interface-controlled crystallisation close to equilibrium in a fractionating chemical system similar to that simulated here may be considered a powerful model scenario to predict the major element garnet growth zoning. Application of such an end-member model to the garnet-grade metapelite in this study results in (1) good fits between the observed and predicted garnet crystal sizes, and (2) the prediction of the systematic compositional variation observed from core to rim of the entire garnet population. Steepening of compositional gradients with decreasing grain size can be attributed to size-dependent garnet growth due to an increase in 
the interfacial energy as the crystallisation interval proceeds.

Consideration of potential kinetic inhibitors to the attainment of chemical equilibrium should be considered in light of both our increased understanding of these departures from equilibrium, and our ever-increasing ability to constrain them (e.g., Pattison \& Tinkham, 2009; Kelly et al., 2013, Spear et al., 2014). However, the sample studied here suggests that the departure from equilibrium during prograde metapelitic garnet crystallisation may not be significant even in rapidly heated rocks, and that the assumption of equilibrium should continue to be used as an informative model for constraining metamorphic histories. Comprehensive assessment of a representative population of garnet crystals can be used to infer details of the metamorphic $P-T-t$ evolution that cannot be obtained from the study of a single garnet porphyroblast. Combined with the application of high-resolution chemical and isotopic analyses, such detailed studies of population-wide compositional variations are likely to continue to contribute to our understanding of the fundamental processes operating during metamorphism. 


\section{Tables and Figures}

Table 2.1: Bulk composition of sample 24-99, in wt. \% as determined by WDXRFA and in moles of cations, as used in Theriak-Domino and THERIA_G.

\begin{tabular}{lrrrrrrrrrrr}
\hline Oxide & $\mathrm{SiO}_{2}$ & $\mathrm{TiO}_{2}$ & $\mathrm{Al}_{2} \mathrm{O}_{3}$ & $\mathrm{Fe}_{2} \mathrm{O}_{3}$ & $\mathrm{MnO}$ & $\mathrm{MgO}$ & $\mathrm{CaO}$ & $\mathrm{Na}_{2} \mathrm{O}$ & $\mathrm{K}_{2} \mathrm{O}$ & LOI & $\sum$ \\
wt. \% & 64.85 & 0.61 & 18.73 & 6.44 & 0.05 & 1.89 & 0.55 & 0.87 & 3.60 & 2.36 & 100.06 \\
\hline Cation & $\mathrm{Si}$ & $\mathrm{Ti}$ & $\mathrm{Al}$ & $\mathrm{Fe}^{2+}$ & $\mathrm{Mn}$ & $\mathrm{Mg}$ & $\mathrm{Ca}$ & $\mathrm{Na}$ & $\mathrm{K}$ & $\mathrm{H}$ & $\mathrm{C}$ \\
mol & 63.60 & 0.05 & 12.65 & 4.76 & 0.04 & 2.77 & 0.57 & 1.65 & 4.51 & 100.00 & 100.00 \\
\hline
\end{tabular}




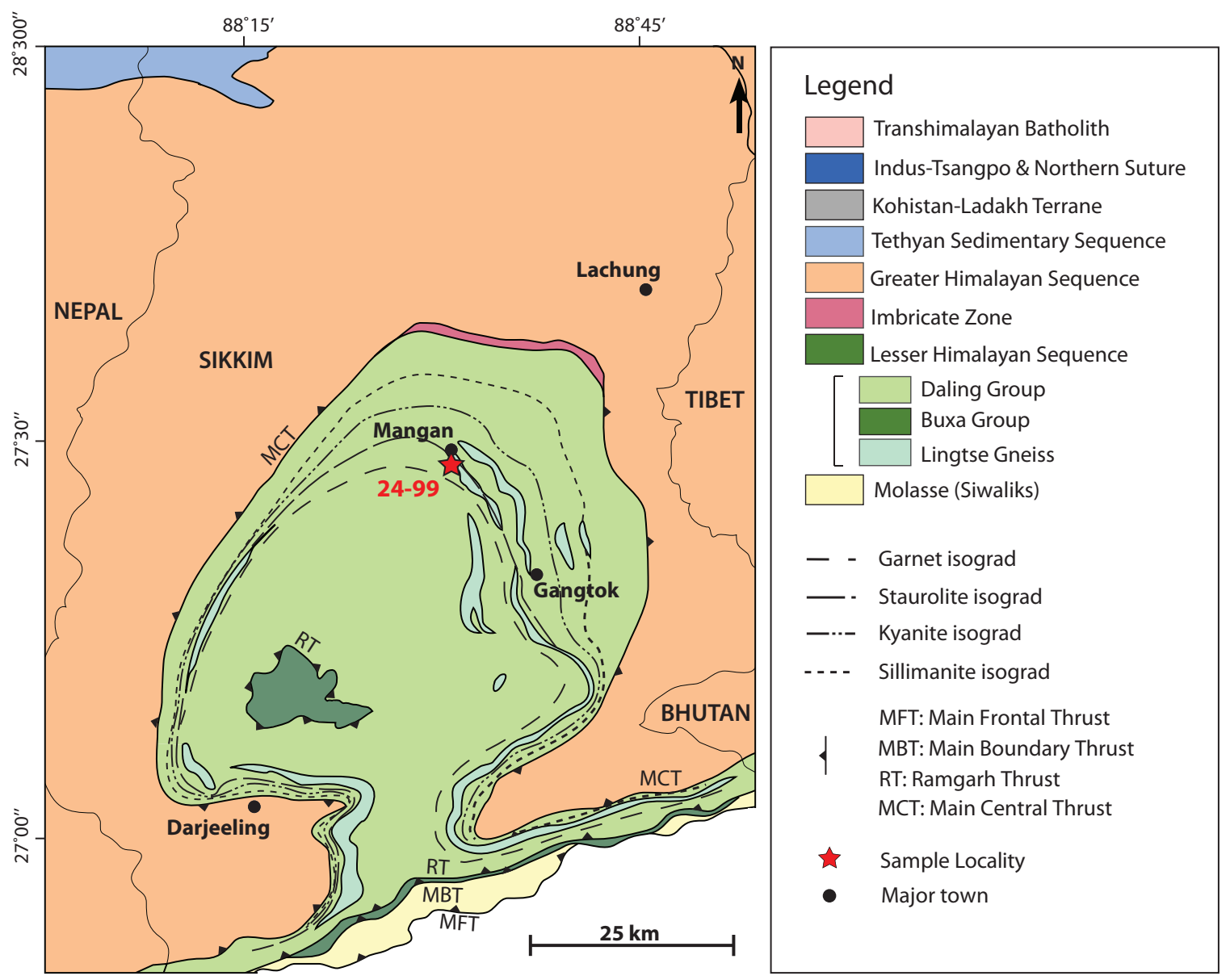

Figure 2.1: Geological map of the Sikkim Himalaya, with sample locality highlighted by a red star. Modified after Chakraborty et al. (2016). 

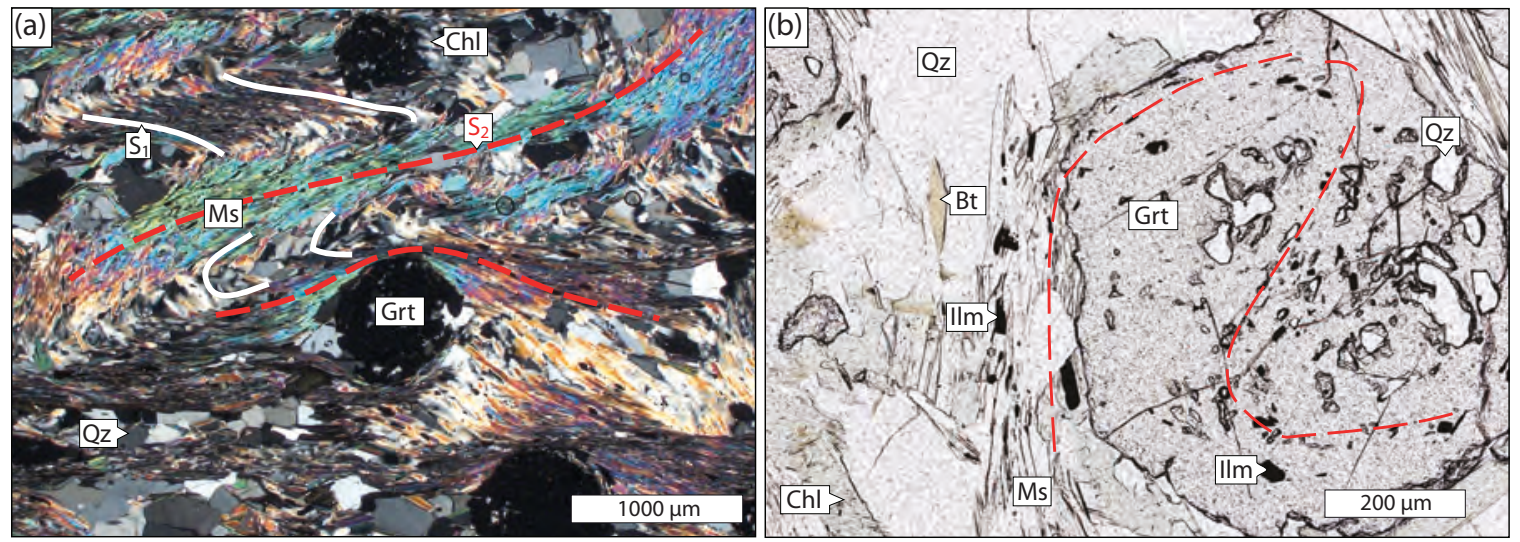

Figure 2.2: (a) Cross-polarised photomicrograph showing subhedral garnet porphyroblasts within a crenulated $\mathrm{S}_{1}$ muscovite-rich matrix foliation. (b) Plane-polarised photomicrograph of syn-kinematic euhedral-subhedral garnet porphyroblast with sigmoidal inclusion trails that are continuous with external foliation at the rims. Mineral abbreviations after Whitney \& Evans (2010). 

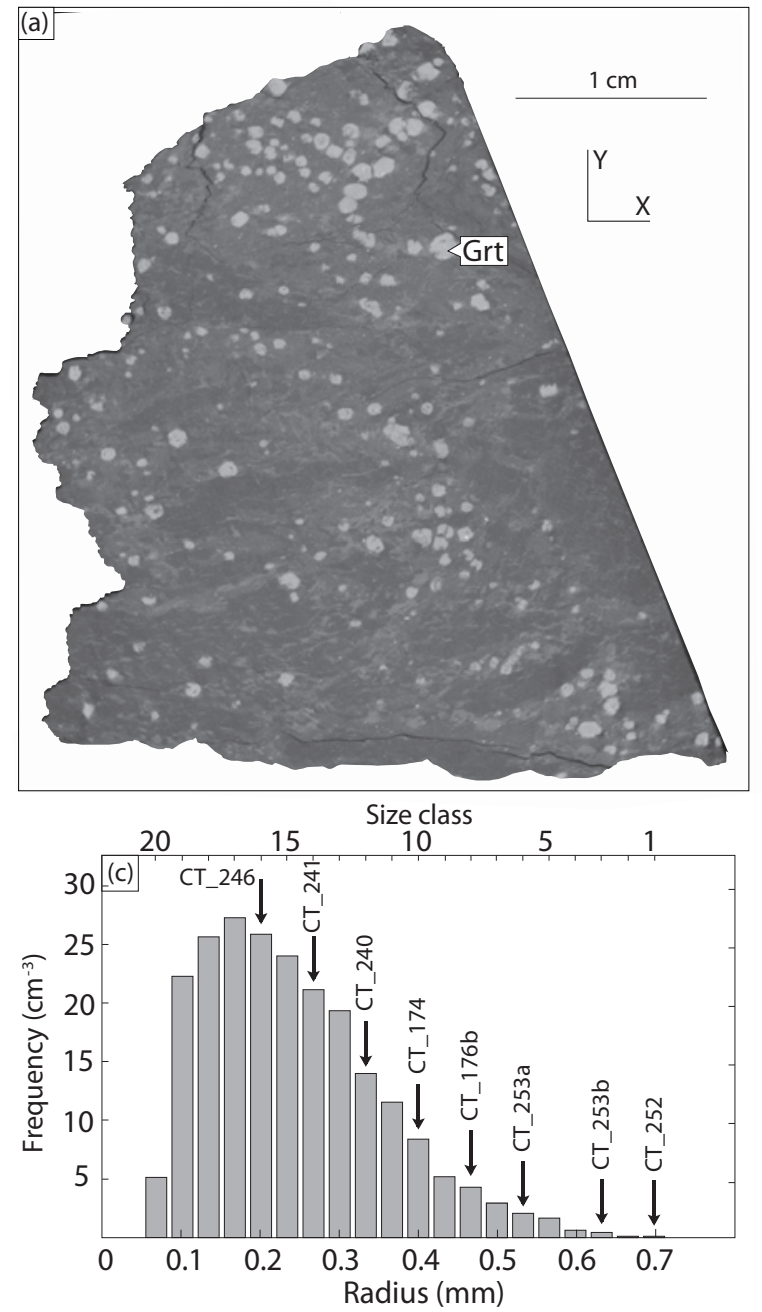

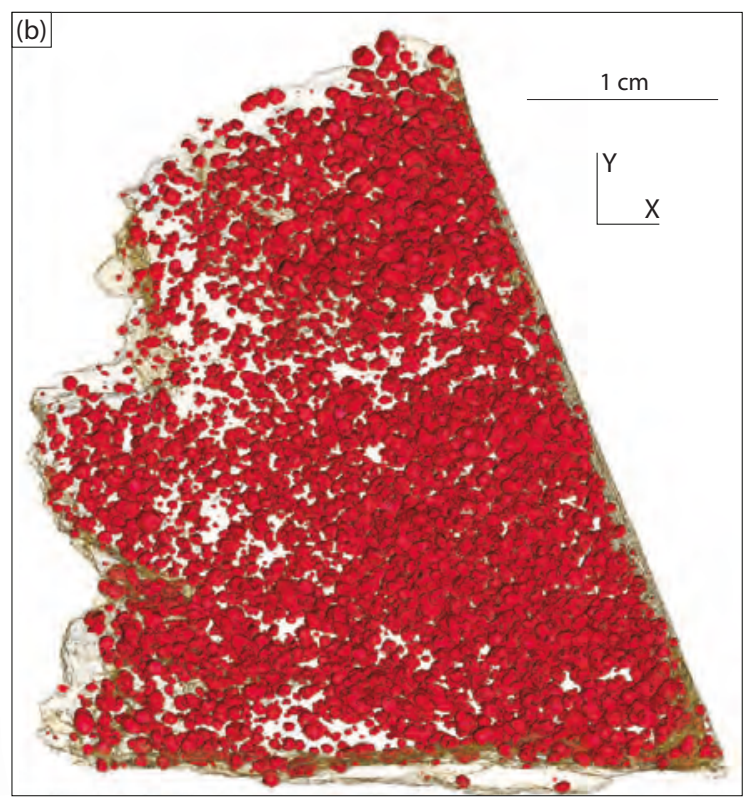

(d)

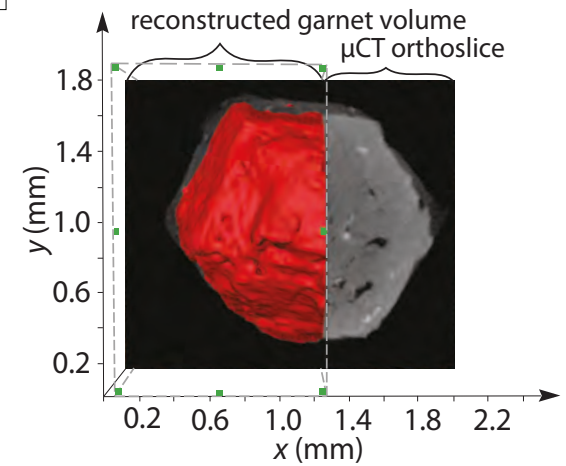

Figure 2.3: (a) X-ray attenuation image of sample 24-99. (b) Corresponding prismatic volume of 24-99 analysed by XR- $\mu \mathrm{CT}$, and X-Y view of the surface-rendered scanning volume illustrating the size, shape and spatial distribution of garnet, in red. (c) Crystal size distribution (CSD) as determined from extracted garnet volume, with 20 size classes. Black arrows highlight the size classes from which representative central sections are displayed (Figs. 2.5 and 2.4). (d) Largest garnet crystal analysed with $\mathrm{XR}-\mu \mathrm{CT}$ (size class 1 - CT252). Greyscale region displays XR- $\mu \mathrm{CT}$ central orthoslice perpendicular to the $z$-direction, while red region displays partially reconstructed $3 \mathrm{D}$ volume of this crystal. 


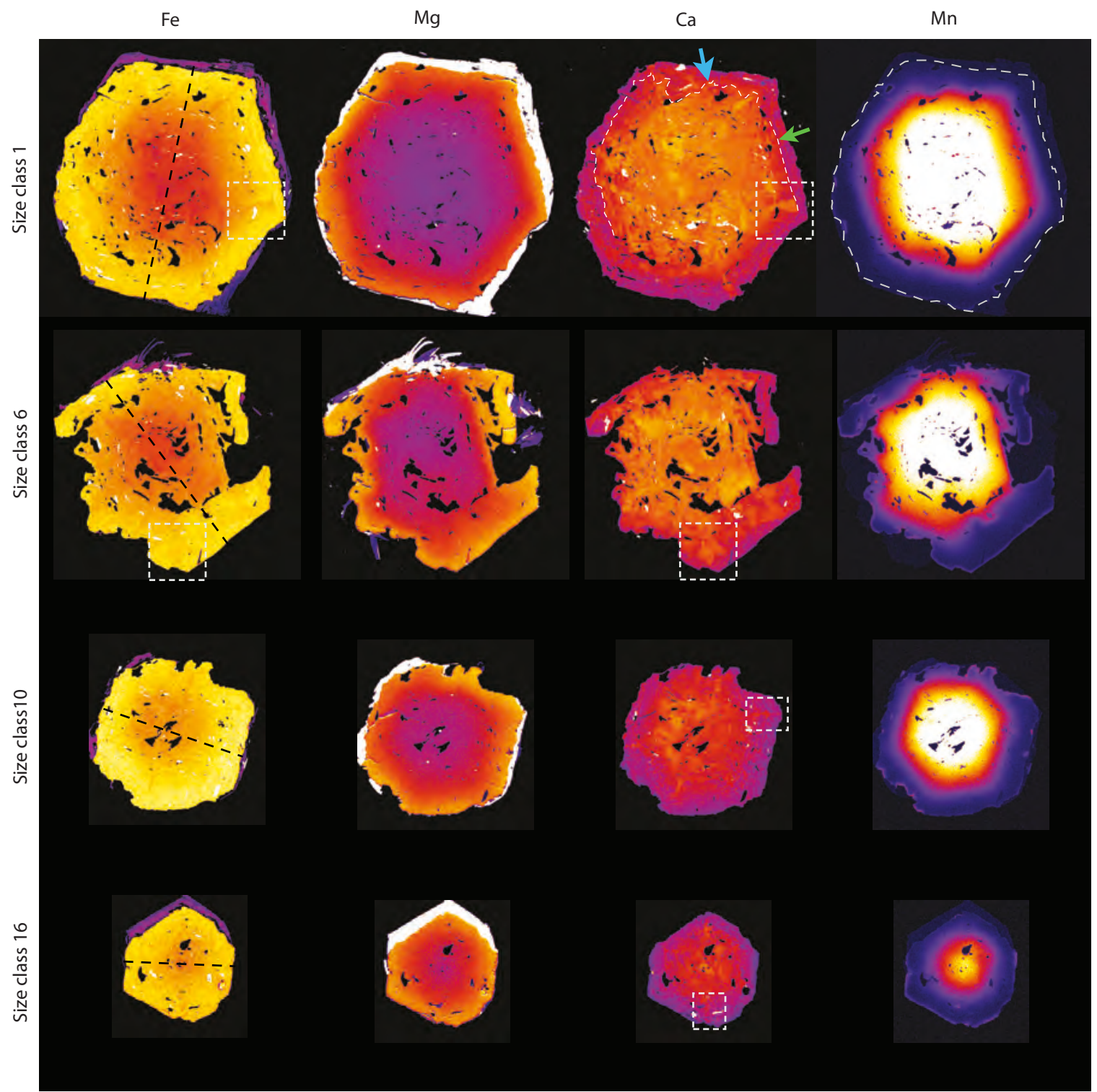

Relative count

$1 \mathrm{~mm}$

low high

Figure 2.4: X-ray maps of four major end-member cations in garnets from sample 24-99. White dashed line in CT252 Mn map outlines garnet crystal, black dashed line corresponds to the position of compositional analysis profiles in Fig. 2.5. White dotted boxes highlight sector zoning. 

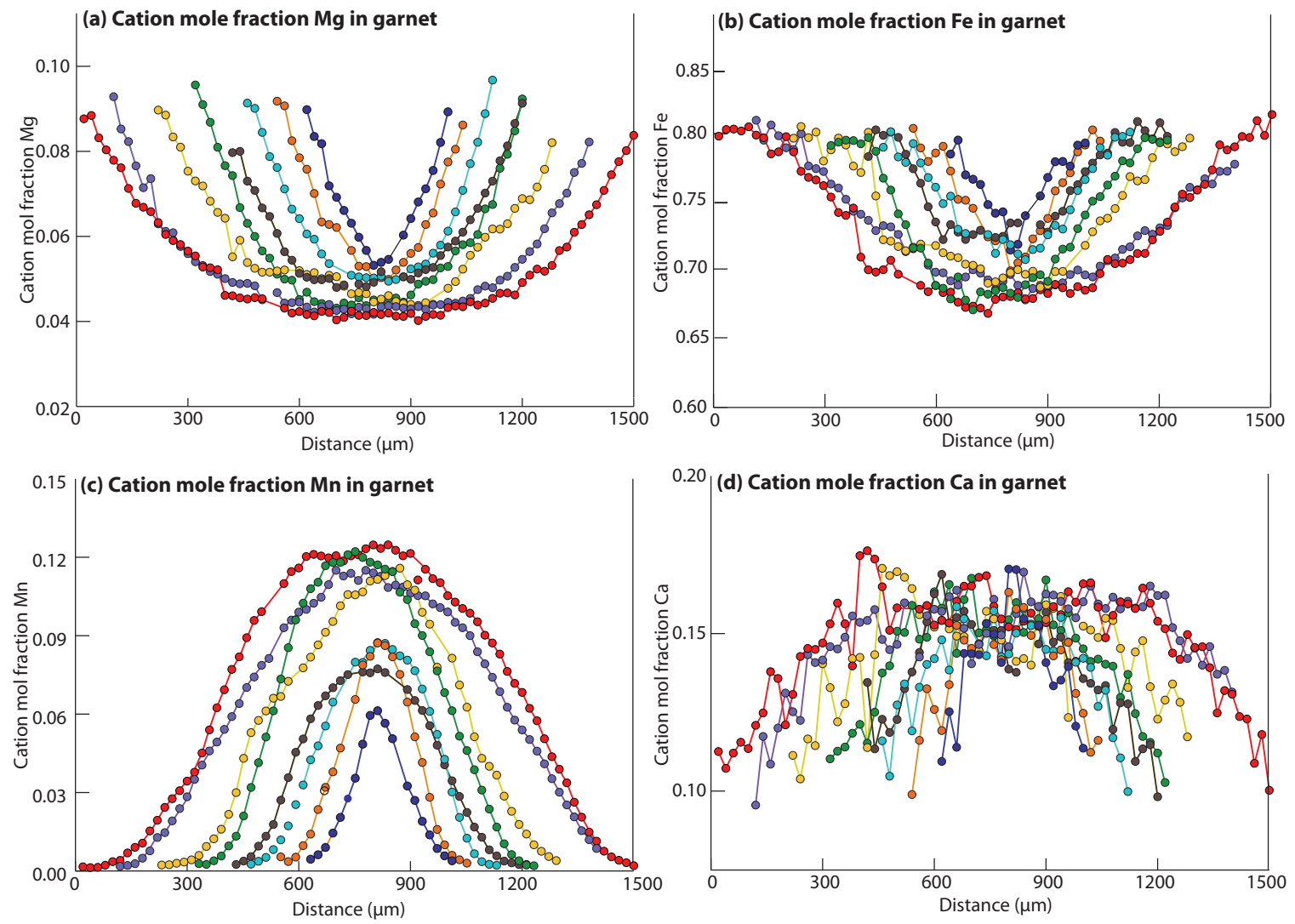

$\begin{array}{llll}\text { Size class } 1 \bullet & \text { Size class } 6 \circ & \text { Size class } 10 \bullet & \text { Size class } 14 \\ \text { Size class } 3 \bullet & \text { Size class } 8 \bullet & \text { Size class } 12 \bullet & \text { Size class } 16\end{array}$

Figure 2.5: Garnet compositional line profiles across geometric cores of grains that are representative of the sample 24-99 CSD. (a)-(d) represent cation mole fractions of $\mathrm{Mg}, \mathrm{Fe}, \mathrm{Mn}$ and $\mathrm{Ca}$ in garnet, respectively. 


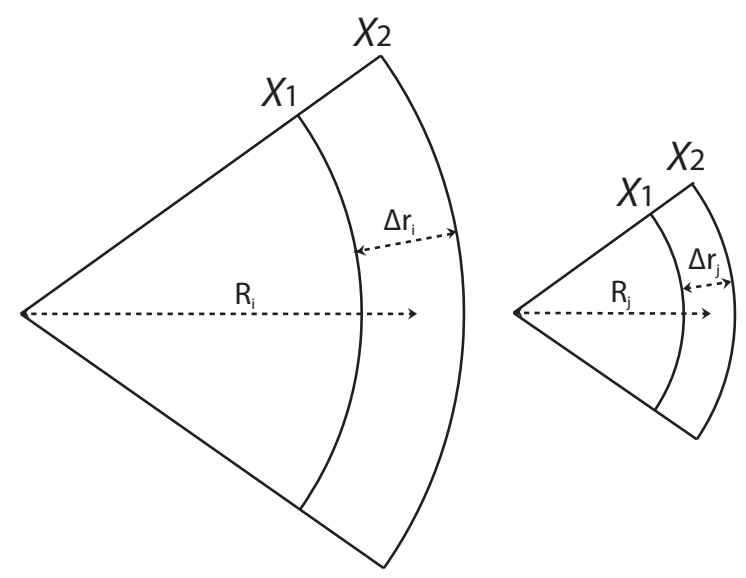

Figure 2.6: Schematic section through cores of two garnet crystals. $\Delta r_{i, j}$ is the distance between two compositional contours $X_{1}$ and $X_{2}$ in crystal $i$ and $j . \mathrm{R}_{i, j}$ is the distance from the crystal centre to the mid-point between $X_{1}$ and $X_{2}$ in each crystal.

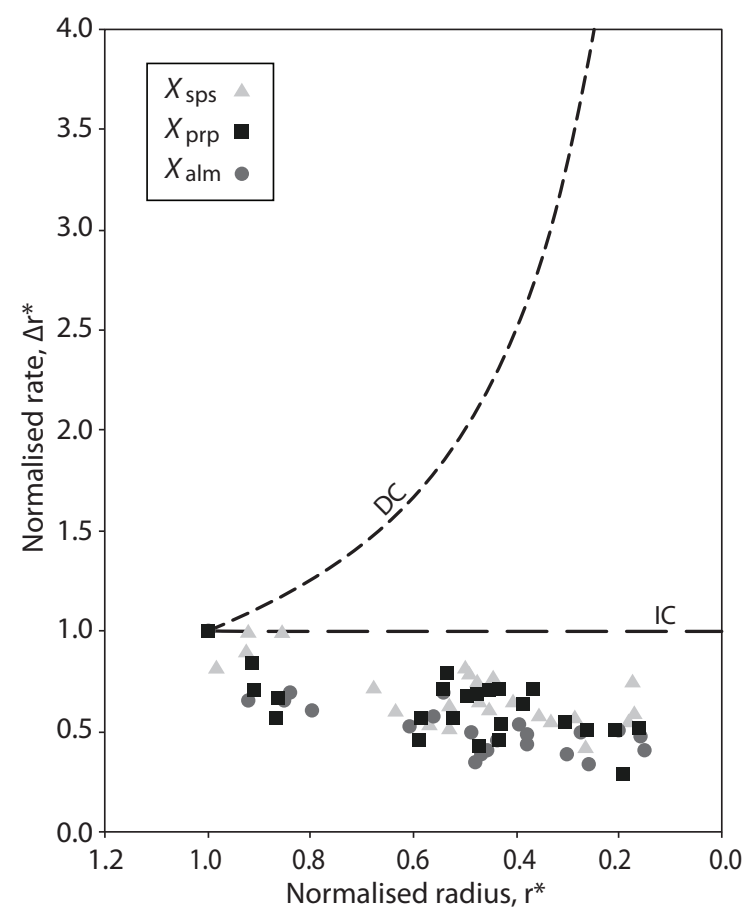

Figure 2.7: Normalised radius-rate plot for selected compositional contours of $X_{\mathrm{sps}}$ (0.02 and $0.03 \mathrm{~mol} \%), X_{\text {prp }}(0.07$ and $0.08 \mathrm{~mol} \%)$ and $X_{\text {alm }}(0.765$ and $0.775 \mathrm{~mol}$ $\%$ ). Fine and coarse dashed lines represent expected radius-rate relationships based on diffusion-controlled (DC) and interface-controlled (IC) growth, respectively. 


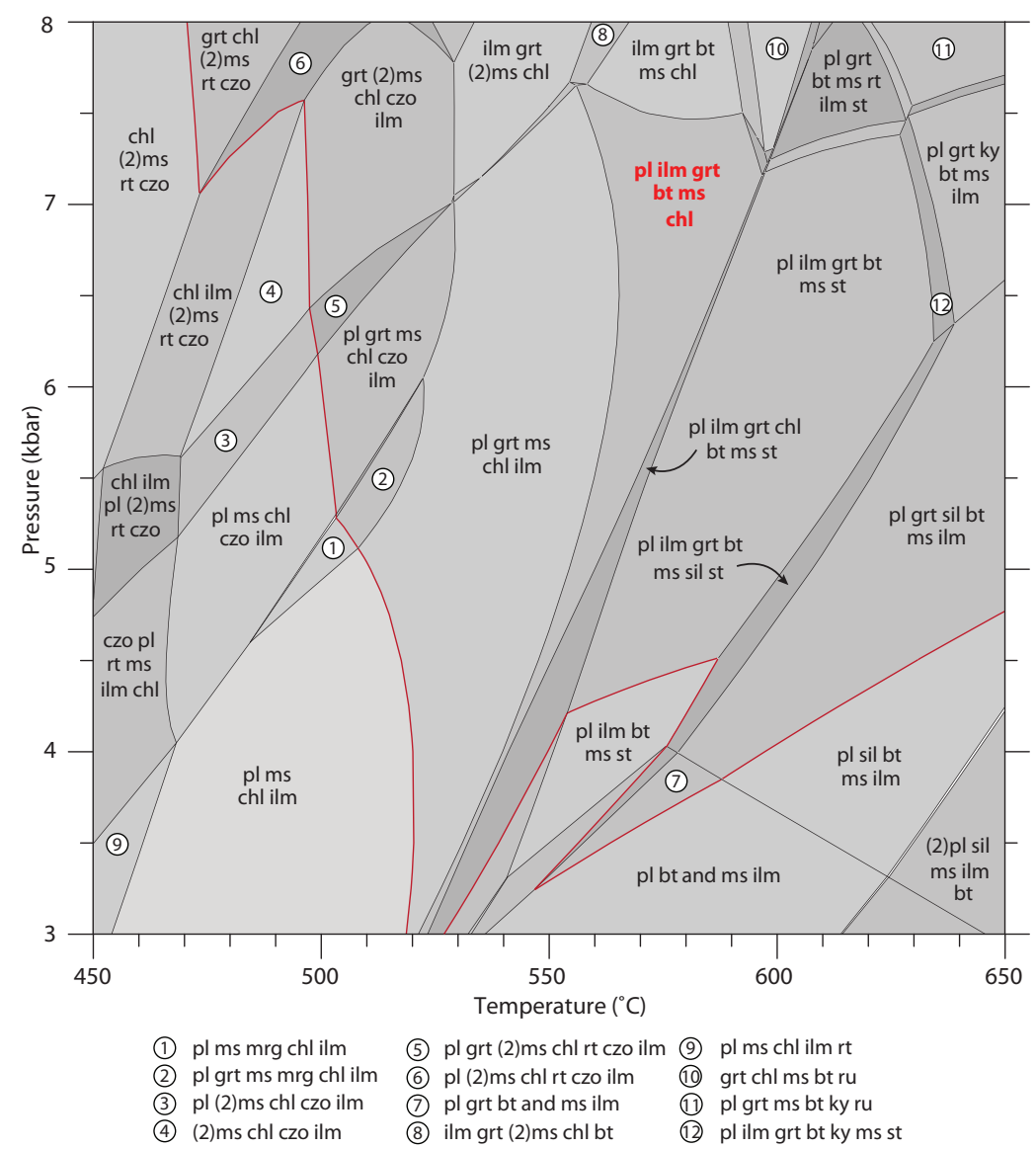

Figure 2.8: Calculated $P-T$ phase equilibria for sample 24-99 with assemblages labelled, calculated with DOMINO (de Capitani \& Petrakakis, 2010) and shaded according to variance. All assemblages contain quartz, graphite and $\mathrm{COH}$-fluid in excess. Observed metamorphic field assemblage is labelled in red and garnet-in univariant line highlighted in red. 


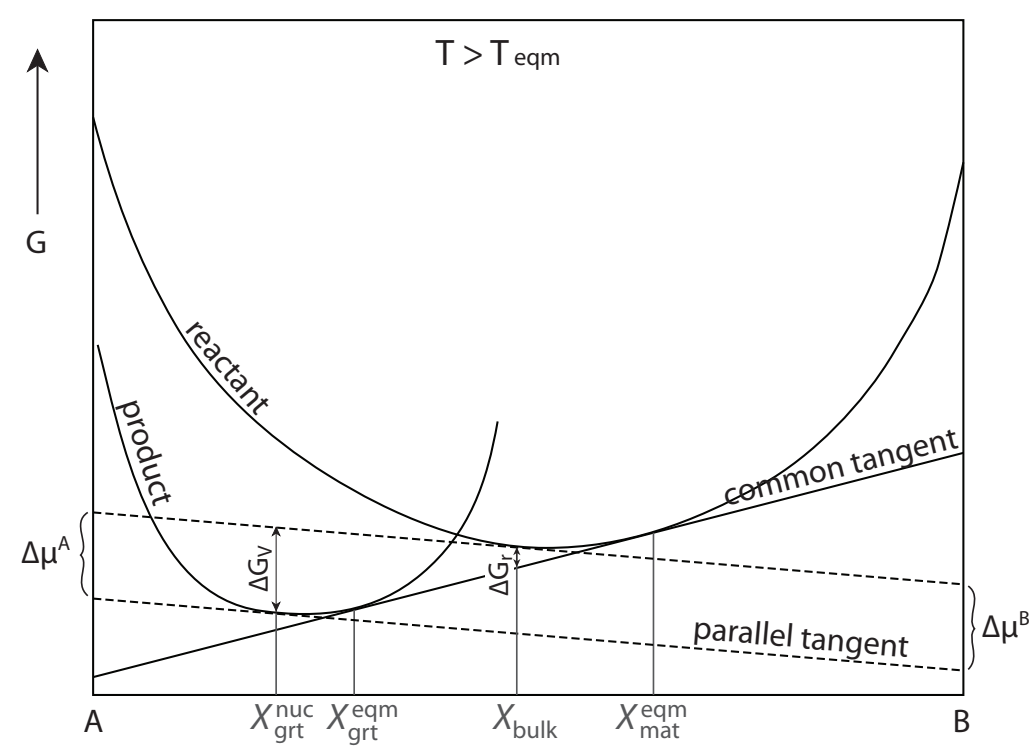

Figure 2.9: Schematic molar Gibbs energy-composition $(G-X)$ diagram for a two component system at a $T$ above the equilibrium $T$, illustrating the approach used to calculate the chemical composition of a nucleus using the parallel $\left(X_{g r t}^{n u c}\right)$ and common $\left(X_{\text {grt }}^{\text {eqm }}\right)$ tangent methods, the chemical driving force for nucleation, $\Delta G_{V}$, and the chemical driving force for the overall reaction, $\Delta G_{r}$, after some overstep of the equilibrium condition, $T_{\text {eqm }}$. $X_{\text {bulk }}$ is the bulk composition of the system. See text for explanation. Modified after Gaidies et al. (2011).

Figure 2.10 (following page): (a)-(d) Chemical composition of a garnet nucleus using the common tangent method ( $X_{\text {grt }}^{e q m}$, solid contours), and the parallel tangent method ( $X_{\text {grt }}^{n u c}$, colour-coded) for the $P-T$ conditions of interest. (e) Isopleth intersection for $X_{\text {grt }}^{e q m}$ (small circle), $X_{\text {grt }}^{\text {nuc }}$ (large circle) at $\sim 519{ }^{\circ} \mathrm{C} / 4.4 \mathrm{kbar}$ and $\sim 527{ }^{\circ} \mathrm{C} / 4.8$ kbar, respectively. Solid lines reflect $X_{\text {grt }}^{\text {eqm }}$, dashed lines reflect $X_{\text {grt }}^{n u c}$. (f) Driving force for garnet nucleation, $\Delta G_{V}$. 

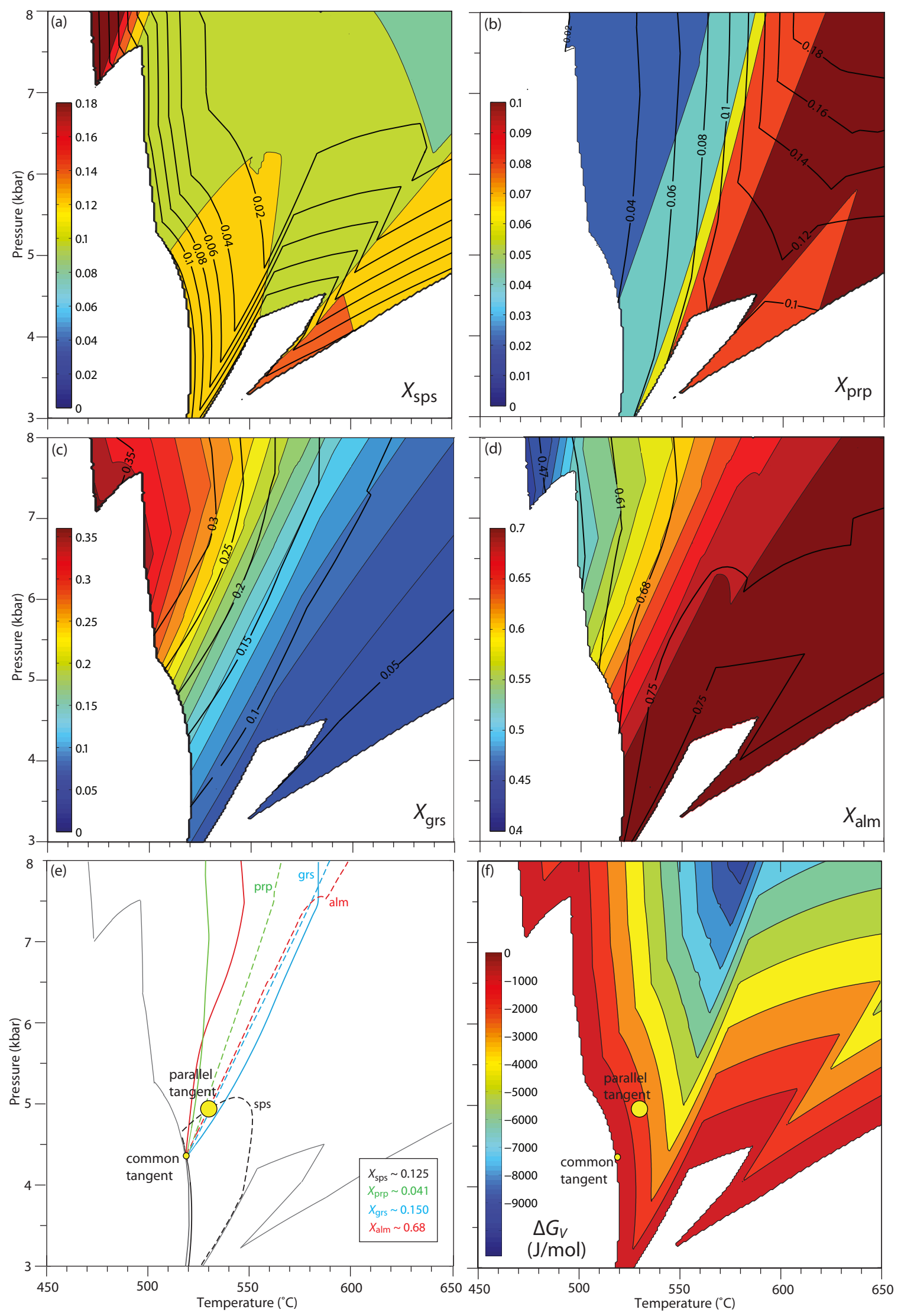
Figure 2.11 (following page): Schematic outlining the addition of concentric shells in the THERIA_G model, for four CSD classes along the $P-T$ path illustrated in (b). A given colour shade represents a single composition, distributed on the surfaces of all crystals with a thickness $\Delta s, x$ is the width of the CSD class. Note irregular time spacing of sequential schematics, and fractionating bulk matrix composition. See text for details. 

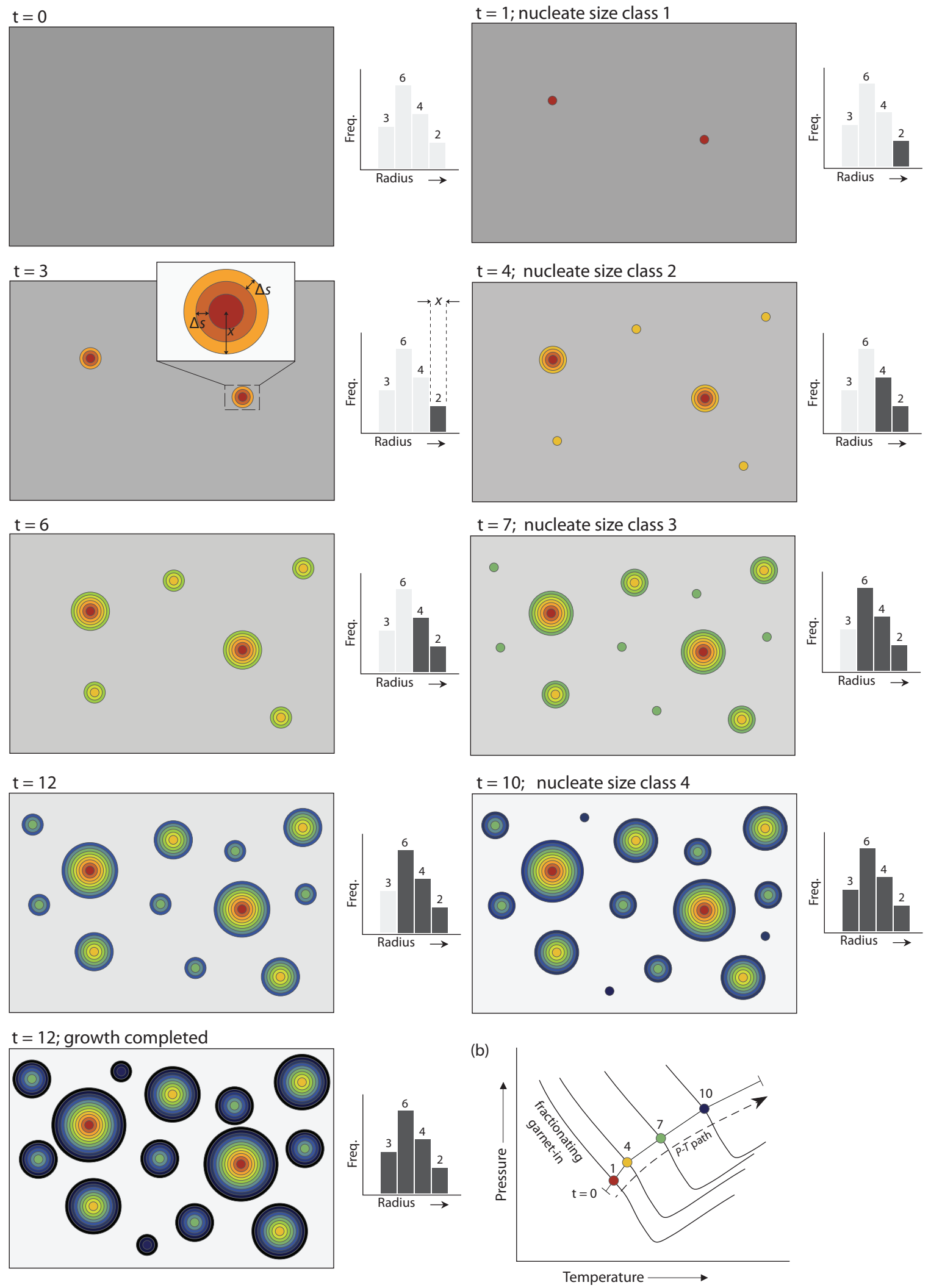

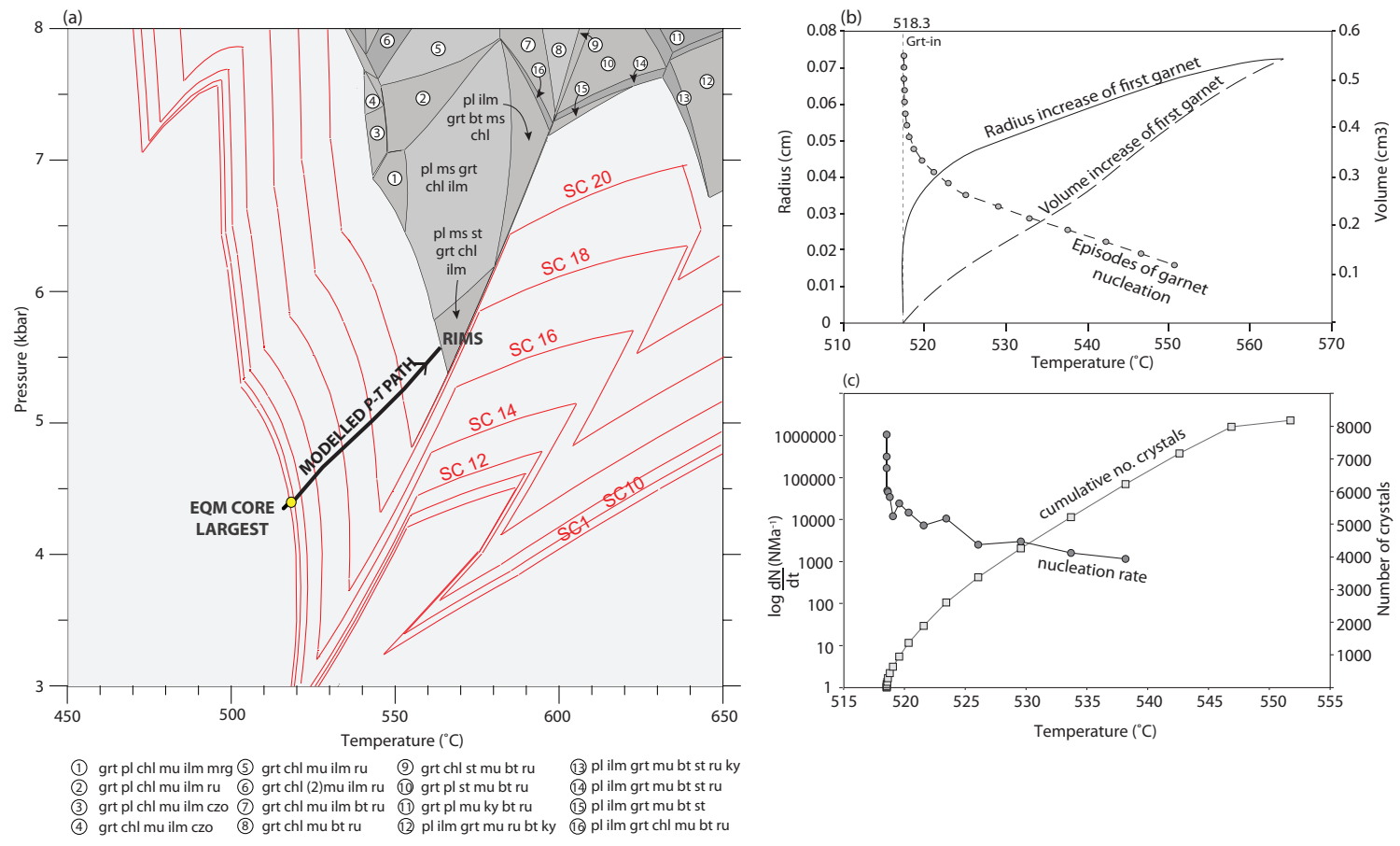

Figure 2.12: (a) Evolving garnet-in curve as fractionation of the EBC proceeds along $P-T$ path, with progressive crystallisation and nucleation of new garnet size classes (SC). Changing red garnet-in calculated using EBC at time of size class nucleation. Final phase diagram calculated with EBC at peak pressure conditions. (b) Modelled increase in radius of oldest garnet crystal with respect to progression along the $P-T$ path (solid line). Circles along dashed line are the temperatures at which each size class nucleates. (c) Modelled nucleation rate and simulated accumulation of crystal abundance, normalised to true number of crystals in 24-99.

Figure 2.13 (following page): Results of THERIA_G modelling for garnet compositional profiles displayed in Fig. 2.5, with crystal size decreasing down-column. Dotted lines represent the temperature of incipient garnet growth for that size class. 

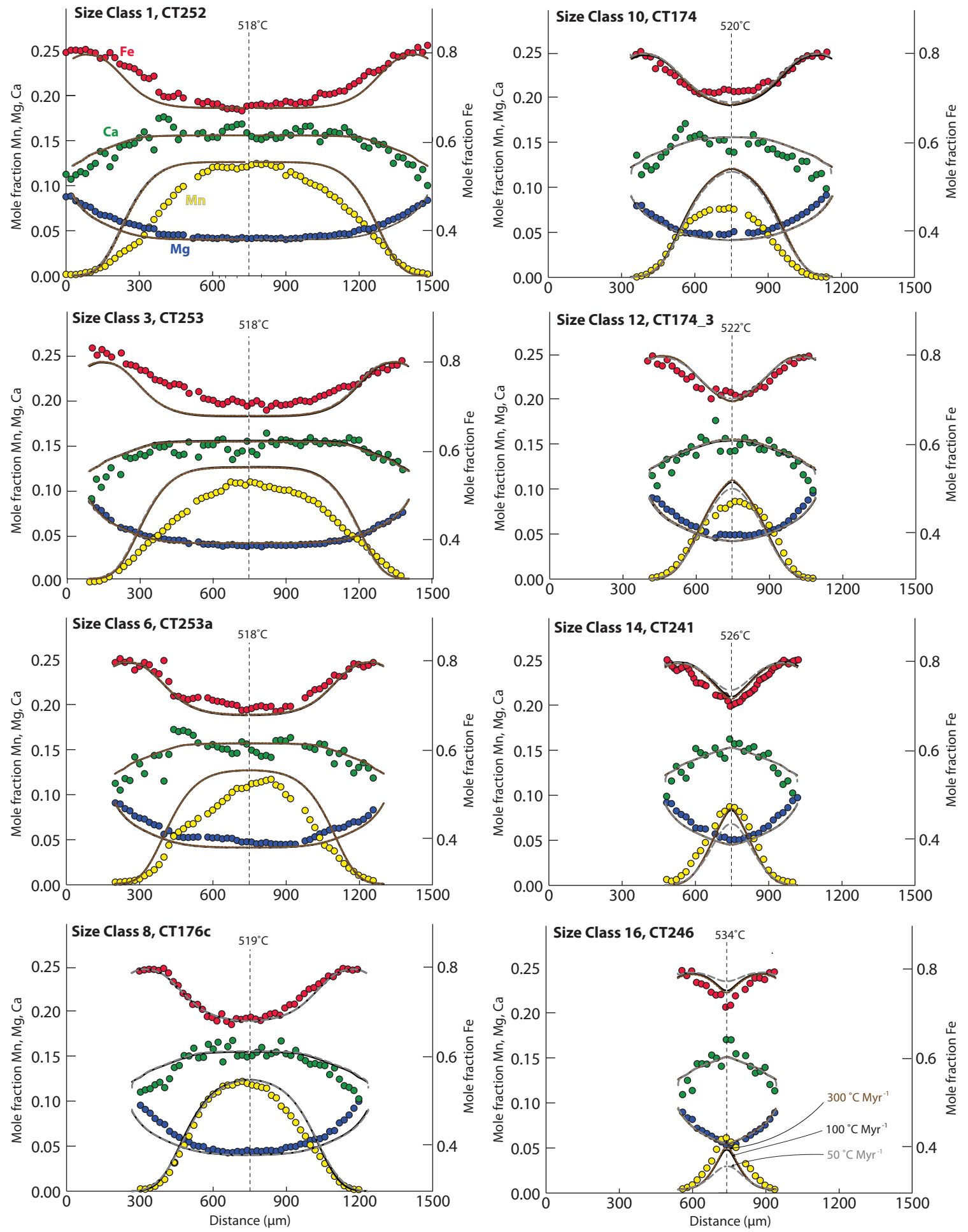


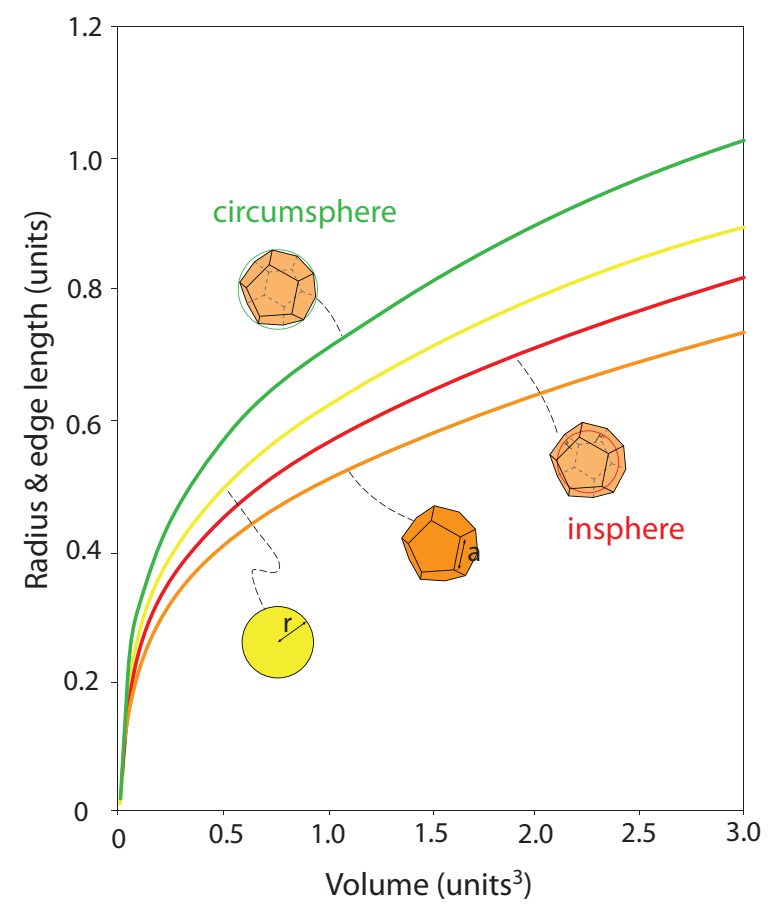

Figure 2.14: Rate of change of radius and edge-length with volume for spheres and dodecahedra. Yellow line represents sphere radius, $r$, increase, and orange is dodecahedra edge length, a. Green and red curves show increase in radius of dodecahedral circumsphere and inspheres, respectively.
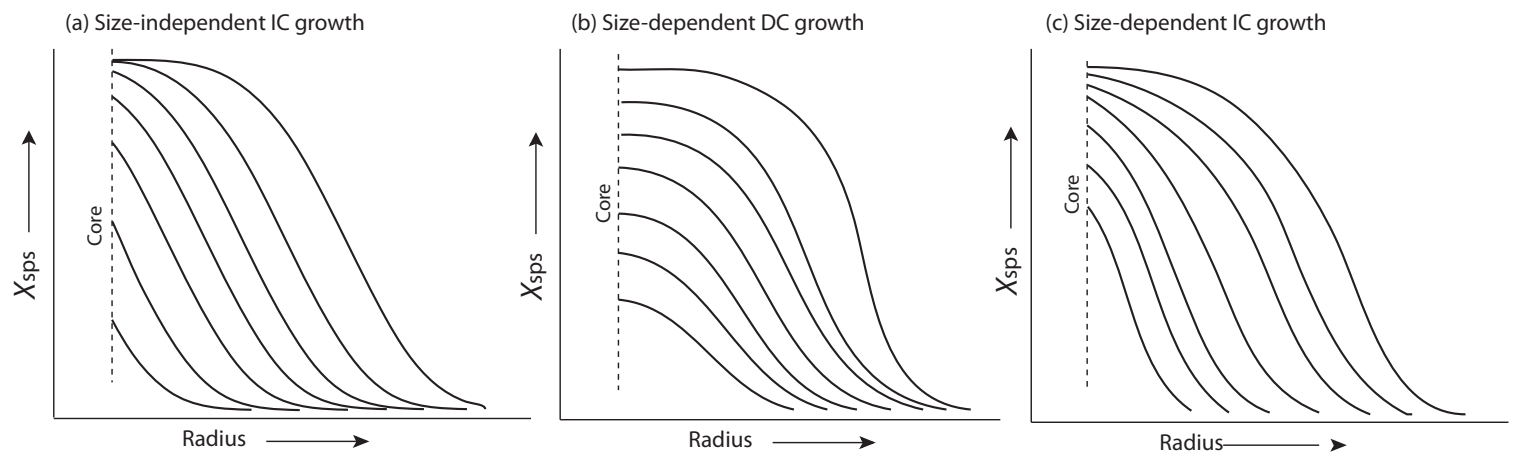

Figure 2.15: Schematic compositional zoning profiles anticipated for growth according to different kinetic scenarios. (a) size-independent interface-controlled growth; (b) size-independent diffusion-controlled growth; and (c) size-dependent interfacecontrolled growth. See text for details. 


\section{Chapter 3}

\section{Trace element zonation of a garnet population: length scales of equilibration and deformation during crystallisation}

\subsection{Introduction}

Metamorphic garnet is an invaluable tool in a petrologists arsenal, largely because of its chemical sensitivity to changing pressure and temperature $(P-T)$ conditions over the duration of garnet crystallisation. The ability to reconstruct changing $P-T$ by coupling the study of major element mineral chemistry and the principals of equilibrium thermodynamics has yielded great insight into both regional tectonic processes (e.g., Spear et al. 1984; Zhao et al., 2000; Catlos et al., 2001, Dasgupta et al., 2004), and our understanding of the grain-scale controls on metamorphic mineral crystallisation (e.g., Chernoff \& Carlson, 1997, Ague \& Carlson, 2013, George \& Gaidies, 2017).

While major element zonation developed in a garnet crystal during its growth continues to be informative, trace elements, particularly the rare-earth elements (REE), are generally thought to have order of magnitude slower diffusivities in both garnet and in the matrix compared to major elements (e.g., Cherniak, 2005; Carlson, 2012). 
Consequently, they are less susceptible to diffusional homogenisation and may record a more primary geochemical signature of garnet crystallisation not otherwise preserved by major elements. Owing in part to developments in laser ablation-inductively coupled-mass spectrometry (LA-ICP-MS) analytical capabilities and detection limits (e.g., Jackson et al., 1992; Norman et al., 1996; Heinrich et al., 2003; Woodhead et al., 2007; Ulrich et al., 2009; Paul et al., 2012), trace element zonation within garnet has been shown to yield information pertaining to a range of metamorphic processes. For example, REE zoning has been used to shed light on accessory mineral reaction histories (e.g., Lanzirotti, 1995, Pyle \& Spear, 1999, Yang \& Pattison, 2006), changes in the major phase assemblage over the duration of garnet crystallisation (e.g., Konrad-Schmolke et al., 2008), garnet resorption histories (Pyle \& Spear, 1999 Kohn \& Malloy, 2004), metamorphic fluid infiltration, and diffusion-limited uptake and trace element equilibration (Skora et al., 2006; Moore et al., 2013; Raimondo et al. 2017).

The primary incorporation and spatial distribution of trace elements in garnet has also been shown to have critical importance to $\mathrm{Lu}-\mathrm{Hf}$ and $\mathrm{Sm}-\mathrm{Nd}$ garnet ages, and on their tectonometamorphic implications (Skora et al., 2006, 2009; Kohn, 2009, Kelly et al., 2011). While Anczkiewicz et al. (2012) and Smit et al. (2013) effectively assessed trace elements along line profiles in a range of garnet sizes, most studies pertain to the analysis of a single crystal. The two dimensional (2D) distribution of trace elements in garnets of different sizes has the potential to yield information that improves our understanding of nucleation and growth mechanisms over the duration of metamorphic crystallisation. Additionally, the trace element distribution in garnet may yield information about their transport and distribution in the intergranular medium during this interval.

This study investigates the distribution of trace elements in metamorphic garnets 
that are representative of a rapidly crystallised garnet population in a metapelite from the Sikkim Himalaya. A LA-ICP-MS raster mapping technique is applied to reveal the complex and highly variable trace element patterns in this centrally sectioned population. These data, considered in conjunction with results of major element diffusion and thermobarometric modelling in George \& Gaidies (2017), suggest a lack of equilibration of the intergranular medium with respect to the REE during garnet crystallisation. However a characteristic change in the REE distribution observed in each garnet of this study suggests that there was a change in the diffusive lengthscale of HREE+Y during crystallisation. Elements that are strongly partitioned in matrix phases remain immobile over the duration of garnet crystallisation, and have been used to provide direct estimates of the strain rates experienced by the sample during Barrovian metamorphism.

\subsection{Regional context and sample description}

The Sikkim Himalaya, located in north-east India, comprises a well-documented 15 km-wide inverted Barrovian metamorphic sequence with increasing grade towards the Main Central Thrust (MCT) zone (Mohan et al., 1989, Dasgupta et al., 2009; Mottram et al., 2014b). The sequence is particularly well-developed in the Paleoproterozoic Daling Group of the Lesser Himalayan Sequence (LHS), in which peak metamorphic conditions of each Barrovian zone are interpreted to have been achieved approximately simultaneously at 13-10 Ma, after c. 6 Myr of Barrovian metamorphism (Catlos et al., 2004; Anczkiewicz et al., 2014).

The garnet-grade sample (sample 24-99) from the existing studies of Dasgupta et al. (2009), Anczkiewicz et al. (2014), Gaidies et al. (2015) and George \& Gaidies (2017) was selected for further characterisation using 2D LA-ICP-MS raster mapping. 
The garnets in sample 24-99 are contained within a relatively well-foliated matrix comprising muscovite, primary chlorite, biotite, quartz and plagioclase, with accessory ilmenite, tourmaline, allanite, apatite and zircon. A full petrographic description of 24-99 is provided in George \& Gaidies (2017), and only relevant details pertaining to the garnet population are duplicated here.

High-resolution X-ray micro-computed tomography (XR $\mu$-CT) scanning of a rock volume of ca. $37 \mathrm{~cm}^{3}$ shows that the garnet population comprises 8173 crystals that exhibit a log-normal crystal size distribution (CSD) with a skewness of 0.8 (Fig. 3.1 $)$. Maximum and minimum grain radii are $0.71 \mathrm{~mm}$ and $0.053 \mathrm{~mm}$ respectively, and the mean garnet radius is $0.24 \mathrm{~mm}$. Many crystals exhibit faceted surfaces, with minor retrogression to ragged chlorite along the rims of some crystals. Garnets contain curved to sigmoidal inclusion trails comprising aligned ilmenite, apatite, allanite and flattened quartz that are commonly continuous with the external foliation. No xenotime or monazite has been documented in the sample, neither in the matrix nor as inclusions in garnet.

Electron microprobe analysis (EPMA) on central-sections of numerous differently sized garnets representative of the CSD indicate that the major element compositional zoning of the population is largely concentric about the core and parallel to dodecahedral $\{011\}$ planes (Figs. $3.1 \mathrm{~b}$ and 1c). Mn exhibits typical prograde bellshaped zoning, Mg and Fe are characterised by core-to-rim increases, and Ca content decreases broadly from core-to-rim. Ca zoning is dominantly controlled by broad core-to-rim partitioning along the $P-T$ path, but patchy, short wavelength fluctuations in $\mathrm{Ca}$ (Fig. 3.1k) attest to its slower diffusivity in the rock matrix and greater affinity for maintaining disequilibrium (e.g., Chernoff \& Carlson, 1997). The population demonstrates a systematic change in core compositions from large to small crystals, and has been shown to be consistent with a model where major element 
equilibration is attained between garnet rims and the rock matrix during garnet crystallisation (Gaidies et al., 2008). The crystal size evolution of the population is in line with size-dependent interface-controlled growth kinetics (George \& Gaidies, 2017).

According to this model, progressive nucleation and growth of the garnet population occurred between $\sim 520^{\circ} \mathrm{C}, 4.5 \mathrm{kbar}$ and $\sim 565^{\circ} \mathrm{C}, 5.5 \mathrm{kbar}$ (George \& Gaidies, 2017). As shown in Fig. 3.1b, major element zoning of all garnets can be approximately predicted along this $P-T$ path for metamorphic heating rates in excess of $100{ }^{\circ} \mathrm{C} \mathrm{Myr}^{-1}$. Larger crystals have been shown to have nucleated early and small crystals nucleated later in the crystallisation history, with rapid successive nucleation of size classes $1-8$ at approximately $520{ }^{\circ} \mathrm{C}$. The model and radius-rate calculations suggest that large garnets have faster average radial growth rates than small garnets, with crystallisation of the whole population over $<1$ Myr. The preservation of prograde growth zoning of $\mathrm{Mn}$ in the smallest crystals of the population, and the presence of compositional sector zoning in Fe and Ca along $\{211\}$ crystal faces (Fig. 3.1.) similarly attests to a rapid average crystallisation history. Such rapid prograde evolution of the LHS is consistent with constraints from Anczkiewicz et al. (2014) and Mukhopadhyay et al. (2017), and is potentially related to elevated average radiogenic heat production in rocks from the Himalaya (Faccenda et al., 2008) or shear heating in the vicinity of the MCT zone (e.g., Le Fort, 1975, Burg \& Chen, 1984, Mottram et al., 2014b), or both.

As a consequence of these rapid rates of metamorphic heating and resultant garnet growth at relatively low- $T$, the major elements in garnets of sample 24-99 have experienced negligible diffusional homogenisation during and subsequent to their growth. Thus, given the relatively simple prograde metamorphic history of the sample, investigation of the distribution of trace elements in garnet, coupled with the threedimensional (3D) analysis of their size and spatial distribution may yield nuanced 
insight into the controls on trace element incorporation into metamorphic garnet and the extent of matrix equilibration during low- to mid-amphibolite facies regional metamorphism for a range of elements.

\subsection{Methods}

\subsubsection{XR $\mu$-CT}

Sample 24-99 was imaged using the SkyScan 1173 high-resolution XR $\mu$-CT facility at Carleton University, Ottawa, in order to spatially constrain the garnet distribution within the sample. Whole-rock scanning was conducted using a $130 \mathrm{kV} / 61 \mu \mathrm{A}$ X-ray source and a $0.25 \mathrm{~mm}$-thick brass filter, with an exposure time of $1300 \mathrm{~ms}$. The sample was subsequently fragmented using the SelFrag disaggregation facility at Queen's University, Kingston, and garnets representative of the CSD were picked and mounted into grain mounts. These mounts were then each scanned at the XR $\mu$-CT facility with a $70 \mathrm{kV} / 114 \mu \mathrm{A}$ X-ray source, a $1300 \mathrm{~ms}$ exposure time and a $0.25 \mathrm{~mm}$-thick aluminium filter, in order to permit accurate central sectioning of individual crystals.

SkyScan NRecon and Aviso software was used to render each XR $\mu$-CT dataset into a 3D model, from which material statistics for the in situ and fragmented garnets were obtained. Comparison of geometric crystal-shape parameters between the two datasets allowed the identification of the spatial location of mounted garnets in the whole-rock dataset. Notably, the three large garnets analysed with LA-ICP-MS were in very close proximity in the scanned rock volume: $2.9 \mathrm{~mm}$ separates the geometric cores of crystals CT252 and CT253, and $4.6 \mathrm{~mm}$ separates crystals CT252 and CT262 (Fig. 3.2. CT252 and CT262 are situated in a common foliation plane, whereas CT253 is located in a parallel foliation plane approximately $3 \mathrm{~mm}$ away. Smaller analysed crystals could not be located in the whole rock dataset given their abundance 
in sample 24-99.

\subsubsection{Field-emission EPMA}

Compositional maps of $\mathrm{Y}$ in garnet were obtained using a Cameca SX-5 electron microprobe equipped with a field-emission gun at the University of Vienna Department of Earth Sciences. Maps were produced using a focused acceleration voltage of $20 \mathrm{kV}$, a beam current of $100 \mathrm{nA}$, and a dwell time of $300 \mathrm{~ms}$. Major element compositional maps (Fig. 3.1 c) and Y maps both have a spatial resolution of $3 \mu \mathrm{m}$.

\subsubsection{LA-ICP-MS}

LA-ICP-MS trace element maps were acquired using an ASI Resolution M-50 193 nm eximer laser ablation system housed at the University of New Brunswick Department of Earth Sciences. Ablation was conducted in a Laurin Technic Pty S-155 two-volume sample cell synchronised to an Agilent 7700x quadropole inductively coupled plasma mass-spectrometer equipped with dual external rotary pumps.

Mapping was performed on six centrally sectioned garnet crystals (from CSD classes indicated in Fig. 3.1 a), and was achieved by ablating a series of parallel rasters across the sample surface within a polygon defined by the garnet edges. Raster scans used a $17 \mu \mathrm{m}$ crater with a $5 \mu \mathrm{m} \mathrm{s}^{-1}$ stage scan speed, a laser pulse rate of $10 \mathrm{~Hz}$ and a $\sim 3 \mathrm{~J} \mathrm{~cm}^{-2}$ laser fluence. Ablation was performed in a mixed $\mathrm{He}\left(300 \mathrm{~mL} \mathrm{~min}^{-1}\right)$ and

$\operatorname{Ar}\left(930 \mathrm{~mL} \mathrm{~min}^{-1}\right)$ carrier gas, which was mixed downstream with $2 \mathrm{~mL} \mathrm{~min}^{-1} \mathrm{~N}_{2}$ to enhance sensitivity. A dual rotary pump was used in order to double the spectrometer sensitivity to medium and heavy elements. Data acquisition was completed in a time-resolved analysis mode as a single continuous experiment for each garnet. Each experiment collected a suite of 34 elements (31 trace elements plus $\mathrm{Si}$, Ca and Mn), a 15 second gas blank was run before each ablation line, and standards were measured 
approximately every 30 minutes. NIST610 was used as an external standard, with additional standards NIST612 and USGS BCR2G (Pearce et al., 1997; Rocholl, 1998).

Post-acquisition integrated scan lines were then generated offline using the internally standardised trace element data reduction scheme included in the Iolite $3^{\mathrm{TM}}$ software package (Hellstrom et al., 2008; Paton et al., 2011), an add-in for Wavemetrics Igor Pro v.6.32 ${ }^{\mathrm{TM}}$. Instrument drift and mass bias were corrected by applying a smoothed spline (spline smooth 2 in this study) along standards, and the average background was subtracted from each raster. Quantification of laser data was completed using an assumed stoichiometric value for $\mathrm{Al}$ in garnet (10.84 wt\%), with accuracy of the analysis confirmed using NIST612 and USGS BCR2G. Trace element maps were subsequently visualised as fully-quantified ppm data using the CellSpace Image function in Iolite.

The trace element content was evaluated in relevant matrix phases using single spot LA-ICP-MS analysis. Spots utilised a $33 \mu \mathrm{m}$ beam diameter, at $3 \mathrm{~Hz}$ using a $1.5 \mathrm{~J} \mathrm{~cm}^{-1}$ laser fluence. Ablation was continuous for 40 seconds, with 30 seconds gas background. Carrier gases were as previously described.

\subsection{Results}

\subsubsection{Garnet chemical composition}

From the suite of 31 trace elements collected for each of the six mapped garnet crystals, 24 of the most informative elements for CT252, CT253 and CT256 (the three largest garnets) are presented in Figs. 3.3, 3.4 and 3.5, respectively. Results from the smaller mapped crystals (CT176, CT241 and CT246) are available in Figs. 3.6, 3.7 and 3.8. Quantitative line profiles that best highlight the REE+Y zoning are shown in Figs. $3.9 \mathrm{~b}-\mathrm{g}$. Note that the locations of these profiles do not correspond 
with major element lines in Fig. 3.1p.

\section{Heavy rare-earth elements \& Y}

Compositional zoning patterns of the HREE (Dy to Lu) and Y are decoupled from the smooth, relatively continuous zoning exhibited by the major elements in garnets from sample 24-99 (Figs. 3.1b and c). For ease of interpretation, major HREE+Y zoning features for each crystal are highlighted in Fig. 3.9a.

Garnets of all sizes exhibit a patchy-to-sigmoidal distribution of HREE+Y in their core regions, (Figs. 3.3 to 3.9 and Fig. 3.10 ). At the chemical core of each crystal, concentrations generally reach their most extreme. There is a general progressive decrease in $\mathrm{HREE}+\mathrm{Y}$ central peak concentration with decreasing crystal radius (with one exception, discussed below), and rim compositions are comparable for a given element in all crystals. Zoning of two of the largest crystals, CT252 and CT262 (with radii close to $700 \mu \mathrm{m}$ ) is very similar: $\sim 1500 \mathrm{ppm} \mathrm{Y,} \sim 530 \mathrm{ppm} \mathrm{Lu}, \sim 2000 \mathrm{ppm}$ $\mathrm{Yb}, \sim 120 \mathrm{ppm}$ Tm, 450 ppm Er, 40 ppm Ho and 60 ppm Dy in their central peaks. The core of the smallest analysed crystal contains $\sim 400 \mathrm{ppm} \mathrm{Y,}<10 \mathrm{ppm} \mathrm{Lu}$, $\sim 50$ ppm Yb, <10 ppm Tm, 40 ppm Er, 15 ppm Ho and 50 ppm Dy. With decreasing atomic number, concentration maxima decrease, and elements with higher atomic numbers contain more significant relative concentration differences between the core compositions of large and small crystals (e.g., Lu compared to Ho, Fig. 3.11). Furthermore, HREE $+\mathrm{Y}$ are enriched in the cores of smaller garnets relative to their concentrations at the equivalent distance from the rims of larger garnets (Fig. 3.11).

In each garnet, patchy-to-sigmoidal cores are succeeded towards the rims by concentric HREE + Y oscillations approximately parallel to dodecahedral crystal surfaces (Figs. 3.3 to 3.10). Each garnet also contains several pronounced annuli that form sharp peaks at significantly higher concentrations than the background oscillations. 
Along a line profile within a given crystal, there is no spatial migration of the peaks according to atomic number, converse to that observed by Skora et al. (2006) and Moore et al. (2013). There is no coincidence between the position of annuli and notable zoning features in the major elements, $\mathrm{Zr}$ or P. Li zoning is comparable to that of the HREE $+Y$, with evidence for concentric Li annuli surrounding an enriched core region (Figs. 3.3 to 3.5). This likely results from the coupling of Li to HREE+Y in order to maintain electroneutrality during element substitution (Cahalan et al. 2014).

$\mathrm{Y}$ and Mn contents were evaluated along distinct annuli within all six crystals (Fig. 3.12 a), indicating that $\mathrm{Y}$ and $\mathrm{Mn}$ is approximately constant in concentration along the length of each annulus (subject to variations associated with raster direction versus profile direction and signal smearing). Similarly, the pyrope fraction at Y peaks along major element EPMA profiles in Fig. 3.1p shows approximate chemical symmetry about the elevated core (Fig. 3.12b). Figures $3.12 \mathrm{a}$ and b indicate that each annulus is compositionally distinct with respect to major and trace elements.

The geometries and relative locations of annuli in each crystal are variable, and crystals are therefore not easily comparable. The three annuli in CT252 are all located in close proximity to the rim, whereas in CT253 the annular transition is shifted towards the core (Fig. 3.12 ). CT262 contains three irregular annuli similar to CT252, which have an orientation and structure at odds with the satellite peaks parallel to the dodecahedral surface situated towards the rims (Fig. 3.4). The latter forms annuli approximately parallel to present crystal interfaces, whereas the former is rotated relative to the present crystal surface. FE-EPMA maps of the largest garnet (CT252) indicate that the three dominant Y annuli (and by association, annuli of the other HREE) are characterised by sharp increases moving from core-to-rim, with a smoother decrease on the rim-ward side of each annulus (Fig. 3.10b). 
CT253 is unique in that much of the relatively systematic, size-dependent trace element behaviour documented in other garnets is not present. Despite being of comparable radial size to the largest garnet, core compositions of HREE $+\mathrm{Y}$ are similar to the magnitude of the smallest crystal, CT246: core Lu values in CT253 do not exceed 20 ppm (Fig. 3.11 ), and Y reaches a maximum of $\sim 300$ ppm (Fig. 3.9 d). However the particularly distinct annuli in CT253 are significantly more elevated relative to the core composition. Perhaps unexpectedly, major element zoning of this garnet is consistent with the systematic size-composition relationship documented within other crystals.

\section{Middle rare-earth elements and light rare-earth elements}

There is inversion in the sense of zoning in the MREE compared with the HREE, with core-to-rim increases in the concentration of $\mathrm{Gd}, \mathrm{Sm}$ and $\mathrm{Eu}$, and a near homogeneous core-to-rim distribution of Tb. Unlike the significant size dependence of the HREE, MREE zoning is less sensitive to crystal size, although some fractionation from large to small crystals is still notable in Gd. There are core-to-rim increases from $\sim 0.3$ to $\sim 0.9-1.2 \mathrm{ppm}$ and from $\sim 0.5$ to $\sim 1.5 \mathrm{ppm}$ for Eu and Sm, respectively (Fig. $3.9 \mathrm{~b}-\mathrm{g}$ ). Gd is more notably zoned, with core compositions of 2-4 ppm and rims of 11-13 ppm in all garnets. Average $\mathrm{Tb}$ concentrations are approximately constant at $3-5 \mathrm{ppm}$ from core-to-rim in all garnets.

Despite little systematic rim-to-rim zoning, $\mathrm{Tb}$ is the only MREE that shows evidence for pronounced compositional change that is spatially coincident with the position of HREE $+\mathrm{Y}$ annuli. Line profiles reveal that some correlative peaks and troughs are also present in Gd and Eu. Some MREE peaks are non-correlative with HREE features. Despite lower than anticipated HREE concentrations in CT253, the MREE do not exhibit any depletion relative to other similar sized garnets. 
Maps of light rare-earth elements (LREE: Ce, Pr and Nd) do not record any systematic crystallisation-related zoning, or are zoned at a scale less than detectable by LA-ICP-MS. LREE record the presence of accessory inclusions in the garnets as a series of saturated patches.

\section{Transition elements and other metals}

Cr zoning is unique amongst the mapped elements: in all garnets Cr exhibits continuous spiral zoning that traverses from core-to-rim, with spirals centred on the compositional and geometric core of each crystal (Fig. 3.13). The zoning is defined by two approximately discrete regions: a high-Cr and a low-Cr domain. Low-Cr domains contain between 20 and 55 ppm Cr. Towards garnet rims, high-Cr zones become increasingly enriched, with values reaching 160 ppm Cr, compared to 100-120 ppm nearer the core (Figs. 3.3 to 3.8. CT176 has high- and low-Cr zones but does not document continuous spiral zoning, likely resulting from a poorly oriented section relative to the spiral geometry. Maps also show poorly defined oscillatory Cr zoning parallel to HREE+Y.

Spiral Cr zoning shows remarkable parallelism with the sigmoidal inclusion fabrics in garnet. As illustrated in Fig. 3.13, high-Cr zones strongly correlate with regions containing inclusions of ilmenite, allanite and apatite, whereas areas of low-Cr correspond almost exclusively to flattened quartz inclusions. This is the case in garnets of all sizes, regardless of degree of apparent rotation. The orientation of these Cr spirals is also correlative with the sigmoidal patterns in HREE $+\mathrm{Y}$ core regions and the curved patchy zoning in Zr. Ca zoning towards garnet cores (Fig. 3.1k) also exhibits evidence of a similar element distribution.

Sc, Ti and V each exhibit core-to-rim decreases in concentration, with approximately constant rim compositions between crystals for a given element. Cores of the 
four largest crystals contain 160-200 ppm Sc, whereas the smallest contains $\sim 100$ ppm Sc. Ti allows for tracking of ilmenite inclusions in garnet. Each element approximately mimics the sigmoidal zoning observed in HREE and $\mathrm{Cr}$, with a more patchy distribution.

Hf concentrations are ubiquitously below detection limits, so any potential zoning cannot be constrained. Unlike the complex zoning exhibited by other trace elements, zoning of $\mathrm{Co}, \mathrm{Zn}$ and $\mathrm{Ga}$ is relatively simple in crystals of all sizes, not dissimilar to major element zoning. For each element, rims are approximately within error between crystal sizes, and there is no correlation between Co, $\mathrm{Zn}$ and $\mathrm{Ga}$ and the HREE+Y annuli. Co and Zn are characterised by broad core-to-rim increases, from $\sim 10 \mathrm{ppm}$ to $25 \mathrm{ppm}$ Co and $\sim 30$ to $65 \mathrm{ppm} \mathrm{Zn}$ in the largest crystals. There is also

progressive elevation of core values from large to small crystals. Ga concentrations broadly decrease from core-to-rim, with a maximum of $4 \mathrm{ppm}$ variation in the largest crystal.

\subsubsection{Matrix phase geochemistry}

Table 3.2 displays the major and trace element contents of muscovite, biotite and chlorite in sample 24-99. Analyses are the average of multiple grains, with an associated $\pm 1 \sigma$ error. Muscovite, biotite and chlorite contain $<0.02 \mathrm{ppm}$ Y and HREE below detection limits. Instead, these phases are relatively enriched in transition metals. All contain approximately similar Sc contents (17, 11 and $20 \mathrm{ppm}$ in muscovite, chlorite and biotite, respectively), whereas $\mathrm{Cr}$ and $\mathrm{V}$ are significantly more abundant (138 and 162, 134 and 105, and 213 and 203 ppm Cr and V in muscovite, chlorite and biotite, respectively). Sc, $\mathrm{V}$ and $\mathrm{Cr}$ vary significantly about mean values in each analysed phase, with the implication that the associated trace element contents of these phases are heterogeneous throughout the section. 
Chlorite and biotite contain relatively high concentrations of $\mathrm{Zn}$ and $\mathrm{Co}(\sim 976$ and $\sim 656 \mathrm{ppm}$, and $\sim 147$ and $\sim 107 \mathrm{ppm}$, respectively). Chlorite, biotite and muscovite contain similar Ga concentrations (45-65 ppm). The scarce ( $\sim 6$ vol $\%)$ plagioclase was not analysed with LA-ICP-MS, but is expected to be relatively enriched in LREE, Sr and $\mathrm{Ba}$ (e.g., Bea et al., 1994). Cr in plagioclase is generally $<10 \mathrm{ppm}$. Quartz was not analysed.

\subsection{Discussion}

\subsubsection{Origins of trace element distribution}

The following discussion is focused on several phenomena documented in the trace element zoning patterns presented above. Namely, the influence of progressive garnet nucleation and growth on the trace element distribution in garnet, in relation to crystal size and extent of chemical equilibration of the rock matrix; the kinematic and textural controls on the sigmoidal-to-spiral $\mathrm{Cr}$ and $\mathrm{HREE}+\mathrm{Y}$ zoning in garnet cores; and the formation mechanism of numerous concentric HREE $+\mathrm{Y}$ annuli. We also address the potential cause of the anomalous HREE compositions in garnet CT253.

\section{Size-dependent changes in zoning patterns}

Similarities between the core compositions of similar sized garnets CT252 and CT262 confirm their equivalent nucleation and early growth histories in regions of highest HREE, in a presumably undepleted precursor matrix. Patchy zoning in the cores

of crystals suggests that during low- $T$ growth, HREE $+Y$ behaved in the same way as Ca (Menard \& Spear, 1996, Chernoff \& Carlson, 1997, Daniel \& Spear, 1998), with reduced mobility compared to $\mathrm{Mn}, \mathrm{Fe}$ and $\mathrm{Mg}$, and a lack of hand sample scale 
equilibration. The curved patterns in the core HREE+Y zoning (particularly evident in the largest crystals) appear to have been partially inherited from the trace element distribution present in the pre-existing fabric that was overprinted during growth.

The strong enrichment of HREE in restricted core regions of each garnet (Fig. $3.9 \mathrm{~b}-\mathrm{g}$ ) results from the fractionation of elements into the earliest formed portions of each garnet, with more pronounced enrichment for those elements with higher atomic numbers and partition coefficients. Coupled strong partitioning of HREE+Y into early-grown garnet and slow rates of diffusion in the matrix likely resulted in the development of a concentric diffusion halo with steep chemical potential gradients around each growing garnet in which the trace element concentration was depleted (e.g., see Carlson, 2006). Despite the development of depletion haloes, core regions are generally characterised by irregular chemical patterns, a relict of some precursor chemical texture. Thus, the early-incorporated chemistry of each garnet is as much controlled by the distribution of HREE, as the rate of their transport in the matrix.

As with the change in major element chemistry with crystal size, a general systematic change in the HREE $+\mathrm{Y}$ core compositions with size in the crystal population is further evidence of progressive nucleation and growth of large to small garnets. In addition to systematic changes in the absolute concentration of HREE+Y, the cores of small garnets are enriched relative to equivalent distances from the rims of larger crystals (Fig. 3.11). This is most notable for the heaviest HREE. Similar to Skora et al. (2006), this further confirms the presence of chemical concentration gradients in the matrix during garnet crystallisation, because each radial increment of late-nucleated smaller garnets grew contemporaneously with radial increments of early-nucleated larger garnets, as evinced by assessment of major element zoning George \& Gaidies, 2017).

This relative core enrichment may be attributed to several different phenomena. 
Firstly, small garnets may have nucleated in an already partially depleted matrix if diffusion haloes around previously grown garnets overlapped. Estimates of the characteristic diffusional length scale of HREE transport (defined as $l=\sqrt{D_{\text {eff }} t}$, e.g., Darken \& Gurry, 1953) with effective matrix diffusivities, $D_{\text {eff }}$, on the order of $10^{-18}$ to $10^{-23} \mathrm{~m}^{2} \mathrm{~s}^{-1}$, do not exceed $1.5 \mathrm{~mm}$ at the modelled crystallisation timescales, $t$, of $0.5-1.5$ Myr. While this is of a magnitude of the nearest-neighbour spacing of the final porphyroblast distribution, the relative scarcity of early-nucleated crystals would necessitate diffusion haloes at least an order of magnitude larger than this lengthscale, if they were to intersect and deplete the bulk matrix during the early stages of garnet crystallisation.

Alternatively, if slow diffusivities preclude diffusion haloes from overlapping and homogeneously depleting the average matrix chemistry during crystallisation, the progressive change in core concentration with garnet size requires there to have been a compositionally heterogeneous pre-existing matrix with respect to HREE+Y. The assumption of a chemically equilibrated low- $T$ precursor seems implausible, given the dependence of the effective diffusivity on $T$, the chemical variability in mica and chlorite even after peak metamorphic conditions (Table 1), and overprint zoning in the cores of garnet. Progressive nucleation and growth of garnet in a patchy, nonequilibrated precursor medium is also supported by the fact that regions of highest $\mathrm{HREE}+\mathrm{Y}$ in LA-ICP-MS maps do not converge in the geometric centre of annuli, and are often irregular in shape, despite approximate symmetry in line profiles.

In the case that the HREE+Y distribution in the matrix was heterogeneous and approximately immobile at $T$ 's of garnet crystallisation, the systematic change in core HREE+Y concentrations with garnet size may imply that the HREE+Y distribution had some direct or indirect control on the disposition of garnet nucleation sites. While it is generally well-established that trace elements are not an essential 
structural component in garnet, garnet may have preferentially nucleated in regions of highest HREE, with subsequent nucleation in sites of progressively decreasing HREE content. This population may therefore indicate that the trace element distribution and concentration may have had some impact on garnet nucleation, such that ease of nucleation scaled positively with the local concentration of HREE+Y.

While peaks and troughs are still present, the inverse core-to-rim MREE zoning does not show compositional change with crystal size to the extent documented by HREE $+Y$. This zoning can be attributed to the increasing incompatibility of MREE with decreasing atomic number (e.g., Bea et al., 1997; Kessel et al., 2005). Weak Tb zoning indicates garnet overprinted the bulk $\mathrm{Tb}$ distribution in the matrix rather than enhancing chemical potential gradients by strong fractionation. As a consequence, significant depletion haloes are unlikely to have developed for MREE.

As with major elements, the core contents of Co, Zn and Ga within smaller crystals correspond to concentrations of simultaneously grown rims in larger crystals. This, coupled with symmetrical smooth zoning of $\mathrm{Co}$, Zn and $\mathrm{Ga}$, and progressive core variation with crystal size (similar to that observed in major element zoning) suggests potential equilibrium partitioning of these elements into garnet rims during growth. It is possible that the greater abundance and more widespread distribution of Co, $\mathrm{Zn}$ and Ga in matrix phases (Table 1) means that sample-wide equilibrium is more readily achieved for these elements in the matrix. Furthermore, the cationic radii of Ga, Zn and $\mathrm{Co}$ in both six- and eight-fold coordination is, on average, $\sim 0.3-0.4 \AA$ smaller than the HREE (and similar to major elements), possibly facilitating an increased $D_{\text {eff }}$ through the grain boundary network and more effective chemical equilibration.

Broad core-to-rim changes in the concentrations of Co, Zn and Ga in garnet may also arise as a result of continuous adjustments to the garnet forming reaction over the duration of crystallisation. The broad zoning patterns observed in sample 24-99 
are comparable to those in Raimondo et al. (2017), who suggested that progressive relative depletion and enrichment of the HREE and lighter elements, respectively, in remaining matrix minerals over the duration of garnet crystallisation can account for zoning patterns. While a gradual change in the compositions of the reacting phases is not able to account for the sharp peaks observed in HREE+Y, progressive relative enrichment of lighter trace elements and transition metals in remaining reactant phases, and their subsequent participation in the garnet-forming reaction may contribute to core-to-rim bowl shaped increases in some elements. Over the duration of garnet crystallisation, phase equilibria modelling indicates that modal abundance of plagioclase and chlorite decreased from 5 to 3 vol.\% and 14 to 10 vol.\%, respectively (George \& Gaidies, 2017), increasing the supply of Co and Ga (from chlorite in particular) towards the end of the growth sequence.

\section{Formation of spiral zoning}

Precursor matrix foliations comprising alternating quartz-plagioclase microlithons and phyllosilicate-rich cleavage domains likely yielded alternating regions of relatively low- and high-Cr, respectively. Micas typically contain tens to hundreds of ppm $\mathrm{Cr}$ (Bea, 1996; Yang et al., 1999; Yang \& Rivers, 2001; Pyle et al., 2001; Corrie \& Kohn, 2008; Janots et al., 2008), and LA-ICP-MS analysis indicates that muscovite, biotite and chlorite in sample 24-99 contain 100-240 ppm Cr, comparable to the concentration of Cr in high-Cr garnet domains. Petrographic assessment indicates that cleavage domains are also the predominant host of accessory phases, which may provide an additional source of Cr. Given the strong correlation between the inclusion populations and $\mathrm{Cr}$ content in garnet, it is inferred that the $\mathrm{Cr}$ distribution was controlled principally by the geometry of these precursor phyllosilicate layers. V and Sc distributions in garnet were likely also controlled by this strongly heterogeneous precursor 
rock texture.

Overprint Cr zoning has previously been documented in metapelitic garnets that have experienced conditions less than $c .600{ }^{\circ} \mathrm{C}$ (Yang \& Rivers, 2001; Martin, 2009, Raimondo et al., 2017). Cr in migmatitic garnets records concentric zoning, likely related to enhanced mobility of $\mathrm{Cr}$ at higher T (Yang \& Rivers, 2001), or persistent disequilibrium between coexisting garnet and melt (Jung \& Hellebrand, 2006). Studies of amphibolite grade spiral $\mathrm{Cr}$ zoning in garnet has resulted in the widespread application of a model of garnet growth from two radial growth faces at a notch that remains approximately static in quartz pressure shadows as garnet grows and is rotated (Yang \& Rivers, 2001), in order to account for relatively high- to low-Cr zones. However, coeval development of $\mathrm{Cr}$ spirals and concentric HREE+Y annuli preclude a non-concentric growth model in the garnet porphyroblasts from Sikkim. Instead, the partially decoupled zoning patterns indicate garnets underwent approximately concentric growth, with continued rotation over the duration of crystallisation.

Spirals and sigmoids defined by inclusion trails that formed during non-coaxial deformation have historically been interpreted to result from one of two end-member models: (1) rotation of porphyroblasts during growth due to significant coupling between the garnet and matrix interface (Rosenfeld, 1970, Schoneveld, 1977, Passchier et al., 1992), or (2) orthogonal reorientation of the external foliation around nonrotating porphyroblasts, in which deformation of the matrix is partitioned around the porphyroblast (e.g., Bell, 1985; Bell \& Johnson, 1989; Hickey \& Bell, 1999). Although garnets have been isolated from their matrix in this study, the continuous Cr zoning strongly supports the former scenario, as there is little chemical evidence in garnet for the degree of misorientation of the matrix or of truncation of the internal foliation required by the non-rotational model. This kinematic record of continuous rotation during growth would not be evident from patterns of major elements or REE alone, 
which may otherwise suggest a cessation of rotation at conditions corresponding to the $P-T$ of formation of the first annuli in each crystal.

Continuous spiral zoning from core-to-rim and lack of local chemical equilibration along the interface of growing garnet necessitates approximate immobility of $\mathrm{Cr}$ in the intergranular medium surrounding garnet during syn-kinematic growth. As discussed by Yang \& Rivers (2001), Cr tends to become enriched in remnant phases due to its resistance to substitution reactions and relative insolubility in grain boundary fluids (Burns, 1993), comparable to the immobile behaviour of $\mathrm{Al}$ in metapelites. The observation that $\mathrm{Cr}$ is unable to achieve even local equilibrium and preserves precursor $\mathrm{Cr}$ variations is therefore unsurprising. However, elevations of $\mathrm{Cr}$ towards the rims of crystals suggest either a gradual increase in the mobility of Cr parallel to pre-existing cleavage domains, or a progressive change in the partitioning of Cr into garnet with increasing $T$, or both. Slightly enhanced mobility of $\mathrm{Cr}$ at higher $T$ is also supported by the presence of oscillatory Cr zoning parallel to $\{011\}$ planes near garnet rims.

\section{Overprint to annular transition}

In each mapped garnet the first annulus generally marks the transition from overprint zoning to concentric zoning parallel to the primary garnet-matrix interface. The concentric and linear nature of annuli is consistent with their formation during primary crystallisation, as opposed to forming in response to a resorption and regrowth episode (e.g., Pyle \& Spear, 1999; Kelly et al., 2011).

In the case that there was major element equilibration between garnet rims and the rock matrix, the concentrations of equilibrated components in garnet can be used as a relative timeline for garnet growth. The spessartine content is often used for such analysis (e.g., Finlay \& Kerr, 1979, Carlson, 1989), and previous studies have 
used major elements to interpret annuli as rock-wide event markers, such that they formed at the same time in all crystals. However in an alternate case, Chernoff \& Carlson (1997) concluded that Ca-spikes demonstrably did not form at the same time in all crystals, and are instead related to an event associated with the crystallisation history of each individual garnet crystal.

Equilibrium forward modelling of the garnet compositions and sizes in 24-99 yields a good fit for all major elements (George \& Gaidies, 2017). However, for large crystals, simulated concentrations of $\mathrm{Mn}$ in core regions are over-predicted (Fig. 3.1p), whereas the same compositions in small crystals are effectively reproduced. This may be attributed to uncertainties of equilibrium phase relations at low- $T$ or spherical shape effects of the model, but may also point to a lack of equilibration of Mn between crystals during early growth, such that radial fractions grown at the same time incorporated different Mn concentrations. Thus, the use of Mn composition as a comparative timeline for annulus formation may be unreliable for garnets in 24-99. As Mg zoning is effectively reproduced by the modelling (Fig. 3.1 b), the pyrope content in garnet may serve as an additional appropriate timeline. Given that rims of crystals are interpreted to have formed simultaneously, the distances from the rim to the most core-ward annulus can be used as a relative timeline for the transition from patchy zoning to concentric oscillatory zoning in different crystals.

As can be seen in Fig. 3.12 and b, characteristic annuli formed across a range of Mn, Mg and Y compositions (c. 1500-26000 ppm Mn, c. 0.046-0.075 mole fraction pyrope, and c. 110-520 ppm Y) and at a range of distances from crystal rims (Fig. 3.12 c). Compositional constraints in Fig. 3.12a and b indicate that some annuli may have formed approximately simultaneously: a number of discrete annuli in different crystals formed at c. $0.054,0.060$ and 0.065 mole fraction pyrope, and large crystals with similar element concentrations and modelled crystallisation histories (CT252 and 
CT262) contain comparable Mn at each of their annuli. This major element compositional correspondence between different annuli may point to their formation at similar times. However, there is by no means a systematic chemical trend throughout the population, making it unlikely that annuli are genetically related to some simultaneous event. Annuli that may have formed at comparable times display disparate Y concentrations, indicating a lack of chemical equilibrium for Y over the distances separating such crystals. Conversely, annuli with the same approximate Y contents formed at variable pyrope contents, further supporting a space- and time-dependent HREE $+Y$ distribution in the vicinity of each garnet.

There is no correlation between the major element concentrations in the cores of garnet and the Mn concentrations at the first annulus (as observed in Chernoff \& Carlson, 1997). Thus it is unlikely that there is a systematic relationship between the time of nucleation of a given crystal and the time at which its first annulus was generated. In other words, the annuli are not tied to the relative extent of the porphyroblastic reaction progress of individual crystals.

The lack of definitive correspondence between equilibrated major element chemistry at annuli in different garnets likely precludes their formation at the same time in some rock-wide event, or their systematic formation at a particular stage in each garnet's crystallisation history. While annuli are not rock-wide markers, the relative constancy of annuli composition along their lengths (Fig. 3.12a) and on either side of rim-to-rim compositional profiles (Fig. 3.12b) suggests that each given annulus is approximately equilibrated along its 2D length, and thus across the 3D surface of the growing garnet. The lack of $\mathrm{HREE}+\mathrm{Y}$ equilibration in the vicinity of growing garnet, coupled with this continuous nature therefore necessitates some increase in the rate of grain boundary diffusion along the garnet surface at $P-T$ conditions of formation of the first annuli, relative to those conditions at which patchy zoning 
in garnet cores formed. Given the assumptions discussed, Fig. $3.12 \mathrm{k}$ indicates that CT252, CT253, CT262 and CT176 experienced this transition simultaneously, at a distance approximately $190-270 \mu \mathrm{m}$ from crystal rims.

\section{Annuli formation mechanism}

Discontinuous changes in $\mathrm{Y}$ and other REE contents in garnet have previously been ascribed to numerous formation mechanisms, including changing stability of major and accessory phases resulting in an increased availability of trace elements (Hickmott et al., 1987, Konrad-Schmolke et al., 2008, Moore et al., 2013); diffusion-limited uptake (Skora et al. 2006); infiltration of trace element enriched fluids (Hickmott \& Spear, 1992, Stowell et al., 1996); and disequilibrium partitioning in the vicinity of the interface and growth entrapment due to slow element diffusivities (e.g., Hickmott \& Shimizu, 1990, Lanzirotti, 1995; Watson \& Liang, 1995, Watson, 1996, Watson \& Müller, 2009). Given the caveats discussed pertaining to the HREE+Y distribution and relative timing of annuli formation, the applicability of these scenarios to the sample studied here are discussed below.

Phase assemblage changes Phase equilibria modelling and garnet-growth simulations coupled with detailed petrography indicate that the garnet population in 24-99 crystallised in a single graphitic assemblage containing muscovite, chlorite, quartz, plagioclase and ilmenite (George \& Gaidies, 2017). Consequently, discrete assemblage changes are not thought to be able to account for the observed changes in HREE $+\mathrm{Y}$, as in the model of Konrad-Schmolke et al. (2008).

Pyle \& Spear (1999) attributed shallow core-ward annulus gradients to progressive supply of HREE from discontinuous accessory phase reactions. However, steep compositional gradients on the core-ward side of annuli and shallow on the rim-ward 
side in 24-99 garnets (Fig. 3.10p) are inconsistent with a gradually increased supply of HREE and sudden decrease when the phase has reacted and the element supply is exhausted.

Regardless of the geometry of annuli, sample 24-99 is limited in its variance of trace element enriched accessory phases, with only ilmenite, zircon, apatite and allanite present in both the matrix and garnet. Neither monazite nor xenotime, both commonly saturated in HREE, are present and are hence considered unlikely candidates for the source of elevated HREE observed at annuli. While apatite can also act as a moderate sink for $\mathrm{Y}+\mathrm{REE}$ (e.g., Heinrich et al., 1997), there is no notable variation in $\mathrm{P}$ in the garnets. No peaks are observed in LREE, making allanite an unlikely source of the HREE variation. Zircon breakdown would undoubtedly provide a significant source of $\mathrm{HREE}+\mathrm{Y}$ to the advancing interface, but volumetrically significant release of $\mathrm{Zr}+\mathrm{HREE}$ resulting from the dissolution of zircon in metapelites is unlikely to occur until the onset of partial melting (Kohn et al., 2015).

Diffusion-limited uptake The spatial correspondence of annuli of different HREE $+Y$ in a given crystal, the geometry of the sharp peaks observed in the studied garnets, and the lack of annuli for the majority of MREE precludes the application of the diffusion-limited uptake model of Skora et al. (2006). While core HREE zoning trends are similar to those modelled by Skora et al. (2006), the growth of garnet in 24-99 may have occurred over too short a timescale to enable sufficient diffusion and re-enrichment of HREE in the vicinity of growing garnet to account for secondary peaks. In their model, a central peak only develops when the pre-exponential diffusion factor, $\mathrm{D}_{0}$, is on the order of $10^{12}-10^{13} \mathrm{~cm}^{2} \mathrm{y}^{-1}$, and rates of intergranular diffusion required for annular peaks to form are unrealistically rapid for the case of trace elements in 24-99. 
Fluid-mediated changes The increased relative solubility of trace elements in metamorphic and metasomatic fluids yields the potential for significant fluxes of REE through a reacting rock during prograde metamorphism. As discussed by Haskin et al. (1966) and Hickmott et al. (1997), the solubility of HREE can be radically increased particularly in a $\mathrm{F}$ - or $\mathrm{CO}_{2}$-rich fluid. However, the repeated nature of annuli would require multiple fluid infiltration events, yielding some cyclic heterogeneous fluid flux across the surfaces of growing garnets. Repeated infiltration of fluids cannot be definitively ruled out, but there is no evidence for a compositionally distinct fluid or significant migration of fluid through the rock. Furthermore, mobility of major elements would similarly be expected to increase in a fluid-rich system. The success of major element simulations suggests that closed system behaviour closely approximates the effective bulk chemistry available during garnet growth.

Growth entrapment and fluctuating growth rates Given the evidence that the HREE+Y distribution in the matrix was not broadly equilibrated over the distances separating the analysed crystals during garnet growth, it can be expected that there were persistent regions of irregular HREE+Y content across the matrix. Disequilibrium partitioning has previously been shown to be possible at the surface of a growing crystal due to near surface enrichment or depletion of components with slow diffusivities (e.g., Hall, 1953). Upon significant increases in growth rates, trace elements in the surface region can become bound in the surface layer, resulting in lattice concentrations that deviate from those expected with equilibrium partitioning. Preservation of such short-range compositional heterogeneity is dependent on the rate of interfacial advance relative to the rate of diffusion in the matrix and in the crystal: the ratio of diffusivity to growth rate is described by the growth Péclet number, and can be calculated as the product of the growth rate and lengthscale of 
diffusion, divided by the component diffusivity (e.g., Tiller, 1991).

In a system defined by a high Péclet number (i.e. slow diffusion relative to growth rate), the rate of interfacial advance may exceed the rate of radial propagation of diffusion haloes, such that matrix heterogeneities are directly incorporated by growing garnet. Given the exceedingly rapid rates of radial growth predicted by the simulations, on the order of $10^{-19} \mathrm{~m} \mathrm{~s}^{-1}$ (George \& Gaidies, 2017), this scenario is considered likely. Rapid garnet growth rates in 24-99 prevents significant equilibration of HREE+Y before heterogeneous matrix compositions are overprinted. However, unlike the apparently negligible diffusion in the intergranular medium, formation of annuli upon interface infringement of enriched or depleted zones in the matrix requires the diffusivity of $\mathrm{HREE}+\mathrm{Y}$ parallel to the garnet-matrix interface have been extremely rapid, so as to allow for approximate equilibration along this $3 \mathrm{D}$ surface. Elevated T's in the latter stages of growth (coupled with potential effects from changing element solubility in the grain boundary medium) may have facilitated this.

Minor fluctuations in the rate of radial garnet growth may also have had a dramatic effect on the compositional record of growth entrapment. As discussed by Watson \& Müller (2009), for components with slow diffusivities, rapid mineral growth is expected to result in the accumulation of incompatible elements at the crystal-matrix interface. Conversely, compatible elements are expected to be at a minima at the interface, and increasingly enriched into the matrix. Sudden discrete increases of an already rapid interfacial advance rate may result in the growth entrapment of the matrix concentration gradient in the new surface layer of garnet. This would yield a sharp core-ward side and a shallower gradient towards the rim, as is observed in 2499 (Fig. 3.10b). However as compatible HREE are significantly enriched at annuli in garnets of 24-99, the increased growth rate model is not compatible with observations.

Conversely, as suggested by Lanzirotti (1995) and as shown to be the case for 
$\mathrm{MnO}$ in garnet by Edmunds \& Atherton (1971) and Burton et al. (1951), a decrease in the crystal growth rate will yield an increase in the concentration of compatible components at the surface of the growing crystal. For garnets in a heterogeneous matrix, discontinuous slowing of garnet growth could permit build up of compatible elements at the interface, and renewed rapid growth rates can subsequently overprint the elevated concentrations.

The oscillating nature of zoning towards the rims of garnets may therefore be associated with changes in growth rate. Phases of rapid growth will cause local depletion in trace element concentration, while a slowing of growth will allow build up of trace elements at the interface. Such changing growth rates may be driven by stepwise $P-T$ changes, resulting from cyclic changes in slip rates along local faults Kohn, 2004). Shear motion in the vicinity of the MCT zone is commonly invoked to explain the rapid heating and metamorphic mineral growth in the Sikkim Barrovian sequence (e.g., Le Fort, 1975; Burg \& Chen, 1984; Mottram et al., 2014b), and may have acted as an external driver of fluctuating garnet growth rates.

\subsubsection{Influence of rock texture on chemical zoning}

While the systematic zoning of transition elements (excluding $\mathrm{Cr}$ ) and the less abundant MREE may provide evidence for their approximate intergranular equilibration during mid-amphibolite facies metamorphism, persistent rock-wide disequilibrium with respect to $\mathrm{HREE}+\mathrm{Y}$ is indicated by the irregularity of the zoning patterns. In addition to evidence for depletion haloes surrounding growing garnets, the variable form and timing of annuli development and the anomalous core composition of CT253 suggests that effective HREE bulk compositions may be significantly partitioned into a series of chemically distinct zones within 24-99. Cr also provides evidence of a strongly heterogeneous precursor rock texture. 
The similarities between general zoning patterns and absolute concentrations of trace elements in CT252 and CT262 suggest a shared chemical history for some crystals. Micro-CT scans indicate that the cores of these crystals are separated by 4.6 $\mathrm{mm}$ within a shared foliation plane. It is likely that the aforementioned garnets experienced similar crystallisation histories (i.e. with formation of annuli at approximately similar times) due to their distribution in an approximately similar chemical system, which must exist at least over the distance separating their cores.

Conversely, CT253 (which has a similar crystallisation history but exhibits unusually low HREE + Y concentrations and a contrasting annular pattern) is situated closer to CT252, but perpendicular to the foliation plane containing CT252 and CT262. Anisotropic intergranular diffusion in the rock matrix may therefore inhibit diffusive equilibration of HREE perpendicular to a spaced foliation, such that different local rock domains separated by as little as $4 \mathrm{~mm}$ contain significantly different HREE+Y concentrations, even prior to the onset of garnet growth. Micro-scale variations in effective bulk composition have been proposed previously (Stüwe, 1997, Carlson, 2002, Habler et al., 2009), resulting from differential diffusivities or strain induced heterogeneities. It may be the case that in 24-99, variations in the HREE+Y contents persisted in local domains defined largely by a strongly heterogeneous rock texture. Thus, the chemical system in which CT253 grew may have been dissimilar to that of CT252 and CT262, with respect to some elements only.

\subsubsection{Kinematics of porphyroblast rotation}

Given the apparent continuity of porphyroblast rotation during growth, Cr spirals can be used to estimate strain rates that acted over the duration of garnet crystallisation. The lack of in situ matrix surrounding fragmented garnet precludes the calculation of strain rates in the non-rotational scenario (e.g., Berg et al., 2013), but internal 
fabrics can be used to derive estimates of strain rates for the porphyroblast rotation end-member case. This method follows that outlined by Biermeier \& Stüwe (2003) and Berg et al. (2013), and relies on the relationship between strain rate and rotation,

$$
\dot{\gamma}=\left(\frac{h}{\Delta T}\right) * 2 \omega,
$$

where $\dot{\gamma}$ is the strain rate, $h$ is heating rate, $\Delta T$ is the change in temperature during a given interval of garnet growth, and $\omega$ is the magnitude of rotation over the interval. The crystallisation modelling of George \& Gaidies (2017) provides a good basis for the variables required for this analysis. The correspondence between inclusion trails and inferred rotation-derived $\mathrm{Cr}$ spirals permits digital reslicing of single crystal $\mu$-CT garnet datasets in order to evaluate the maximum inclusion curvature in the garnet crystals. In each case the plane showing the maximum curvature is approximately orthogonal to the elongation of the crystal. The amount of rotation, $\omega$, experienced by each crystal was constrained by measuring the angle between the tangential surfaces to the average surface of rotation (Fig. $3.13 \mathrm{~b}$ and Table 3.1). Equilibrium modelling of major element zoning has shown to be consistent with progressive nucleation of large to small crystals over the crystallisation interval. This allows approximate $P-T-t$ durations of growth of different crystal CSD size classes to be constrained. Volume-radius relationships for spherical crystals in the best-fit model require that early-formed growth zones grew exponentially more rapidly than late garnet shells, with a cumulative interval of $\sim 46{ }^{\circ} \mathrm{C}$ for core-to-rim growth of the largest garnet and $\sim 33{ }^{\circ} \mathrm{C}$ for the smallest analysed (see Fig. 11 of George \& Gaidies (2017)).

Diffusion modelling of major element zoning has yielded minimum heating rates of $100{ }^{\circ} \mathrm{C} \mathrm{Myr}^{-1}$ (George \& Gaidies, 2017). With the knowledge that diffusion modelling commonly yields minimum growth duration estimates (c.f. Viete \& Lister, 2016), this 
may represent the most rapid end-member of plausible rate estimates. A maximum duration of growth of 3.8 Myr derived from core and rim Lu-Hf geochrononological constraints on a kyanite zone metapelite (Anczkiewicz et al., 2014) was used to place an upper end-member on the potential duration of garnet growth. For these calculations, a modified Equation 1 was applied, as no $\Delta T$ was directly available.

For a constant heating rate of $100{ }^{\circ} \mathrm{C} \mathrm{Myr}^{-1}$, different crystal-integrated strain rate estimates are of a similar order of magnitude for large to small garnets: between $9.6 \times 10^{-12} \mathrm{~s}^{-1}$ and $1.8 \times 10^{-11} \mathrm{~s}^{-1}$. Average strain rates for the largest crystal is between $9.6 \times 10^{-12} \mathrm{~s}^{-1}$ and $1.2 \times 10^{-13} \mathrm{~s}^{-1}$, depending on the end-member rate chosen; the strain rate for the smallest crystal is between $1.8 \times 10^{-11} \mathrm{~s}^{-1}$ and $1.7 \times 10^{-13} \mathrm{~s}^{-1}$.

The apparent order of magnitude similarity between crystal-integrated strain rates is complicated given that small garnets nucleated after large garnets, but continued to grow with the same radial growth rate as larger ones for a given $P-T$ segment. The initial stages of garnet growth, recorded only in core regions of large, early-nucleated crystals, were much more rapid than later shared growth intervals (George \& Gaidies, 2017). In a simple case in which homogeneous strain operated over the whole rock volume, one would therefore expect late-nucleated garnet to record less total $\omega$ than early-nucleated ones, given the reduced $\Delta \mathrm{T}$ over their crystallisation. The radial growth rate of earliest nucleated crystals over the initial $13{ }^{\circ} \mathrm{C}$ of their crystallisation was exceedingly rapid, with reduced time available for rotation. However during the c. $33{ }^{\circ} \mathrm{C}$ of shared history between the largest and smallest mapped crystals, objects might be expected to rotate simultaneously. This is not the case for garnets from 24-99; smaller crystals consistently contain larger $\omega$ than larger ones (Table 3.1). The lack of curvature correlation between objects partially growing at the same time therefore necessitates variable rotation operating over short length scales within the rock volume. 
A textural and/or chemically irregular matrix is likely to result in a heterogeneous distribution of strain in the rock volume, yielding different apparent strain rates at the grain-scale. Large scale variations in rotation may be explained by slight variations in shape or orientation of crystals (Passchier et al., 1992; Johnson et al., 2006; Johnson, 2009, or the interactions between rigid objects in a deforming matrix (Ildefonse et al. 1992). Previous strain rate assessment (Barr \& Houseman, 1996, Biermeier et al., 2001; Biermeier \& Stüwe, 2003) has indicated the significant effect of porphyroblast texture on the rotation rate of cylindrical objects, and consequently, the calculated strain rate. The generalised relationship $\dot{\omega} \propto 0.5 \dot{\gamma}$ (from Equation 1, where $\dot{\omega}$ is rotation rate) used is highly dependent on both the number and arrangement of rigid objects, and the boundary conditions of the system, and can vary between $\dot{\omega}$ $\propto 0.25 \dot{\gamma}$ and $\dot{\omega} \propto 0.75 \dot{\gamma}$, depending on the porphyroblastic distribution (Biermeier \& Stüwe, 2003). Elevated strain rates in some cases can correlate with a change in rock chemistry, and may be the result of a metamorphic fluid that both modifies the chemistry and induces strain softening (e.g., Zulauf \& Helferich, 1997). Rocks in shear zones do not behave uniformly and most likely, the rheology of the matrix controls heterogeneous strain at the grain scale. Deformation partitioning into discrete zones of the matrix, each with variable components of simple shear may have yielded different deformation domains for different garnets (e.g., Bell, 1985, Paterson \& Vernon, 2001 Griera et al., 2011).

The time-averaged strain rate estimates derived in this study for the garnet zone of Sikkim are an order of magnitude higher than previous indirect strain rate estimates from similar regionally metamorphosed terranes. Berg et al. (2013) used similar methods to derive strain rates on the order of $10^{-14} \mathrm{~s}^{-1}$ for quartzose pelites from the Passo del Sole, and most regional strain rates are on the order of $10^{-15}$ to $10^{-13} \mathrm{~s}^{-1}$ (e.g., Holm \& Selverstone, 1990; Müller et al., 2000; Biermeier \& Stüwe, 2003). While 
full treatment of the strain history and associated textural development is beyond the scope of this discussion, the $10^{-13}$ to $10^{-12} \mathrm{~s}^{-1}$ strain rate estimates in Sikkim are broadly in agreement with those derived using piezometery by Ghosh et al. (2016), on rocks associated with motion along the Rangit Thrust. In Sikkim the location of both the MCT zone and the Rangit Thrust is highly contested (e.g., Catlos et al., 2004; Mottram et al., 2014a), but a zone of pervasive deformation may persist through the Daling group Barrovian sequence. Bhattacharyya (2010) and Bhattacharyya \& Mitra (2009) calculated strain rates on the order of $10^{-12} \mathrm{~s}^{-1}$ using samples from their mapped MCT2, to the north of 24-99. While there is no evidence for localised shear at the 24-99 locale, strain rates on the order of those predicted along high-strain zones indicate that pervasive deformation was significant.

\subsubsection{Implications for garnet geochronology}

As garnet exerts strong fractionation of $\mathrm{Lu}$ and $\mathrm{Sm}$ from $\mathrm{Hf}$ and $\mathrm{Nd}$, respectively, ${ }^{176} \mathrm{Lu}^{-176} \mathrm{Hf}$ and ${ }^{147} \mathrm{Sm}-{ }^{143} \mathrm{Nd}$ garnet geochronology is commonly used in order to constrain timescales of metamorphic mineral growth in a wide range of high-temperature rocks (e.g., Duchêne et al., 1997; Scherer et al., 2000; Smit et al., 2013, Anczkiewicz et al., 2014). The development of high-resolution micro-sampling techniques (e.g., Pollington \& Baxter, 2011; Dragovic et al., 2015) permits an ever-improving spatial resolution of garnet ages, such that growth zones can potentially be resolved in great detail. However, when bulk garnet ages are required, an understanding of the spatial distribution of trace elements within garnet splits is necessary in order to correctly interpret the obtained dates (e.g., Lapen et al., 2003; Skora et al., 2009; Kohn, 2009; Kelly et al., 2011). Due to strong preferential fractionation of the parent isotope into the core of garnet (e.g., Otamendi et al., 2002; Kohn, 2009), ${ }^{176}{ }^{\mathrm{Lu}}{ }^{176} \mathrm{Hf}$ garnet geochronology is usually thought to date the early stages of garnet nucleation and 
growth. ${ }^{147} \mathrm{Sm}-{ }^{143} \mathrm{Nd}$ dates are often younger than $\mathrm{Lu}-\mathrm{Hf}$ dates, either related to the lower closure temperature for the former or fractionation of Sm into the rims of garnet.

The results of this study, as those of Skora et al. (2006) and Raimondo et al. (2017), highlight the importance of integrated trace element assessment to ensure the correct and meaningful interpretation of $\mathrm{Lu}-\mathrm{Hf}$ and $\mathrm{Sm}-\mathrm{Nd}$ ages. In the case of garnets from 24-99, core-to-rim line profiles corrected for the effect of an approximately spherical geometry (Fig. 3.14) indicate that while concentrations are highest in the cores, much of the total Lu (and Y) contained within each crystal - the concentration per volume unit of garnet - is contained near the rims because of the increased volume effect at successive radial increments. In CT252 an annulus at $\sim 400 \mu \mathrm{m}$ that is assumed to be equilibrated along the garnet surface (Fig. 3.14 a) produces a marked enrichment of $\mathrm{Lu}$ (and Y) at this distance from the core (Fig. 3.14 p), because it occupies a comparatively large volume fraction of the total garnet. The Lu in smaller crystals (CT241 and CT176) is also weighted towards the rims. Given the unzoned nature of Hf, volume bias in bulk ${ }^{176} \mathrm{Lu}^{176} \mathrm{Hf}$ ages may therefore record ages discordant to the true nucleation and early growth age: shifting ages to results that date annuli formation and the closing stages of garnet growth.

$\mathrm{Nd}$ is not systematically zoned in LA-ICP-MS maps, and there are no notable discontinuities or annuli in Sm. The bowl-shaped rim-to-rim zoning of Sm combined with the volume effect will increasingly skew $\mathrm{Sm}-\mathrm{Nd}$ ages towards rims of garnet. Coupled ${ }^{176} \mathrm{Lu}^{-176} \mathrm{Hf}$ and ${ }^{147} \mathrm{Sm}-{ }^{143} \mathrm{Nd}$ may significantly underestimate the duration of prograde metamorphism and decrease isochron precision.

While HREE annuli in 24-99 are not inferred to result from resorption (e.g., as in the case modelled by Kelly et al., 2011), the importance of selection of the appropriate garnet split is similarly emphasised by knowledge of the sample CSD and inferred 
history of crystallisation. A negatively skewed CSD resulting from the integrated history of nucleation and growth of garnet in 24-99 (Fig. 2a) means that much of the garnet volume is contained within fine crystal fractions, with generally lower absolute trace element concentrations in a given crystal. These small crystals are still volumetrically enriched in $\mathrm{Lu}$ in their rims relative to their cores (Fig. 3.14p). The models suggest that size-dependent interface-controlled growth best fits the observed major element zonation. This means that different garnets record different segments of the $P-T$ path, but rims of all crystals already nucleated grew simultaneously. Thus, large garnets record the longest history, whereas smaller crystals only document the final stages of crystallisation. Unbiased bulk separates of such a population might therefore be expected to yield little population-wide spread in ${ }^{176} \mathrm{Lu}^{-176} \mathrm{Hf}$ and ${ }^{147} \mathrm{Sm}-$ ${ }^{143} \mathrm{Nd}$ ages.

\subsection{Conclusions}

LA-ICP-MS maps of numerous garnets representative of a single rapidly crystallized population shed further light on the significant variability of the trace element distribution both within garnet, and in the intergranular medium from which garnet crystallised. The work suggests that garnets from the Sikkim Barrovian sequence contain an integrated record of the interplay between variable element equilibration, changing garnet growth rates and rock deformation, which would go otherwise unobserved with major element analysis alone.

During the early stages of garnet growth, HREE+Y exhibit a lack of rock-wide equilibration and a strongly space- and time-dependent element distribution. This immobility, coupled with relatively slow diffusivities in garnet yields compositional mimicry of the pre-existing matrix in the cores of garnets. Conversely, some tran- 
sition elements with greater matrix abundance exhibit approximate equilibrium behaviour, in part due to the shorter relative length scales required for homogenisation. Progressive change in core chemistry from large to small crystals attests to a progressive nucleation history, and may also point to preferential nucleation of garnet in HREE $+Y$ enriched regions. The transition from overprint to oscillatory zoning necessitates an increase in the length-scale of equilibration of HREE+Y along the garnet-matrix interface during the crystallisation interval. The timing and formation mechanism of these annuli is not systematic through the rock volume, but is likely a function of the interplay between inhomogeneous growth rates, the incorporation of pre-existing heterogeneities due to slow matrix diffusivities and rapid growth rates, and the influence of domainal HREE+Y bulk chemistries.

The crystallisation dynamics of syn-kinematic garnet are revealed by the compositionally defined internal foliation, which is most evident in the core-to-rim spiral zoning of Cr. Coeval development of spiral $\mathrm{Cr}$ and concentric REE growth zoning necessitates the approximately concentric growth of garnet porphyroblasts during continued garnet rotation, and a strongly heterogeneous but layered $\mathrm{Cr}$ distribution in the precursor matrix. Spiral zoning has proven to be well-suited to strain rate calculations: using thermobarometric and temporal constraints derived by George \& Gaidies (2017), we derive an average strain rate from $10^{-11}$ to $10^{-12} \mathrm{~s}^{-1}$, with significant sample-wide variation attributable to deformation partitioning in a texturally and microstructurally heterogeneous rock volume.

Persistent HREE $+Y$ disequilibrium in a sample that may be otherwise considered an archetypal prograde garnet population highlights the need for caution when using non-equilibrated trace elements for geochronological or geothermobarometric studies. Until the spatial distribution of trace elements in garnet populations is better understood, thorough investigation of each sample is required in order to prevent 
misinterpretation of single garnet chemistries. This study highlights the wealth of information that extensive datasets can provide in order to continue to improve our understanding of trace element distributions and garnet porphyroblast crystallisation. 


\section{Tables and Figures}


Table 3.1: Strain rate calculations for garnet in sample 24-99.

\begin{tabular}{lcccc}
\hline \multirow{2}{*}{ Crystal } & \multirow{2}{*}{$\left.\mathrm{T}^{\circ} \mathrm{C}\right)$} & $\omega\left(^{\circ}\right)$ & \multicolumn{2}{c}{$\dot{\gamma}\left(\mathrm{rad} \mathrm{s}^{-1}\right)$} \\
\cline { 3 - 5 } & & & $h=100{ }^{\circ} \mathrm{C} \mathrm{Myr}^{-1}$ & $d r / d t=3.9 \mathrm{~mm} \mathrm{Myr}$ \\
\hline CT252 & 46 & 398 & $9.59 \times 10^{-12}$ & $1.15 \times 10^{-13}$ \\
CT262 & 45.30 & 354 & $8.66 \times 10^{-12}$ & $1.03 \times 10^{-13}$ \\
CT253 & 45.30 & 260 & $6.36 \times 10^{-12}$ & $7.56 \times 10^{-14}$ \\
CT241 & 39.96 & 564 & $1.59 \times 10^{-11}$ & $1.67 \times 10^{-13}$ \\
CT246 & 37.00 & 599 & $1.79 \times 10^{-11}$ & $1.74 \times 10^{-13}$ \\
\hline
\end{tabular}

Table 3.2: Matrix phase EPMA (wt.\%) and LA-ICP-MS (ppm) spot analyses. $n$ number of analyses; $\pm 1 \sigma$ standard deviation of the mean. LA-ICP-MS analyses for elements from $\mathrm{Gd}$ to $\mathrm{Hf}$ are excluded because all values are zero.

\begin{tabular}{|c|c|c|c|c|c|c|}
\hline \multirow[b]{2}{*}{$n$} & \multicolumn{2}{|c|}{ Muscovite } & \multicolumn{2}{|c|}{ Chlorite } & \multicolumn{2}{|c|}{ Biotite } \\
\hline & 14 & $\sigma$ & 13 & $\sigma$ & 7 & \\
\hline \multicolumn{7}{|l|}{$(w t \%)$} \\
\hline $\mathrm{SiO} 2$ & 47.70 & 0.12 & 24.83 & 0.05 & 36.19 & 0.11 \\
\hline $\mathrm{TiO} 2$ & 0.25 & 0.01 & 0.09 & n.d. & 1.52 & 0.04 \\
\hline $\mathrm{Al} 2 \mathrm{O} 3$ & 36.20 & 0.15 & 22.31 & 0.13 & 18.14 & 0.08 \\
\hline $\mathrm{FeO}$ & 0.89 & 0.01 & 27.59 & 0.21 & 21.11 & 0.05 \\
\hline $\mathrm{MnO}$ & 0.01 & n.d. & 0.05 & n.d. & 0.03 & n.d. \\
\hline $\mathrm{MgO}$ & 0.58 & 0.03 & 12.27 & 0.11 & 8.43 & 0.04 \\
\hline $\mathrm{CaO}$ & n.d. & n.d. & 0.03 & n.d. & 0.01 & n.d. \\
\hline $\mathrm{Na} 2 \mathrm{O}$ & 1.79 & 0.06 & 0.07 & 0.01 & 0.23 & 0.01 \\
\hline $\mathrm{K} 2 \mathrm{O}$ & 8.63 & 0.10 & 0.60 & 0.02 & 8.67 & 0.02 \\
\hline $\begin{array}{l}\text { Total } \\
(p p m)\end{array}$ & 96.05 & - & 87.84 & - & 94.33 & \\
\hline $\mathrm{Li}$ & 23.05 & 2.38 & 268.26 & 13.64 & 366.70 & 48.65 \\
\hline $\mathrm{Sc}$ & 17.07 & 1.45 & 10.70 & 0.77 & 19.83 & 1.43 \\
\hline V & 162.30 & 1.88 & 104.99 & 5.44 & 208.27 & 19.64 \\
\hline $\mathrm{Cr}$ & 138.13 & 4.39 & 133.88 & 15.56 & 213.76 & 14.04 \\
\hline Co & 5.66 & 1.42 & 147.40 & 6.40 & 107.36 & 11.29 \\
\hline Zn & 44.70 & 10.55 & 976.08 & 47.27 & 655.97 & 69.95 \\
\hline $\mathrm{Ga}$ & 52.51 & 0.75 & 61.45 & 47.27 & 45.51 & 4.86 \\
\hline $\mathrm{Rb}$ & 354.56 & 10.92 & 72.03 & 40.78 & 1969.57 & 188.22 \\
\hline $\mathrm{Sr}$ & 178.86 & 7.14 & 1.45 & 1.01 & 1.91 & 0.31 \\
\hline $\mathrm{Y}$ & 0.02 & 0.01 & 0.01 & 0.01 & 0.02 & 0.01 \\
\hline $\mathrm{Zr}$ & 0.10 & 0.02 & n.d. & n.d. & 0.03 & 0.01 \\
\hline $\mathrm{Nb}$ & 13.85 & 0.27 & 3.18 & 0.99 & 80.46 & 7.83 \\
\hline Cs & 3.99 & 0.47 & 6.54 & 4.16 & 115.71 & 19.78 \\
\hline $\mathrm{Nd}$ & 0.01 & 0.01 & n.d. & n.d. & n.d. & n.d \\
\hline $\mathrm{Sm}$ & n.d. & n.d. & n.d. & n.d. & n.d. & n.d. \\
\hline $\mathrm{Eu}$ & 0.44 & 0.04 & n.d. & n.d. & n.d. & n.d. \\
\hline
\end{tabular}



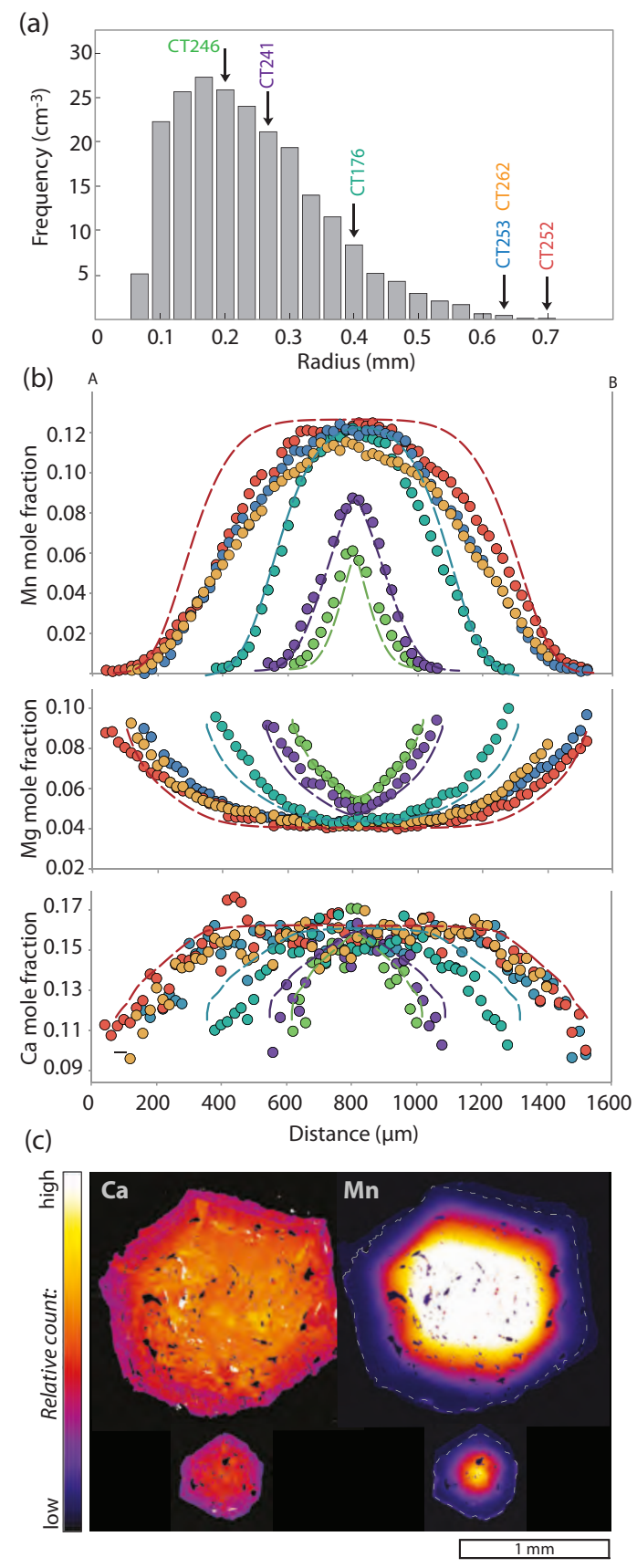

Figure 3.1: (a) Crystal size-distribution for sample 24-99 as determined by XR $\mu$ CT. Arrows indicate bins in which crystals have been mapped using LA-ICP-MS. (b) $\mathrm{Mn}, \mathrm{Mg}$ and Ca EPMA zoning profiles for corresponding mapped garnets. Dashed lines are modelled garnets from George \& Gaidies (2017). (c) Ca and Mn EPMA maps of largest (top) and smallest (bottom) segmented garnets. 


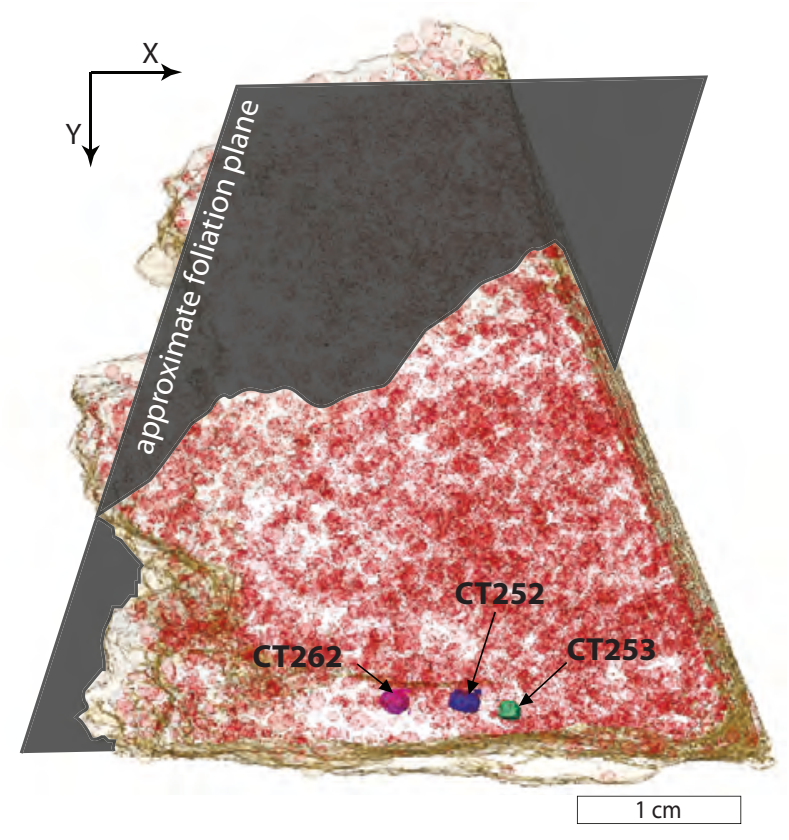

Figure 3.2: $\mathrm{X}-\mathrm{Y}$ view of whole rock volume of sample 24-99 as scanned with $\mu$-CT. The three largest garnets are highlighted (all other garnets in red), and the orientation of approximate foliation plane is illustrated.

Figure 3.3 (following page): Selected quantified LA-ICP-MS raster maps for crystal CT252, the largest centrally sectioned garnet. All maps show ppm abundances with linear, log and ECDF scaling to accentuate zoning. Warmer colours indicate higher concentrations, and for the HREE+Y the maximum scaled value is saturated, such that the highest concentration exceeds the maximum scaled value. Line A-B shows the location of EPMA major element zoning traverse in Fig. 3.1b, boxes on Y map highlight location of Fig. 3.10. Raster direction is from left to right. 


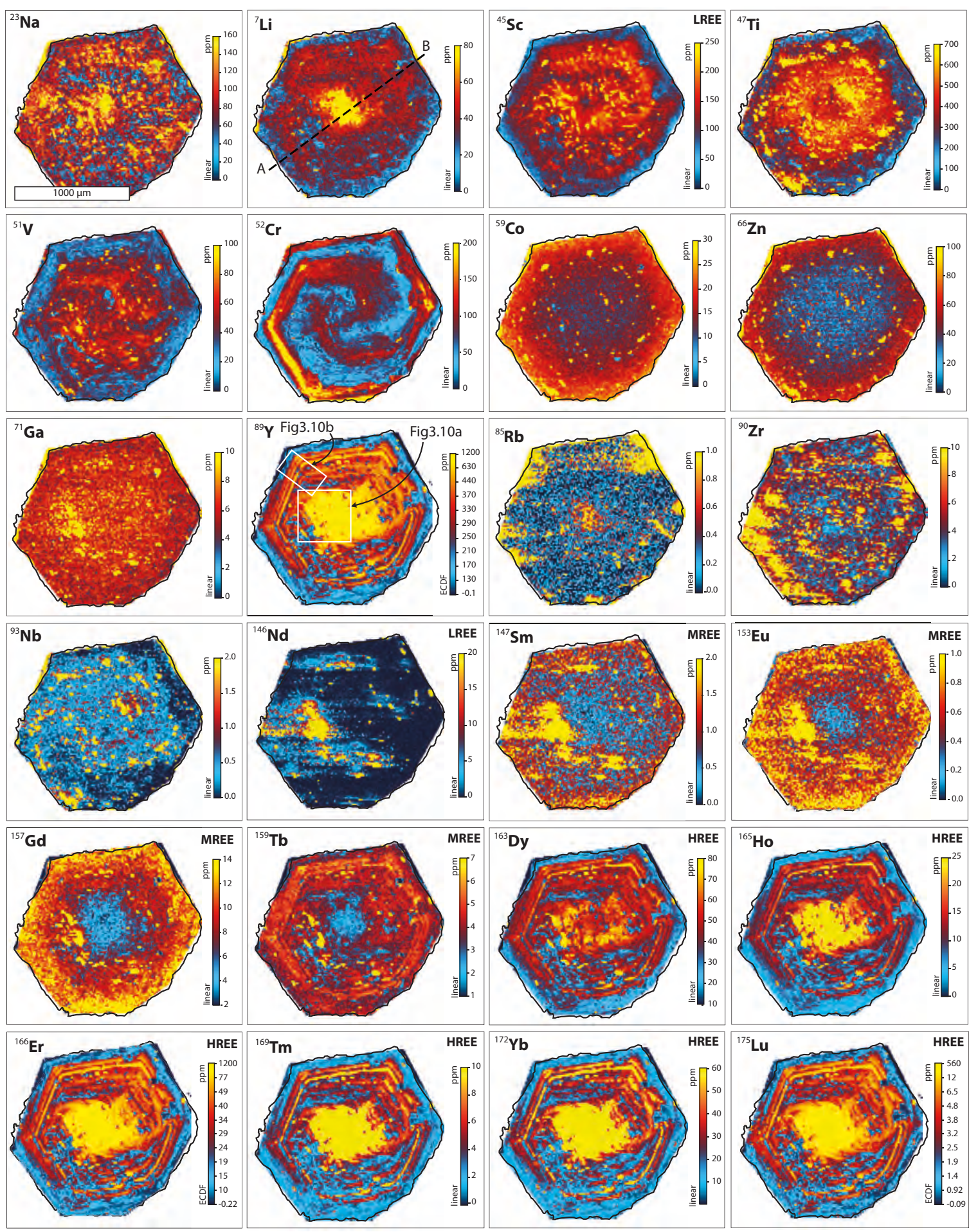



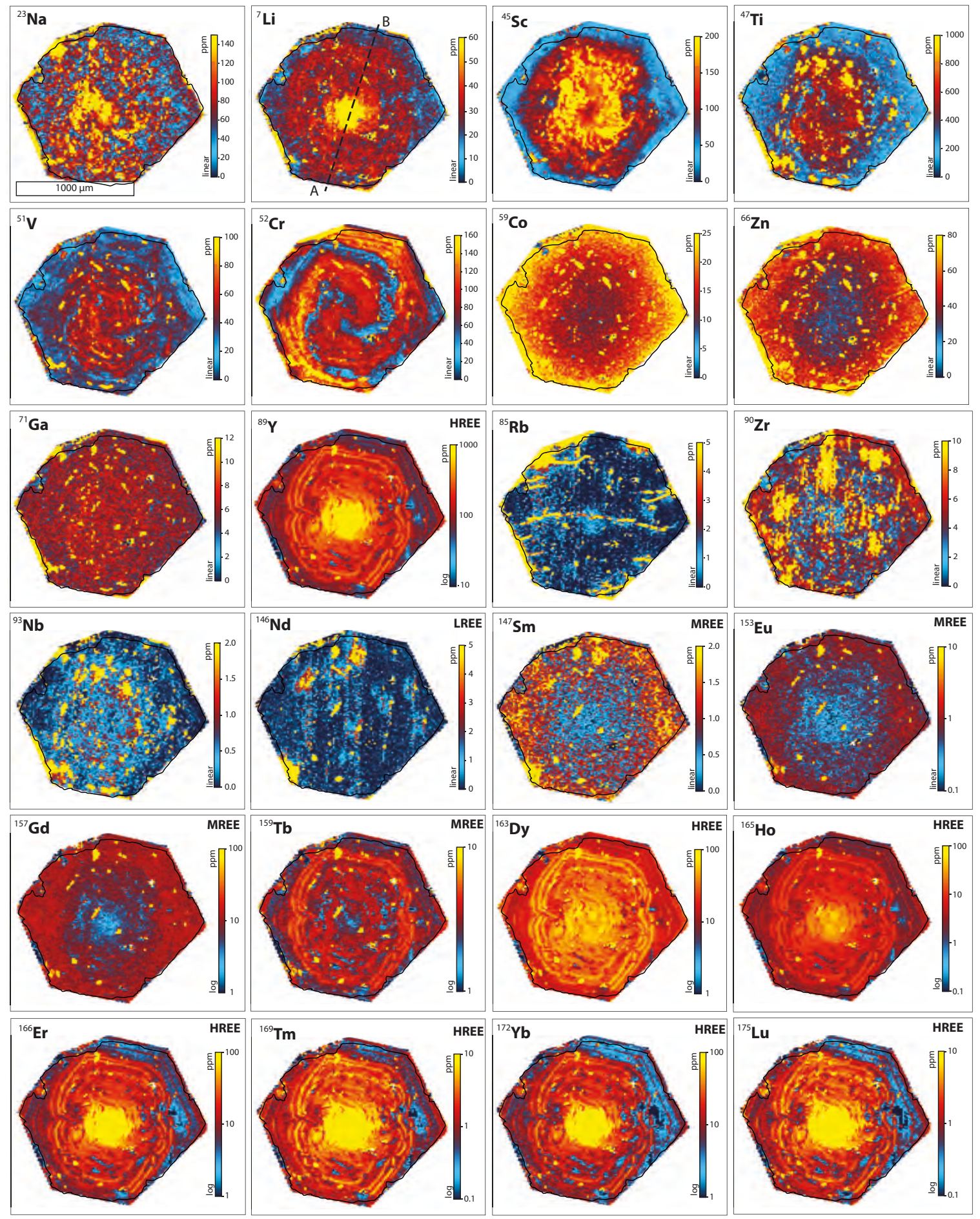

Figure 3.4: As Fig. 3.3 for crystal CT262. Raster direction is from south-southwest to north-northeast. 

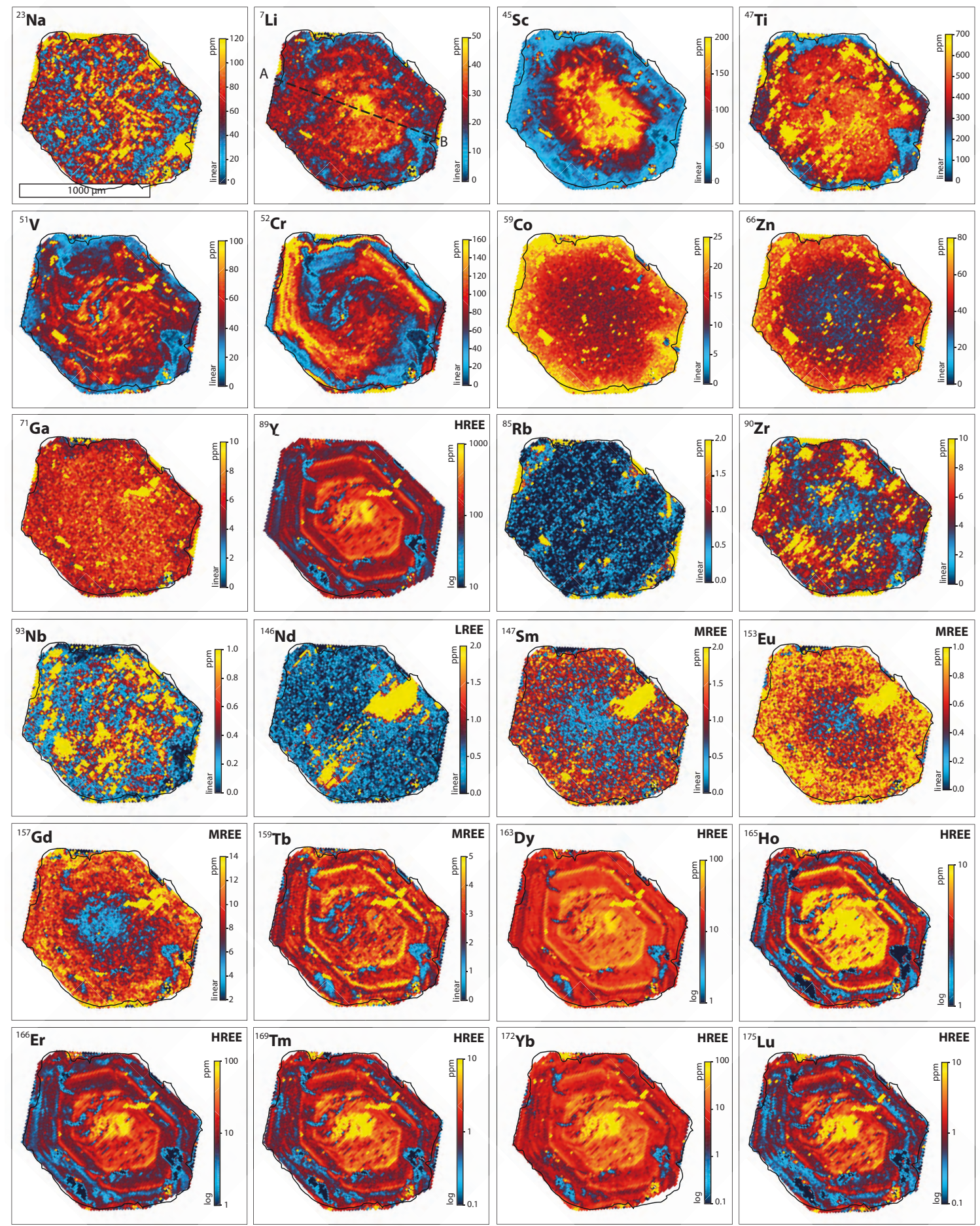

Figure 3.5: As Fig. 3.3 for crystal CT253. Raster direction is from southwest to northeast. 


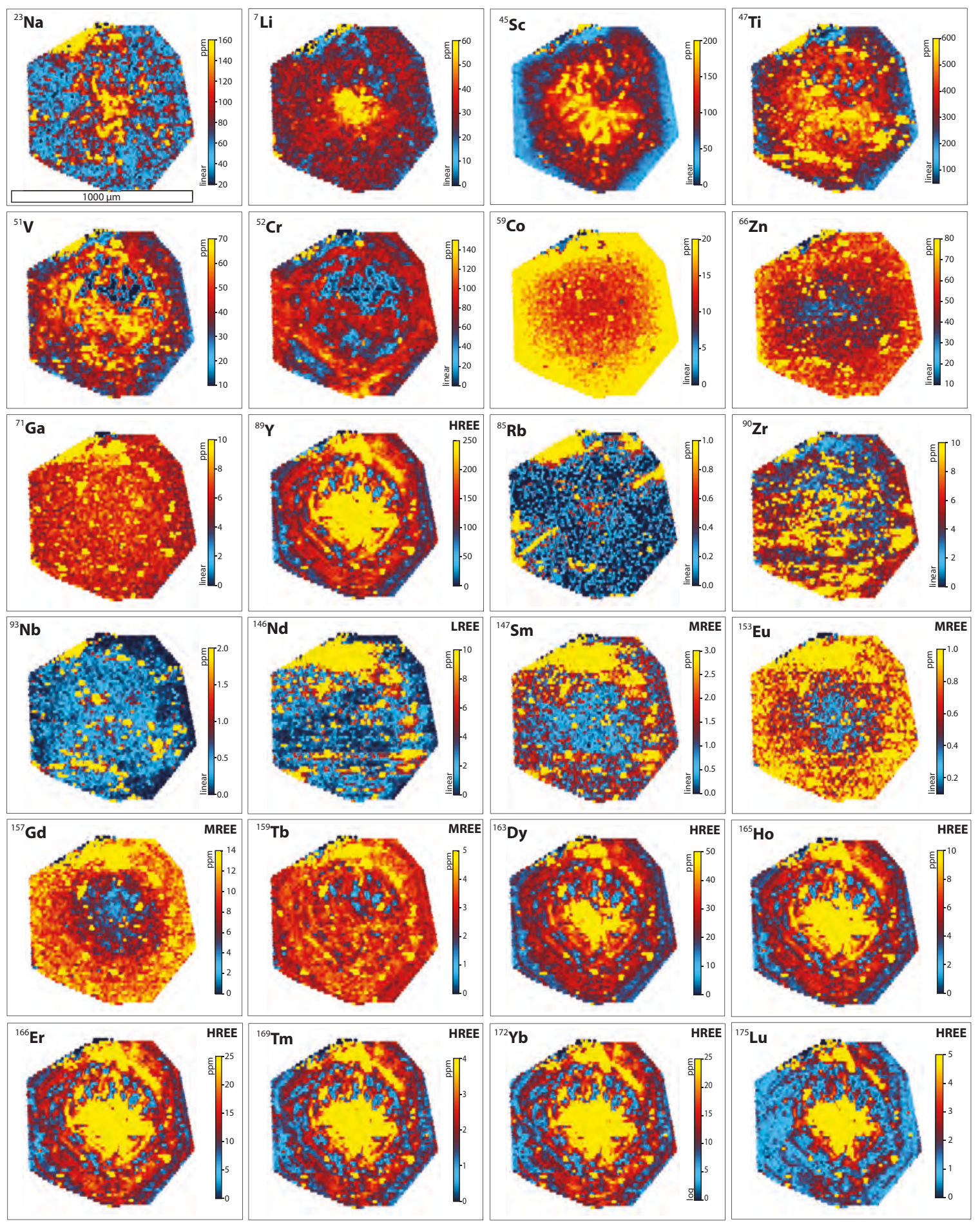

Figure 3.6: As Fig. 3.3 for crystal CT176. Raster direction is from west to east. 

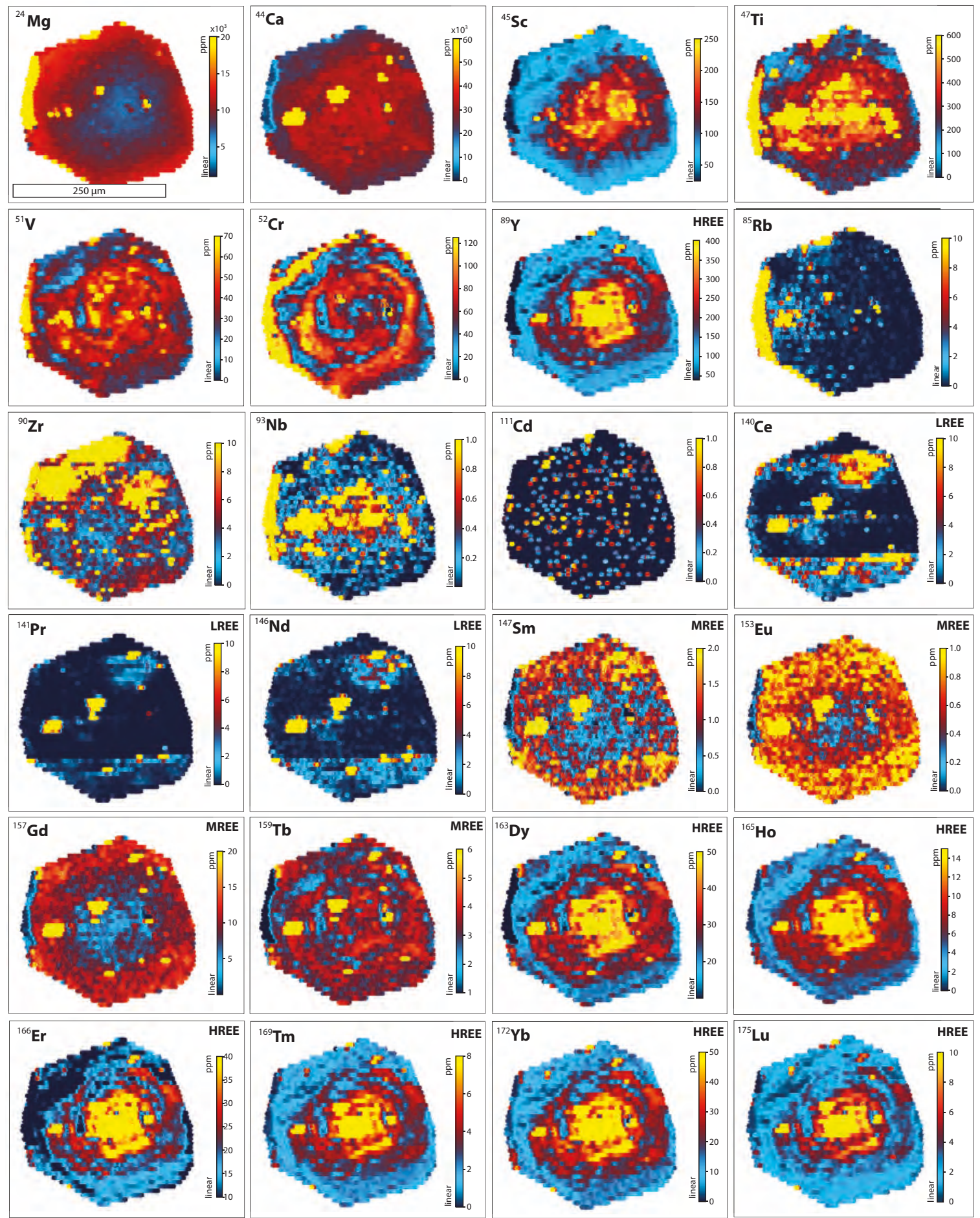

Figure 3.7: As Fig. 3.3 for crystal CT241. Raster direction is from west to east. 


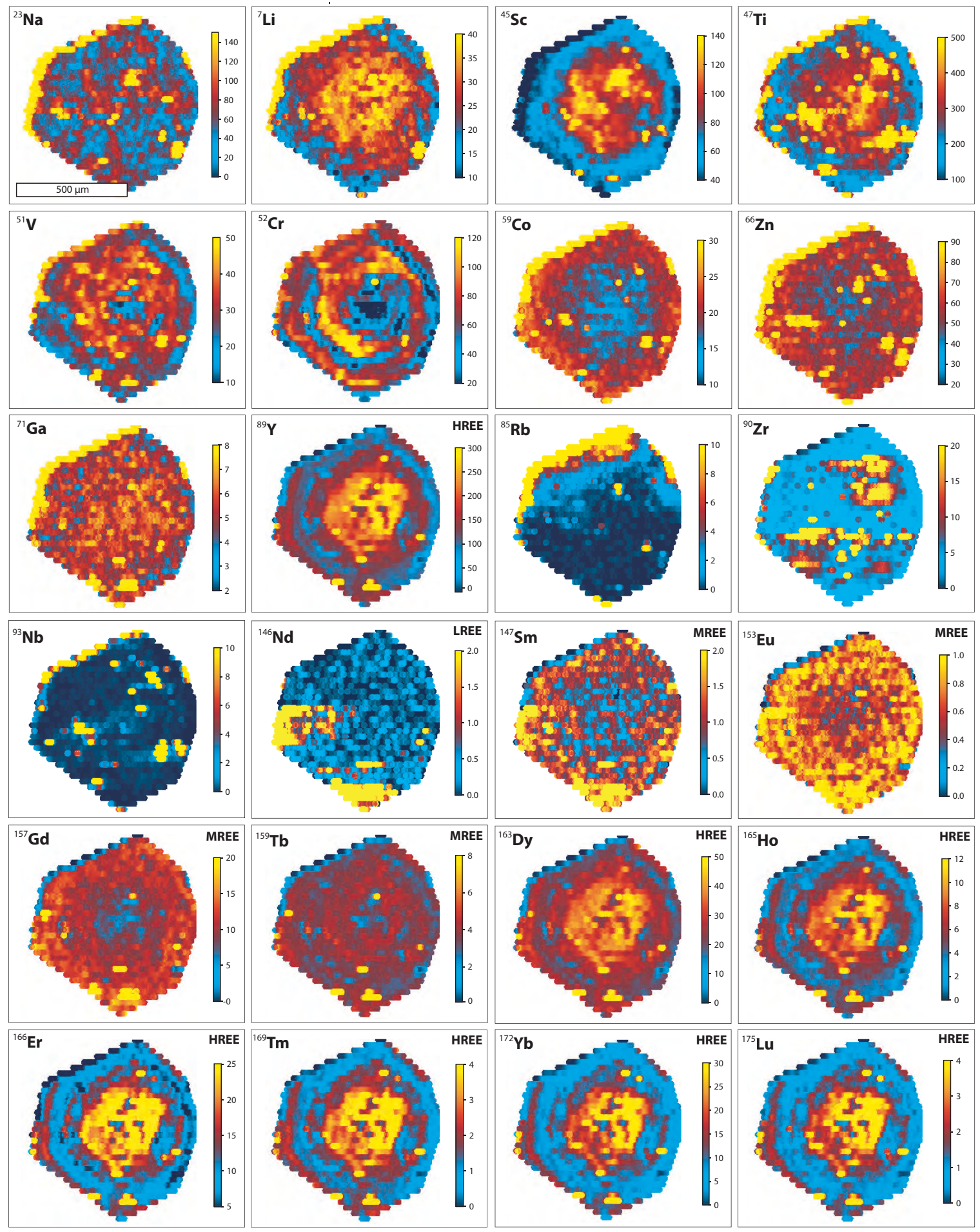

Figure 3.8: As Fig. 3.3 for crystal CT246. Raster direction is from west to east. 
Figure 3.9 (following page): (a) Illustration of key HREE+Y zoning features exhibited by mapped garnets. Red lines denote line along which quantitative (ppm) profiles in (b) to (g) are collected. For each crystal, upper plot contains HREE+Y, and lower plot shows MREE. Note differential y-axis scaling in HREE $+Y$ for differently crystals. Dashed vertical lines highlight alignment of element peaks. 

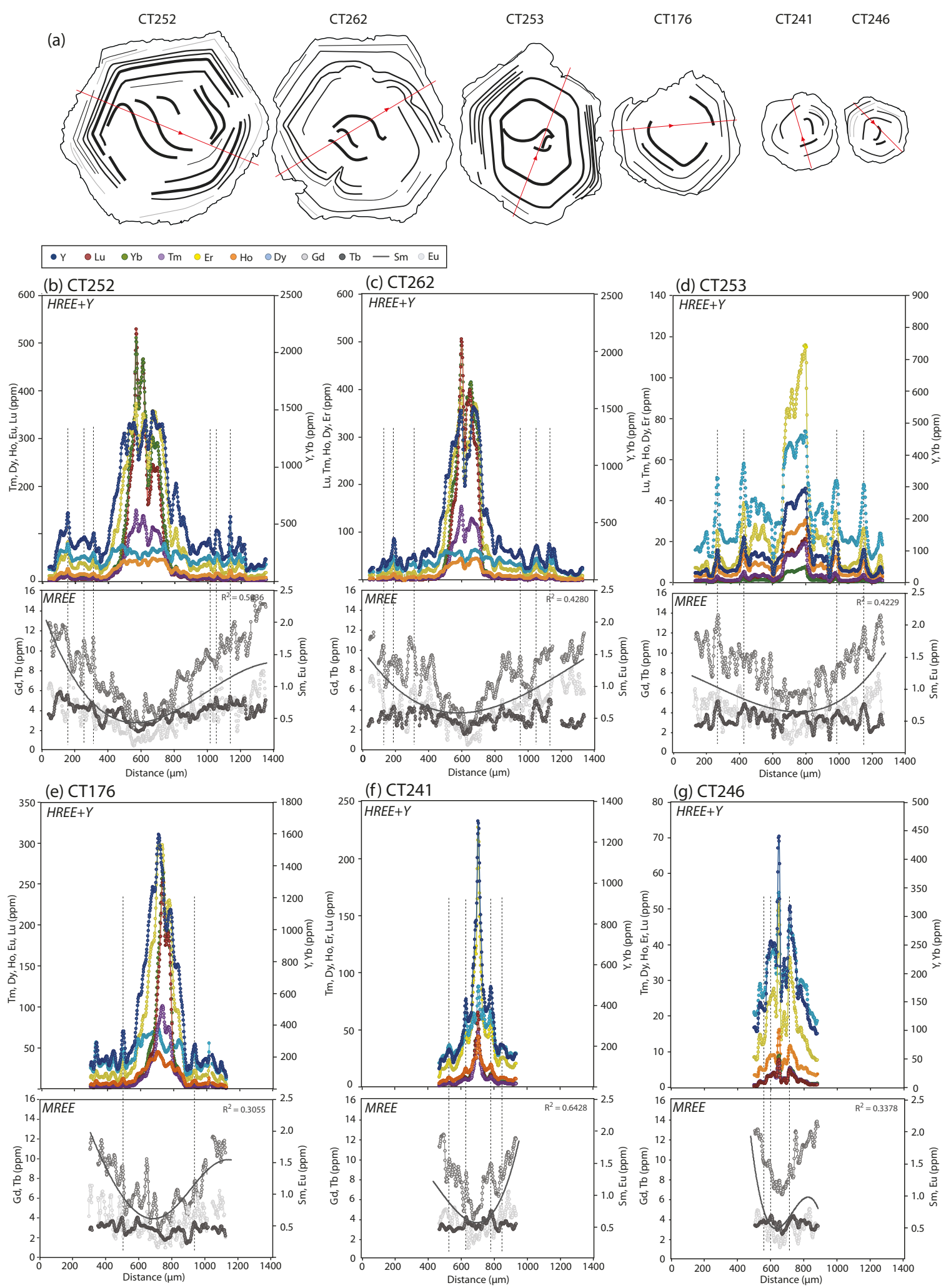


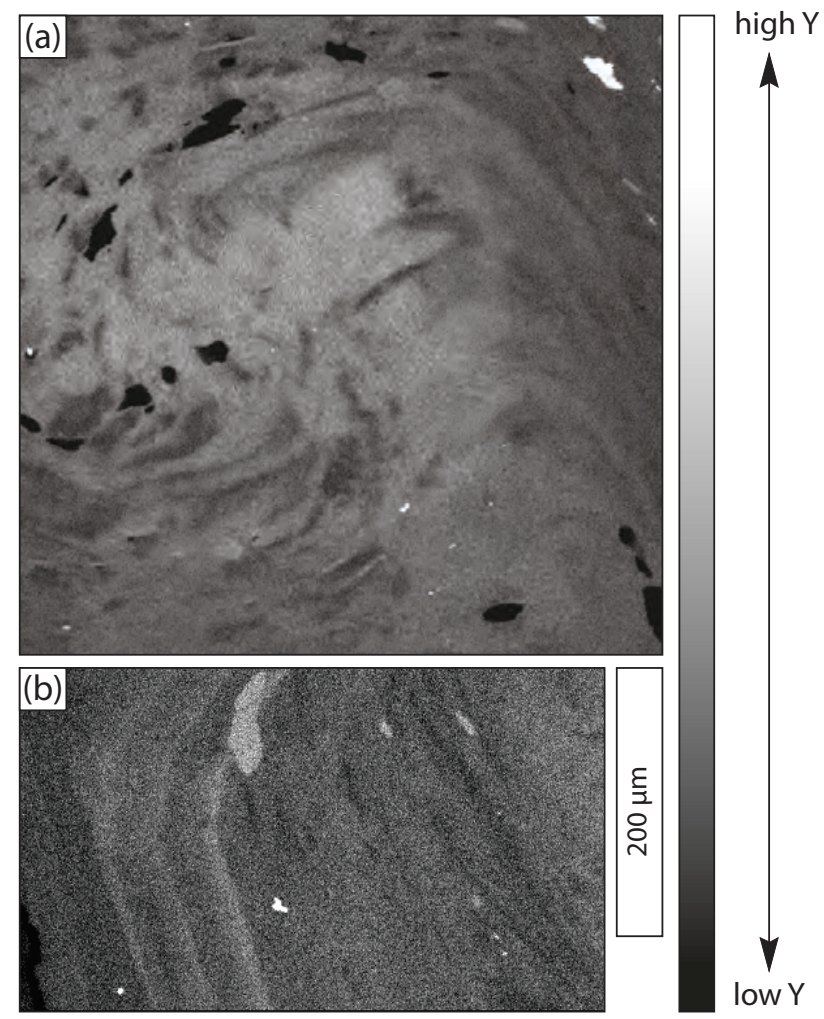

Figure 3.10: FE-EPMA maps of $\mathrm{Y}$ in the core (a) and rim (b) of garnet CT252. Mapped regions highlighted on LA-ICP-MS Y map (Fig. 3.3). Brighter colours denote high concentrations, dark colours indicate lower concentrations. 

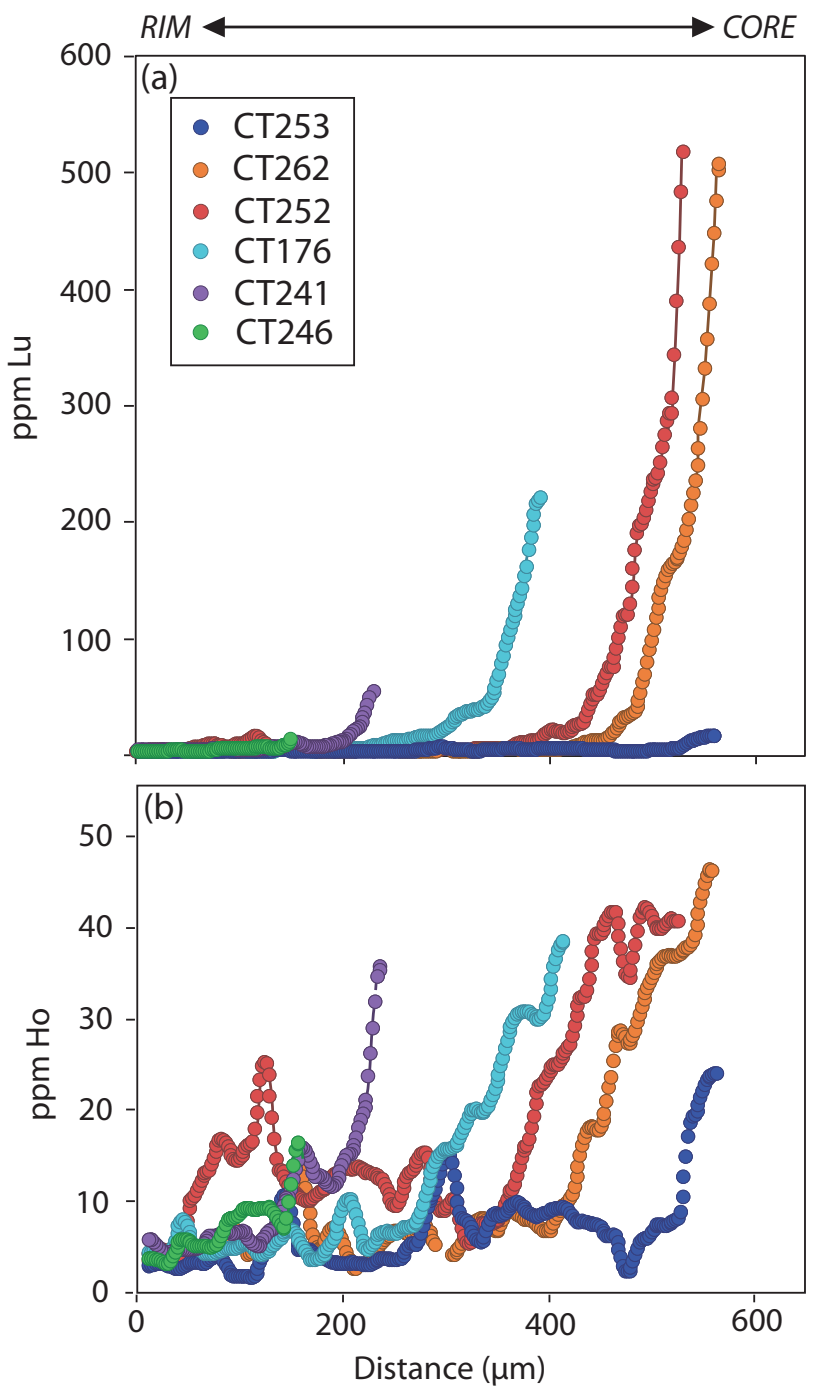

Figure 3.11: Lu (a) and Ho (b) concentrations plotted against distance from rim for the mapped garnets. Core-rim concentration differences are larger for the heaviest REE (with the exception of CT253, see text for discussion). 

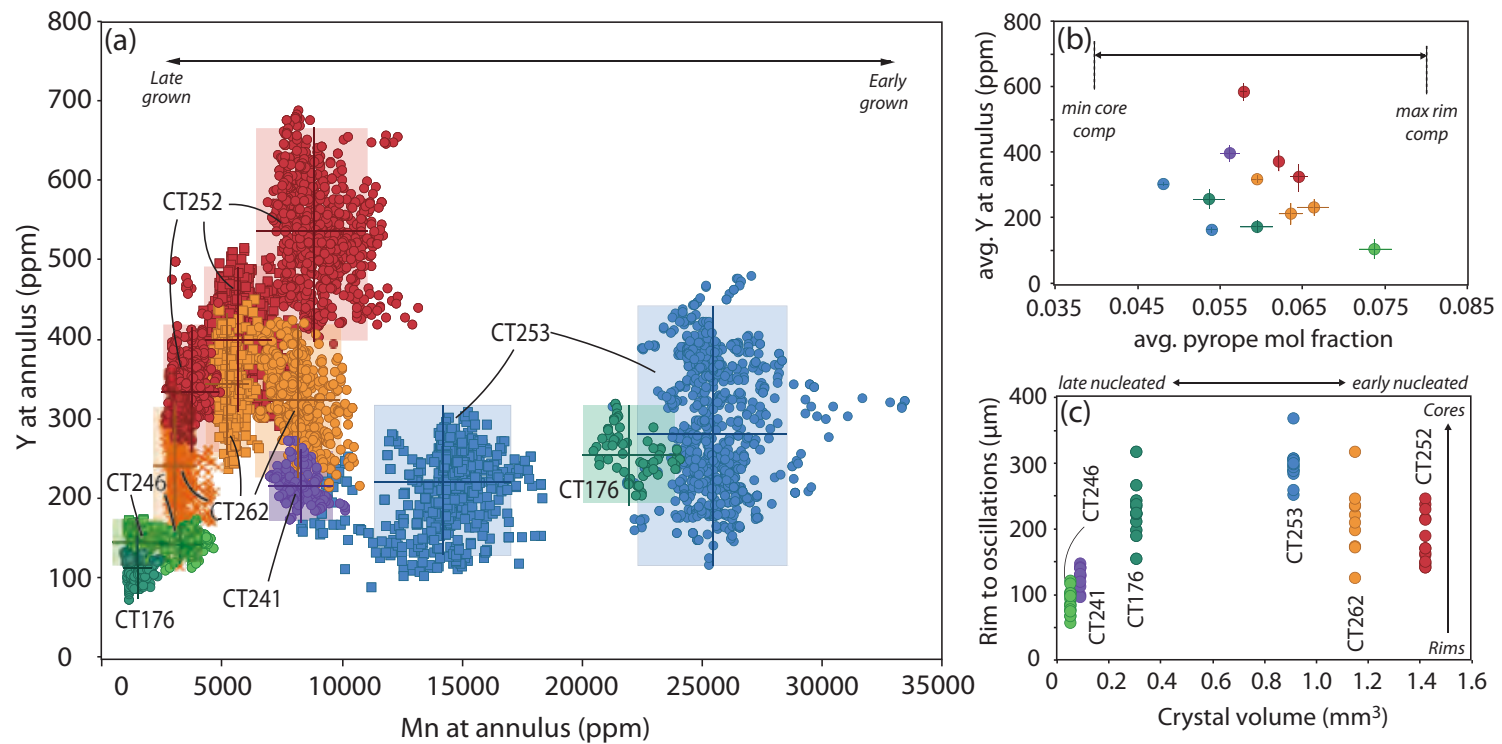

Figure 3.12: (a) Mn concentration versus $\mathrm{Y}$ concentration along garnet annuli. Average and $\pm 2 \sigma$ values for each annuli plotted as crosses. (b) Pyrope fraction versus $\mathrm{Y}$ concentration at annuli. Each point corresponds to averaged composition of LHS and RHS along major rim-to-rim profiles A-B, in Fig. 3.1b, with absolute variation plotted. (c) Measured distance from garnet rims to point at which HREE zoning becomes concentric, compared to absolute crystal volume (as measured with $\mathrm{XR}-\mu \mathrm{CT}$ ). Some scatter associated with measurement from numerous dodecahedral surfaces. 


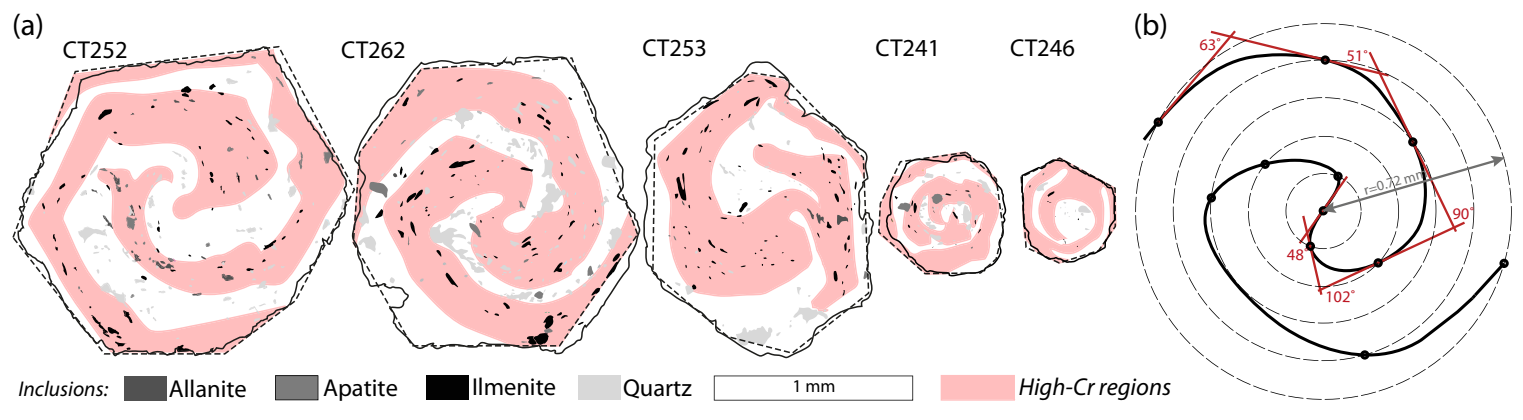

Figure 3.13: (a) Mapped inclusions of allanite, apatite, ilmenite and quartz, in addition to illustration of high-Cr regions in mapped garnets, in red. Solid lines denote edge of crystal and dashed lines indicate extent of LA-ICP-MS map. Garnet CT176 was not mapped with EPMA X-ray mapping, so inclusions cannot be definitively identified. (b) Schematic sketch of spherical approximation of crystal CT262 showing average curvature. Intersects between average curvature and contours (black dots) were used to construct tangents to the curvature and calculate the angle of rotation (in red). 


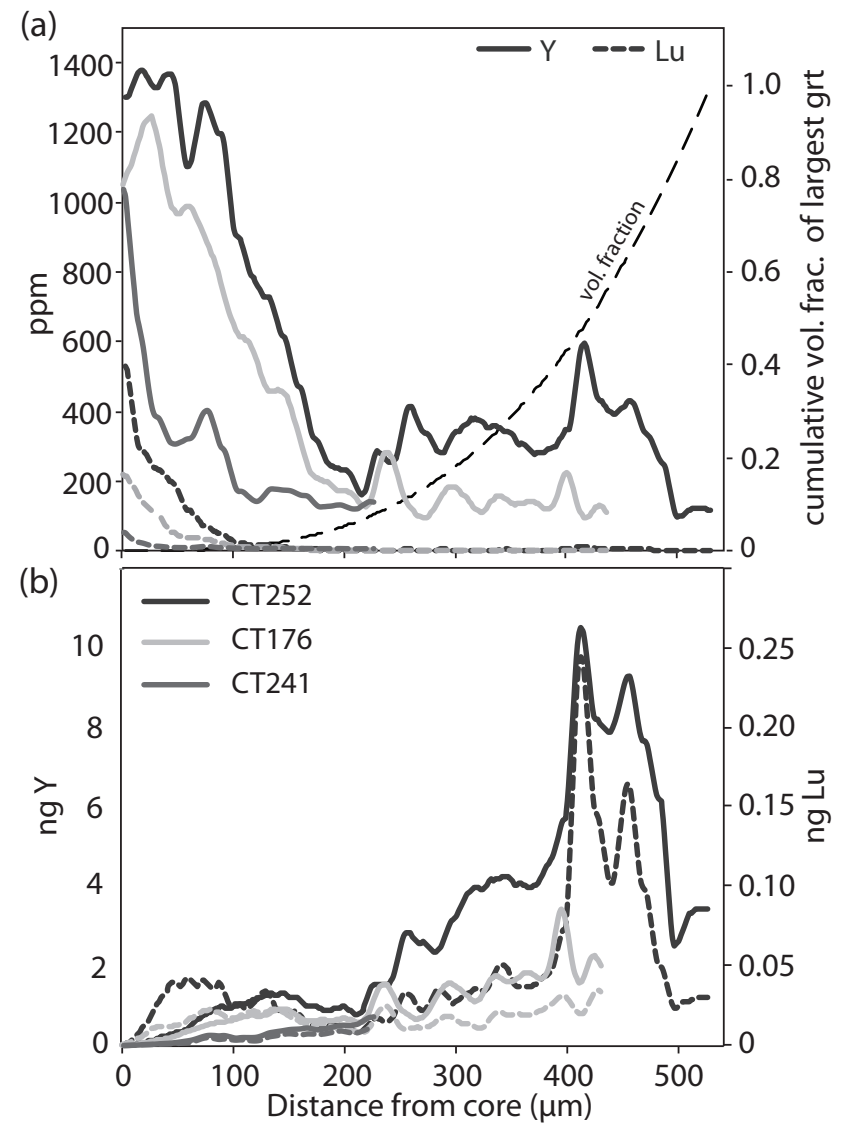

Figure 3.14: (a) Core-to-rim Y and Lu in garnet CT252, CT176 and CT241. Dashed curve is the volume fraction of total garnet CT252 at each radial increment. (b) Concentration profiles from (a) recalculated as a function of the spherical geometry (in ng). Integration was conducted by dividing shells of compositionally homogeneous garnet by a $2 \mu \mathrm{m}$ radial spacing. 


\section{Chapter 4}

\section{Porphyroblast microstructure: sequence continuity and controls on irregular morphologies}

\subsection{Introduction}

Since the early works of Spry (1969) and Cashman \& Ferry (1988), it has been widely accepted that porphyroblastic textures vary systematically with metamorphic grade: samples that experienced lower peak pressure-temperature $(P-T)$ conditions generally contain smaller but more abundant porphyroblasts compared to their high grade equivalents, where there is a tendency for larger, but fewer porphyroblasts. This relationship is an approximate one - as porphyroblast size and abundance can be seen to vary in any given outcrop - and detailed microstructural study has indicated that it may not always be present along a metamorphic field gradient (Hirsch, 2007).

Uncertainty surrounds the cause of this trend, and one hypothesis (Carlson, 1999) ascribes it to sluggish kinetics at low- $T$, which inhibit reaction until large numbers of closely-spaced nuclei form and grow to small crystal sizes, producing an inverse correlation of crystal size with density. Alternatively, theories of Ostwald ripening (e.g., Lifshitz \& Slyozov, 1961; Cashman \& Ferry, 1988; Miyazaki, 1991, 2000) postulate that at high $T$ s larger crystals grow at the expense of small ones. Based on ther- 
modynamic considerations, the stable garnet volume in a regionally metamorphosed average metapelite can be predicted to increase along the prograde path until the stabilisation of staurolite-bearing assemblages, with renewed garnet growth only after destabilisation of staurolite at higher- $T$. However, thermodynamic considerations alone cannot account for porphyroblastic texture and needs to be linked with the kinetics of the underlying processes in order to understand the distribution of volume on individual porphyroblasts.

More generally, the kinetics and energetics of porphyroblast-forming reactions will be strongly influenced by changing extrinsic variables (e.g., Carlson, 1989; Fisher, 1978; Kretz, 1974), as will the diffusive connectivity through the grain boundary network (e.g., Carlson \& Gordon, 2004). The degree of pre-, syn- and post-crystallisation strain (i.e., deformation) may serve to influence porphyroblast texture by providing favourable nucleation sites, reducing grain sizes by recrystallisation, and facilitating grain growth by enhancing kinetics (e.g., Drury \& Urai, 1990). Other factors, such as the inhomogeneity in the distribution of nucleation sites or the spatiallyand temporally-variable distribution of components required for metamorphic mineral growth can influence the development of porphyroblastic texture, and can suppress the development of systematic texture-grade trends (c.f. Ketcham, 2005).

Garnet populations in metapelitic samples from the inverted Barrovian sequence in Sikkim exhibit one of two textural end-members: 'classically' porphyroblastic with isolated garnet porphyroblasts that vary with respect to their size and abundance over changing metamorphic grade, and subordinate garnet-staurolite grade samples that contain highly coalesced and polycrystalline garnet clusters with irregular porphyroblast morphologies. By coupling microstructural assessments with thermobarometric constraints on the $P-T$ evolution, this study aims firstly to assess the continuity of the inverted metamorphic sequence, a subject of continuing debate (e.g., Catlos et al., 
2004; Dasgupta et al., 2004, Bhattacharyya \& Mitra, 2009), and secondly whether the size/abundance-grade trend can be related to systematic variation in the relative $P-T$ histories across the sequence. Post-kinematic coalesced populations enable the empirical investigation of the variables and processes that may have controlled the spatially heterogeneous nucleation and irregular growth of garnet.

\subsection{Geological setting \& sample locations}

The Himalayan orogen, which formed as a result of northward motion of the Indian plate and associated crustal shortening beginning at 55-50 Ma de Sigoyer et al. 2000), exposes one of the worlds most extensive Barrovian inverted metamorphic sequence (IMS), where metamorphic grade and age increases towards progressively shallower structural levels (Mohan et al., 1989, Dasgupta et al., 2004; Anczkiewicz et al., 2014). The Himalayan IMS developed during ductile thrusting along the $2500 \mathrm{~km}$-long Main Central Thrust (MCT, e.g., Sinha-Roy, 1982, Catlos et al., 2001; Yakymchuk \& Godin, 2012), and is particularly well-preserved in the Darjeeling hills and Sikkim (collectively known as the Sikkim Himalaya, Fig. 4.1).

Best developed in the Paleoproterozoic Daling group of the LHS, the IMS can be mapped from below the garnet zone to high grade anatextic conditions in the Greater Himalayan Sequence (GHS) across several transects in Sikkim. However, much debate surrounds its metamorphic and structural continuity, given the variable nature of motion and uncertain location of the MCT zone. Thus, numerous authors have broadly defined the MCT zone and associated deformation in the LHS in different ways, with four predominant schools of thought: (1) as a tectonically imbricated stack with several named thrusts and widespread MCT zone duplexing throughout the IMS (e.g., Robinson et al., 2003; Catlos et al., 2004; Dubey et al., 2005; Bhattacharyya 
\& Mitra, 2009); (2) more localised tectonic interleaving over $\sim 5 \mathrm{~km}$ between the sillimanite-zone of the LHS and the GHS, above an otherwise continuous LHS (e.g., Mottram et al, 2014), (3) as a zone of more limited but pervasive deformation at the top of the IMS which exhibits a continuous metamorphic field gradient from biotite to sillimanite-K felspar grade (e.g., Neogi et al., 1998, Dasgupta et al., 2004; Dasgupta et al., 2009, Gaidies et al., 2015; Chakraborty et al., 2016), and (4) at the base of the IMS (Searle \& Szulc, 2005).

The northernmost transect of the IMS (Fig. 4.1p) is the least condensed and best exposed in Sikkim, and is the focus of this study. Samples were collected along a relatively continuous $10 \mathrm{~km}$ section, from latitude-longitude coordinates $27^{\circ} 26^{\prime} 38^{\prime \prime}$ and $88^{\circ} 31^{\prime} 28.18^{\prime \prime}$ to $27^{\circ} 30^{\prime} 57.8^{\prime \prime}$ and $88^{\circ} 33^{\prime} 27.84$." By focusing in detail on a single transect, diachroneity in development of the sequence parallel to the MCT is excluded as a variable.

\subsection{Analytical methods}

\subsubsection{X-Ray $\mu$-computed tomography}

The size, abundance and shape of minerals in each studied sample were constrained in 3D using a SkyScan 1173 high-resolution X-ray $\mu$-computed tomography (XR $\mu$-CT) scanner at Carleton University, Ottawa, Canada. Scanning was conducted using a $130 \mathrm{kV} / 61 \mu \mathrm{A}$ X-ray source and a $0.25 \mathrm{~mm}$-thick brass filter, with an exposure time of $1300 \mathrm{~ms}$. To fully image the samples but ensure time-efficiency, each was rotated in the conical X-ray beam with a step increment between $0.25^{\circ}$ and $0.30^{\circ}$ over a total $180^{\circ}$. Two-dimensional (2D) attenuation slices were calculated, with the thickness of slices corresponding to between $13.1 \mu \mathrm{m}$ and $48.4 \mu \mathrm{m}$, depending on the setup and size of the hand sample. CT datasets were rendered into 3D X-ray attenuation 
datasets using SkyScan NRecon software with a voxel edge size corresponding to the given slice thickness, and were subsequently processed using the AvizoFire software. Blob3D (Ketcham, 2005) was also used to numerically constrain the locations, sizes, and abundances of garnet crystals in each sample. The largest garnets were identified in the reconstruction, and were isolated and mounted into epoxy mounts to enable central sectioning and analysis with EPMA. Reconstructions were also used to aid in the selection of the most appropriate regions of non-dodecahedral garnet for chemical and crystallographic investigation.

\subsubsection{Whole-rock and mineral major element chemical analyses}

Whole-rock chemical compositions were obtained at Activation Laboratories (Ancaster, Ontario, Canada) using major element fusion inductively coupled plasmaoptical emission spectrometry (ICP-OES), and at the University of Ottawa using wavelength-dispersive X-ray fluorescence analysis (WDXRFA) with a Phillips PW 2400 instrument. For each sample, a rock volume of at least fist-size was crushed and powdered in order to account for any sample-scale heterogeneities or systematic layering in the rock texture. With increasing textural heterogeneity and grain size, crushed volumes were larger. Sample MAN22 contains relatively large porphyroblasts so major elements were not determined using a bulk approach, given the large sample size needed to constrain a representative rock volume. Instead, major element chemistry was determined by combining the average chemical compositions of representative phases with their modal abundances in three thin sections representative of a $150 \mathrm{~cm}^{3} \mathrm{XR} \mu$-CT scan.

Point analyses and compositional maps of major elements were obtained on a JEOL 8230 SuperProbe at the University of Ottawa. Points utilized a $20 \mathrm{kV}$ beam voltage and a current of $20 \mathrm{nA}$ with a peak counting time of $20 \mathrm{~s}$ for all elements. Cal- 
ibration was to a range of natural and synthetic standards, and counts were corrected to elemental wt.\% by the ZAF matrix correction procedure. Unknowns and standard intensities were corrected for deadtime, and standards corrected for instrument drift. Major element maps were produced using a focused acceleration voltage at $20 \mathrm{kV}$, a beam current of $100 \mathrm{nA}$, and a beam diameter of $4-10 \mu \mathrm{m}$.

\subsubsection{Electron backscatter diffraction}

The crystallographic orientations of garnet (and some ilmenite, plagioclase, and quartz) in sample SIM3 were analysed using EBSD. The surface of a standard polished thin section containing garnet structures of interest was mechanically polished down to a final $0.05 \mu \mathrm{m}$ diamond abrasive for $\sim 1$ hour. EBSD data were acquired and automatically indexed using Oxford Instruments AztecHKL software on an FEI Quanta 650 field-emission gun SEM equipped with an Oxford Instruments Nordlys S EBSD camera in the Department of Earth Science at the University of Oxford, United Kingdom. Automatic beam scans were collected at a $70^{\circ}$ thin section tilt and 5 or $8 \mu \mathrm{m}$ step size. Beam conditions for all EBSD data used a $30 \mathrm{kV}$ acceleration voltage and low vacuum conditions, precluding the need for sample coating.

The Matlab toolbox MTEX (Hielscher \& Schaeben, 2008; Bachmann et al., 2010; Mainprice et al., 2011) was used for subsequent data processing, EBSD map construction and pole figure plotting. Data were post-processed by removing 'wild spikes' (isolated measurement points that differ significantly from adjacent measurements, usually a result of sample damage that causes poor pattern generation), and by extrapolating zero solutions based on four neighbours. Good diffraction patterns were obtained for garnet and coarse ilmenite. Quartz was variably indexed, and phyllosilicates were generally unindexable. Quoted misorientations are the minimum angular rotation around some misorientation axis required to rotate a lattice into coincidence 
with an adjacent lattice (Wheeler et al., 2001). Grain boundaries are defined as misorientations exceeding $10^{\circ}$, and subgrain boundaries are misorientations exceeding $1^{\circ}$

\subsubsection{Metamorphic phase equilibria modelling}

To constrain the conditions of metamorphism and porphyroblast crystallisation, phase equilibria modelling has been conducted using the Gibbs energy minimisation software Theriak/Domino de Capitani \& Brown, 1987, de Capitani \& Petrakakis, 2010). Modelling was conducted in the 11-component system $\mathrm{MnO}-\mathrm{Na}_{2} \mathrm{O}-\mathrm{CaO}-\mathrm{K}_{2} \mathrm{O}-\mathrm{FeO}-$ $\mathrm{MgO}-\mathrm{Al}_{2} \mathrm{O}_{3}-\mathrm{SiO}_{2}-\mathrm{CO}_{2}-\mathrm{H}_{2} \mathrm{O}-\mathrm{TiO}_{2}$, using the bulk rock compositions given in Table 4.2. Activity-composition $(a-X)$ relationships of the end-member phases are as described in George \& Gaidies (2017). Metamorphic fluids were modelled as a carbon-, oxygen-, and hydrogen-bearing $(\mathrm{COH})$ phase produced by the equilibration of $\mathrm{H}_{2} \mathrm{O}$ and excess graphite, according to the method presented by Connolly \& Cesare (1993). In addition to graphite saturation, the modelled systems were considered to be saturated with respect to $\mathrm{SiO}_{2}$ and $\mathrm{H}_{2} \mathrm{O}$.

Because growth of chemically zoned garnet is likely to have fractionated the bulk chemistry, core isopleth thermobarometry has been coupled with forward garnet crystallisation modelling for key samples using the THERIA_G software Gaidies et al., 2008) to constrain the $P-T$ conditions over the duration of garnet growth. The same thermodynamic database as used for calculation of phase equilibria was used for this modelling, and kinetic parameters of Chakraborty \& Ganguly (1992) were used to account for intragranular diffusion of $\mathrm{Mn}, \mathrm{Mg}$ and Fe in garnet. The diffusivity of $\mathrm{Ca}$ is half the diffusivity of Fe, following the approach described by Loomis et al. (1985) and Florence \& Spear (1991). The software is fully described in Gaidies et al. (2008) and George \& Gaidies (2017), and is not outlined here. 


\subsection{Petrographic and chemical characterisation of samples}

Figure 4.2 shows representative XR $\mu$-CT scans and garnet crystal size distributions (CSDs) of select samples from the northern IMS, with corresponding textural parameters presented in Table 4.1. A general increase in garnet porphyroblast size with increasing grade is coupled with a corresponding decrease in porphyroblast density, with the exception of the SIM samples, which contain highly impinged garnet populations that could not be segmented. There is no corresponding trend in the overall shape of the CSDs. Table 4.2 contains major element bulk rock chemistry for all samples in Fig. 4.2. Key samples (starred in figures and tables) are representative of overall trends, and are discussed in detail.

\subsubsection{Petrography and mineral chemistry}

Microstructural and chemical characteristics are described below, starting with the lowest grade sample MAN1 and finishing with the highest grade studied sample, MAN22 at the northern end of the traverse. Tables 4.3 and 4.44 .5 contain modal mineral proportions and representative EPMA analyses, respectively. Modal proportions are determined by integrating random point counting in several thin sections, optical petrography and XR $\mu$-CT greyscale thresholding. Chemical analyses are presented as the average of multiple grains, and multiple averages are presented for phases that exhibit significant textural variation. Garnet chemistries were determined with EPMA point analysis across geometrically central sections (Fig. 4.4, element mapping (Fig. 4.6) and compositional traverses across non-central sections (Fig. 4.7). 


\section{MAN1}

MAN1 is fine-grained and continuous, with $\mathrm{S}_{1}$ folded into a weak $\mathrm{S}_{2}$ matrix crenulation comprising muscovite, biotite, chlorite and ilmenite, with interstitial quartz which exhibits a slight shape-preferred orientation that is evenly disseminated throughout the matrix (Fig. $4.3 \mathrm{a} \& \mathrm{~b}$ ). Matrix micas are generally $<100 \mu \mathrm{m}$ long, although some biotite is $\sim 500 \mu \mathrm{m}$ long. The paragonite content of muscovitic white mica $\left[X_{P a}=\right.$ $\mathrm{Na} /(\mathrm{Na}+\mathrm{K}+\mathrm{Ca}) \times 100]$ varies from $10 \%-20 \%$, and biotite has $X_{\mathrm{Mg}}[\mathrm{Mg} /(\mathrm{Mg}+$ $\left.\left.F e^{2+}\right)\right]$ of $0.33-0.40$ and $\sim 0.1$ cpfu Ti. The chemical composition of matrix phases does not vary systematically throughout the analysed sections. Quartz grain sizes vary from 75 to $230 \mu \mathrm{m}$, and biotite pinning quartz grain boundaries has equivalent chemistry to that intergrown with muscovite. Scarce plagioclase has been identified in thin section (Table 4.3).

$\mathrm{S}_{2}$ is deflected by subhedral to anhedral garnet, which is commonly mantled by thick (50-150 $\mu \mathrm{m})$ retrograde chlorite strain caps (with $X_{\mathrm{Mg}}=0.39-0.40$ ) that parallel $\mathrm{S}_{2}$. Ilmenite inclusions can still be seen to be continuous with the matrix (Fig. $4.3 \mathrm{~b}$ ), indicating that deformation was unlikely to have significantly outlasted growth. Quartz pressure shadows around garnet are common. Spiral quartz and ilmenite in-

clusion trails dominate the core regions of garnet, suggesting that garnet-core growth was syn-kinematic with respect to foliation-forming deformation. Garnet cores also contain clinozoisite, apatite and allanite.

XR- $\mu$ CT scanning (Fig. 4.2a) reveals maximum and minimum sphere-normalised garnet radii of 0.84 and $0.08 \mathrm{~mm}$, respectively, and a mean garnet radius of $0.38 \mathrm{~mm}$ (Table 4.1). These radii do not consider the volume of garnet replaced by chlorite, implying that the mean garnet radius will have been slightly elevated at peak conditions relative to that now measured. The garnets have a crystal density of 169 garnets $\mathrm{cm}^{-3}$ and make up $\sim 3$ vol. $\%$ of the scanned rock volume. They comprise a 
positively-skewed CSD, with a skewness of 0.19, though there is some slight layering parallel to the foliation with respect to garnet crystal size.

The largest garnet in the scanned volume preserves steep-sided prograde compositional zoning for all garnet end-member components (Fig. 4.4 a). Core regions contain spessartine $\left(X_{\mathrm{sps}}\left[=M n /\left(F e^{2+}+C a+M g+M n\right)\right]\right)$ of $\sim 19$ mol.\%, decreasing to $<1 \mathrm{~mol} . \%$ in garnet rims. Almandine $\left(X_{\mathrm{alm}}\left[=F e^{2+} /\left(F e^{2+}+C a+M g+M n\right)\right]\right)$ and grossular $\left(X_{\mathrm{grs}}\left[=\mathrm{Ca} /\left(\mathrm{Fe}^{2+}+\mathrm{Ca}+\mathrm{Mg}+\mathrm{Mn}\right)\right]\right)$ contents increase and decrease from core-to-rim, from 58 to c. $80 \mathrm{~mol} \%$ and c. 19 to $12 \mathrm{~mol} \%$, respectively. At the transition from inclusion-rich core to inclusion-free rim, the almandine and pyrope $\left(X_{\text {prp }}\left[=M g /\left(F e^{2+}+C a+M g+M n\right)\right]\right)$ gradients decrease. Pyrope is homogeneous at c. $3 \mathrm{~mol} . \%$ in core regions, and increases over approximately $400 \mu \mathrm{m}$ to $8 \mathrm{~mol} . \%$ at the rims.

\section{4-99}

Sample 24-99 has been extensively described in Anczkiewicz et al. (2014), Gaidies et al. (2015), George \& Gaidies (2017) and George et al. (2018). The population of subdhedral to anhedral garnet is contained within a penetratively foliated $\mathrm{S}_{1}$ matrix comprising white mica, primary chlorite, biotite, quartz and plagioclase, with accessory ilmenite, tourmaline, allanite, apatite and zircon (Fig. 4.3. \& d), which is microfolded to form a weak spaced $\mathrm{S}_{2}$ crenulation cleavage. Micas are on the order of 50-200 $\mu \mathrm{m}$ in length. The chemical composition of minerals are uniform across the assessed region: matrix biotite is not systematically zoned, with $X_{\mathrm{Mg}}$ of $0.41-0.43$ and $0.08-0.10 \mathrm{cpfu} \mathrm{Ti}$, and fine white mica are muscovitic with a paragonite content of $19 \%-28 \%$. Prograde matrix chlorite contains $X_{\mathrm{Mg}}=0.46-0.47$, whereas retrograde

chlorite surrounding garnet and cross-cutting $\mathrm{S}_{2}$ is slightly less Mg-rich, with $X_{\mathrm{Mg}}=$ 0.42-0.44. Plagioclase compositions are not systematically variable throughout the 
thin section, but some grains preserve core-to-rim $\mathrm{Na}$ and $X_{\mathrm{An}}[=\mathrm{Ca} /(\mathrm{Na}+\mathrm{Ca})]$ differences of $0.01-0.05 \mathrm{cpfu}$ and $0.11-0.25$, respectively. $45-500 \mu \mathrm{m}$ quartz is discontinuously present in the matrix with plagioclase.

$\mathrm{XR}-\mu \mathrm{CT}$ characterises a garnet population with a density of $\sim 220$ grains $\mathrm{cm}^{-3}$ that exhibits a lognormal CSD with a skewness of 0.8 (Fig. 4.2 b and Table 4.1). Maximum and minimum sphere-normalised grain radii are $0.71 \mathrm{~mm}$ and $0.053 \mathrm{~mm}$ respectively, and the mean garnet sphere-normalised radius is $0.24 \mathrm{~mm}$. Many crystals exhibit faceted surfaces, with minor retrogression to ragged chlorite. Garnets contain curved-to-sigmoidal inclusion trails comprising aligned ilmenite, apatite, allanite and flattened quartz that is commonly continuous with the external foliation (Fig. $4.3 \mathrm{c}$ \& d), indicating garnet formed synchronously with the final phase of deformation. In the largest garnet (Fig. 4.4p) spessartine content exhibits typical prograde bellshaped zoning, with 17 mol.\% in the core decreasing to zero in the rims. Pyrope and almandine contents are zoned from 5 to $9 \mathrm{~mol} \%$, and 68 to $81 \mathrm{~mol} \%$, respectively, from core to rim. The grossular component broadly decreases from $\sim 16$ mol. $\%$ in the core to $\sim 11$ mol. $\%$ at the rims.

\section{SIM3}

SIM3 is texturally heterogeneous (Figs. 4.5 \& 4.8), and contains microstructures at odds with those in adjacent regions of the IMS. The sample and its microstructure are representative of samples SIM2-5, present along a continuous $\sim 2 \mathrm{~km}$-section of the northern transect (Fig. 4.1). Muscovite (50-200 $\mu \mathrm{m}$ long), minor biotite (c. 50-250 $\mu \mathrm{m}$ long), and abundant foliation-parallel ilmenite define a strongly deformed and anastomosing spaced foliation between 0.3-1 cm-wide quartz-, feldspar-, and minor biotite-bearing $\mathrm{S}_{1}$ microlithons (Fig. $4.5 \mathrm{~d} \& \mathrm{e}$ ). Chlorite has replaced some biotite, and plagioclase associated with quartz is more abundant in this sample than in other 
studied samples (Table 4.3). Accessory tourmaline, apatite and rare rutile are present in the matrix. Coarse biotite is mainly concentrated and aligned with the deformed $\mathrm{S}_{1}$ at the margins of cleavage domains (Fig. 4.5 d), and finer biotite pins quartz-quartz grain boundaries in some layers. Quartz crystal size varies considerably between microlithons, resulting in a significant foliation-parallel grain size layering (Fig. 4.5c).

As can be seen best in the XR- $\mu \mathrm{CT}$ reconstruction (Figs. 4.2 \& $4.5 \mathrm{a}$ ), two distinct garnet varieties broadly define porphyroblastic domains in SIM3: apparently coalesced polycrystalline post-kinematic porphyroblasts that form c. 2-12 mm highlyirregular garnets (Type A garnet) and smaller 50-400 $\mu \mathrm{m}$ isolated subhedral porphyroblastic garnets (Type B garnet). Type A and B garnet together make up 4.6 vol.\% of the total rock volume (c. 5 vol.\% in Type A domains, and c. 2.5 vol. $\%$ in Type B domains). Given the significant coalescence of garnet in this sample which makes segmenting individual garnets in the $\mathrm{XR}-\mu \mathrm{CT}$ dataset unfeasible, garnet CSDs and crystal densities could not be determined.

Type A garnet forms irregular branching clusters that form spatially heterogeneous structures sub-parallel to the orientation of the folded $\mathrm{S}_{1}$ schistosity and which are often present in quartz-rich domains (Fig. 4.5d). Clusters of Type A garnet commonly partially overgrow the interface between microlithons and cleavage domains (Fig. $4.5 \mathrm{~d}-\mathrm{f}$ ), and appear spatially controlled by the location and orientation of mica fabrics surrounded by quartz. Petrographically apparent crystal boundaries within clusters can be identified optically as thin black lines in plane-polarised light, and indicate that Type A garnet clusters comprise numerous coalesced garnet porphyroblasts (hereafter referred to as polycrystals) and coarse grained single porphyroblasts with irregular crystal boundaries (Fig. 4.5p-f).

Abundant inclusions of ilmenite, quartz, apatite and zircon in Type A garnet are aligned and continuous with the anastomosing matrix schistosity (Fig. $4.5 \mathrm{~b} \mathrm{\&} \mathrm{d}-\mathrm{f}$ ), 
and ilmenites transecting the garnet-matrix interface are directly continuous with the external foliation. Polycrystals therefore appear post-kinematic with respect to foliation-forming deformation. Garnet faces in contact with micaceous domains are well developed and euhedral (Fig. 4.5b-f), and are occasionally chloritized. Conversely, where Type A garnet is in contact with quartz \pm plagioclase, the surface is highly irregular to lobate (Fig. 4.5b, d \& e), though is not associated with any secondary or retrograde phase. In places, Type A garnet is skeletal, and locally exhibits mesh and honeycombe textures (with inclusions of quartz), whereby garnet tips preferentially protrude along quartz-quartz interfaces (Fig. 4.5p) and form thin films along grain boundaries. Unlike in garnet-free microlithons, there is negligible biotite pinning quartz grain boundaries in zones surrounding and associated with Type A garnet.

Major element mapping of Type A garnet reveals complex compositional zoning, with numerous chemical maxima in a given structure (Fig. 4.6 a \& b). In the porphyroblast mapped in Fig. 4.6 b, the zoning of $\mathrm{Mn}, \mathrm{Mg}$ and Fe is concentric and continuous about the entire garnet surface. Fe, Mg, and Mn zoning is also broadly concentric about individual internal garnet-garnet porphyroblast boundaries in a polycrystal, and parallel to external garnet-matrix interfaces (Fig. 4.6a). Ca is zoned broadly at the scale of the polycrystal, with decrease in Ca only at external rims. In addition to a patchy overall distribution and polycrystal-scale chemical zoning of grossular, the distribution of Ca exhibits compositional sector zoning along $\{211\}$ planes. The largest mapped porphyroblast in Fig. 4.6a contains c. 7-8 mol.\% spessartine, 6-7 mol.\% pyrope, 9 mol.\% grossular and 76-66 mol.\% almandine in its core (Fig. 4.7 a), with smaller porphyroblasts (generally towards the centre of the structure in Fig. 4.6a) containing less $\mathrm{Mn}$ in their cores. The apparent chemical cores in larger external porphyroblasts are situated towards the interior of the polycrystal, not in the 
morphological centre of each grain (e.g., box, Fig. 4.6a).

Euhedral external rims of garnet polycrystals that are in contact with micaceous domains reach rim minima/maxima that are similar in all porphyroblasts within a polycrystal: c. 0 mol.\% spessartine, 9-11 mol.\% pyrope, $7-11 \mathrm{~mol} . \%$ grossular and 77-83 mol.\% almandine (Figs. 4.6a \& 4.7a-b). These extrema are succeeded by a $<10 \mu \mathrm{m}$ wide rim zone in which Mn values reach an outermost peak of $1 \mathrm{~mol} . \%$ spessartine. Zoning proximal to the internal garnet-quartz interface exhibits more variation along its boundary than the equivalent external interface: rim chemistry is space-dependent, and is variably truncated at c. 2 mol.\% spessartine, c. 2-10 mol.\% pyrope, c. 2-10 mol.\% grossular and 79-92 mol.\% almandine. Similarly, chemical zoning of individual porphyroblasts within a polycrystal is asymmetrical: steeper gradients are preserved towards the quartz-garnet interface than towards the garnetmica interface, and zoning is truncated towards the core (Fig. $4.7 \mathrm{a} \& \mathrm{~b}$ ).

Compared to Type A garnet, Type B populations comprise significantly smaller non-coalesced euhedral porpyroblasts. As with Type A, Type B garnet is spatially heterogeneous, and the distribution of crystal centres is clustered within quartzofeldspathic microlithons, near the margins of cleavage domains (Fig. 4.8). Compositional mapping reveals subtle chemical zoning (Fig. 4.6c). The core chemistry of the largest crystal (with a diameter of $560 \mu \mathrm{m}$ ) contains c. 4 mol.\% spessartine, 6 mol.\% pyrope, $8 \mathrm{~mol} . \%$ grossular and $82 \mathrm{~mol} . \%$ almandine (Fig. 4.7 f). Zoning is concentric, and rims of small garnets in the main cluster all contain c. 1 mol.\% spessartine, 6 mol.\% pyrope, 9 mol.\% grossular and 85 mol.\% almandine. Smaller crystals contain lower $\mathrm{Mn}$ in their cores, and there is an irregular, patchy distribution of $\mathrm{Ca}$ (Fig. 4.6c). Type B garnets are relatively devoid of inclusions. Some biotite associated with Type B garnet has been chloritised, and texturally late chlorite is present in the interstitial regions of clusters. 
The chemical composition of biotite in SIM3 is largely independent of its spatial disposition or proximity to other phases: coarse biotite at the margins of quartz-rich microlithons does not have a chemistry that is distinct from biotite disseminated along quartz grain boundaries or in cleavage domains, with constant K and Ti cpfu, 0.17 and $0.08-0.1$, respectively. However, there is a slight trend for systematically reduced $X_{\mathrm{Mg}}$ in biotite associated with Type B garnet (0.44-0.45), compared with Type A (0.46-0.47). The chemistry of white mica within domains containing Type A and B garnet does not vary systematically, though the chemistry is by no means homogeneous: a single cleavage domain contains muscovite with 24\%-27\% paragonite content. Plagioclase associated with Type B garnet has a narrow $X_{\mathrm{An}}$ range of $0.22-$ 0.23, and plagioclase adjacent to Type A garnet is zoned with pronounced albitic rims and anorthitic cores $\left(X_{\text {An }}\right.$ of 0.17 and 0.22 , respectively).

\section{MS7A}

Fine $<450 \mu \mathrm{m}$-long white mica in MS7A is muscovitic with $25 \%-27 \%$ paragonite and defines a well-developed $\mathrm{S}_{1}$ foliation, with narrow $2 \mathrm{~mm}$-wide quartz microlithons (Fig. 4.3 ). These horizons are commonly bound by fine biotite which is aligned at a slight angle to the foliation, and $0.3-2 \mathrm{~mm}$ foliation-parallel biotite is broadly concentrated in bands throughout matrix muscovite. Euhedral staurolite is scarce. Biotite in MS7A has $X_{\mathrm{Mg}}=0.45-0.49$ and $0.17-0.24 \mathrm{cpfu}$ Ti.

The matrix partially wraps euhedral garnet porphyroblasts, forming quartz strain shadows parallel to the foliation. Garnets are pre-to syn-kinematic with respect to final phase of matrix deformation and ilmenite, apatite and allanite inclusions are generally straight in the core and deflected to be continuous with the external $S_{1}$ at the rim. In some cases garnet has overgrown mica-rich strain caps, and garnet generally exhibits only minor evidence of resorption. Deformation is unlikely to have 
outlasted garnet growth. Garnet central sections exhibit chemical trends similar to previously described samples (Fig. 4.4c), with core-to rim decreases in spessartine and grossular contents from 16 to $1 \mathrm{~mol} . \%$ and c. 21 to $5 \mathrm{~mol} . \%$, respectively. Pyrope and almandine contents increase from core-to-rim from 5 to 14 mol.\% and c. 60 to 80 mol.\%, respectively. Grossular, pyrope and almandine contents all exhibit relatively homogeneous core zoning until c. $500 \mu \mathrm{m}$ from garnet rims.

XR- $\mu \mathrm{CT}$ imaging of the sample indicates that $0.08-1.67 \mathrm{~mm}$ garnet occupies 4.2 vol.\% of the rock volume. The average sphere-normalised garnet radius is $0.62 \mathrm{~mm}$, and the CSD is positively skewed with a skewness of 0.64 (Table 4.1 and Fig. $4.2 \mathrm{p}$ ). Unlike lower-grade samples, the garnet CSD does not exhibit a logarithmic decrease in crystal abundance with increasing crystal radius, but larger crystal frequencies decrease relatively linearly. The garnet crystal density is $\sim 15.8 \mathrm{~cm}^{-3}$, and some garnets are impinged.

\section{MAN22}

The structurally highest sample in the studied sequence contains abundant 200-600 $\mu \mathrm{m}$ kyanite and coarse isolated garnet porphyroblasts in a matrix comprising $0.2-1$ $\mathrm{mm}$ long biotite and white mica, and ilmenite and quartz (Fig. 4.3F). Matrix biotite has $X_{\mathrm{Mg}}=0.48-0.49$ and $0.20-0.23 \mathrm{cpfu} \mathrm{Ti}$ and white mica is muscovitic with $19 \%^{-}$ $22 \%$ paragonite. The pervasively deformed $\mathrm{S}_{1}$ schistosity is irregularly deformed and contains retrograde chlorite associated with garnet rims and biotite. All chlorite has $X_{\mathrm{Mg}}$ of $0.52-0.53$. Kyanite is aligned with the matrix foliation, and wraps garnet.

Garnets are anhedral and rounded, and contain abundant inclusions of quartz, ilmenite, biotite and apatite; plagioclase is only present in MAN22 as inclusions in garnet with $X_{\mathrm{Ca}}$ of 0.23 . Coarse biotite included in garnet has $X_{\mathrm{Mg}}=0.54$ and $0.21 \mathrm{cpfu}$ Ti. Straight-to-slightly curved internal foliations are generally aligned at a 
high-angle to the external foliation, and are often truncated by $1 \mathrm{~mm}$-wide inclusionpoor garnet rims. There is no foliation continuity between garnet and the matrix. The garnet population has sphere-normalised radii of 0.59-5.69 $\mathrm{mm}$, with an average radius of $2.5 \mathrm{~mm}$ (Table 4.1). It exhibits a lognormal CSD with a positive skew of 1.2 (Fig. 4.2). Garnets are evenly distributed throughout the scanned rock volume, and crystal density for this kyanite-grade sample is lowest of the sample set: $\sim 1.6$ garnets $\mathrm{cm}^{-3}$.

Core-to-rim major element zoning in the largest garnet porphyroblast from MAN22 is the least marked of all analysed samples (Fig. 4.4d). Pyrope and grossular contents do not vary significantly in core regions, with 11 mol.\% and 7 mol.\%, respectively, but reach outermost rim values of $16 \mathrm{~mol} . \%$ and $4-5 \mathrm{~mol} . \%$, respectively. The almandine distribution is similarly homogeneous in core regions, at $\sim 77$ mol.\%. However, there is an almandine hump beginning $c .1 .5 \mathrm{~mm}$ from the garnet rim, where almandine content reaches 81-82 mol.\%. Rimwards of this hump, almandine content decreases to $\sim 76$ mol.\%. Spessartine content broadly decreases from $4 \mathrm{~mol} \%$ in the core, to negligible in the rims. In the outermost $100 \mu \mathrm{m}$ there is an inflection in spessartine content, associated with a concomitant increase in $X_{\mathrm{Fe}}$.

\subsubsection{Whole-rock chemistry}

Table 4.2 displays the whole-rock major element chemistry for all key samples in this study. Bulk rock chemistries of these and additional samples are plotted in an AFM diagram in Fig. 4.9a, discriminated by Barrovian zone.

Samples generally contain less $\mathrm{MgO}$ than the average pelite of Symmes \& Ferry (1991), and occupy a range of $\mathrm{FeO}$ and A-values. There is no discernible correlation

between metamorphic grade and bulk composition, consistent with Dasgupta et al. (2009). The SIM samples (Fig. 4.2 d-f), which are the only rocks studied that contain 
coalesced Type A garnet, do not occupy a statistically distinct position in the AFM projection. However, there is a slight trend for these samples to have slightly elevated A-values. Samples that contain post-kinematic Type A garnet also contain, on average, more $\mathrm{Na}_{2} \mathrm{O}$ than samples with exclusively pre-to-syn kinematic porphyroblasts (Table 4.2). Mica phases are not notably more abundant in these samples (Table 4.3 .

\subsubsection{Summary}

In samples that exhibit a relatively homogeneous spatial distribution of porphyroblasts, garnet crystal size and modal abundance increase from the lower garnet to the kyanite zones, concurrent with a decrease in garnet crystal frequency (Fig. 4.2 \& Table 4.1). While resorption and retrogression of garnet has reduced the total garnet volume in some samples, it is not considered to be significant enough to have altered the systematic correlation between garnet crystal size and metamorphic grade. The $\mathrm{S}_{2}$ crenulation is only developed in the lowest grade samples, while higher grade samples exhibit a pervasively deformed $\mathrm{S}_{1}$ schistosity. If the assumption is made that deformation was coherent and continuous from the kyanite zone to the garnet zone, garnet is progressively pre, pre-to-syn, and syn-kinematic with respect to progressive phases of deformation. Matrix microstructures in each of these samples are relatively continuous at the hand sample scale. Chemical compositions of garnet rims and matrix phases are consistent with chemical equilibration of major elements at the hand sample scale.

The garnet to staurolite grade SIM samples are characterised by a strongly layered cleavage domain-microlithon microstructure. Porphyroblastic and matrix microstructures are spatially heterogeneous and at odds with textures in other samples: post-kinematic skeletal garnet populations are concentrated within quartz-rich do- 
mains and near the margins of strongly deformed ilmenite-rich micaceous domains. Garnet polycrystals comprise numerous porphyroblasts, each with a high-Mn core, concentric zoning with respect to $\mathrm{Mn}, \mathrm{Fe}$, and $\mathrm{Mg}$, and an absence of interstitial phases. Type A garnet typically overgrew and is spatially constrained by the extent of micaceous domains (Fig. 4.5b \& f), and porphyroblasts external to mapped clusters at microlithon-cleavage domain boundaries have chemical cores that are located on-or-near that boundary (e.g., dashed box, Fig. 4.63). Chemical gradients in Type A garnet indicate that garnet growth was faster towards micaceous domains than towards skeletal interfaces, which are generally associated with quartz. Smaller garnets in Fig. 4.6 are generally situated to the interior of clusters and contain lower core Mn. Quartz grain size is highly variable between microlithons.

Smaller uncoalesced Type B garnets are located predominantly within quartzofeldspathic microlithons, and contain lower core Mn (Figs. 4.8, 4.6a \& 4.7c). The chemistry of white mica does not differ between zones adjacent to Type A and B garnet, and biotite in the former contains slightly higher $X_{\mathrm{Mg}}$ than associated with adjacent Type B garnet. Plagioclase in SIM samples is significantly more abundant than in other samples (Table 4.3) and that associated with Type A garnet exhibits a broader compositional range and core-to-rim albite increases, whereas plagioclase associated with Type B is unzoned and less albitic (Fig. 4.9p).

Whereas there is no systematic variation in white mica chemistry with textural position in the samples studied, the paragonite content of white mica generally increases up to the staurolite grade, as expected due to progressive closure of the solvus with increasing $T$ (Fig. 4.9c). Other than the observed systematically heterogeneous chemistry of biotite in SIM samples, biotite composition is independent of textural association in any given sample. At the transect scale, biotite $X_{\mathrm{Mg}}$ increases up-grade, and $\mathrm{K}$ cpfu also varies systematically with grade (Fig. 4.9p). 
$\mathrm{Na}_{2} \mathrm{O}$ whole-rock concentrations vary systematically with the described changes in garnet texture: samples with post-kinematic coalesced Type A garnets and a heterogeneous matrix (i.e., SIM samples) contain higher $\mathrm{Na}_{2} \mathrm{O}$ wt.\% than the other samples studied (Table 4.2). There is a negative correlation between the core grossular content of the largest garnet in each sample and the respective bulk $\mathrm{Na}_{2} \mathrm{O}$ wt.\%, with an $\mathrm{R}^{2}$ correlation of -0.58 (Fig. $4.9 \mathrm{~d}$ ). The chemistry of MAN22 falls significantly off this trend.

\subsection{Crystallographic characterisation of coalesced garnet us- ing EBSD}

Euler orientation maps are used to investigate any potential non-random crystallographic organisation of garnet in two Type A polycrystalline clusters in SIM3 (Map 1 and Map 2, Figs. 4.10 and 4.11, respectively). Both map regions are located in a single thin section, and form a near-continuous structure in $3 \mathrm{D}$, that is approximately contained within and parallel to the structure of a single layer (Fig. 4.5 d).

\section{Map 1}

Map 1 mainly contains a garnet polycrystal which can be seen to have overgrown a sigmoidal cleavage domain, resulting in the incorporation of strongly structurealigned ilmenite inclusions the are continuous with the external foliation (Fig. 4.5d). The microstructure has a crystal extent that appears to have been limited by the location of the precursor interface between quartz and mica. EBSD shows that the crystallographic structure of this region comprises 56 garnet grains divided by highangle grain boundaries (Fig. 4.10 a). The central quartz-rich region contains numerous garnets $<100 \mu \mathrm{m}$ in diameter. Subgrains are scarce and large when present, but Euler orientation maps do show internal grain misorientations that are not defined 
by MTEX as subgrain boundaries (red dashed boxes, Fig. 4.10a). Mean angular deviation maps (not shown) indicate that these misorientations $\left(<8^{\circ}\right)$ occur over a wider region than the case for a discrete grain or subgrain boundary. In addition to these broad misorientation boundaries, large individual garnets in Map 1 also exhibit continuous internal lattice misorientations up to $\sim 5^{\circ}$ (Fig. 4.10 ).

Misorientations between adjacent garnet porphyroblasts have a crystallographicallyimposed upper limit of $62.8^{\circ}$ (Mackenzie, 1958). Misorientation angles across neighbourpair garnet-garnet boundaries in Map 1 may deviate from a random distribution: misorientations of $\sim 30^{\circ}$ and $\sim 47^{\circ}$ are most abundant (Fig. 4.10 b). Random-pair misorientations more commonly have low degrees of misorientation. The neighbour-pair misorientation axis distribution (not shown) is also non-random: there is strong clustering of axis around a [001] of garnet in Map 1. However, given the limited number of grains in this microstructure, their non-random distribution may not be statistically significant. Considering that the deviation of the untextured angle distribution calculated with the SIM3 orientation distribution function (ODF; Fig. 4.10b, blue line) varies significantly from the random/untextured angle distribution (Fig. 4.10p, red line), lack of statistical significance may be important. Energetically favourable and specific orientation grain boundaries (assessed by the characterisation of coincident site lattice (CSL) boundaries) are rare. Of all high angle grain boundaries in Map 1, only $\sim 3 \%$ are CSL boundaries with the majority $\Sigma>9$, where a lower $\Sigma$ represents a closer fitting boundary (e.g., twin boundaries are $\Sigma 3$ ) to a maximum of $\Sigma 49$ (Brandon, 1966).

Figure 4.10; and d shows garnet pole figures and their corresponding ODFs accounting for differences in grain sizes, where grain size is defined by the number of pixels, with edge-touching garnets removed. Despite the changing orientation of the matrix over which garnet grew, pole figures and ODFs of the largest garnets in Map 
1 show a strong crystallographically preferred orientation (CPO) with $\{100\}$ pole clustering of c. 8.4 multiples of uniform density (MUD) (Fig. 4.10d). There is some dispersal of $\{100\}$, but outside the maxima the density is generally $<0.4$ MUD. All

poles are consistent with having been rotated around a $\{111\}$ pole at $317 / 09$ with 8.5 MUD by approximately $15^{\circ}$. The poles for small garnets $(<1000$ pixels $)$ have a weak $\mathrm{CPO}(\mathrm{MUD}=3.5)$ that aligns with the $\mathrm{CPO}$ in larger grains, but have not rotated around the $\{111\}$ pole (Fig. 4.10 $)$.

\section{Map 2}

The polycrystalline cluster in Map 2 comprises 211 garnet porphyroblasts with distinct lattice orientations, divided predominantly by high-angle grain boundaries (Fig. 4.11a). Unlike for Map 1, the grain boundary angle distribution of both garnet-garnet neighbour-pairs and random-pairs is comparable to the calculated uncorrolated misorientation angle distribution (Fig. 4.11b) and the neighbour-pair misorientation axis distribution (not shown) is similarly uncorrelated. Of all high angle grain boundaries in Map 2, $\sim 10 \%$ are CSL boundaries with the majority $\Sigma>9$. The low abundance of CSL boundaries observed in both EBSD maps from SIM3 is consistent with them having formed via a random origin, opposed to a low energy configuration which may result from systematic grain rotation (Whitney \& Seaton, 2010).

Crystallographic orientations do not vary significantly within a given garnet grain, and there is little internal substructure. Grain and subgrain boundaries correlate with and do not truncate patterns of major element chemical zoning (Figs. 4.6 a \& 4.11), and larger marginal garnets with high Mn-cores exhibit garnet-garnet grain boundaries broadly perpendicular to the orientation of the external garnet-matrix grain boundary, and the orientation of the matrix foliation. Subgrain boundaries are scarce.

As calculated for Map 1, Figs. 4.11k and d show garnet pole figures and their 
corresponding ODFs accounting for differences in grain sizes. Small garnets $(<1900$ pixels) are only present in the center of the polycrystal (and generally decrease in size with increasing distance from cleavage domains) but make up $89 \%$ of all indexed garnet grains, and have highly irregular grain boundaries with quartz. They have a randomly distributed ODF (Fig. 4.11d) and a negligible CPO. Large garnet grains ( $>1900$ pixels) are present towards the margins of the polycrystal, and they exhibit a weak $\mathrm{CPO}$ in the ODF (Fig. 4.11,) with maximum MUD in $\{001\}$ of 3.6, and a discernible orientation preference in $\{110\}$ and $\{111\}$. Notably, large crystals along the linearly structured rightmost margin of Map 2 (grey dashed box, Fig. 4.12a) are oriented with $\{100\}$ approximately parallel to the trace of the ilmenite foliation included in garnet and the adjacent matrix foliation (cubes in Fig. 4.12 a, pole figures not shown). When only these grains are considered, the ODF defines a pronounced $\mathrm{CPO}$ with an average MUD $=7.4$.

\subsubsection{Epitaxial relationships}

A low index plane of metamorphic garnet (i.e., $\{100\},\{011\}$ or $\{111\}$ ) has been shown to commonly exhibit parallelism with a (001) basal mica plane where epitaxial relationships between the two have been identified (Powell, 1966; Spiess et al. 2007; Robyr et al., 2009; Cruz, 2011; Moore \& Carlson, 2015). Most often, the $(001)_{\text {mica }} / /\{110\}_{\text {grt }}$ relationship is present.

To assess whether the CPOs in SIM3 might be related to favourable orientation development by epitaxial crystallisation, MTEX was used to simulate pole figures for all possible symmetric variants of mica that may have resulted in the observed garnet orientation, assuming the $(001)_{\text {mica }} / /\{110\}_{\text {grt }}$ relationship holds. Given the lack of inclusions of potentially precursor mica in garnet, and unindexable diffraction patterns for matrix mica, the orientation of precursor matrix phases has been indirectly 
inferred. Similar to the approach presented by Robyr et al. (2009), the coherent alignment of the long axis of ilmenite in both the matrix foliation and as inclusions in garnet may permit the use of these inclusions as a proxy for the orientation of precursor micas upon which garnet may have formed in certain regions. Visual inspection of the trace of the long axis of the ilmenite inclusions in thin section, coupled with plane fitting using $\mathrm{XR}-\mu \mathrm{CT}$ data gives a reasonable constraint on the precursor mica orientation at the geochemical core of each grain in the garnet polycrystal in Map 2. Constraints on the orientations of precursor micas have been obtained in Map 2 (boxed region, Fig. 4.12); this region was selected as the orientation of the precursor foliation is systematic and thus possible to constrain with some degree of confidence. In other regions of Map 2, and in Map 1, the included ilmenite orientation is either highly variable or is absent, and so the precursor orientation cannot be reliably estimated. Error is likely to be introduced at this stage; fit is by visual inspection and is thus susceptible to bias. In most cases, the estimate of the trace of the foliation is of higher quality than that of the relative dip, out of the plane of the section. Inclusions are also not evenly oriented in the crystal, and therefore an average has been taken. Wherever possible, the value chosen as the orientation of the trace of the ilmenite corresponds best to the ilmenite closest to the chemical core of a given crystal (Fig. 4.6a). The best estimate is that the magnitude of this error might be as large as $15^{\circ}-20^{\circ}$, but could be significantly smaller.

Moreover, the ilmenite crystal structure is similar to that of phyllosilicates in that the c-axis (the pole to $\{0001\}$ ) is parallel to the short-axis of the mineral. Thus, the orientation of the $\{0001\}$ ilmenite pole may be used to infer the orientation of the basal plane of mica. A small proportion of the included ilmenite has been indexed with EBSD, and is used in addition to the plane fitting method described above. This method assumes that there is a perfect correspondence between the orientations 
of mica and ilmenite and that the orientation of mica is broadly coherent. Given the high aspect ratio of the ilmenite habit, ilmenite may rotate around its long axis, introducing additional uncertainty. Because this uncertainty is so difficult to constrain and because ilmenite is so poorly indexed, microstructural constraints on ilmenite are used as secondary confirmation of the foliation orientation.

In Fig. 4.12, the estimated orientation of the measured foliation in each given grain (numbered 1-8) is shown in red. Where ilmenite is coarse enough to have been indexed effectively in the same grain, the orientation of the c-axis and the location of the basal plane is plotted in a corresponding $\{0001\}$ pole figure (in blue). Given the known orientation of each garnet crystal, the green dots represent both this orientation (in $\{001\}$ ) and also all the possible calculated symmetric variants of the $\{001\}$ plane in mica that maintains $(001)_{\text {mica }} / /\{110\}_{\text {grt }}$. Reasonable coincidence between a reasonably oriented (001) pole (given petrographic knowledge) and an estimate of the orientation of the precursor mica foliation may support the idea that garnet inherited the orientation of the precursor. Results of this analysis vary considerably, and are summarised in Table 4.9. Crystals [1], [2], [4], [5], [7] and [8] have an angle between the estimated inclusion orientation and the best-fit garnet/mica pole that is $<20^{\circ}$, a relatively good correspondence given the high degree of uncertainty inherent to the method used. Garnets [1] and [5] have similar angular discrepancies between garnet and both foliation measurements. For garnet [8], there is almost perfect correspondence. For most grains, the fit between the $(001)_{\text {mica }} / /\{110\}_{\text {grt }}$ pole and the measured ilmenite orientation is less pronounced, with a range of $18.8^{\circ}-43.8^{\circ}$ between the two.

Mica has not been observed in this study as an inclusion at the site of inferred garnet nucleation. It is hence impossible to unequivocally provide evidence for an epitaxial relationship. However, given the limited data obtained here, it seems possi- 
ble that an epitaxial relationship holds in some cases, such that a (001) of precursor mica parallels a $\{110\}$ in garnet. Microstructural observations are consistent with this result, given the documented $\mathrm{CPO}$ of garnet at cleavage domain-microlithon boundaries in Map 2 (that strengthens in a region with a near-single ilmenite orientation), and the apparent preference for post-kinematic garnet to replace strongly foliated micaceous domains.

\subsection{Metamorphic conditions of garnet crystallisation}

In order to test whether the microstructural differences described above may be attributable to differences in the timing of onset of garnet crystallisation, the relative orientation of the prograde path, and/or length of the path, metamorphic conditions of garnet crystallisation have been estimated. Isopleth thermobarometry and THERIA_G modelling has been applied to estimate the $P-T$ trajectories associated with garnet crystallisation in MAN1 and MS7A, given their homogeneous distributions of major phases at the hand sample scale and the idioblastic habit of garnet in these samples. This approach has already been successfully used by Gaidies et al. (2015) and George \& Gaidies (2017), in which the $P-T$ evolution of 24-99 has been wellconstrained. MAN22 could not be modelled with THERIA_G given the poor isopleth intersection, but phase equilibra modelling provides broad constraints on peak metamorphic conditions. The Ti-content of biotite (Henry et al. 2005) was used as an additional test of peak metamorphic conditions.

On account of the heterogeneous distribution of phases in SIM3, the location dependent variations in mineral compositions, and the highly irregular crystal shapes of garnet, a different approach was applied to this sample. In addition to using the whole-rock chemical composition as determined by XRF, the XR $\mu$-CT dataset was 
used in conjunction with seven thin sections to estimate modal proportions in rock fractions representative of the rock volumes containing Type A and Type B garnets. These were combined with representative phase analyses from each fraction to estimate two representative bulk compositions across smaller equilibration volumes. Core isopleth intersections were determined, after which chemical fractionation associated with garnet growth along a stepwise $P-T$ path fitting the observed chemistries was simulated for metamorphism of both rock fractions. For Type A garnet, the core of the largest garnet in the mapped cluster contains the highest spessartine content of the respective rock fraction (Fig. 4.7a). This chemistry is used as the earliestformed garnet, and the crystal rims in contact with cleavage domains are interpreted as the youngest garnet portions, formed at peak metamorphic conditions. For Type B garnet a similar approach was used, simplified by the relatively concentric zoning.

\subsubsection{Crystallisation of isolated porphyroblastic garnet}

Figure 4.13 shows the combined results of core isopleth thermobarometery for each of the key studied samples with non-coalesced garnet morphologies (excluding 24-99, which is described in George \& Gaidies, 2017). The topology of all phase equilibria diagrams is very similar, with low-variance fields bounding the stability field of staurolite-bearing assemblages at $c .550-650{ }^{\circ} \mathrm{C}$. The low- $P$ limit of plagioclase stability varies significantly, as does the location of the garnet-in curve, a consequence of its sensitivity to bulk $\mathrm{MnO}$ content.

MAN1 garnet core isopleths (Fig. 4.13 a) intersect relatively tightly at $500{ }^{\circ} \mathrm{C}$ and 3.8 kbar, approximately $5{ }^{\circ} \mathrm{C}$ above the garnet-in curve. The isopleth intersection for garnet in MS7A is less well-constrained, at $510-520{ }^{\circ} \mathrm{C}$ and $4.6-5.2 \mathrm{kbar}$; this is c. $10-20{ }^{\circ} \mathrm{C}$ above the garnet-in curve (Fig. 4.13b). Excluding constraints using the broadly spaced pyrope isopleths, MS7A core chemistry intersects much more tightly 
at $510{ }^{\circ} \mathrm{C}$ and $5 \mathrm{kbar}$. Core isopleths of MAN22 do not intersect well in any given divariant field (Fig. 4.13c): almandine and spessartine isopleths intersect at c. 540 ${ }^{\circ} \mathrm{C}$ and $4.2 \mathrm{kbar}$ in an assemblage containing plagioclase, garnet, chlorite, muscovite, biotite, ilmenite and quartz, but do not coincide with grossular and pyrope, which only intersect in a field without chlorite and containing staurolite. While every effort was made to ensure a central section was obtained, slight differences between the observed compositions and equilibrium garnet-in curves may be related to a lack of coincidence between the geometric and compositional core of garnet. Alternatively, non-equilibrium compositions of garnet may be present after significant overstep of the garnet-forming reaction (Gaidies et al., 2011; Castro \& Spear, 2016). In MAN22, the abundance of inclusions in garnet possibly promoted diffusional homogenisation, despite its large radius.

Results of garnet crystallisation modelling using THERIA_G are presented in Fig. 4.14. In general, the observed growth zoning in garnet from MAN1 and MS7A can be predicted well by crystallisation along the $P-T$ paths presented in Fig. $4.14 \mathrm{a}$ and b, respectively, using CSDs determined using XR- $\mu \mathrm{CT}$ (Fig. 4.2), and a heating rate of $100{ }^{\circ} \mathrm{C} \mathrm{Myr}^{-1}$, in line with previous findings of George \& Gaidies (2017).

The best-fit $P-T$ path for MAN1 (Fig. 4.14 c) predicts incipient garnet crystallisation in an assemblage with muscovite, biotite, chlorite, plagioclase, quartz and ilmenite at $495^{\circ} \mathrm{C}$ and 3.6 kbar. Crystallisation proceeds in the same assemblage along a relatively linear $P-T$ path to $555^{\circ} \mathrm{C}$ and c. $5.1 \mathrm{kbar}$, with a discrete and approximately isobaric fluctuation in the $T$ evolution starting at $514{ }^{\circ} \mathrm{C}$ and ending at $524^{\circ} \mathrm{C}$. This fluctuation is included as it is required to match the positioning of a symmetrical discontinuity in the rim-rim profile of MAN1 (Fig. 4.4 ). While these $T$ oscillations may be exaggerated in the model, it highlights the presence of a discontinuity along the $P-T$ path. Interestingly, its location approximately matches 
that of the isobaric period in models of Gaidies et al. (2015). Peak metamorphic conditions represented by garnet rims are consistent and within error of peak- $T$ estimates using Ti-in-Bt thermometry. Along this $P-T$ path (Fig. 4.14 a) the pyrope content is reproduced well from core-to-rim, as is the general distribution of grossular. The slight elevation of the core isopleth intersection above the garnet-in curve means that the model overpredicts core spessartine content by $1 \%-2 \%$. Outwards from the core, spessartine and almandine decrease and increase, respectively, more than in the observed compositional profiles. This may be related to the input CSD used in the simulation: if there were fewer large garnets relative to small ones, less volume would be distributed on early formed crystals and the period of comparatively rapid core growth would continue to higher $P-T$ s. Furthermore, the size of the largest garnet in MAN1 is underpredicted by the models by $c .50 \mu \mathrm{m}$. This can be explained by the large volume fraction that quartz inclusions take up in the garnet core (Fig. 4.4 ). Whole-rock XRF chemistries do not 'see' inclusions in garnet. Thus, for an inclusion-rich population, porphyroblast volume (and hence, radius) will exceed that predicted by the inherently inclusion-free model. Inclusions in MAN1 take up 12.1\% of the total volume of the measured porphyroblast. If the assumption is made that all porphyroblasts in the population contain the same inclusion density and if that inclusion volume is added to the model, the predicted radius is in line with the measured radius.

The modelled zoning of the largest garnet in staurolite-bearing MS7A is presented in Fig. 4.14b. Core-rim grossular and pyrope contents are well replicated by a model that crosses the garnet-in at $479{ }^{\circ} \mathrm{C}$ and $4.7 \mathrm{kbar}$ and proceeds to c. $510{ }^{\circ} \mathrm{C}$ and 5.1 kbar, with subsequent shallowing of the gradient of the path up to $570{ }^{\circ} \mathrm{C}$ and 6.3 kbar (Fig. 4.14b). With this best-fit model the chemical composition of early-formed garnet is overpredicted with respect to spessartine (c. 5 mol.\% higher than observed) 
and underpredicted by the equivalent amount of almandine.

Garnet growth in MS7A is predicted to have ended with the approximately simultaneous loss of plagioclase from the assemblage at $574{ }^{\circ} \mathrm{C}$ and $6.4 \mathrm{kbar}$. The prograde path must have then shallowed in gradient and proceeded to $\sim 581{ }^{\circ} \mathrm{C}$ to stabilize staurolite-bearing assemblages and to $583^{\circ} \mathrm{C}$ to destabilize prograde chlorite-bearing assemblages. While we have been unable to fit the outermost $100 \mu \mathrm{m}$ garnet chemistry, it may be the case that it was the result of low-Ca garnet growth at a $T$ above the staurolite-out, with metastable persistence of staurolite in the rock. Peak conditions estimated using this model are supported by the $580{ }^{\circ} \mathrm{C}$ obtained using Ti-in-Bt thermometry (Fig. 4.13b).

Although it has not been possible to forward model garnet growth in MAN22, the observed peak assemblage is predicted to have formed between c. $\quad 650-700{ }^{\circ} \mathrm{C}$ and 6.5-8 kbar (Fig. 4.13c). However, Ti-in-Bt thermometry predicts peak-Ts of $605 \pm$ $23{ }^{\circ} \mathrm{C}$, a difference that may relate to the fact that the Henry et al. (2005) calibration has been shown to be most reliable for Ps of 4-6 kbar.

\subsubsection{Crystallisation of irregular garnet in SIM sample}

The topologies of phase equilibria models associated with Type A and Type B garnet in SIM3 (Fig. 4.15a and b, respectively) are comparable to those presented for other samples (Fig. 4.13). Almandine, spessartine and grossular isopleths representing the earliest core chemistry in Type A garnet intersect at $530{ }^{\circ} \mathrm{C}$ and $4.4 \mathrm{kbar}$ in a divariant field containing garnet, muscovite, quartz, chlorite, biotite, plagioclase and ilmenite, with the less well-constrained pyrope isopleth $c .10{ }^{\circ} \mathrm{C}$ higher (Fig. 4.15a). This intersection is c. $15^{\circ} \mathrm{C}$ above the garnet-in for this bulk chemistry, a difference that may be related to the difficulty in transecting the compositional core of the irregular garnet morphology. Stepwise forward modelling accounting for fractionation of garnet 
along a $P-T$ path from this core isopleth intersection to $577{ }^{\circ} \mathrm{C}$ and $6.1 \mathrm{kbar}$ best predicts the outermost rim composition (Fig. 4.15;).

The isopleth intersection of Type B garnet cores using the bulk-rock composition of the corresponding rock fraction is at c. $550{ }^{\circ} \mathrm{C}$ and $5 \mathrm{kbar}$ (Fig. $4.15 \mathrm{~b}$ ). The composition of Type B garnet is modelled from these conditions to $580{ }^{\circ} \mathrm{C}$ and $6.1 \mathrm{kbar}$ using a two-step path (Fig. 4.15d), given the small grain size and low modal abundance of garnet in this population. Only the observed almandine and grossular content can be simultaneously predicted, but the divariant field of the observed assemblage has a comparable stability range to that in the Type A system.

The similarities between the predicted peak $P-T$ conditions and the overlapping $P-T$ paths of the Type A and Type B systems indicate that Type B garnet may have crystallsed as a distinct population, subsequent to the earlier nucleation and growth of Type A garnet populations. In both model systems, predicted $T$ s of rim growth are comparable to that obtained with Ti-in-Bt thermometry, at $567 \pm 23{ }^{\circ} \mathrm{C}$. In concordance with these results, the topology and $P-T$ paths calculated using the bulk rock composition of the entire sample is very similar, although the garnet-in curve is predicted to be higher and lower than compared to that for Type A and B bulks, respectively, at c. $525^{\circ} \mathrm{C}$.

\subsection{Implications}

\subsubsection{Polycrystal development in SIM3}

\section{Progressive nucleation and coalescence}

There is no evidence for post-crystallisation coalescence and dissolution-precipitation creep (e.g., Wassmann \& Stöckhert, 2013), strain-induced lattice recrystallisation or reorientation (e.g., Prior et al., 2002; Spiess et al., 2001), or trapped phases between 
grain boundaries in Map 2, so the shape and disposition of the garnet structures is interpreted to be primary. Compositional maps of different parts of SIM3, coupled with EBSD data, provide strong evidence for spatially-dependent progressive nucleation and coalescence of garnet porphyroblasts to form polycrystals. In the case that the system containing the polycrystal was approximately equilibrated with respect to $\mathrm{Mn}$ in these small local chemical systems, the spessartine content of garnet can be used as a relative timeline for its nucleation (e.g., Daniel \& Spear, 1998,): Fig. 4.67 indicates that marginal garnets in the polycrystal formed earlier, and central garnets formed later.

A weak CPO is documented at the scale of the Map 2 cluster, enhanced when linear tracts of grains are assessed. Spiess et al. (2001) interpreted a similar CPO that first developed in the exterior garnets of a polycrystalline garnet cluster to be the result of differential growth rates and rotation of grains upon their impingement, with rotation of lattices towards some coincident orientation, driven by the reduction of subdomain boundary energy associated with misorientation loss (Herrmann et al., 1976, Dobbs et al., 2003). However the absence of non-random low-energy CSL boundaries indicates that independently nucleating and coalescing garnets did not rotate together into special low-energy orientations, as is the case for some coalesced porphyroblasts presented in Whitney et al. (2008). Moreover, unlike in Prior et al. (2002), there is no systematic and/or statistically significant orientation change across grain boundaries separating adjacent garnets in either map (Figs. 4.11b and 4.10p), and there is no preference for low degrees of misorientation across neighbour-pair boundaries. If crystallographic orientations of garnet had interacted upon impingement, then neighbour-pair misorientations should favour low misorientations to a greater extent than random-pair (Wheeler et al., 2001). It is therefore unlikely that late crystal impingement caused lattice rotation into the documented CPO. 
It seems instead that garnet grains which make up the polycrystal in Map 2 grew independently, and the CPO of external grains in the cluster instead partially developed as a result of epitaxial crystallisation of potentially early-nucleated garnet on mica at or near the boundaries between quartzose microlithons and micaceous cleavage domains. This is consistent with the non-central location of chemical cores in a given grain, but the trend is not immediately evident at the cluster scale as a consequence of the variable orientation of the included (and precursor) foliation upon which garnet nucleated. Because of difficulties constraining the precursor orientations, internal garnets or those with a poorly-defined inclusion orientation have not been tested for epitaxial relationships. However, the absence of aligned inclusions in latenucleated internal porphyroblasts in the cluster in Map 2 indicates that they may not have replaced mica phases, consistent with the preference for smaller internal garnets to have dispersed orientations with limited CPO.

Given the more extensive and most evolved chemical zoning present towards rims in contact with micaceous domains, growth is interpreted to have continued outwards towards mica-rich domains following impingement (Fig. 4.6a). The steeper compositional gradients and smaller porphyroblast sizes towards the interior of the cluster in Map 2 (Fig. 4.7) suggest that growth rates were slower here than towards the exterior margin of the polycrystal given their contact with quartz \pm plagioclase and the relative decrease in abundance of garnet-forming components (although the relative increase in the number of distinct crystals perhaps suggests an increase in the number of favourable nucleation sites). The proximity of external regions in Map 2 to micaceous domains may have facilitated continued growth, given the relative abundance of reactive components. More rapid coalescence of large, early-nucleated grains along and near boundaries may have also further enhanced differential growth rates by acting as an additional barrier for diffusion of components to grains in the centre, 
ultimately resulting in the early cessation of growth in the interior and the truncation of core-rim chemical zoning patterns. The relative absence of $\mathrm{Ca}$ in mica-rich domains compared to quartzo-feldspathic domains is consistent with the observation of decreases in grossular content to the marginal rims of polycrystals and limited transfer of Ca to the interior of the cluster (e.g., Spear \& Daniel, 2001).

\section{Internal strain accommodation}

Unlike the record of abundant nucleation and impingement in Map 2, Map 1 contains microstructures that evince a different crystallisation history-one with more limited nucleation (given the relative scarcity of individual grains), but more significant growth parallel to a preexisting structure. Most notably, these larger grains contain up to $5^{\circ}$ of lattice misorientation, but cluster around a single orientation. While Map 1 has not been chemically analysed and the location of chemical cores are unknown, we consider it likely that chemical zoning of each discrete porphyroblast is parallel to grain boundaries, as is the case in Fig. 4.6a and b.

Internal misorientations within garnet are commonly associated with lattice reconfigurations as a consequence of plastic post-crystallisation deformation. They have been documented in lower-crust to upper-mantle high- $T$ rocks. Garnets in these samples contain numerous subgrains which formed as a result of dislocation creep and recovery, dynamic recrystallisation and diffusion creep assisted grain boundary sliding (Prior et al. 2000; Prior et al., 2002, Storey \& Prior, 2005). At Ts $<450{ }^{\circ} \mathrm{C}$, the presence of large internal garnet misorientations has been attributed to lattice accommodation of deformation associated with high fault-associated strain rates of $10^{-14}-10^{-16} \mathrm{~s}^{-1}$ (Trepmann \& Stöckhert, 2003; Mukhopadhyay et al., 2017). There is no evidence for such strain rates in SIM samples, and these rocks did not reach $T$ s expected for the decline in garnet strength and documented onset of garnet creep 
in eclogites $\left(600-800{ }^{\circ} \mathrm{C}\right.$, Voegelé et al., 1998; Wang \& Ji, 1999; $700{ }^{\circ} \mathrm{C}$, Storey \& Prior, 2005). Moreover, as for Map 2, if the garnet structure in Map 1 was derived from post-crystallisation deformation, smaller subgrains would be expected to develop adjacent to high angle grain boundaries (Wassmann \& Stöckhert, 2013), microstructural continuity between inclusions in garnet and the matrix would not be maintained as effectively, and it is unlikely that crystal orientations within the folded structure would coincide strongly about a single orientation (Fig. 4.10 d).

We therefore interpret the continuous misorientation across grains comprising the polycrystal in Map 1 as a primary feature. It may be the result of limited nucleation and extensive post-kinematic growth parallel to (001) of mica: as garnet overgrew the strongly deformed precursor matrix (spatially limited by the relative availability of components in cleavage domains compared to surrounding quartz) the relatively large unit cell of garnet may have accommodated the changing orientation of mica with slight lattice distortions. Conversely, the relative abundance of distinct crystals comprising the polycrystal in Map 2 compared to Map 1 may have negated the need for the development of internal misorientations to accommodate the changing matrix orientation. The reasons for these different tendencies for nucleation versus growth in Map 1 and 2 are unclear, particularly given their adjacent locations and similarities with respect to the abundance of mica phases and similar quartz-mica boundaries in both zones. Moreover, given evidence for epitaxial crystallisation of garnet on mica phases in this and other studies (e.g., Spiess et al., 2007; Robyr et al., 2009; Cruz, 2011), and the apparent growth control by precursor micas in the structure in Map 1, the systematic CPO and changing ilmenite orientation is contradictory. Reasons for this are unclear and are not investigated here, but may relate to growth of garnet in a significant strain field. 


\subsubsection{Transect continuity across the IMS}

Figure 4.16a summarises the main thermobarometric constraints of this study, George \& Gaidies (2017), and of relevant samples from Gaidies et al. (2015). While models of garnet growth have not been obtained for MAN22, the peak $P-T$ conditions predicted for the observed divariant assemblage provide a broad constraint on metamorphism in the kyanite zone. Partial $P-T$ paths are nested and have similar gradients, with progressive increase of peak metamorphic conditions towards the MCT. This mirrors the systematic increase in Lu-Hf garnet core ages towards the MCT determined by Anczkiewicz et al. (2014): $13.7 \pm 0.3 \mathrm{Ma}$ in the kyanite zone, $12.8 \pm 0.3 \mathrm{Ma}$ in the staurolite zone, and $10.6 \pm 0.2 \mathrm{Ma}$ in the garnet zone. A kyanite zone garnet rim was constrained to $9.9 \pm 3.8 \mathrm{Ma}$, and peak metamorphic conditions were interpreted to have been achieved approximately simultaneously throughout the IMS after $c .6$ Myr of Barrovian metamorphism. Peak conditions in the garnet zone were estimated to have occurred at a similar time of 10-9 Ma by Mottram et al. (2014b). Following constraints of Faak et al. (2012) and Gaidies et al. (2015), maximum metamorphic $T$ s are considered likely to coincide closely with maximum $P$ s, with steep retrograde paths soon after peak metamorphism.

Phase equilibria and forward modelling of garnet growth in SIM3 confirm that it was metamorphosed along approximately the same prograde trajectory experienced by other rocks in the transect, although peak Ps exceed that determined for the staurolite grade SIM5 and MS6A in Gaidies et al. (2015). Results of a $P-T$ path trajectory calculated using the single bulk derived from whole-rock XRF of SIM3 do not differ significantly from constraints obtained using bulks representative of more restricted chemical equilibration (Fig. 4.15). So, differences in the microstructures of Type A and Type B garnets are interpreted to reflect differences in the timing of crystallisation as controlled by local compositional variations across $<3 \mathrm{~cm}$ within a 
chemically layered system. Smaller Type B populations reflect an early stage of the growth history of Type A garnet, and with continued growth, might be expected to form similar coalesced morphologies.

As proposed by Dasgupta et al. (2004) and Gaidies et al. (2015), the similarity and continuity between the modelled $P-T$ evolution of SIM3 and those obtained from samples structurally above and below SIM3 potentially attests to metamorphism of the exposed sequence (and the Daling Group as a whole) as a single coherent and continuous unit with progressively shallowing structural levels at increasing grade, as opposed to numerous and widespread tectonic slivers (e.g., Bollinger et al., 2006, Catlos et al., 2004). However, post-kinematic microstructures in SIM3 are anomalous to those expected for a fully continuous IMS that experienced a coherent $P-T$ evolution and continuous deformation, and while their development in the local rock system can be explored, the reason for their presence in the sequence is unclear. Figure 4.16 b summarises key $T-t-D$ constraints determined from this and other studies. The MCT-parallel and northerly-dipping $\mathrm{S}_{1}$ is pervasive and often deformed, and at the lowest grades (MAN1, 24-99) merges into a discrete crenulation cleavage, $\mathrm{S}_{2}$. Geochronological constraints on progressively earlier garnet growth in higher grade samples compared to lower grade samples (Anczkiewicz et al., 2014) coupled with a systematic change in the extent of inclusion-matrix continuity from low-to-high grade in all but the SIM samples is consistent with approximately continuous deformation across the sequence and the sequential attainment of thermal conditions suitable for garnet growth. In order to account for the post-kinematic nature of garnet growth in the SIM section, it may have been spatially decoupled from the otherwise coherent transect during its metamorphism, such that the timing of garnet growth and/or the deformation history in the region contrasts with that in rocks around it. Alternatively, in the case that the SIM sequence is structurally continuous with adjacent 
units, a change in the accommodation of deformation in the sequence containing SIM samples may have facilitated heterogeneous crystallisation of garnet, with the microstructures only apparently later than those in adjacent regions. Delay in garnet crystallisation as a consequence of sluggish reaction kinetics may have contributed to the delay in relative timing of growth (e.g., Waters \& Lovegrove, 2002, Pattison \& Tinkham, 2009), though would necessitate exceedingly high degrees of overstep of the predicted conditions at which garnet became stable in order to commence growth after the end of deformation.

Without geochronological constraints, the absolute timing of post-kinematic garnet growth in SIM3 cannot be discerned. However, we consider it most likely that the unit is decoupled in some way. To account for potential thermal decoupling and a resultant difference in the relative timing of garnet growth, tectonic discontinuities might be expected to divide the SIM sequence from adjacent exposures (e.g., Mottram et al, 2014a). Interestingly, the location of the SIM samples approximately coincides with the position of the Mangan-Sankhalan Thrust of Chakraborty et al. (2017), the MCT1 defined by Catlos et al. (2004) and the MCT2 defined by Bhattacharyya \& Mitra (2009). Its location as determined by Bhattacharyya \& Mitra (2009) is based on the transition from greenschist to amphibolite grade lithologies between their Daling Group and Lingtse Gneiss. However, other than the discordant SIM zone presented here, there are no major lithological, geochemical (Mukhopadhyay et al., 2017), or geochronological (Anczkiewicz et al., 2014) discontinuities associated with this transition above and below their MCT2, and there is an absence of evidence for widespread duplexing in the LHS footwall between this SIM region and the northern MCT. As initially suggested by Chakraborty et al. (2017) to explain the exposure of the Lingtse gneiss parallel to the MCT, the post-metamorphic development of minor fault splays diverging from the roof thrust of the duplex beneath the Daling Group could account 
for the occurrence of a discrete lithological package within an otherwise structurally coherent sequence.

We therefore suggest that samples from the SIM sequence represent an out-ofsequence section of the Daling Group which was emplaced along minor fault structures (potentially the proposed Mangan-Sankhalan Thrust) within an otherwise continuous IMS. Metamorphism of the unit as a coherent sequence (e.g., Dasgupta et al., 2009) and later tectonic inversion as a single block and subsequent thrust-accommodated tectonics (e.g., Chakraborty et al., 2017) accounts for the relative similarities in $P-T$ paths. Given their very similar metamorphic histories, slight differences in structural levels between the SIM samples and those directly adjacent to it cannot be resolved. This decoupling of the section, potentially both thermally and kinematically, does not specifically constrain the timing of garnet growth compared to adjacent outcrops in the IMS. However, if derived from a different level of the Daling Group, it is conceivable that both the nature of the protolith and the deformation history was abruptly different from the continuous variation across the rest of the sequence. This is supported by the difference in bulk chemistry of the SIM samples.

\subsubsection{Grade-related texture variations}

While the tectonometamorphic histories do not directly elucidate texture development, the predicted $P-T$ paths may shed some light on the systematic changes in size and abundance of garnet with change of grade in most samples of the IMS. The

volumes of garnet observed in the non-coalesced samples (Table 4.1) are reproduced to within $0.3-1.5$ vol.\% along the best-estimate $P-T$ paths in Fig. 4.16 a (also combining results from 24-99 from George \& Gaidies, 2017). Patterns of volume increase with T from the garnet to kyanite zone are generally in line with the relative changes in garnet volume predicted for the average metapelite of Symmes \& Ferry (1991), despite 
variation in the relative positioning of reactions, the volume percent, and the presence of melt before the stability of kyanite-bearing assemblages (Fig. 4.166). As expected, garnet volume increases to the point at which staurolite-bearing assemblages become stable, after which there is no garnet growth. The loss of staurolite and the formation of kyanite-bearing assemblages coincides with renewed garnet growth, such that kyanite-zone samples can be expected to contain a significantly higher modal abundance of garnet.

MAN1 and 24-99 are very similar, both texturally and with respect to their structural setting and tectonometamorphic history. Elevation of the average garnet radius and modal abundance in MAN1 compared to 24-99 likely arose from slight differences in their bulk chemistry, such that the garnet population in MAN1 is predicted to have nucleated at lower $T$ and crystallised over $60{ }^{\circ} \mathrm{C}$, compared to $46{ }^{\circ} \mathrm{C}$ in $24-99$. Given 24-99's slightly higher metamorphic conditions in the IMS, the inverse may otherwise be expected. The early garnet volume production in MS7A is comparable to that of MAN1, and increased radii in the former can be related to the longer $P-T$ interval $\left(95^{\circ} \mathrm{C}\right)$ of garnet growth. Garnet crystallisation in MAN22 has not been constrained, but its large garnet volume is potentially linked to the second phase of garnet growth after the loss of staurolite from the assemblage along a $P-T$ path comparable to others in Sikkim (Fig. 4.16). In each case (and particularly in MAN22), differences in predicted and observed volumes (arrows, Fig. 4.16;) may be partially related to the resorption of garnet in real samples. Continuation of the $P-T$ paths for MAN1, 24-99, and MS7A to kyanite-bearing assemblages indicates that absolute volume percent is expected to differ within these samples at given $P-T$ conditions. These differences are interpreted to derive from slight variations in bulk rock chemistry that differentially stabilize garnet and control the relative timing of garnet-forming reactions.

The thermodynamic model used here is able to predict the observed garnet vol- 
umes very well, and abundances do not deviate from the models more than might be expected for elevated resorption of garnet at higher grades. However, the consistency of observed volumes with those modelled cannot account for the systematic differences in garnet crystal density and size from the garnet to the kyanite zone in all but the SIM samples. In order to explain this trend Carlson (1999) hypothesised that when nucleation occurs at high- $T$, the nucleation of relatively few porphyroblasts (each of which grow rapidly due to high component diffusivities) is enough to maintain equilibrium. Conversely during nucleation at low- $T$, slow rates of transport necessitate significant overstep and nucleation and growth of large number of smaller crystals to maintain equilibrium, resulting in elevated nucleation rates. Such variation might control porphyroblast abundances if there was a correlation between observed grain size/abundance and nucleation $T$ (Carlson, 1999; Hirsch, 2008). However, the modelling indicates that there is no such systematic variation in Sikkim, possibly necessitating the relative loss of small porphyroblasts in high grade samples. George \& Gaidies (2017) also determine low overstep magnitudes at the garnet grade, inconsistent with the Carlson (1999) model. Most importantly, the model cannot account for the expected evolution of microstructure (i.e., a decrease in crystal abundance and a concomitant increase in size) as a sample moves through low-to-high grades.

It seems likely that the decrease in crystal abundance and increase in crystal size, whilst maintaining thermodynamically predicted volumes may be related to progressive volume redistribution due to annealing at high- $T$. Grain coarsening driven by reduction in interfacial energies was invoked by Miyazaki (1991, 1996) to account for changes in porphyroblast size and abundance with grade, and since objections by Carlson (1999), the subject has been much debated. A significant tenant of the argument against Ostwald ripening is based on the assumption of diffusion-controlled garnet growth. However, even in relatively low- $T$ garnets from Sikkim in George \& 
Gaidies (2017), the evidence points to widespread chemical equilibration and crystallisation dominated by an interface-contolled rate law, such that the rate of long-range transport between grains was rapid relative to growth. Thus, diffusion limitation to ripening may not be valid for these rocks. The skewed and often lognormal CSDs measured in this study (Fig. 4.2) are generally comparable to those expected to develop as a result of Ostwald ripening according to Cashman \& Ferry (1988), with the exception of MS7A, which exhibits a linear CSD. Interestingly, garnets in this sample commonly exhibit euhedral surfaces and limited resorption, despite containing some staurolite.

\subsection{Discussion of polycrystalline microstructures in SIM sam- ples}

Along the $P-T$ path for SIM3 in Fig. 4.16a and using bulk compositions of restricted equilibration volumes, Type A and B rock volumes in SIM3 are predicted to contain 5.0 vol. $\%$ and 3.0 vol.\% of garnet, respectively. These predictions are effectively identical to the 5.0 vol.\% and 2.5 vol.\% determined with XR- $\mu$ CT. Moreover, calculation of garnet volume along the same $P-T$ path using instead the SIM3 whole-rock XRF chemical composition predicts a total garnet volume of 4.9 vol.\%, compared to the actual whole rock 4.6 vol.\%. Despite the strongly heterogeneous garnet microstructures that are not generally considered suitable for phase equilibria modelling on account of the apparent lack of textural and chemical equilibration at very local scales, the use of equilibrium thermodynamics is able to very well constrain the average rock volume. Thus, SIM3 must largely differ with respect to the kinetic controls on the distribution of the garnet volume compared to other samples. These may relate to a heterogeneous distribution of nucleation sites and strongly matrix-controlled 
transport-limited growth linked to intrinsic rock properties. The potential delay in the onset of garnet growth relative to deformation may relate to systematic variation in the bulk chemistry in these samples compared to others in the transect.

\subsubsection{Bulk chemical effects on relative timing of porphyroblast nucleation in Sikkim}

As discussed and observed in models presented here, the thermodynamically predicted onset of garnet stability is highly sensitive to bulk rock chemistry (and particularly $\mathrm{MnO}$ content), and thus often accounts for the differences in the relative timing of garnet nucleation and growth in samples that experienced a given thermobarometric history (e.g., Ganguly, 1961; Mahar et al. 1997). However, unlike in Chakraborty et al. (2007), differences in bulk rock MnO do not correlate with pre-/syn- and postkinematic garnet in Sikkim.

Samples containing post-kinematic garnet are consistently enriched in bulk $\mathrm{Na}_{2} \mathrm{O}$ (Table 4.2), and there is a relationship between bulk $\mathrm{Na}_{2} \mathrm{O}$ and the grossular content in garnet cores in these samples, which suggests that the influence of whole-rock $\mathrm{Na}_{2} \mathrm{O}$ on matrix phase relations affected the $T$ (and therefore relative timing) at which garnet grew. Figure 4.17 explores variations in bulk $\mathrm{Na}_{2} \mathrm{O}$ in MAN1 and the bulk relevant to Type A garnet in SIM3. The topologies of both pseudobinary phase diagrams are broadly similar, with a strongly Na-dependent stability of clinozoisiteand biotite-bearing assemblages. In both cases, plagioclase-bearing assemblages are stable over all but the lowest plotted $\mathrm{Na}_{2} \mathrm{O}$ contents in $T-X_{N a}$ space, and contours of plagioclase vol.\% show that plagioclase mode increases significantly with bulk $\mathrm{Na}$ (Fig. 4.17).

The stability of garnet-bearing assemblages can be seen to be subtly influenced by $\mathrm{Na}$ content. At low-Na $(<2 \mathrm{~mol} . \%$ in MAN1 and $<1.7 \mathrm{~mol} . \%$ in SIM3), the $T$ of 
the garnet-in is at a minimum in each given system and takes place in a clinozoisitebearing assemblage. At elevated whole-rock $\mathrm{Na}_{2} \mathrm{O}$, clinozoisite leaves the stable assemblage before the onset of garnet crystallisation, and the $T$ of garnet-in significantly increases. For a range of bulk rock compositions, the high $\mathrm{Na}_{2} \mathrm{O}$ garnet-in $T$ is up to 25-30 ${ }^{\circ} \mathrm{C}$ higher compared to systems with low $\mathrm{Na}_{2} \mathrm{O}$. Along a 5 kbar isobaric path in plagioclase-bearing assemblages at relatively low Na, garnet in MAN1 forms prior to clinozoisite loss and plagioclase abundance varies from 1-2 vol.\% (Fig. 4.17k). As the modal abundance of clinozoisite decreases to high- $T$, Ca liberation and its preferential partitioning into plagioclase yields increasingly anorthitic plagioclase $\left(X_{C a}\right)$, and grossular-poor garnet (Fig. 4.17k). At clinozoisite-out, both plagioclase and garnet decrease in abundance. Along isobaric paths at relatively high whole-rock $\mathrm{Na}_{2} \mathrm{O}$, clinozoisite leaves the stable assemblage at low- $T$ before garnet forms (Fig. 4.17d). While $X_{C a}$ in plagioclase is low at these conditions, the elevated modal abundance of feldspar necessitates increased partitioning of $\mathrm{Ca}$ into plagioclase, manifesting as a negative correlation between garnet grossular content and increasing bulk $\mathrm{Na}_{2} \mathrm{O}$, as is observed across the LHS in Sikkim (Fig. 4.9e).

Irregular suppression of the crystallisation of peraluminous minerals in the chloritebiotite-garnet zones as a result of alkali element (e.g., Na, K, Ca) transfer was first proposed by Chinner (1967) and Hoschek (1969) for the Barrovian type locality. In this case, regional-scale metasomatic transfer of $\mathrm{K}$ and $\mathrm{Na}$ was thought to have yielded a gradient that enriched low-grade samples in alkalis that promoted micaceous crystallisation but inhibited crystallisation of porphyroblastic chloritoid and/or staurolite. While regional-scale fluid flux can often account for transfer of components (e.g., Chinner, 1967, Atherton \& Brotherton, 1982, Ague, 1991, 1994), gradients in Na would be expected to persist to lower grades and inhibit growth of garnet in samples of lower grade than SIM samples if fluid flux had been extensive in Sikkim. Estimates 
of element mobility in Sikkim by Chakraborty et al. (2007) using methods described in Ague (1991) anticipate efficient mass transport of $\mathrm{Na}$ only at the hand-specimen scale, and a comprehensive chemical study of Sikkimese LHS rocks by Dasgupta et al. (2009) indicated that fluid-driven element mobility in Sikkim was relatively low at scales larger than hand specimen scale. Systematic variations in $\mathrm{Na}_{2} \mathrm{O}$ may therefore be derived from primary heterogeneities in the bulk chemistry of the protolith.

The influence of elevated primary $\mathrm{Na}_{2} \mathrm{O}$ in the SIM sequence might therefore serve to delay the average temperature/timing of garnet growth relative to adjacent samples with a lower $\mathrm{Na}_{2} \mathrm{O}$. However, if deformation of the sequence was coherent (or variable but in a coherent manner) elevated $\mathrm{Na}_{2} \mathrm{O}$ cannot account for the full extent of the microstructures, as the $T$ of post-kinematic garnet growth in the upper garnet-lower staurolite zone corresponds to a $P-T$ - $t$ at which progressive deformation was apparently ongoing in adjacent samples (Fig. 4.16). This may provide indirect evidence for the decoupled nature of the SIM sequence.

\subsubsection{Controls on heterogeneous garnet distribution}

Porphyroblastic microstructures (and some chemistries) in SIM3 (Fig. 4.5) are at odds with those generally expected in hydrous and fluid-rich low-medium grade metapelites in which abundant grain boundary fluid facilitates efficient material transfer to reacting sites. Instead, garnet in SIM3 indicate a highly heterogeneous distribution of nucleation sites, preferentially situated at the margin of foliation elements, near or overlapping the interface between heterogeneous quartzo-feldspathic and micaceous domains, and in quartz- and feldspar-rich layers, at odds to proposals that garnet preferentially nucleates in component-rich domains (e.g., Carlson et al., 1995; Carlson, 2011). Garnet microstructures indicate that growth persisted in a highly differential manner, depending on component availability in the immediate vicinity of the sur- 
face. The post-kinematic nature of garnet in SIM3 in a strongly segregated matrix may therefore yield insight into preferential sites of solid-state nucleation in deformed metapelites, and subsequent transport-limited growth controlled by the nature of the matrix schistosity.

The development of spatial domains of significantly differing modal abundances requires some initial segregation of material (DeVore, 1955). Much of this variation may be broadly attributable to primary heterogeneity in the protolith, which in turn yields differential transport kinetics and responses to deformation. The Butanese Paro and Jaishidana sequences with detrital peak ages of 1.80 Ga (McQuarrie et al. 2008) are considered lateral continuations of the units in the Sikkim LHS, and have been interpreted to have been deposited in an active distal foreland basin associated with the Bhimpedian orogeny (McQuarrie et al. 2008). Interbedding of pelites and psammites in this region is consistent with changing sedimentation dynamics in a near-shore environment, with fluctuating input of younger GHS detritus and older Indian Shield detritus (McQuarrie et al., 2014). For the case in which the presence of distinctly different SIM samples is attributable to late, small-scale roof thrust splays, the out-of-sequence units may be a section representative of a slightly different period of sedimentation compared to adjacent regions in IMS.

Chemical gradients derived from original sedimentary layering can drive diffusion and produce segregation layering by the preferential removal of quartz from otherwise micaceous domains (Orville, 1962; Vidale, 1969), and differences in the local stress state, $P$ gradients, and chemical potentials in limb and hinge regions of folds further drive matrix differentiation (e.g., Williams, 1972; Fletcher, 1977). Primary heterogeneities related to sedimentation are thus likely to have been further enhanced by internal metamorphic differentiation of this previously segregated texture (e.g., Williams, 1972; Dewers \& Ortoleva, 1990; Hobbs et al., 2011). Metamorphic differ- 
entiation is generally considered in relation to the development of a $\mathrm{S}_{2}$ crenulation cleavage, but can also enhance $\mathrm{S}_{1}$ (e.g., Talbot \& Hobbs, 1968; Vidale, 1974; Soula \& Debat, 1976).

Alternatively, mass transfer during a period of interaction with a reactive fluid may develop compositional spatial heterogeneities (e.g., Beinlich et al., 2010). While grain boundaries may have been relatively fluid-rich, there is little evidence in SIM3 quartzrich zones for significant precipitation from a fluid prior to garnet crystallisation (e.g., preserved or annealed fluid inclusions), and the preservation of folded micaceous domains through these regions makes it unlikely that the quartz-rich layers are relicts of channelised fluids.

Regardless of the mechanism by which it formed, a major consequence of a strongly layered matrix texture is a highly heterogeneous effective intergranular diffusivity through different mineralogical domains. In general, the grain boundary diffusivity (in a mobile or immobile intergranular fluid phase) is several orders of magnitude faster than volume diffusivities, and at rapid timescales (such as those in Sikkim) and low Ts, dominates mass transfer in the system (Farver \& Yund, 1991, 1992, 1996; Dohmen \& Milke, 2010). Spatial variation in mineral modes and sizes throughout SIM3 will serve to influence the kinetics of crystallisation by altering the effective grain boundary diffusivity in a given matrix domain: in quartz rich domains compared to micaceous domains, the absence of a shape-preferred orientation and relative coarseness of the quartzo-feldspathic phases serves to dramatically increase grain boundary tortuosity and reduce the effective diffusivity (c.f., Kohn \& Penniston-Dorland, 2017). In contrast, the crystallographically preferred orientation in micaceous domains can be expected to serve as rapid channels for grain boundary diffusivity parallel to the [001] cleavage, with intergranular and surface diffusivities further enhanced by the adsorption of $\mathrm{H}_{2} \mathrm{O}$ to mica grain boundaries (Robin, 1979, Holness, 1993; Dempster 
et al., 2017). Dempster et al. (2017) also demonstrated that the diffusivity of divalent cations along quartz-plagioclase boundaries is slow in comparison to other phase and interphase boundaries, prior to the consideration of network tortuosity. In addition, Carlson \& Gordon (2004) showed that for a network comprising interconnected grain edges, effective diffusion coefficients scale with the squared inverse of the matrix grain size. A rock with an average grain diameter of $10 \mu \mathrm{m}$ would therefore have a characteristic lengthscale of diffusion that is 100 times larger and diffusion-controlled growth that is 1000 times faster than in the case of a matrix comprising $1 \mathrm{~mm}$ grains. Thus, when component transfer is controlled predominantly by grain boundary diffusion, supply of materials to reacting sites is likely to be strongly rate-limiting.

\section{Heterogenous distribution of nucleation sites}

The aformentioned strongly layered matrix structure - whether primary or enhanced by metamorphic differentiation - can be seen to have resulted in a strongly heterogeneous distribution of nucleation sites. We tentatively attribute this distribution to a number of major controls on the sites of garnet nucleation: (1) regions of significant chemical heterogeneity in which major and trace element concentrations are favourable for garnet nucleation; (2) epitaxial crystallisation of garnet on mica surfaces in sites of relative component enrichment; and (3) strain-assisted nucleation related to differing strain partitioning in quartz-rich and mica-rich layers.

Epitaxial crystallisation Consistent with the results of others (e.g., Spiess et al., 2007; Cruz, 2011; Moore \& Carlson, 2015), EBSD data presented in this study indicate that some early-nucleated garnet preferentially exploited mica surfaces (Fig. 4.12 ) directly at the boundary between quartz- and mica-rich domains. However, later nucleated garnets do not seem to exploit available equivalent surfaces as might be expected if the energetic aid provided by mica surfaces was a first order control on 
the spatial disposition of garnet, but cluster in a local chemical system adjacent to pre-existing grains. It is interesting to note that these chemical cores are not always in the interior of mica-rich domains, which might be the case if component supply/availability was the predominant control on nucleation sites.

Chemical heterogeneities In addition to potential major element build up at the boundaries between micaceous and quartzose domains, previous assessment of trace element distributions in metapelites have indicated that they are unlikely to be equilibrated across the scale of a hand sample (e.g., Lanzirotti, 1995, Skora et al., 2006; Moore et al., 2013), and SIM3 likely contains a similarly heterogeneous and immobile distribution of trace elements. There is some evidence (George et al., 2018) that variations in the concentration of immobile HREE might create sites of relative nucleation preference, such that sites of elevated HREE facilitate early nucleation. Given the presence of garnet polycrystals in well-defined layers, it may be that these regions inherit primary trace element enrichment.

Strain assisted nucleation The strongly domainal rock texture and modal mineral distributions may have also yielded heterogeneous strain partitioning throughout the rock volume leading to the heterogeneous distribution of dislocations. In deforming quartz-mica fabrics, mica rotates away from the maximum principal stress direction and accommodates deformation along $\{001\}$, so that the stress acting on quartz with boundaries with mica is lesser (Cosgrove, 1972; Bell et al., 1986). Conversely in quartz-rich regions, quartz is increasingly stressed because it is not shielded by fine-grained strain-accommodating mica, accounting for the common observation of porphyroblasts in the hinge regions of crenulation cleavages (Kamb, 1959, 1961; Fletcher, 1977; Dewers \& Ortoleva, 1989; Hobbs et al., 2011). Furthermore, Bell et al. (1986) suggested that a strain gradient can be expected to exist across domain boundaries as a consequence of these differential responses to imposed strain in mi- 
crolithons and cleavage domains, and in the absence of solution transfer at domain boundaries, the highest density of dislocations is expected at the margins of quartz domains.

A key outcome of deformation of segregated matrix domains is therefore that a heterogeneous distribution of dislocations will develop in response to some imposed strain. Here, we propose that the preferential accumulation of dislocations in folded quartz-rich domains due to strain partitioning contributed to the strongly heterogeneous nucleation distribution in SIM samples. As strain energy in dislocations contributes significantly to overcoming the nucleation barrier (e.g., Gaidies, 2017) this may yield regions with significantly differing nucleation potential. The preferential clustering of potentially late-nucleated garnet in Map 2 may indicate that after initial nucleation of a population, the beneficial effect of dislocations exceeds that provided by epitaxial crystallisation on exposed mica phases. The spatial disposition of Type B garnets almost entirely within quartzose domains may indicate that this is a dominant control on nucleation. It is important to note that pre-existing garnet may have played a role in assisting nucleation, but there is no crystallographic evidence for this in this dataset.

While this discussion has pertained to the distribution of porphyroblastic garnet, increased dislocation density near the boundary between micaceous and quartzose domains, coupled with the accumulation of porphyroblast-forming components at this interface, may also account for the preferential distribution of biotite at these margins. Phase equilibria modelling of SIM3 indicates that biotite is expected to become stable directly prior to garnet (Fig. 4.15). Coarse biotite at these margins is generally foliation-parallel, but may have exploited similar nucleation aids as later post-deformation garnet. 


\section{Differential interface- and diffusion-controlled growth}

The growth microstructures in SIM samples might not be out of place in high $P$ and ultra-high $P$ rocks (e.g., Spry, 1969; Stöckhert et al., 1997; Hawkins et al., 2007), but are generally not common in low-medium grade metapelites. They are consistent with strongly heterogeneous garnet growth, such that the combined rates of volume, grain boundary and interphase diffusion were not fast enough to allow for textural or chemical equilibration across matrix layers, and even along surfaces of some garnet grains.

Along euhedral interfaces in coalesced polycrystals, the crystallographically-controlled garnet morphology and the equilibration of garnet chemistry along the rim may indicate that diffusion of garnet-forming components to the surface during growth was rapid, yielding a garnet chemistry that was controlled by changing equilibria through $P-T$ space.

Conversely both the chemistry and morphology of interfaces in contact with quartzose domains is strongly space-dependent and is not broadly equilibrated. Type A garnet in SIM3 often protrudes along quartz-quartz grain boundaries and sometimes forms thin continuous films along these boundaries, providing additional microstructural evidence for the influence of transport along grain boundaries. Growth of these surfaces was demonstrably slower and was limited by the relative availability of components, such that the rate of growth was controlled by the relative local rate of transport to and along the surface. Unlike honeycomb garnet in Hawkins et al. (2007), microstructures are not consistent with direct precipitation from a wetting siliceous aqueous fluid, but the inference of transport-controlled growth is in line with interpretation of irregular morphologies in Spear \& Daniel (2001) and Miyazaki (2015). Grain boundaries may have served to facilitate the formation diffusional instabilities across

the surface of growing garnet (Miyazaki, 2015), such that more rapid growth rates 
in the vicinity of these regions led to the development of branching microstructures that were not inhibited by interfacial energies. Growth of garnet in quartz-rich layers likely prevented the effective development of chemical potential gradients of $\mathrm{SiO}_{2}$ and limiting diffusive displacement of quartz (Foster, 1982), resulting in the incorporation of quartz inclusions proximal to some transport-controlled surfaces (Fig 4.5), and the cessation of growth of garnet at mica-quartz boundaries (e.g., in Map 1). Early cessation of growth in these regions is also likely to be linked to the relative absence of $\mathrm{Al}$, commonly considered to be rate-limiting due to its slow diffusivity and relative insolubility in metamorphic fluids (e.g., Ashworth et al., 1992; Carlson et al., 1995). In the case of transport-controlled garnet growth moderated by component supply along grain boundaries, gradients in the concentrations of rate-limiting components might be expected to propagate away from the garnet surface.

\subsection{Conclusions}

These data document that the systematic correlation first documented by Cashman \& Ferry (1988), between metamorphic grade and porphyroblastic garnet microstructure, exists through coherent and continuous portions of the IMS in the Sikkim Himalaya. Aside from the correlation of radii with peak $T$, phase equilibria and garnet crystallisation modelling of samples along these sections highlights the role that subtle variations in bulk composition play in controlling absolute garnet volume, through resultant changes in the $T$ of initial garnet crystallisation and relative reaction histories. The modelled $T$ of incipient garnet crystallisation does not vary systematically with observed garnet size, so a model in which the crystal size-density correlation arises as a result of systematically changing nucleation $T$ s is not supported. Instead, garnet microstructures are potentially consistent with having developed during ripening at 
progressively elevated $T$ s.

Deviations from this systematic trend in the form of strongly heterogeneous and primary polycrystalline garnet microstructures in chemically distinct rocks at the garnet-staurolite grade point to discontinuities in the sequence, such that the metamorphic evolution of this region was kinematically and/or thermally decoupled from the rest of the exposed IMS. However, despite the presence of distinct garnet populations across the hand sample scale (which are interpreted to have arisen as a consequence of differences in the relative timing of nucleation in localised chemical systems), phase equilibria approaches are able to effectively reproduce absolute garnet volumes and chemically equilibrated rim compositions along a $P-T$ path comparable those of the rest of the IMS. This lends support to the idea of metamorphism of the Sikkim IMS as a coherent block, with subsequent inversion and minor thrustaccommodated tectonics, such that the irregular sequence represents a late-developed out-of-sequence slice.

The ability of the equilibrium model to predict absolute garnet volumes in samples containing evidence diffusion-controlled garnet growth indicates the approach is valuable even for a strongly heterogeneous rock volume in which the rate of transport was rate-limiting for some garnet growth. Assumptions inherent to this approach do not consider the development of porphyroblast microstructure, which, as samples containing post-kinematic garnet in this study show, is strongly dependent on the nature of the matrix at the time of porphyroblast crystallisation even if the rock can be considered broadly chemically equilibrated. The distribution of favourable nucleation sites is a highly complex interplay between heterogeneous chemical distributions at a local scale, epitaxial crystallisation, and strain-assisted nucleation. The layered matrix microstructure shows evidence for the influence of transport-controlled garnet growth compared to interface-controlled garnet growth in the case where effective 
diffusivities of rate-limiting components were slow relative to $P-T-t$ changes. While these principal variables are likely to influence microstructures in the other studied samples, the irregular samples highlight the value of non-typical morphologies in progressing our understanding of porphyroblast crystallisation. 


\section{Tables and Figures}



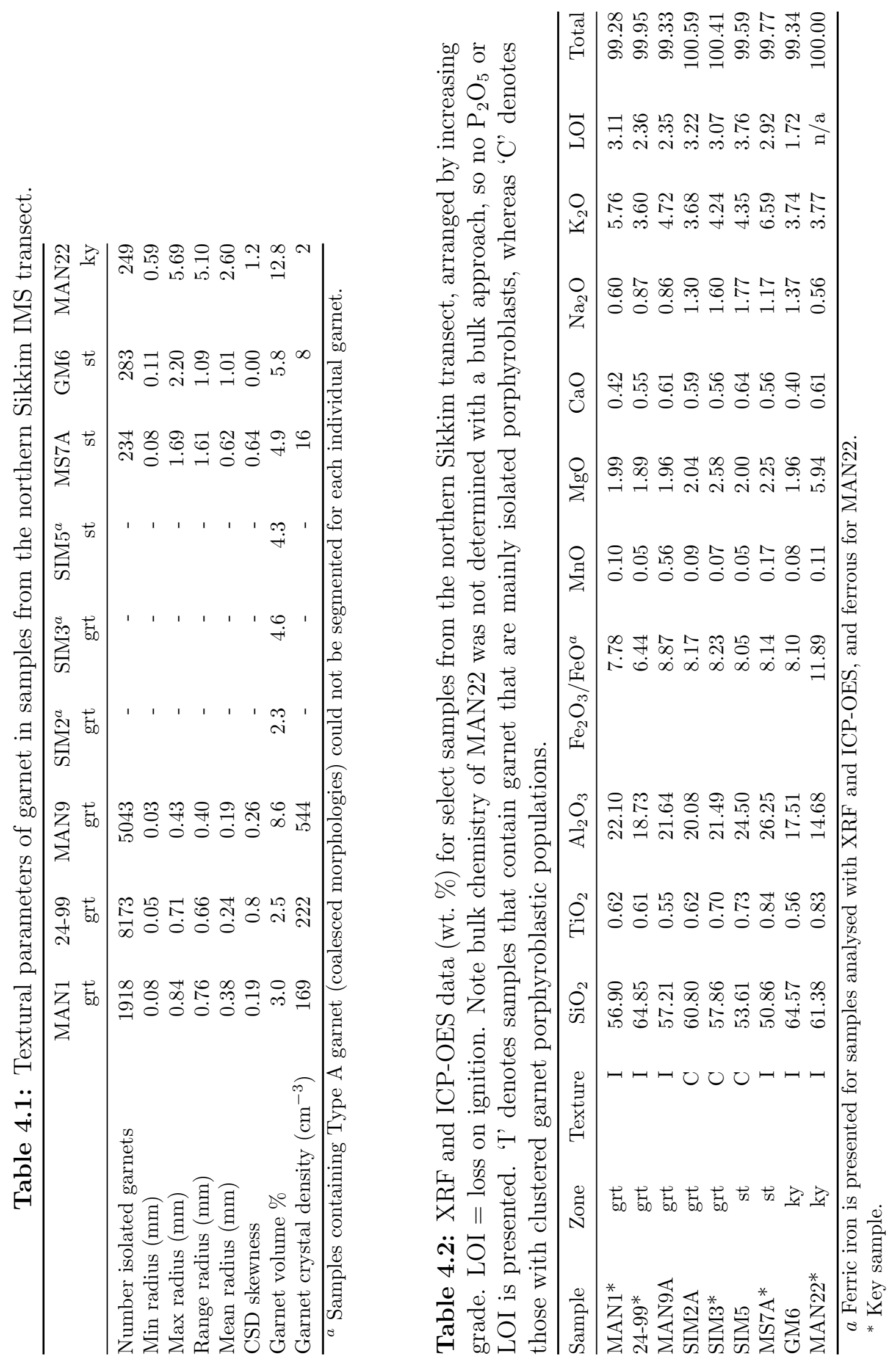
Table 4.3: Modal phase proportions (volume \%) of samples studied, determined by integrating petrographic and XR- $\mu \mathrm{CT}$ analyses.

\begin{tabular}{lcccccccccc}
\hline Phase & Ms & Bt & Qz & Pl & Chl & Grt & St & Ky & Ilm & Acc \\
\hline MAN1 & 49 & 14 & 24 & 1 & 7 & 3.0 & - & - & 0.9 & $<1$ \\
24-99 & 38 & 9 & 33 & 6 & 9 & 2.4 & - & - & 1.0 & $<1$ \\
SIM3 & 32 & 10 & 41 & 8 & 2 & 4.6 & - & - & 1.5 & $<1$ \\
MS7A & 38 & 26 & 21 & 4 & 1 & 4.9 & 2.2 & - & 1.0 & $<1$ \\
MAN22 & 24 & 28 & 24 & $<1^{*}$ & 4 & 12.8 & - & 6 & 0.5 & $<1$ \\
\hline
\end{tabular}

* Plagioclase in MAN22 only present as inclusions in garnet. 


\begin{tabular}{|c|c|c|c|c|c|c|c|c|c|c|c|c|c|}
\hline \multirow{4}{*}{$\begin{array}{l}\text { Sample } \\
\text { Phase } \\
\text { Location } \\
\text { Number }\end{array}$} & \multicolumn{6}{|c|}{ MAN1 } & \multicolumn{7}{|c|}{ 24-99 } \\
\hline & $\mathrm{Bt}$ & $\mathrm{Wm}$ & Ilm & $\mathrm{CzO}$ & Chl & Chl & $\mathrm{Bt}$ & $\mathrm{Wm}$ & $\mathrm{Pl}$ & $\mathrm{Ilm}$ & Ilm & $\mathrm{Chl}$ & $\mathrm{Chl}$ \\
\hline & $\mathrm{Mx}$ & Mtx & $M x$ & Grt & Ret & Pro & $M x$ & Mx & $M x$ & Grt & $M x$ & Pro & Ret \\
\hline & 10 & 8 & 4 & 2 & 4 & 6 & 12 & 6 & 9 & 4 & 2 & 4 & 4 \\
\hline $\mathrm{SiO}_{2}$ & 35.45 & 46.71 & 0.34 & 38.47 & 24.23 & 24.36 & 36.20 & 47.70 & 63.99 & 0.11 & 0.03 & 24.90 & 24.80 \\
\hline $\mathrm{TiO}_{2}$ & 1.70 & 0.28 & 52.30 & 0.12 & 0.08 & 0.10 & 1.52 & 0.25 & 0.00 & 51.54 & 52.29 & 0.09 & 0.10 \\
\hline $\mathrm{Al}_{2} \mathrm{O}_{3}$ & 18.28 & 35.02 & 0.30 & 27.03 & 21.86 & 22.19 & 18.14 & 36.20 & 22.77 & 0.11 & 0.11 & 22.68 & 21.90 \\
\hline $\mathrm{FeO}$ & 21.95 & 1.51 & 44.70 & 7.40 & 30.11 & 29.69 & 21.11 & 0.89 & 0.04 & 46.67 & 46.80 & 26.87 & 28.37 \\
\hline $\mathrm{MnO}$ & 0.05 & 0.01 & 0.84 & 0.48 & 0.11 & 0.10 & 0.03 & 0.01 & 0.00 & 0.40 & 0.40 & 0.04 & 0.05 \\
\hline $\mathrm{MgO}$ & 7.45 & 0.72 & 0.02 & 0.02 & 10.95 & 10.83 & 8.43 & 0.58 & 0.00 & 0.02 & 0.02 & 12.97 & 12.27 \\
\hline $\mathrm{CaO}$ & 0.01 & 0.00 & 0.00 & 22.73 & 0.01 & 0.01 & 0.00 & 0.00 & 3.82 & 0.02 & 0.02 & 0.03 & 0.03 \\
\hline $\mathrm{Na}_{2} \mathrm{O}$ & 0.13 & 1.04 & 0.00 & 0.01 & 0.00 & 0.01 & 0.26 & 1.79 & 9.52 & 0.00 & 0.00 & 0.07 & 0.05 \\
\hline $\mathrm{K}_{2} \mathrm{O}$ & 9.13 & 9.79 & 0.21 & 0.00 & 0.05 & 0.14 & 8.83 & 8.63 & 0.08 & 0.00 & 0.00 & 0.04 & 0.10 \\
\hline Total & 94.15 & 95.08 & 98.71 & 96.26 & 87.39 & 87.41 & 94.51 & 96.06 & 100.23 & 98.87 & 99.67 & 87.70 & 87.67 \\
\hline $\mathrm{Si}$ & 2.76 & 3.11 & 0.01 & 6.17 & 2.63 & 2.64 & 2.79 & 3.11 & 2.82 & 0.00 & 0.00 & 2.64 & 2.66 \\
\hline $\mathrm{Ti}$ & 0.10 & 0.01 & 1.00 & 0.01 & 0.01 & 0.01 & 0.09 & 0.01 & 0.00 & 0.99 & 1.00 & 0.01 & 0.01 \\
\hline $\mathrm{Al}$ & 1.68 & 2.75 & 0.01 & 5.11 & 2.80 & 2.83 & 1.65 & 2.78 & 1.18 & 0.00 & 0.00 & 2.84 & 2.77 \\
\hline $\mathrm{Fe}^{2+}$ & 1.43 & 0.08 & 0.95 & 0.99 & 2.74 & 2.69 & 1.36 & 0.05 & 0.00 & 0.99 & 0.99 & 2.38 & 2.56 \\
\hline Mn & 0.00 & 0.00 & 0.02 & 0.07 & 0.01 & 0.01 & 0.00 & 0.00 & 0.00 & 0.01 & 0.01 & 0.00 & 0.00 \\
\hline $\mathrm{Mg}$ & 0.86 & 0.07 & 0.00 & 0.01 & 1.77 & 1.75 & 0.97 & 0.06 & 0.00 & 0.01 & 0.00 & 2.05 & 1.94 \\
\hline $\mathrm{Ca}$ & 0.00 & 0.00 & 0.00 & 3.91 & 0.00 & 0.00 & 0.00 & 0.00 & 0.18 & 0.00 & 0.00 & 0.00 & 0.00 \\
\hline $\mathrm{Na}$ & 0.02 & 0.13 & 0.00 & 0.00 & 0.00 & 0.00 & 0.03 & 0.23 & 0.81 & 0.00 & 0.00 & 0.02 & 0.01 \\
\hline K & 0.91 & 0.88 & 0.01 & 0.00 & 0.01 & 0.02 & 0.85 & 0.72 & 0.00 & 0.00 & 0.00 & 0.01 & 0.01 \\
\hline Sum & 7.76 & 6.99 & 1.99 & 16.26 & 9.96 & 9.95 & 7.74 & 6.96 & 5.00 & 2.00 & 2.00 & 9.94 & 9.96 \\
\hline Oxygen & 11 & 11 & 3 & 25 & 14 & 14 & 11 & 11 & 8 & 3 & 3 & 14 & 14 \\
\hline $\mathrm{X}_{M g}$ & 0.39 & 0.38 & - & 0.37 & 0.39 & 0.39 & 0.42 & 0.54 & 0.00 & - & - & 0.46 & 0.43 \\
\hline $\mathrm{X}_{P a r}$ & - & 0.14 & - & - & - & - & - & 0.24 & - & - & - & - & - \\
\hline
\end{tabular}

\begin{tabular}{|c|c|c|c|c|c|c|c|c|c|c|c|c|}
\hline \multirow{4}{*}{$\begin{array}{l}\text { Sample } \\
\text { Phase } \\
\text { Location } \\
\text { Number }\end{array}$} & \multicolumn{5}{|c|}{ MS7A } & \multicolumn{7}{|c|}{ MAN22 } \\
\hline & $\mathrm{Bt}$ & $\mathrm{Wm}$ & $\mathrm{Pl}$ & $\mathrm{Ilm}$ & $\mathrm{St}$ & $\mathrm{Bt}$ & $\mathrm{Bt}$ & $\mathrm{Wm}$ & $\mathrm{Pl}$ & Ilm & Chl & Ky \\
\hline & $\mathrm{Mx}$ & $\mathrm{Mx}$ & $\mathrm{Mx}$ & $M x$ & $\mathrm{Mx}$ & $M x$ & Grt & Mx & Grt & $\mathrm{Mx} / \mathrm{Grt}$ & Ret & $\mathrm{Mx}$ \\
\hline & 6 & 4 & 6 & 1 & 2 & 4 & 2 & 7 & 3 & 6 & 5 & 2 \\
\hline $\mathrm{SiO}_{2}$ & 35.81 & 46.27 & 64.88 & 0.07 & 27.52 & 35.70 & 36.50 & 46.10 & 62.18 & 0.02 & 24.57 & 37.15 \\
\hline $\mathrm{TiO}_{2}$ & 1.70 & 0.59 & 0.00 & 53.06 & 0.72 & 1.93 & 1.83 & 0.58 & 0.00 & 50.86 & 0.10 & 0.00 \\
\hline $\mathrm{Al}_{2} \mathrm{O}_{3}$ & 19.10 & 36.16 & 22.82 & 0.03 & 52.56 & 19.28 & 19.12 & 35.94 & 24.07 & 0.08 & 23.37 & 63.76 \\
\hline $\mathrm{FeO}$ & 18.60 & 1.22 & 0.03 & 45.55 & 14.93 & 18.55 & 16.78 & 1.09 & 0.23 & 46.12 & 24.19 & 0.26 \\
\hline $\mathrm{MnO}$ & 0.06 & 0.10 & 0.00 & 0.36 & 0.15 & 0.04 & 0.01 & 0.01 & 0.00 & 0.67 & 0.08 & 0.00 \\
\hline $\mathrm{MgO}$ & 10.53 & 0.57 & 0.00 & 0.21 & 1.75 & 9.83 & 10.89 & 0.66 & 0.00 & 0.26 & 14.91 & 0.02 \\
\hline $\mathrm{CaO}$ & 0.00 & 0.00 & 3.65 & 0.01 & 0.01 & 0.01 & 0.00 & 0.00 & 4.92 & 0.00 & 0.00 & 0.00 \\
\hline $\mathrm{Na}_{2} \mathrm{O}$ & 0.50 & 1.75 & 9.51 & 0.00 & 0.01 & 0.36 & 0.56 & 1.49 & 9.21 & 0.00 & 0.01 & 0.00 \\
\hline $\mathrm{K}_{2} \mathrm{O}$ & 8.56 & 8.40 & 0.07 & 0.00 & 0.01 & 9.21 & 8.75 & 9.23 & & 0.00 & 0.02 & 0.00 \\
\hline Total & 94.85 & 94.97 & 100.97 & 99.29 & 97.64 & 94.91 & 94.44 & 95.10 & 100.70 & 98.01 & 87.24 & 101.20 \\
\hline $\mathrm{Si}$ & 2.71 & 3.06 & 2.83 & 0.00 & 7.70 & 2.71 & 2.75 & 3.06 & 2.74 & 0.00 & 2.58 & 0.99 \\
\hline $\mathrm{Ti}$ & 0.10 & 0.03 & 0.00 & 1.01 & 0.15 & 0.11 & 0.10 & 0.03 & 0.00 & 0.99 & 0.01 & 0.00 \\
\hline $\mathrm{Al}$ & 1.71 & 2.82 & 1.17 & 0.00 & 17.35 & 1.73 & 1.70 & 2.81 & 1.25 & 0.00 & 2.90 & 2.01 \\
\hline $\mathrm{Fe}^{2+}$ & 1.18 & 0.07 & 0.00 & 0.96 & 3.50 & 1.18 & 1.06 & 0.06 & 0.01 & 1.00 & 2.13 & 0.01 \\
\hline Mn & 0.00 & 0.00 & 0.00 & 0.01 & 0.04 & 0.00 & 0.00 & 0.00 & 0.00 & 0.01 & 0.01 & 0.00 \\
\hline $\mathrm{Mg}$ & 1.91 & 0.06 & 0.00 & 0.01 & 0.73 & 1.11 & 1.22 & 0.07 & 0.00 & 0.01 & 2.34 & 0.00 \\
\hline $\mathrm{Ca}$ & 0.00 & 0.00 & 0.17 & 0.00 & 0.00 & 0.00 & 0.00 & 0.00 & 0.23 & 0.00 & 0.00 & 0.00 \\
\hline $\mathrm{Na}$ & 0.07 & 0.22 & 0.80 & 0.00 & 0.01 & 0.05 & 0.08 & 0.19 & 0.79 & 0.00 & 0.00 & 0.00 \\
\hline K & 0.83 & 0.71 & 0.00 & 0.00 & 0.00 & 0.89 & 0.84 & 0.79 & 0.01 & 0.00 & 0.00 & 0.00 \\
\hline Sum & 7.79 & 6.97 & 4.99 & 1.99 & 29.47 & 7.79 & 7.76 & 6.99 & 5.03 & 2.01 & 9.96 & 3.00 \\
\hline Oxygen & 11 & 11 & 8 & 3 & 46 & 11 & 11 & 11 & 8 & 3 & 14 & 5 \\
\hline $\mathrm{X}_{M g}$ & 0.49 & 0.45 & - & - & 0.17 & 0.49 & 0.54 & 0.51 & - & - & 0.52 & 0.10 \\
\hline $\mathrm{X}_{\text {Par }}$ & - & 0.26 & - & - & - & - & - & 0.20 & - & - & - & - \\
\hline
\end{tabular}

Table 4.4: Mineral compositions for samples 24-99, MAN1, MS7A, MAN22 and SIM3. Mtx $=$ matrix mineral, Grt $=$ included in garnet, Pro $=$ texturally prograde, Ret $=$ texturally retrograde, $\mathrm{X}_{M g}=[M g / M g+F e]$ and $\mathrm{X}_{P a r}=[N a / N a+K+C a]$. 


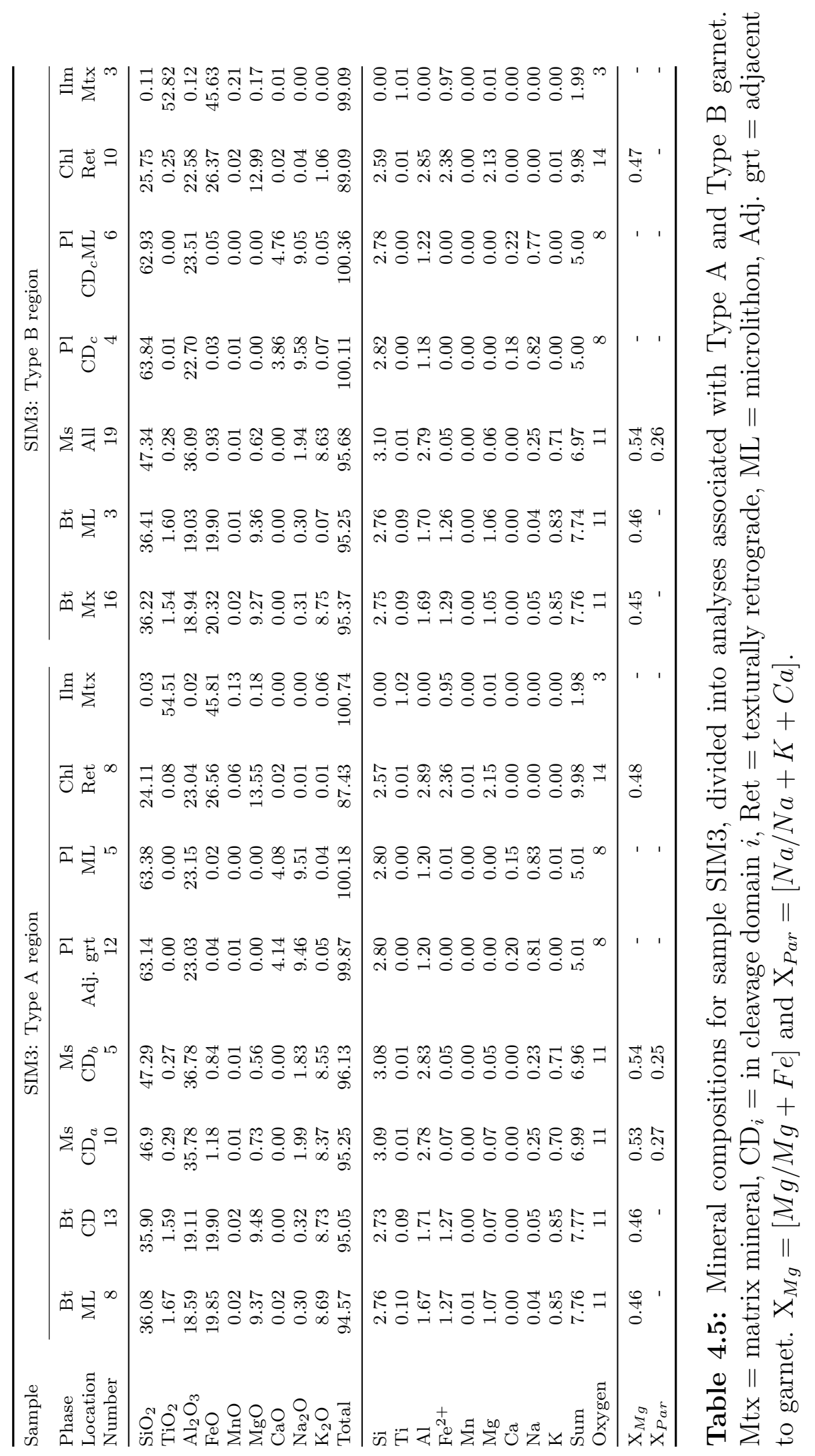




\begin{tabular}{lccccc}
\hline \multirow{2}{*}{ Crystal } & Ilm c-axis, $I$ & Pole to foliation, $F$ & Best fit grt/mica & \multicolumn{2}{c}{ Angle between poles } \\
\cline { 5 - 6 } & & & & $\Delta_{I-x}$ & $\Delta_{F-x}$ \\
\hline 1 & $164 / 43$ & $141 / 11$ & $150 / 28$ & 18.8 & 19.0 \\
2 & $316 / 08$ & $113 / 10$ & $109 / 23$ & 40.7 & 13.6 \\
3 & $\mathrm{n} / \mathrm{a}$ & $100 / 10$ & $078 / 33$ & $\mathrm{n} / \mathrm{a}$ & 30.6 \\
4 & $222 / 34$ & $074 / 10$ & $079 / 30$ & 72.7 & 20.5 \\
5 & $036 / 62$ & $081 / 10$ & $064 / 03$ & 17.7 & 18.0 \\
6 & $256 / 10$ & $261 / 10$ & $073 / 34$ & 43.8 & 44.7 \\
7 & $281 / 01$ & $085 / 10$ & $235 / 03$ & 19.0 & 32.6 \\
8 & $254 / 03$ & $099 / 11$ & $101 / 12$ & 66.1 & 2.20 \\
\hline
\end{tabular}

Table 4.6: EBSD epitaxy measurements corresponding to Fig. 4.12. All angles in degrees. 


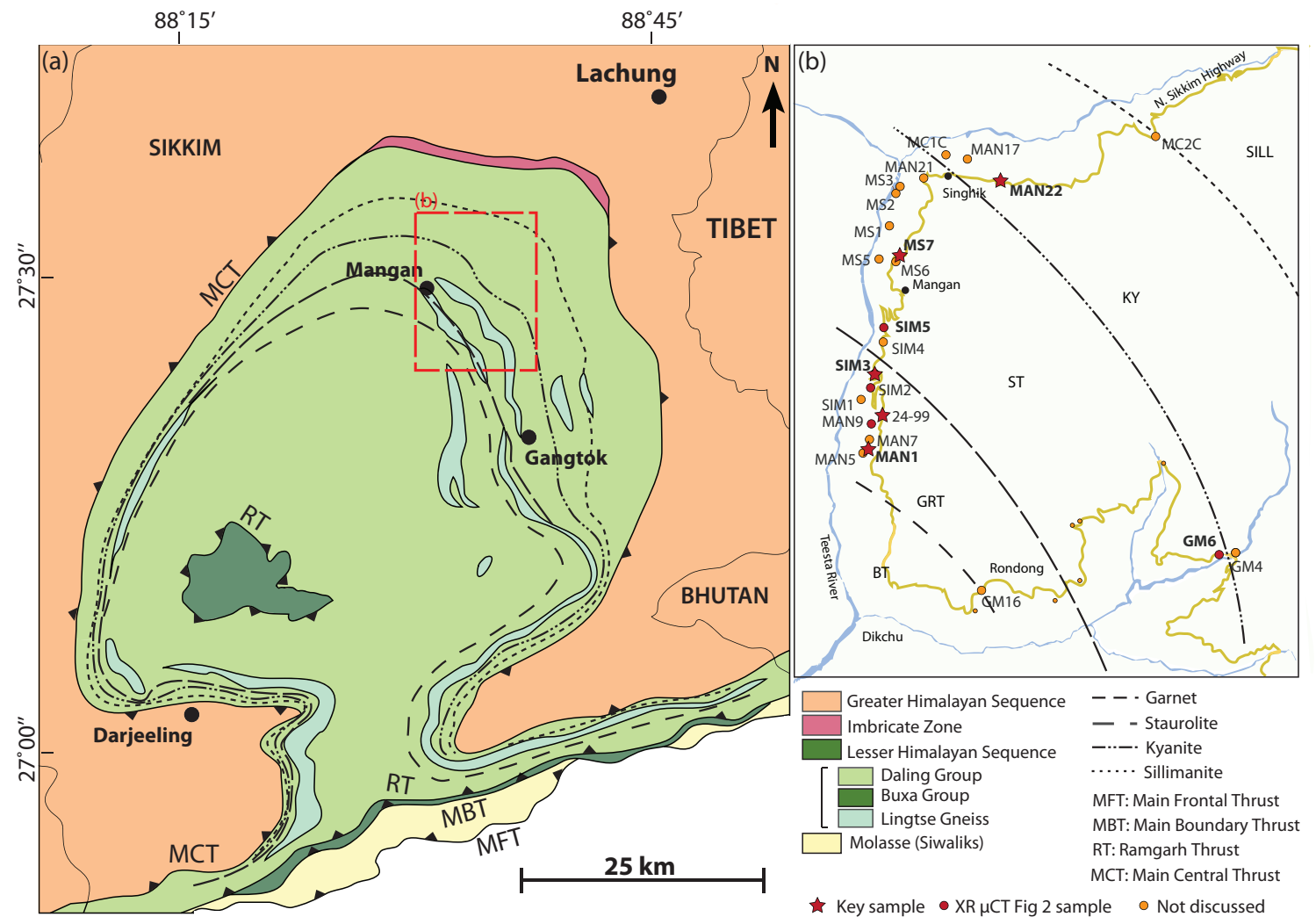

Figure 4.1: Maps of the Sikkim Himalaya. (a) Geological map of the Sikkim Teesta dome, modified after Chakraborty et al (2016). (b) Map of sample locations along the northern transect through the inverted Barrovian sequence. Key samples discussed in this study are denoted with red stars, additional samples presented in Fig. 4.2 in red circles. Approximate location of isograds plotted.

Figure 4.2 (following page): XR- $\mu \mathrm{CT}$ reconstructions and accompanying CSD's (when relevant) of representative porphyroblastic samples from the northern Sikkim inverted Barrovian sequence transect. (a-e) Garnet zone samples MAN1, 24-99, MAN9, SIM2 and SIM3, respectively. (f-g) Staurolite zone samples SIM5 and MS7A. (h) Kyanite-bearing MAN22. Samples in (g-f) exhibit clustered/coalesced garnet morphologies. The largest and centrally-sectioned garnet in each sample is highlighted. Relevant garnet population textural parameters are included in Table 4.1. 


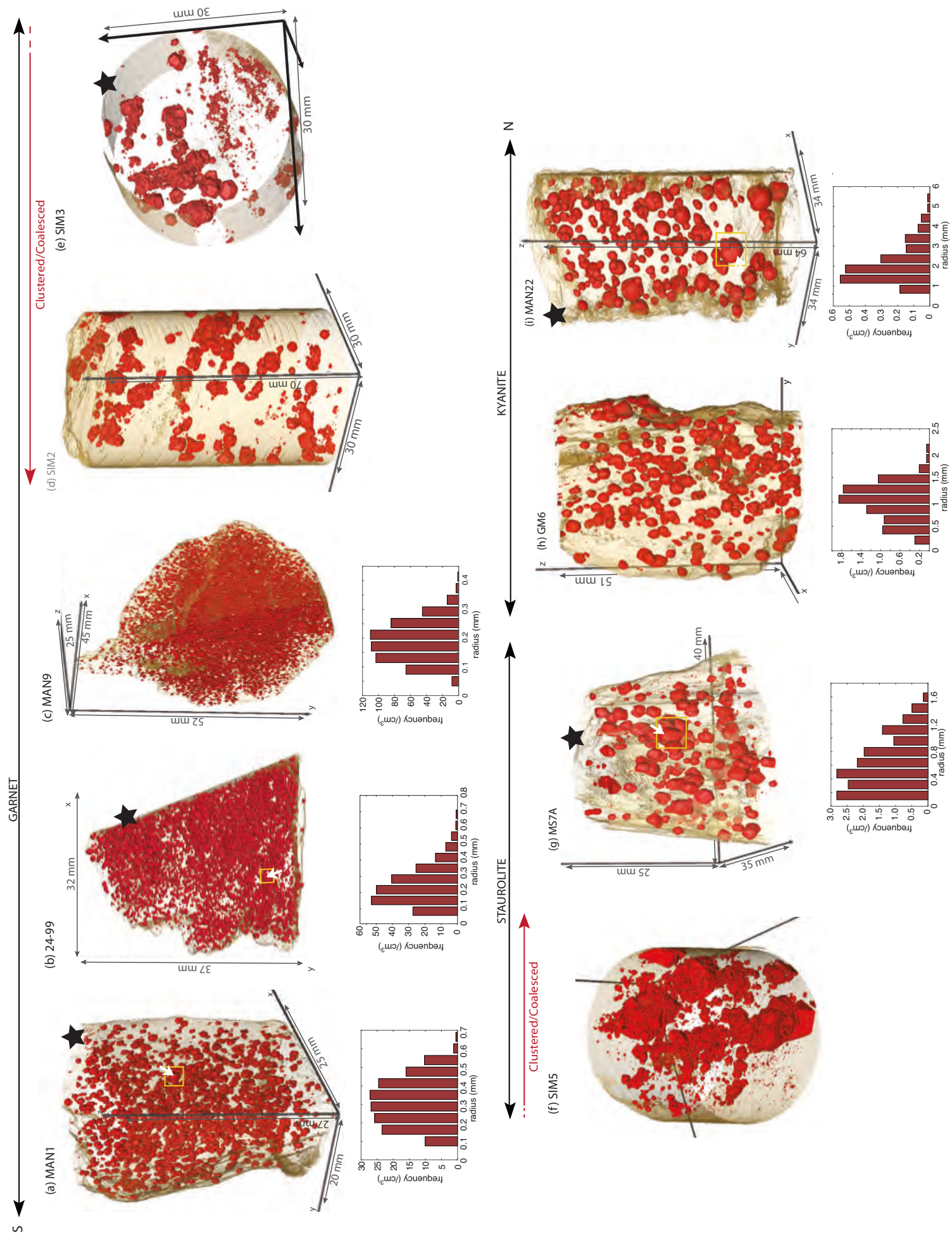



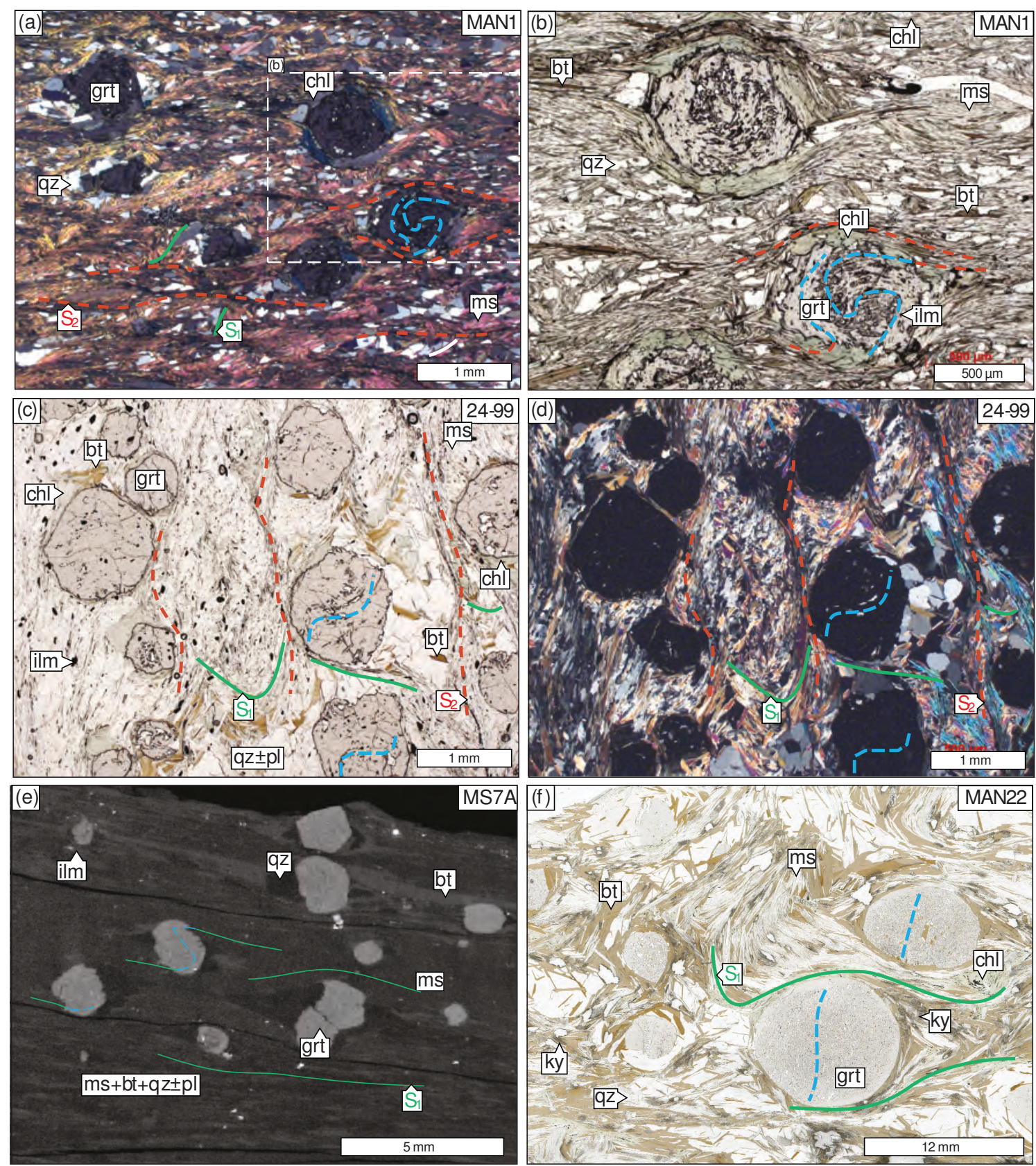

Figure 4.3: Petrography of metepelites assessed in this study. (a) and (b) Plane-polarized and cross-polarized photomicrographs of sample MAN1. Garnet is syn-kinematic and exhibits chlorite strain caps parallel to external $\mathrm{S}_{2}$ crenulation. Quartz is evenly disseminated throughout the matrix. (c) and (d) Plane-polarized and cross-polarized photomicrographs of sample 24-99, containing garnet that is syn-kinematic with respect to $\mathrm{S}_{2}$ deformation, and ragged retrograde chlorite. (e) Calculated XR- $\mu \mathrm{CT}$ slice of MS7A. (f) Partial thin section scan of MAN22 showing large inclusion-rich pre-kinematic garnets in pervasively deformed $\mathrm{S}_{1}$ foliation. Kyanite parallels matrix $\mathrm{S}_{1}$. 
(a) MAN1
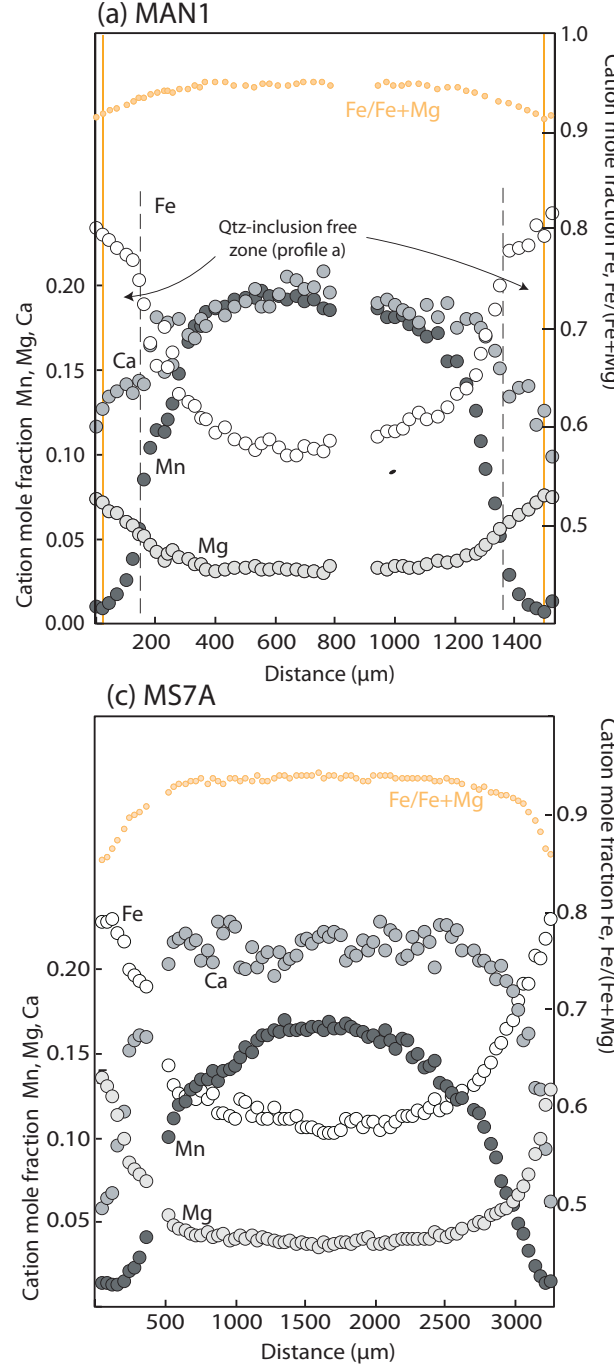

(b) 24-99

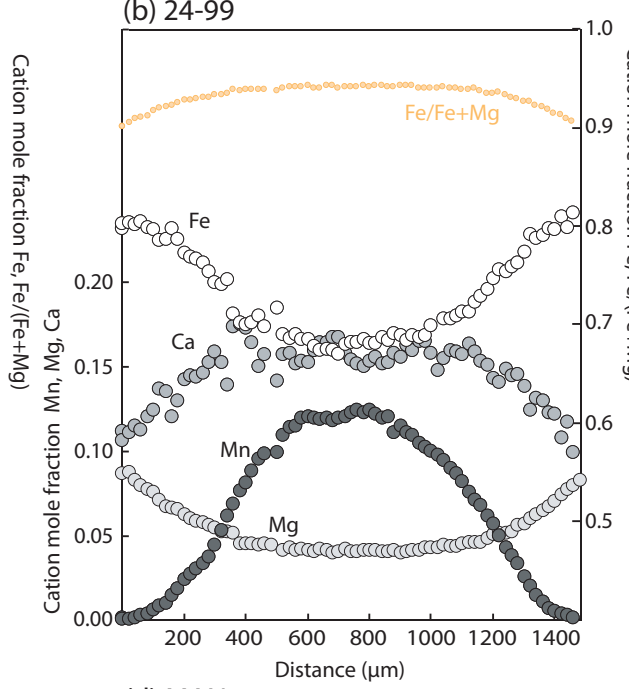

(d) MAN22

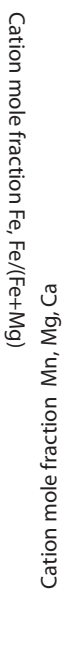

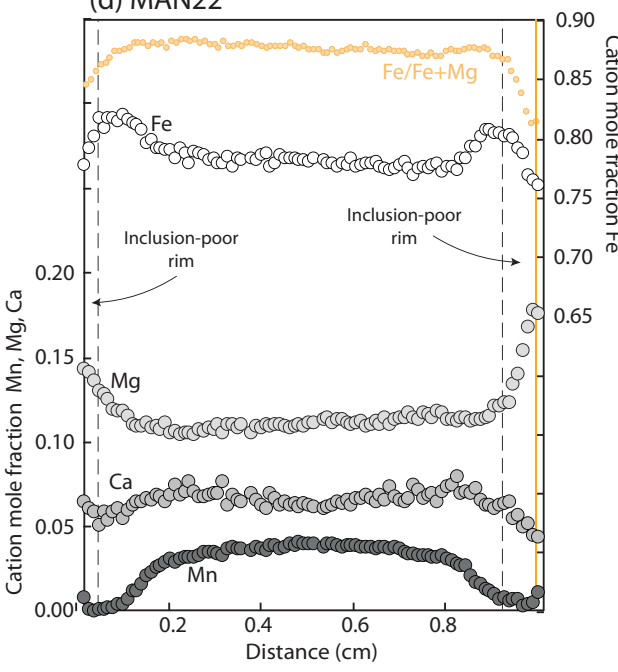
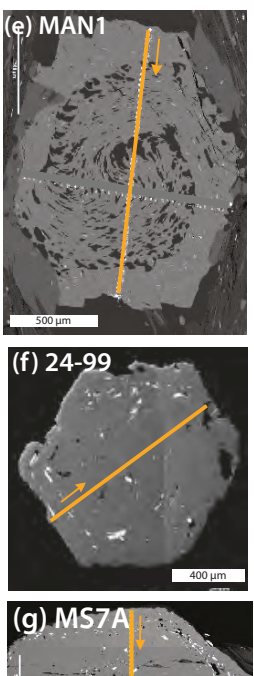

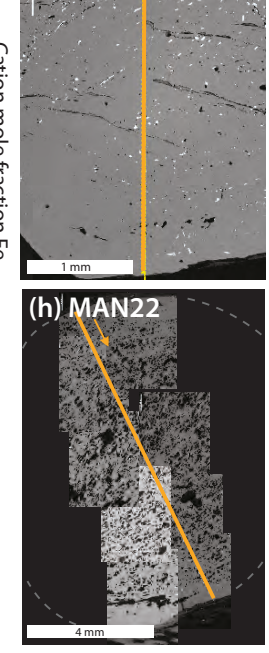

Figure 4.4: Major element compositional line profiles across centrally-sectioned garnet cores. (a-d) Rim-to-rim traverses for samples MAN1, 24-99, MS7A and MAN22, respectively. Vertical orange line denotes limit of chemically altered rim, and dashed line marks the textural transition from inclusion-rich core to inclusion-poor rim. (e-h) Corresponding backscattered EPMA images, showing exact garnet profile locations. 
Figure 4.5 (following page): (a) Calculated XR $\mu \mathrm{m}$-CT slice of SIM3, corresponding to reconstruction in Fig. 4.2 e. Regions corresponding to zones of Type A and $\mathrm{B}$ garnet highlighted; darkest grey predominantly quartz \pm plagioclase, middle grey are cleavage domains, and brightest grey is garnet. (b) Plane-polarised photomicrograph showing differential garnet interface morphology on quartz-rich and mica-rich domains. (c) Quartz-plate photomicrograph showing heterogeneous quartz grain size and variable garnet interfaces. (d) Partial thin section scan of showing broad sample morphology, with locations of Figs. 4.63 and 4.11 and Fig. 4.10 highlighted. (e) Crosspolarised photomicrograph with quartz plate highlighting differential garnet growth on micaceous and quartzofeldspathic domains. (f) Plane-polarised photomicrographs of Type A garnet. 

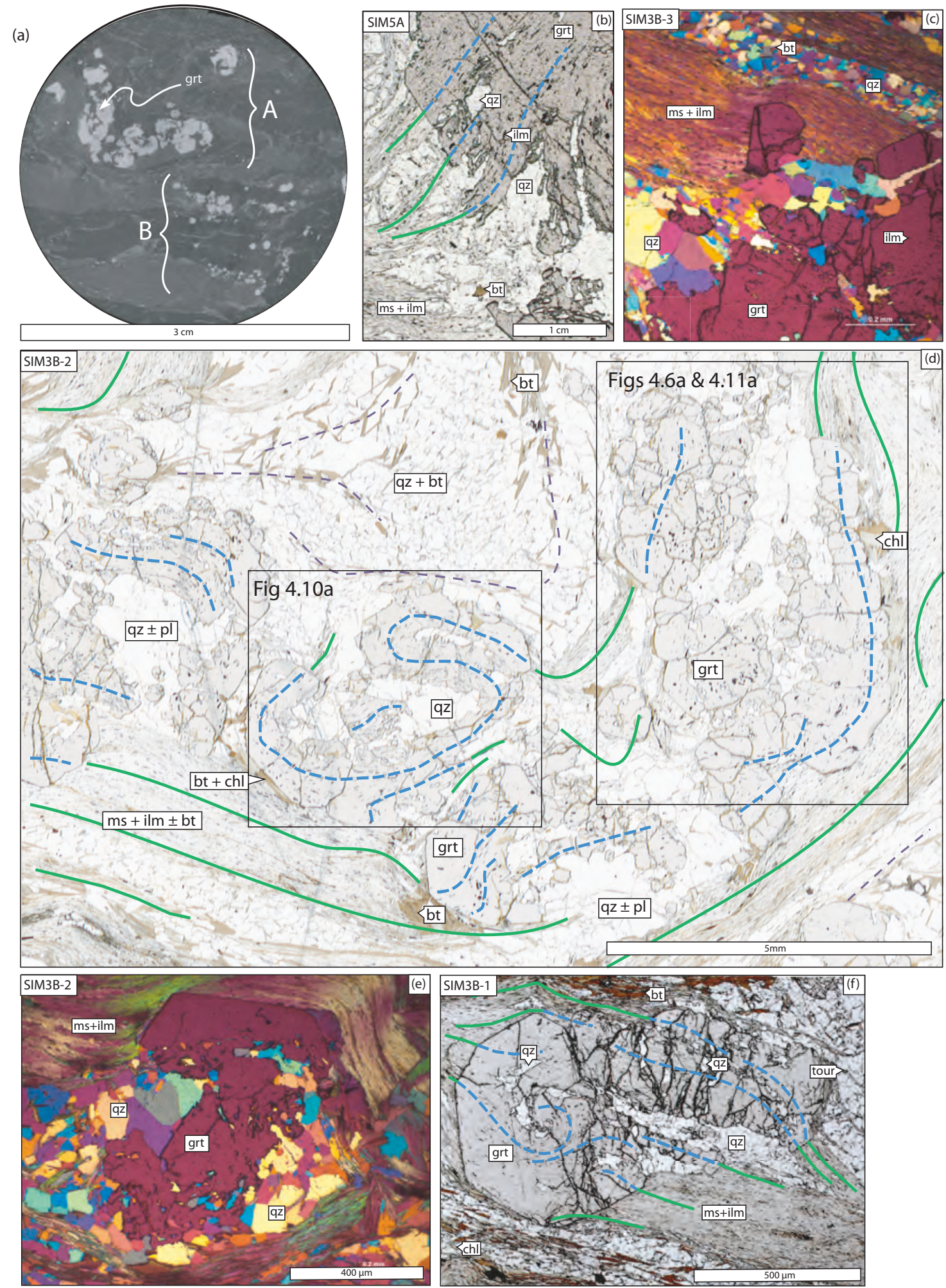

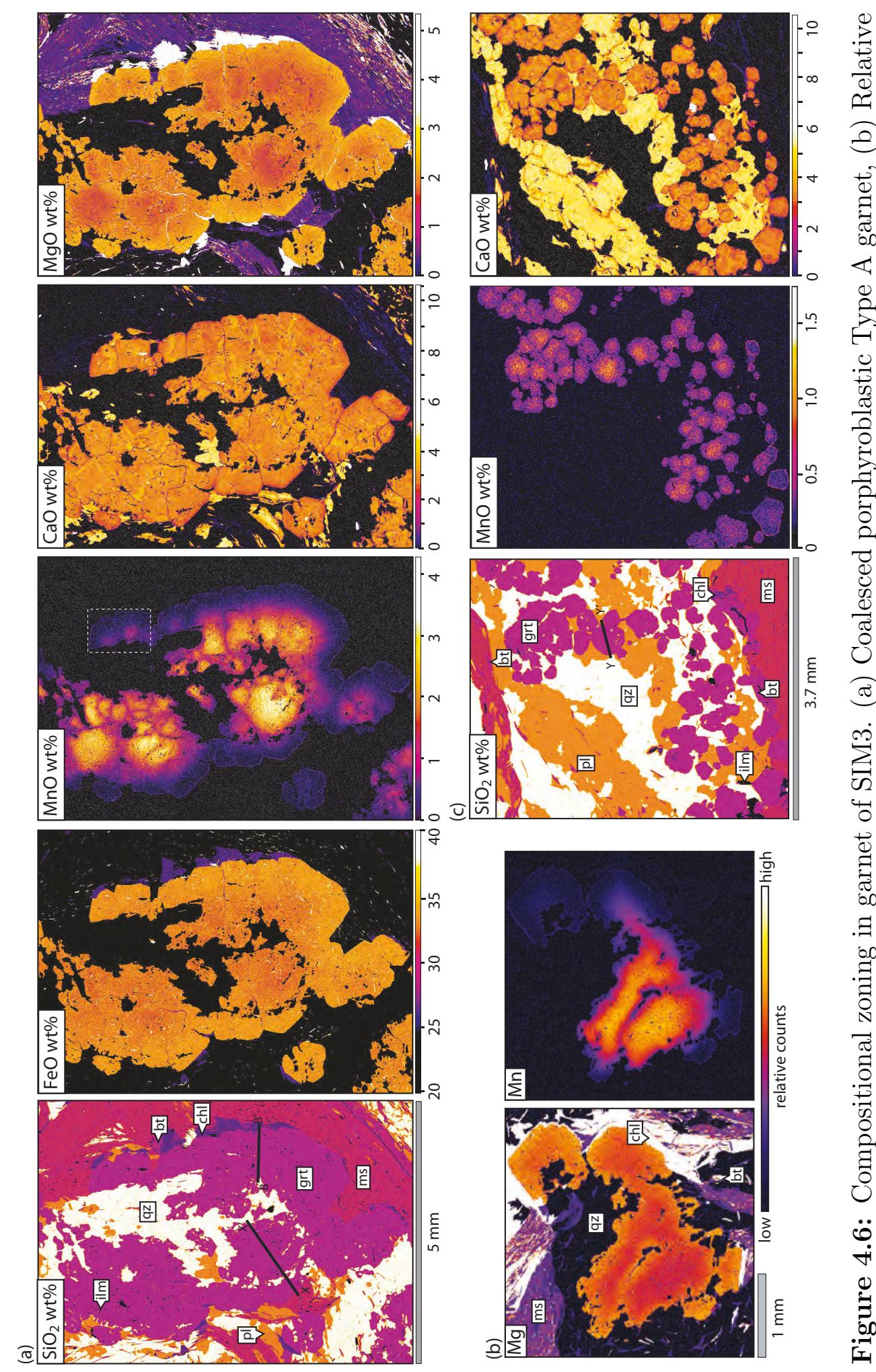

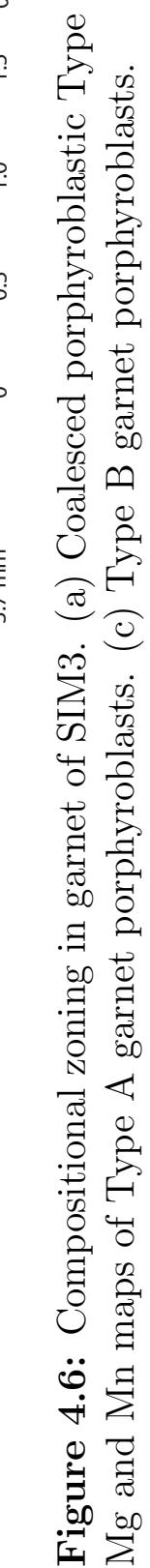



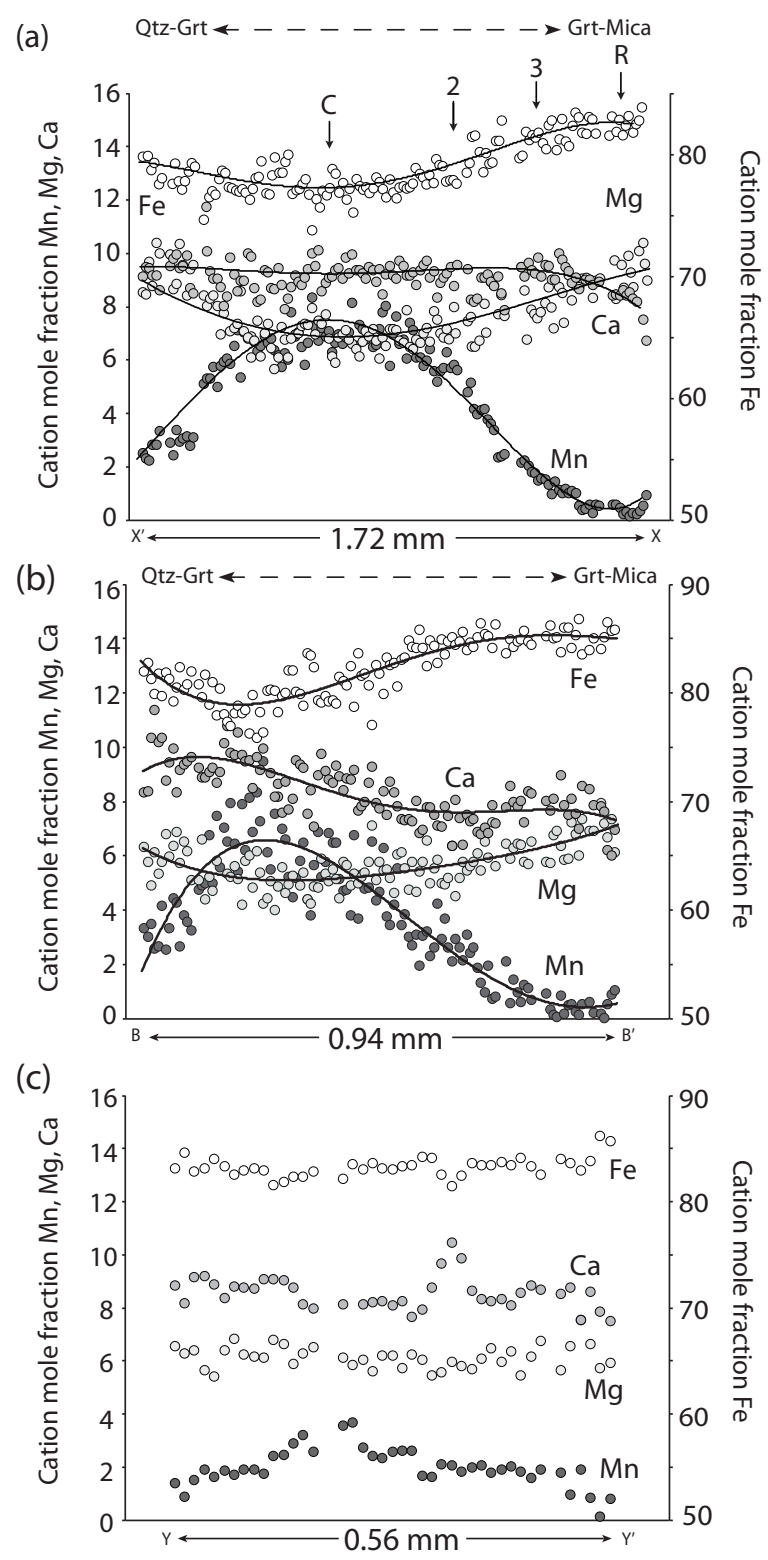

Figure 4.7: Major element compositional profiles of garnet from quantified EMPA maps in Fig. 4.6. (a) and (b) Profiles across Type A garnet. (c) Profile across Type B garnet. Profiles correspond to transects marked on Fig. 4.6 a and c. 

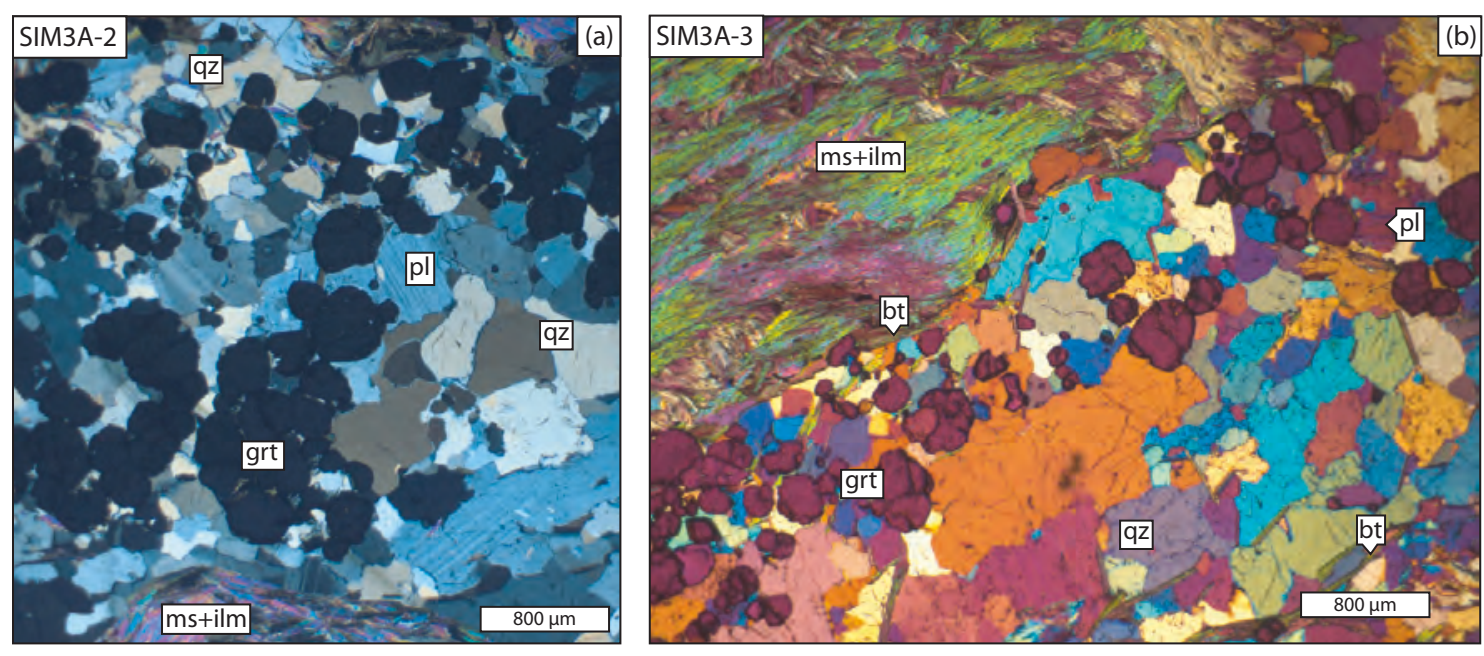

Figure 4.8: (a) Cross-polarised photomicrograph of Type 2 garnet in SIM3. (b) Cross-polarised photomicrograph with quartz plate of Type 2 garnet in SIM3. 
(a)

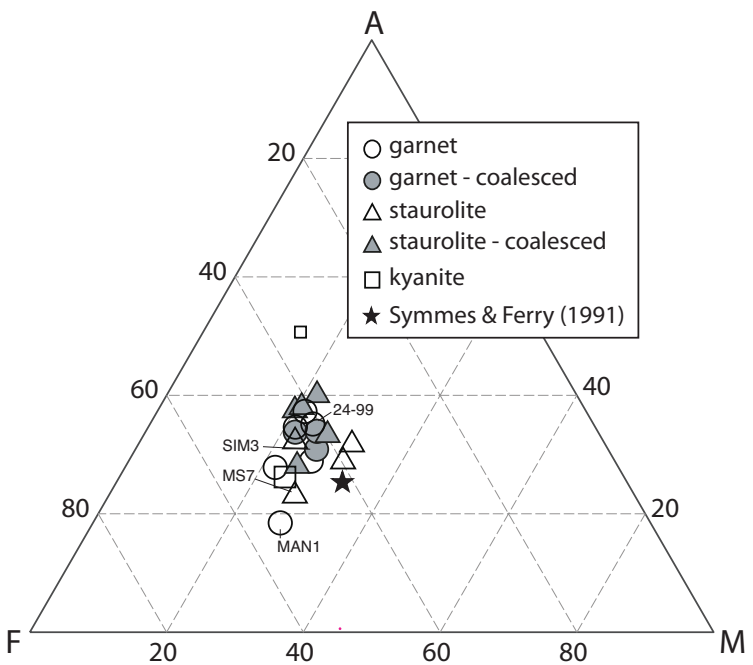

(c)

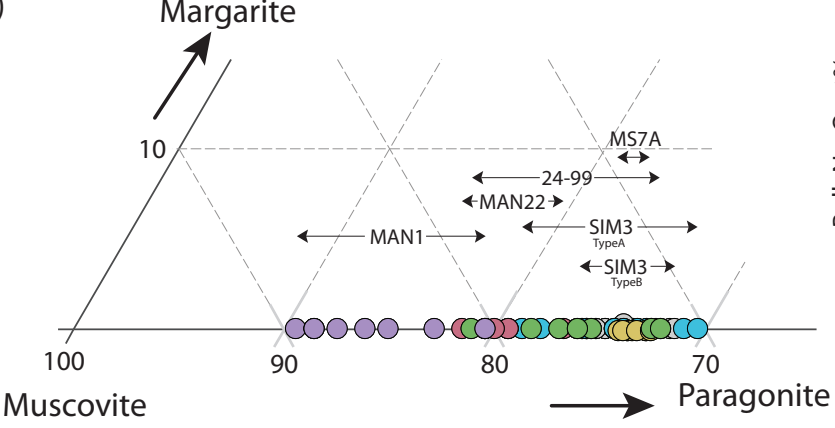

(b)
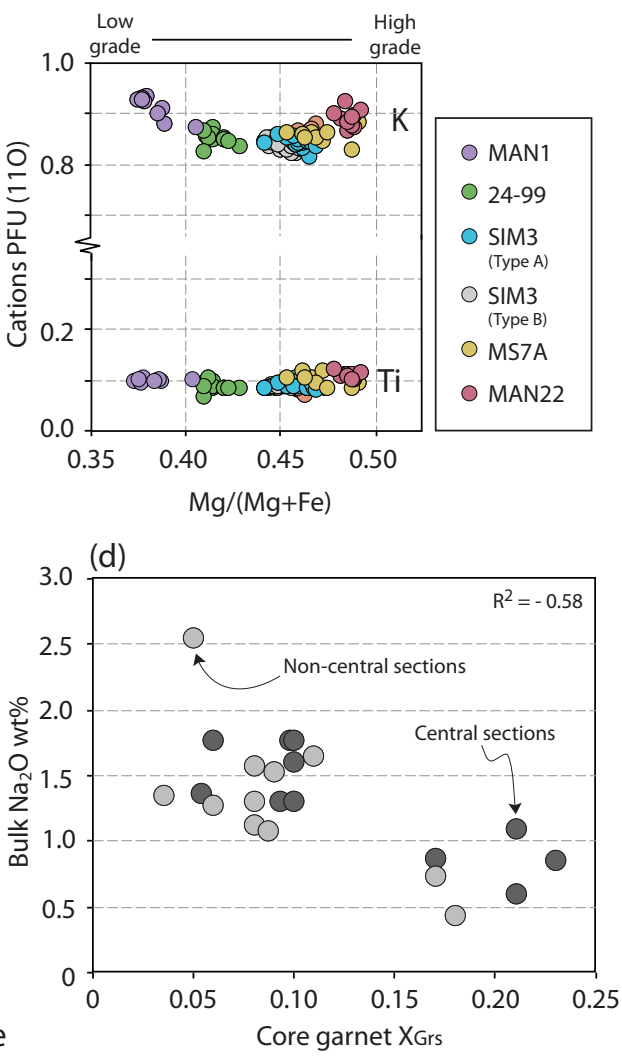

Figure 4.9: (a) AFM projection (Thompson, 1957) from muscovite, quartz and $\mathrm{H}_{2} \mathrm{O}$ of the whole-rock chemistries of samples along the northern transect of the inverted Barrovian Sequence in Sikkim. All iron is $\mathrm{FeO}$ and the average pelite of Symmes \& Ferry (1991) is highlighted. Sample symbols coded according to grade and porphyroblastic garnet texture (Filled symbols represent samples containing coalesced post-kinematic garnet, unfilled represent pre-to-syn kinematic garnet populations). Samples discussed explicitly in this study (Table 3) are labelled. (b) Biotite $\mathrm{K}$ and Ti per formula unit (calculated for 11 oxygens) and $\mathrm{Mg} /\left(\mathrm{Mg}+\mathrm{Fe}^{2+}\right)$. (c) Partial $\mathrm{Ca}-\mathrm{K}-\mathrm{Na}$ ternary diagram for white mica. (d) Maximum grossular in garnet and bulk $\mathrm{Na}_{2} \mathrm{O}$. Dark circles are $\mathrm{X}_{\text {grs }}$ obtained from geometrically central sections, paler circles are $\mathrm{X}_{\text {grs }}$ obtained from random sections. 

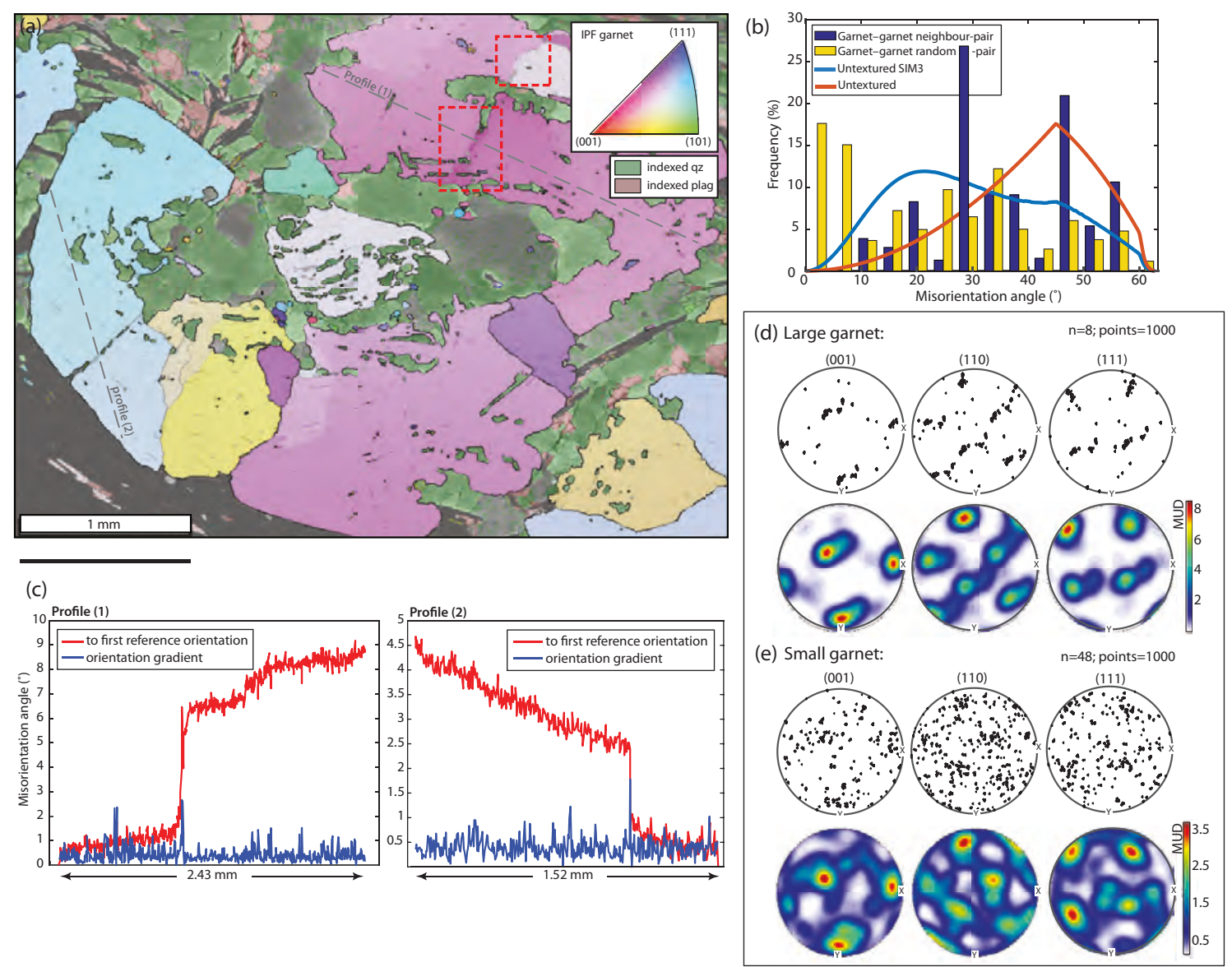

Figure 4.10: EBSD data for SIM3 Map 1 (containing Type A garnet). (a) Euler orientation map of garnet cluster overlain on pattern quality map (PQM). Grain boundaries marked in black, subgrain boundaries in grey. Successfully indexed quartz and plagioclase highlighted in PQM in green and red, respectively. (b) Histogram of angle distribution of garnet-garnet neighbour-pair grain boundaries (blue) and random-pair boundaries. Uncorrelated angle distribution of the SIM3 garnet orientation distribution function (ODF) and a random ODF for garnet plotted in blue and red, respectively. (c) Internal misorientations within garnet grains, along profiles in (a). Red line is misorientation relative to first point in the profile, and blue is the misorientation gradient along the line. (d) Pole figures and corresponding ODF for 8 large garnets in cluster. (e) As in (d) for the 48 small garnets in Map 1 region. 

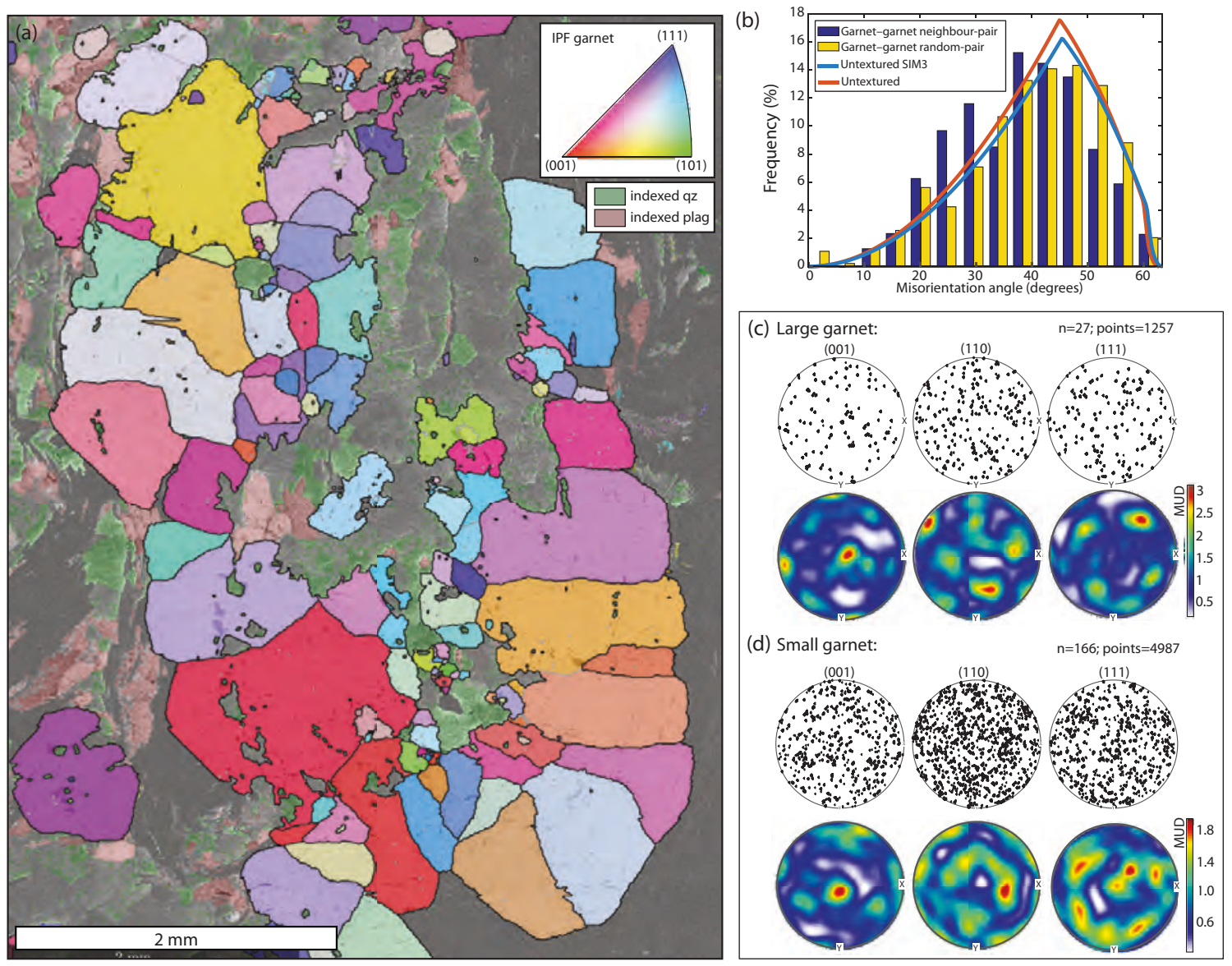

Figure 4.11: EBSD data for SIM3 Map 2 (containing Type A garnet). (a) Euler orientation map of garnet cluster overlain on pattern quality map (PQM). Grain boundaries marked in black, subgrain boundaries in grey. Successfully indexed quartz and plagioclase highlighted in PQM in green and red, respectively. (b) Histogram of angle distribution of garnet-garnet neighbour-pair grain boundaries (blue) and random-pair boundaries. Uncorrelated angle distribution of the SIM3 garnet orientation distribution function (ODF) and a random ODF for garnet plotted in blue and red, respectively. (c) Pole figures and corresponding ODF for 27 large (mainly marginal) garnets in cluster. (d) As in (c) for the 166 small garnets in Map 2 region. 
(a)

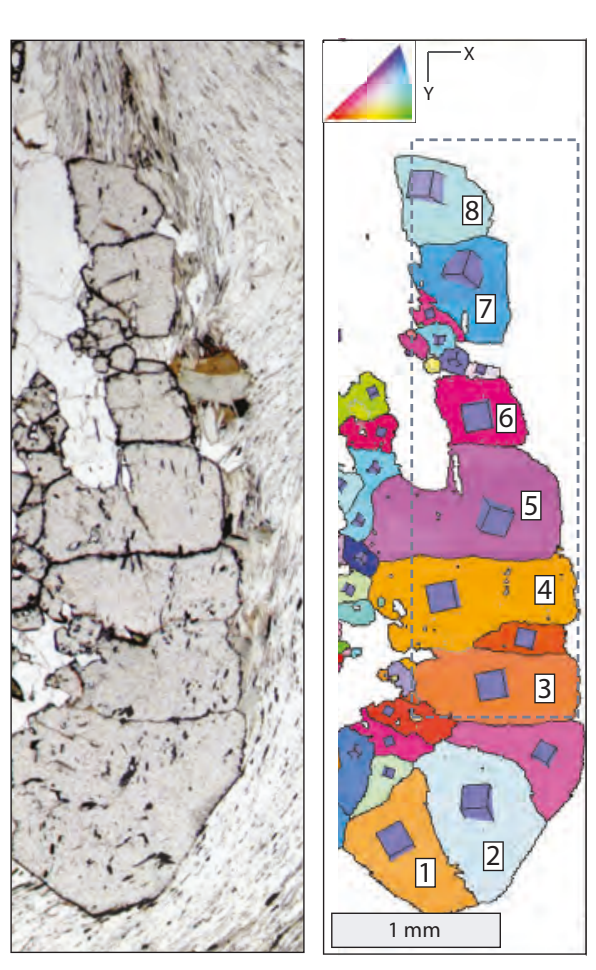

(c)
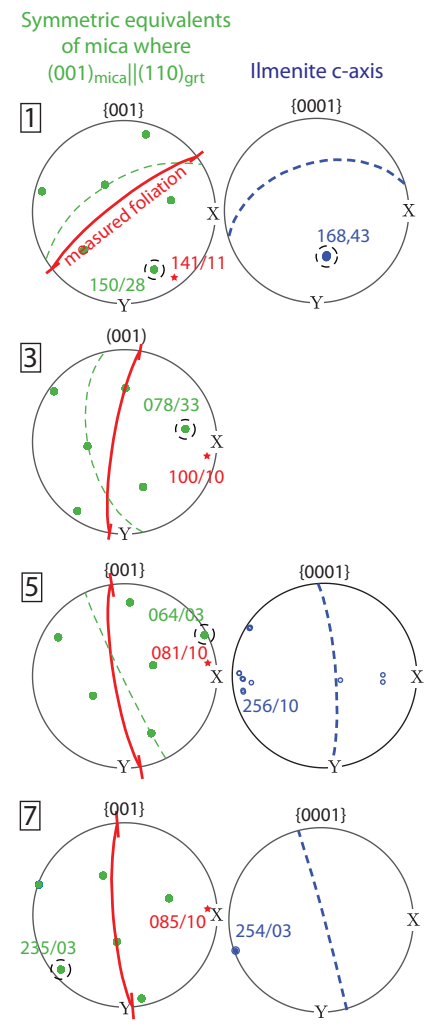
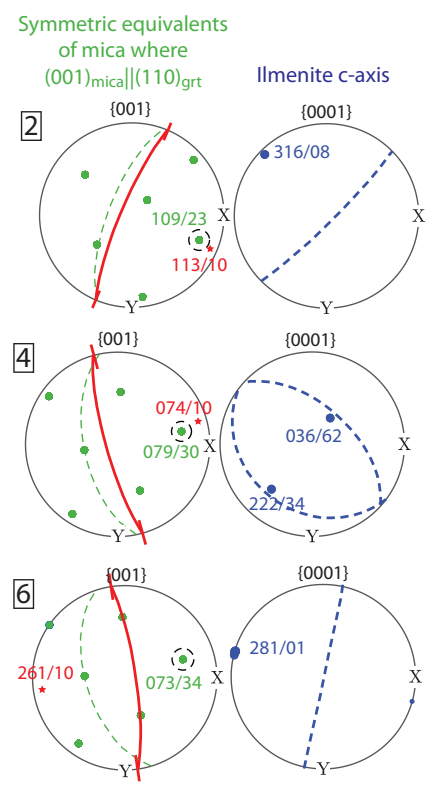

8

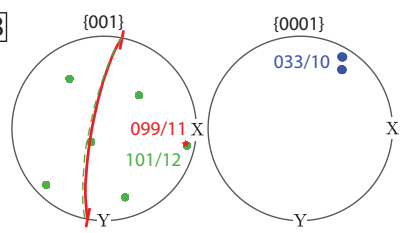

Figure 4.12: (a) Plane-polarised photomic rograph of region mapped with EBSD (Map 1). (b) Euler EBSD map with $\{001\}$ cubic orientations plotted. (c) Measured garnet and simulated epitaxial mica (green), and measured ilmenite (blue) pole figures for corresponding grains in (b). Estimated internal foliation in red, and pole to foliation plotted (star). Poles most likely to correspond to orientation of included ilmenite are circled. 


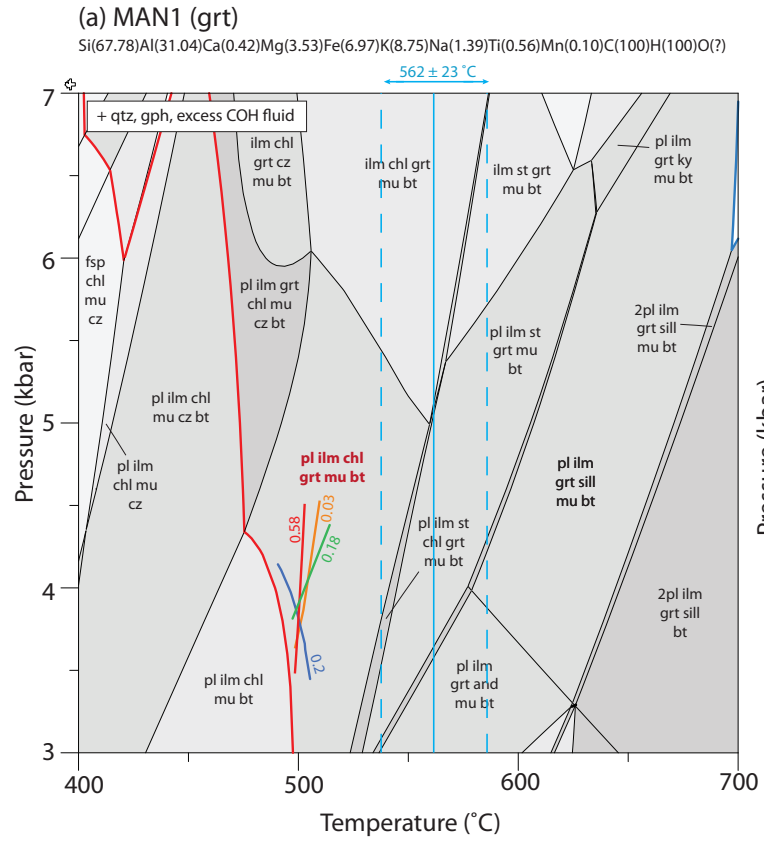

(b) MS7A (st)

$\mathrm{Si}(61.68) \mathrm{Al}(37.53) \mathrm{Ca}(0.65) \mathrm{Mg}(4.07) \mathrm{Fe}(7.43) \mathrm{K}(10.20) \mathrm{Na}(2.75) \mathrm{Ti}(0.76) \mathrm{Mn}(0.13) \mathrm{C}(100) \mathrm{H}(100) \mathrm{O}(?)$

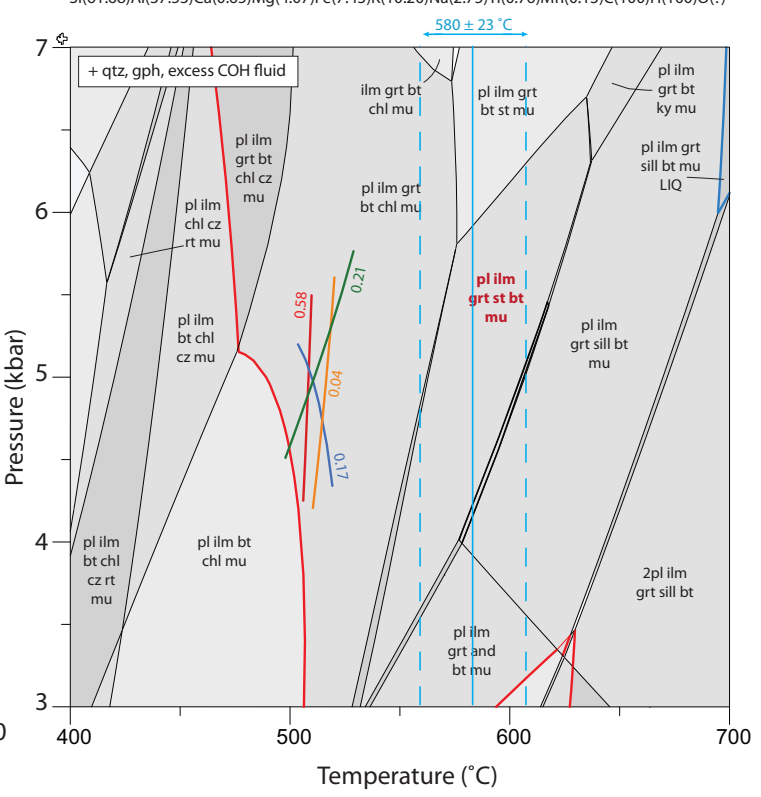

(c) MAN22 (ky)

$\mathrm{Si}(61.31) \mathrm{Al}(29.32) \mathrm{Ca}(0.61) \mathrm{Mg}(5.93) \mathrm{Fe}(11.87) \mathrm{K}(7.53) \mathrm{Na}(1.13) \mathrm{Ti}(0.83) \mathrm{Mn}(0.11) \mathrm{H}(100) \mathrm{C}(100) \mathrm{O}(?)$
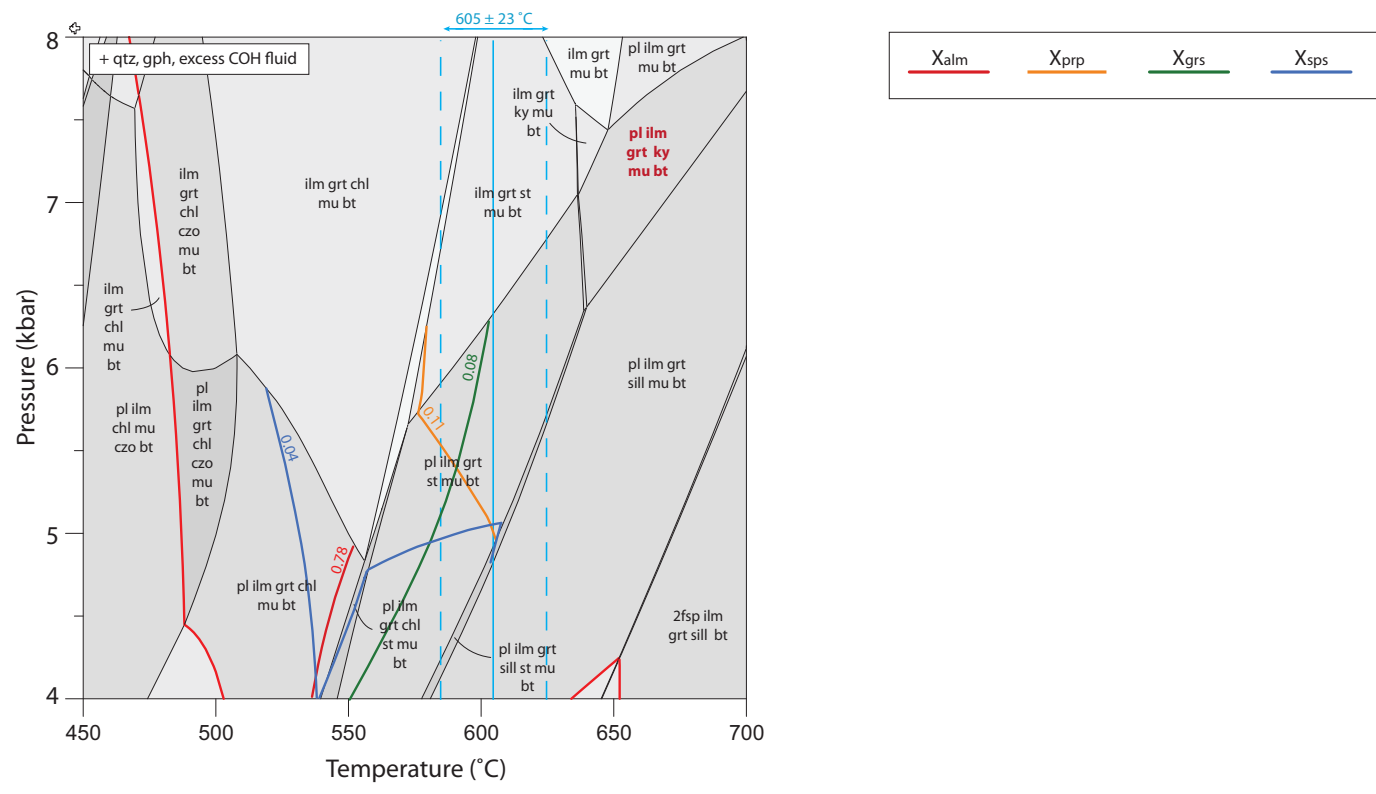

Figure 4.13: Phase equilibria for representative samples with isolated garnet porphyroblasts along northern Sikkim transect. (a) Garnet zone samples MAN1.

(b) Staurolite zone sample MS7A. (c) Kyanite zone sample MAN22. Assemblages in relevant fields labelled (observed mineral assemblage highlighted in red). Average result of Henry et al. (2005) Ti-in-biotite thermometer calculation for each sample overlain on each plot. Core isopleth intersections determined from (Fig. 4.4) are plotted. All models have excess quartz, graphite and a $\mathrm{COH}$ fluid. 
(a) MAN1

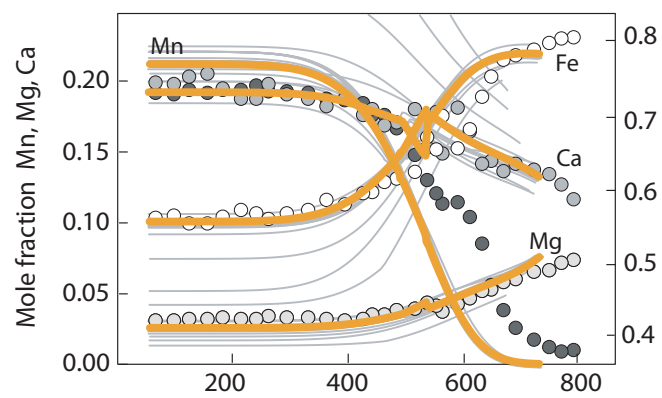

(b) MS7A

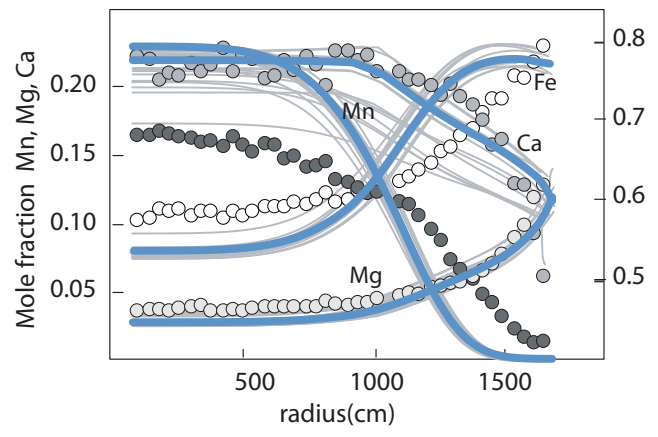

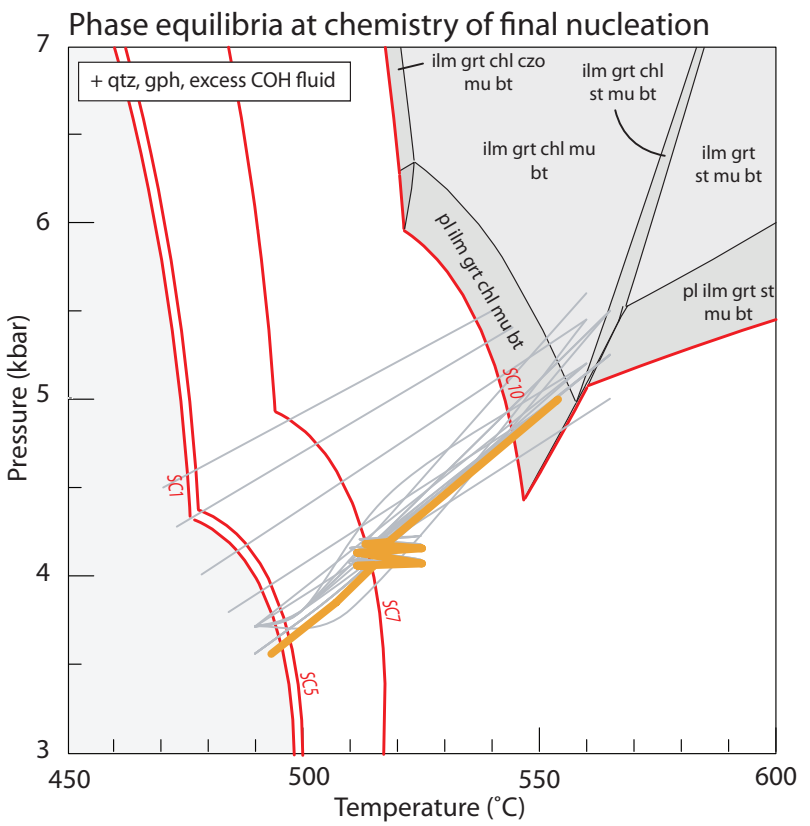

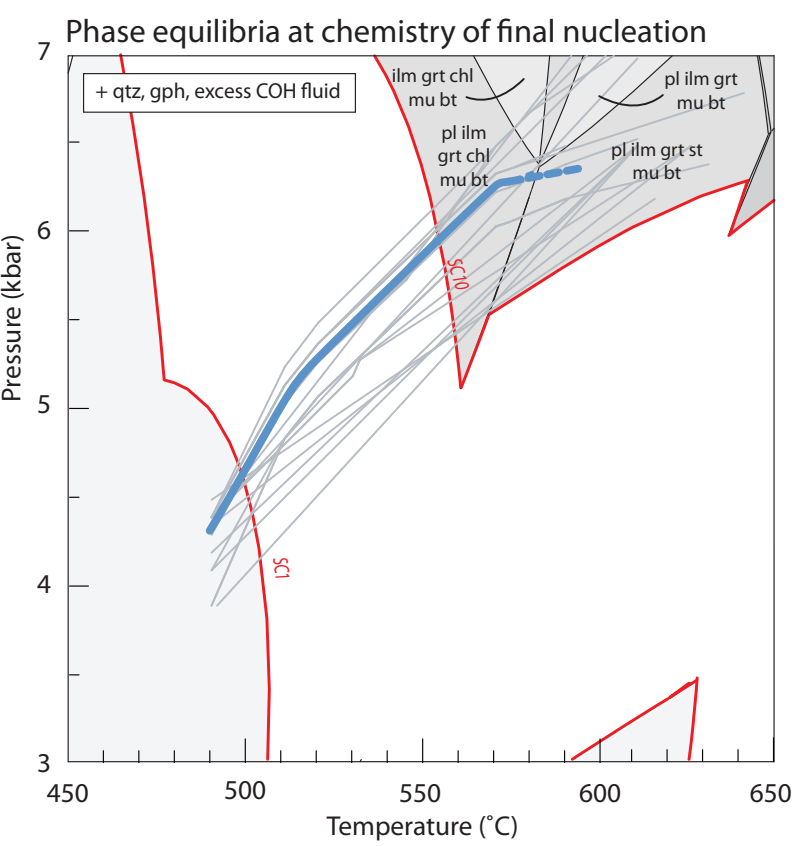

Figure 4.14: THERIA_G forward modelling of central sections. Best fit core-rim modelled profiles along $P-T$ for (a) MAN1 and (b) MS7A. Final phase equilibria calculated using fractionated bulk corresponding to the chemistry at nucleation of the final CSD class of garnet (SC10). 
(a) SIM3 Type A bulk

core $=\mathrm{Si}(74.85) \mathrm{Al}(23.27) \mathrm{Ca}(0.60) \mathrm{Mg}(2.08) \mathrm{Fe}(5.51) \mathrm{K}(5.40) \mathrm{Na}(2.93) \mathrm{Ti}(0.83) \mathrm{Mn}(0.06) \mathrm{C}(100) \mathrm{H}(100) \mathrm{O}($ ? $)$

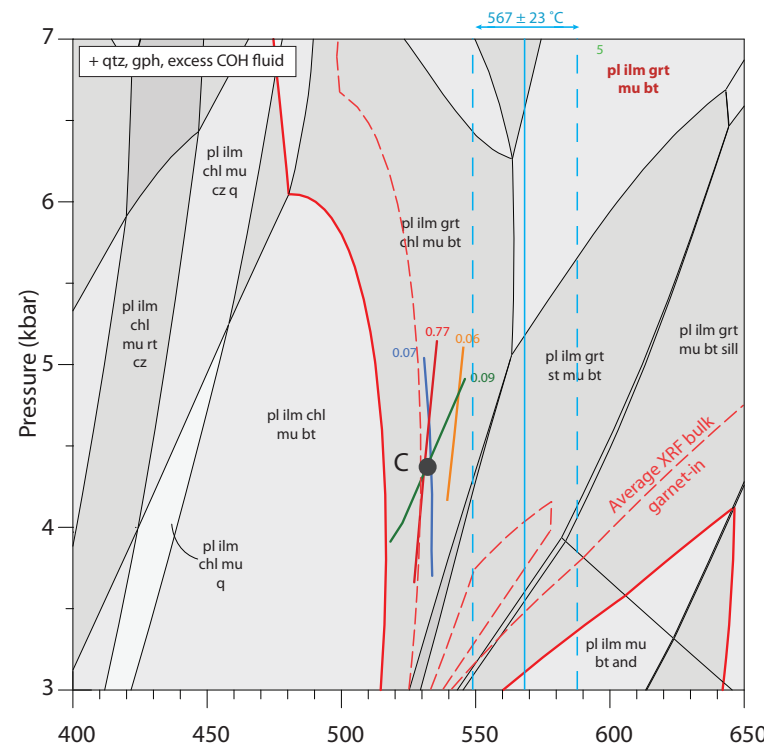

(c) SIM3 Type A fractionated

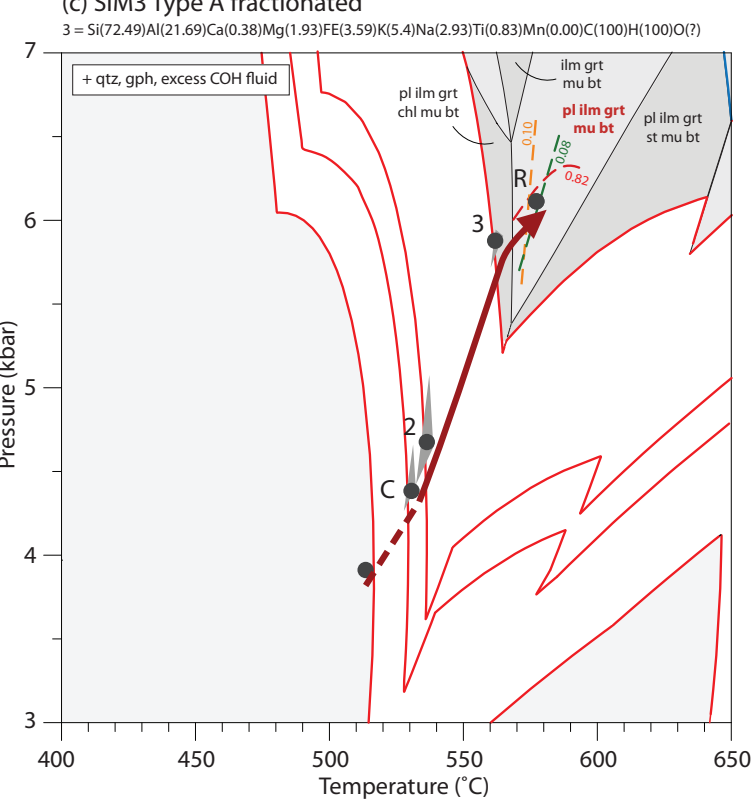

(b) SIM3 Type B bulk

$\mathrm{Si}(74.61) \mathrm{Al}(23.68) \mathrm{Ca}(0.67) \mathrm{Mg}(2.43) \mathrm{Fe}(4.86) \mathrm{K}(5.49) \mathrm{Na}(3.47) \mathrm{Ti}(0.88) \mathrm{Mn}(0.03) \mathrm{C}(100) \mathrm{H}(100) \mathrm{O}(?)$

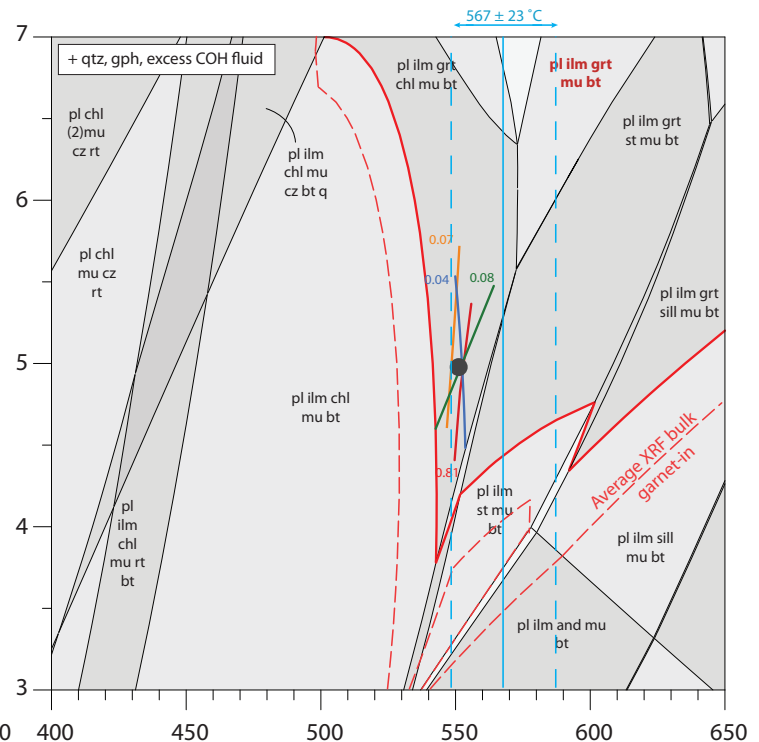

(d) SIM3 Type B fractionated

$\mathrm{R}=\mathrm{Si}(72.78) \mathrm{Al}(22.46) \mathrm{Ca}(0.52) \mathrm{Mg}(2.27) \mathrm{Fe}(3.37) \mathrm{K}(5.49) \mathrm{Na}(3.47) \mathrm{Ti}(0.88) \mathrm{Mn}(0.00) \mathrm{C}(100) \mathrm{H}(100) \mathrm{O}(?)$

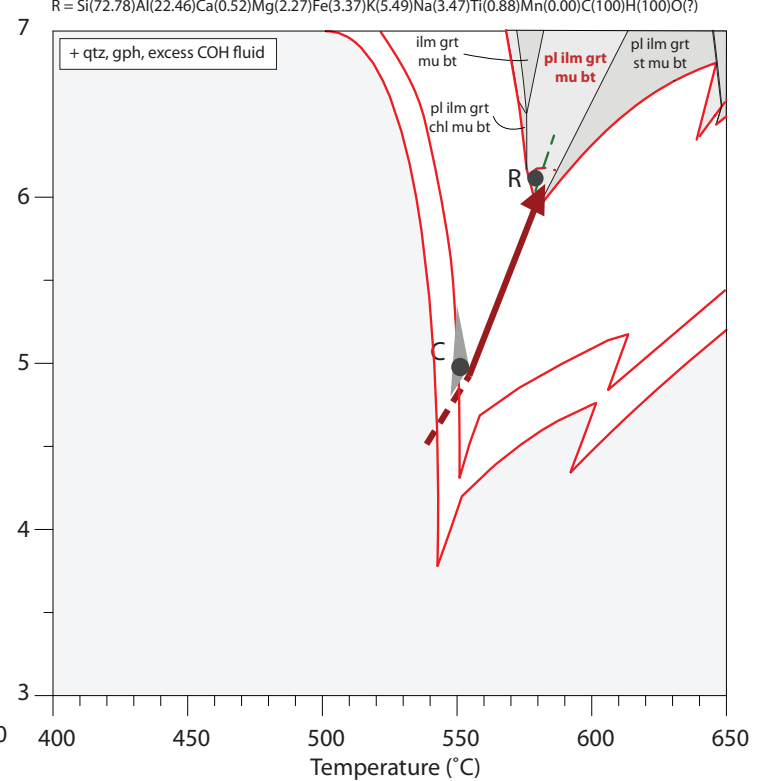

Figure 4.15: Phase equilibria for SIM3 using restricted bulk chemical compositions according to garnet porphyroblast morphology. (a) Type A garnet, (b) Type B garnet. In both (a) and (b), the best estimate of core isopleth intersection for the given bulk composition is plotted. Average result of Henry et al. (2005) Ti-in-biotite thermometer calculation for each sample overlain. (c) and (d) $P-T$ paths for (a) and (b), respectively, determined by fractionating garnet chemical composition from bulk. Evolving garnet-in and phase equilibria recalculated using modified bulks. Measured rim isopleths in fractionated system are shown, and observed assemblage field highlighted. 

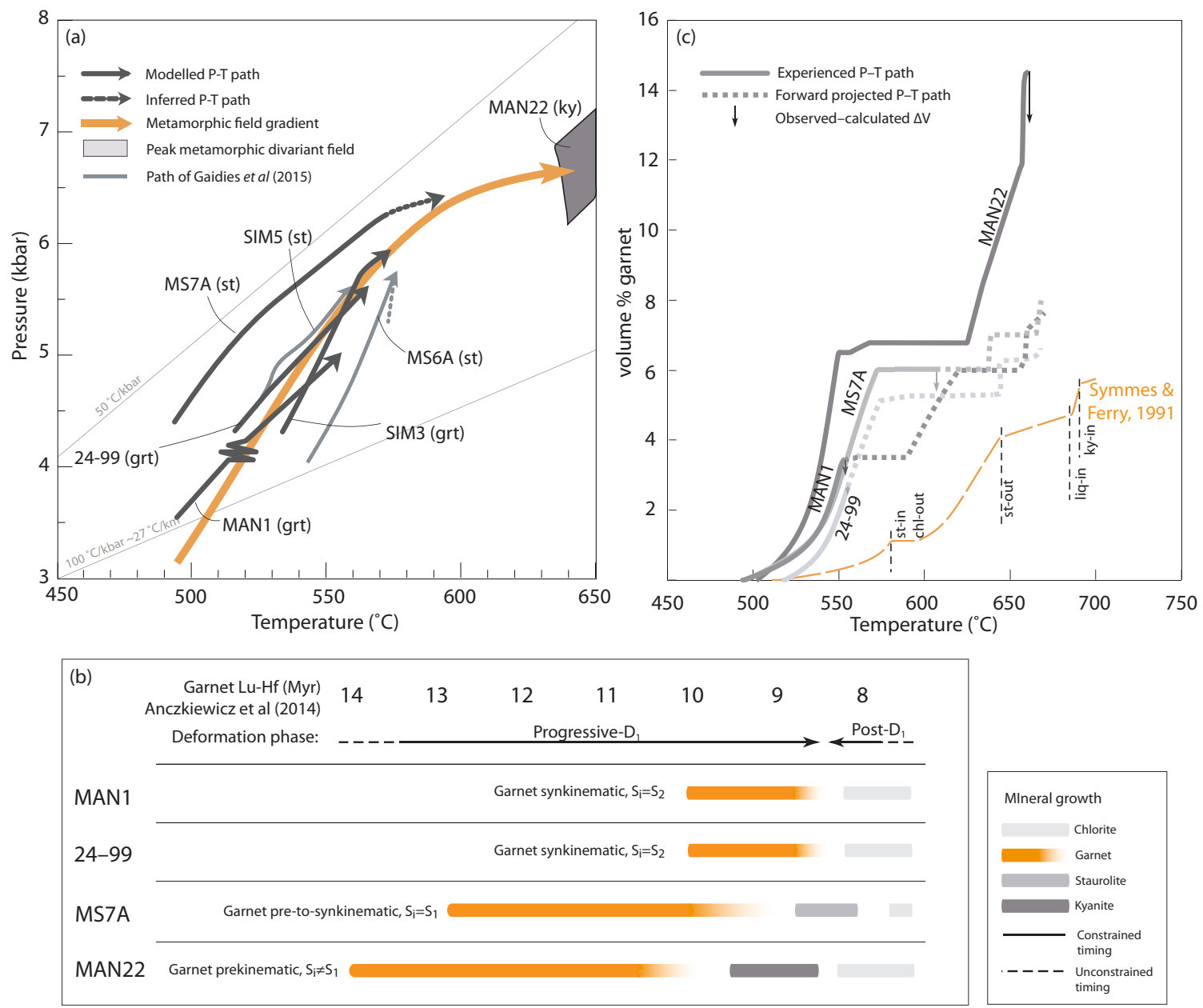

Figure 4.16: (a) Combined $P-T$ results of this study and select results from Gaidies et al. (2015). Paths are taken from Figs. 4.14 and 4.15, and MAN22 field from Fig. 4.13. (b) Summary of garnet growth and microstructure development in samples from this study relative to deformation and $\mathrm{Lu}-\mathrm{Hf}$ garnet ages from Anczkiewicz et al. (2014). (c) Predicted volume percent evolution of garnet along fractionating $P-T$ paths in (a). $P-T$ path for Symmes \& Ferry follows same trajectory as field gradient in (a) to slightly higher $T$ s. 

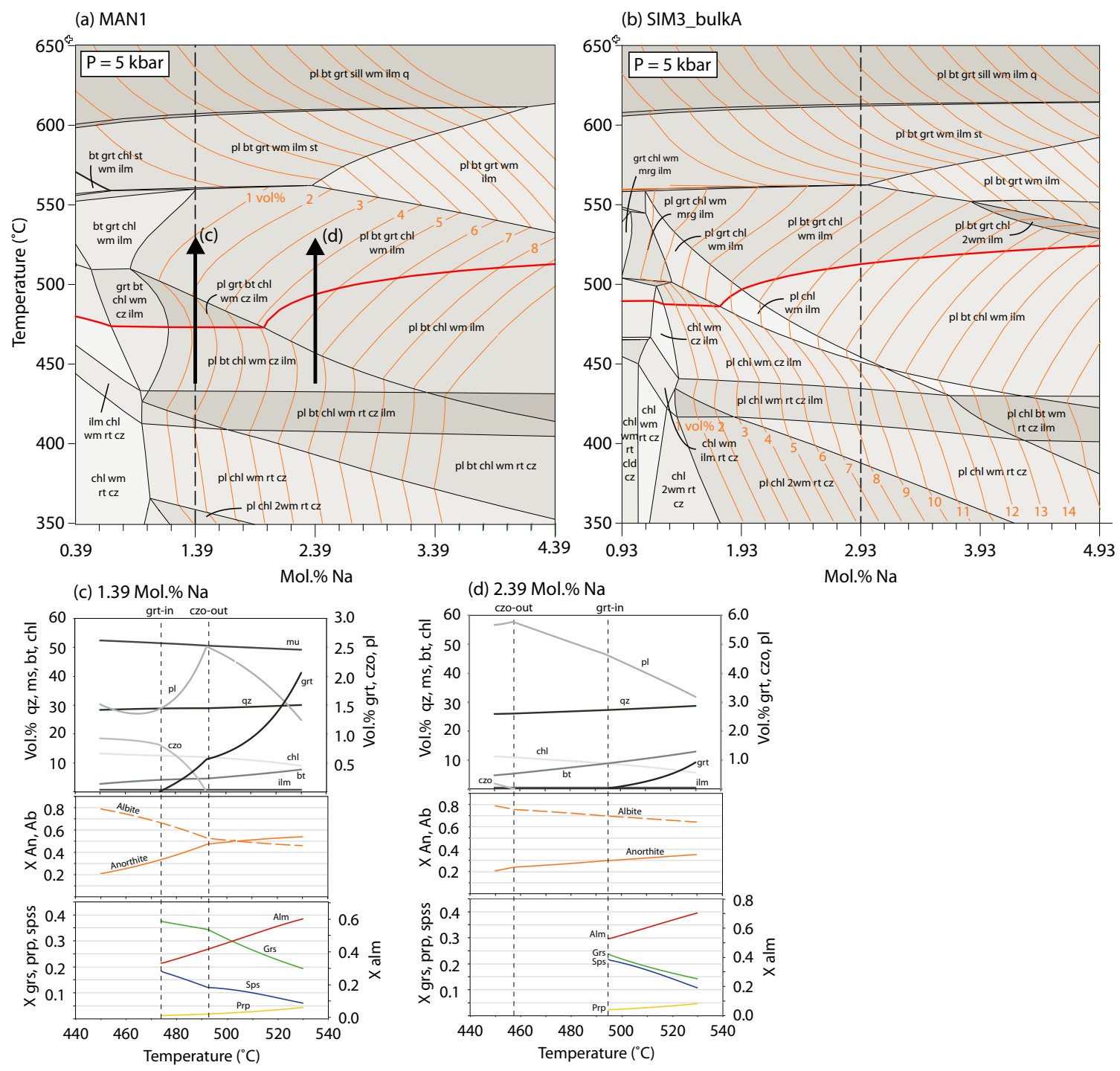

Figure 4.17: Pseudobinary for mole $\mathrm{Na}-T\left(^{\circ}\right)$ at $5 \mathrm{kbar}$ for (a) the MAN1 XRFderived chemistry, and (b) the restricted chemistry determined for the coarse fraction of SIM3. In both figures, the actual bulk $\mathrm{Na}$ is denoted by a dashed line. Fields shaded to denote variance, and garnet-in univariant curve highlighted in red. (c) and (d) Modal mineralogy and plagioclase and garnet chemistry along isobaric $P-T$ path at $1.39 \mathrm{~mol} \% \mathrm{Na}$ bulk, and $2.39 \mathrm{~mol} \% \mathrm{Na}$ bulk, respectively. 


\section{Chapter 5}

\section{Summary and Conclusions}

This thesis builds upon a long tradition of using the compositional zoning contained within garnet porphyroblasts to constrain the nature of and controls on nucleation and growth in metamorphic rocks. Made possible by 3D tomographic methods and rapid analytical approaches, it has produced one of the most extensive chemical and microstructural characterisations of its kind, and has developed a comprehensive view of the rock system at the $P$ s and $T$ s of nucleation and growth of the garnet populations studied. As has been discussed, the widely held assumption that reactions in regionally metamorphosed rocks take place under conditions that closely approximate chemical equilibrium is, perhaps justifiably, under increasing scrutiny in the field of modern metamorphic petrology. However, results of this thesis go to further highlight the efficacy of the equilibrium model of rock metamorphism, and suggest that disequilibrium should not be considered ubiquitous in all rocks but should always be evaluated critically in each separate circumstance.

Below, a summary of some of the key conclusions from this thesis is presented.

In Chapter 2, numerical simulation of garnet nucleation and growth using a chemically fractionating succession of equilibrium states reproduces both the measured 
crystal size distribution and the major element chemical zoning of the entire garnet population. Continuous nucleation of garnet is predicted between incipient garnet crystallisation at c. $520{ }^{\circ} \mathrm{C} / 4.5 \mathrm{kbar}$ and peak metamorphic conditions at c. 565 ${ }^{\circ} \mathrm{C} / 5.6$ kbar, consistent with $P-T$ paths widely accepted for the LHS of the Sikkim Himalaya. Simulation of multicomponent intracrystalline diffusion of the major element chemical composition within the garnet population indicates that rates of metamorphic heating in Sikkim exceeded $100{ }^{\circ} \mathrm{C} \mathrm{Myr}^{-1}$, yielding correspondingly rapid radial growth rates on the order of $1.4 \mathrm{~mm} \mathrm{Myr}^{-1}$. The rapid rates of metamorphism inferred would usually necessitate metamorphism associated with a series of thrust slices, at odds with thermobarometric and microstructural evidence in this and other studies for metamorphism of the Barrovian sequence as an almost entirely coherent block. This study therefore contributes to an increasing body of literature that indicates the need for reassessment of thermal models of regional metamorphism.

Given the short timescales over which crystallisation is modelled to have occurred (on the order of $1 \mathrm{Myr}$ ), the ability to reproduce both the chemistries and the CSD of the garnet population using equilibrium assumptions and an interface-controlled volume distribution in Chapter 2 is unexpected, as it indicates that the departure from equilibrium may not have been significant at any point in the crystallisation history, even in these rapidly heated rocks. Rates of transport in the matrix were exceedingly fast relative to $P-T-t$ changes across the volume, and interfacial-processes are concluded to be of critical importance to controlling the rate of mineral crystallisation. Subtle variations in the major element zoning from large to small garnets may indicate that garnet growth was more strictly controlled by a size-dependent interface-controlled rate law, further evidence for the importance of the interface in controlling crystallisation.

By developing the dataset in Chapter 2 further, Chapter 3 shows that lengthscales 
of equilibration of a range of trace elements are often decoupled, both from each other and from major elements at given $P-T-t$ conditions, evinced with the first comprehensive dataset of trace element zoning within a whole population of garnets using a LA-ICP-MS 2D mapping approach. More than anything, assessment of trace elements in garnet has highlighted their potential for future interpretations of crystallisation histories, and how little we still know about the controls on the distribution of trace elements in both garnet and the matrix and whether or not they systematically record processes operating during crystallisation.

There is evidence for a spatially and temporally heterogeneous HREE+Y distribution in the rock matrix over lengthscales of less than centimetres, with strongly diffusion-limited incorporation of these elements into garnet. Enrichment of HREE+Y in the cores of garnet of all sizes is at odds with their characteristic disequilibrium across the matrix. These variations may imply subtle variations in the controls on crystallisation that would otherwise go unnoticed with major element analysis alone, namely the potential for nucleation to be favourably influenced by HREEs. Asymmetric oscillatory annular zoning in Chapter 3 indicates that radial growth rates determined in Chapter 2 may have fluctuated more than indicated by diffusion modelling, at a scale not resolvable in major element zoning. Smooth zoning of many transition metals indicate that their distribution in garnet may be controlled by equilibrium partitioning between garnet and the rock matrix, and LA-ICP-MS reveal Li coupling to HREE. REEs and Y document increases in the lengthscale of equilibration across garnet surfaces during the $P-T$ interval of crystallisation, and others $(\mathrm{Cr})$ are fully immobile throughout the duration of crystallisation in both the matrix and along garnet interfaces. Those elements that maintain disequilibrium attest to aspects of the continuous deformation history of the population, with calculated strain rates on the order of $10^{-11}$ to $10^{-12} \mathrm{~s}^{-1}$. This zoning may indirectly contribute to the 
long-standing debate surrounding the nature of relative rotation versus non-rotation of porphyroblasts and/or the matrix during growth.

Most metapelites from Sikkim exhibit populations seemingly analogous to that presented in Chapter 2, and broadly exhibit systematic variations in grain size and abundance according to their position along the metamorphic field gradient. Thermobarometric constraints in Chapter 4 indicate that this correlation cannot be related to systematic differences in the critical $T$ for nucleation, but may instead be consistent with porphyroblast ripening from low-to-high grades, $P-T$ path length, and variations inherently derived from differences in bulk chemical composition. Exposure of a unit containing deviations from this trend is related to late slice tectonics along the proposed Mangan-Sankhalan Thrust that emplaced an out-of-sequence section of the Daling group that was thermally or kinematically decoupled from adjacent portions of the IMS, but a part of the same nested $P-T$ history.

Chapter 4 also illustrates the effect that a highly segregated matrix may have on both the attainment of localised chemical equilibrium during garnet crystallisation and the development of garnet morphology. For the case in which the matrix microstructure was strongly segregated during rapid rates of heating and metamorphism, the morphology and chemistry of garnet developed during its growth was not exclusively limited by crystallographic constraints or equilibrium partitioning, but by the relative availability of components at any given surface (i.e., transport-limited growth). The microstructures in Chapter 4, compared with others presented in the study, therefore highlight the importance of grain boundaries in controlling effective matrix diffusivities, which in turn facilitate morphology development. However, it should be noted that while chemistries and textures are not equilibrated at a local scale, the equilibrium model still reproduces garnet volumes almost perfectly. This points to the efficacy of the method, despite the influence of kinetics. 
Samples that experienced pre-to-syn kinematic garnet growth in Chapters 2, 3 and 4 may not reliably document a record of the primary sites of garnet nucleation. However, post-kinematic garnet microstructures in Chapter 4 point to a heterogeneous interplay of the variables controlling clustered nucleation on heterogeneously distributed nucleation sites. EBSD indicates that epitaxial crystallisation of garnet on precursor micas facilitated nucleation in domains with appropriate chemical compositions, but was not the principal control on nucleation sites.

\subsection{Potential avenues of future work}

From a general perspective, application of a comprehensive suite of similar methods to those presented in this thesis to samples from both similar and distinct tectonic settings will better enable us to understand to what extent results from Sikkim represent 'the exception,' or 'the rule' in metamorphic petrology. It may be the case that the rapid rates of metamorphism experienced by rocks in Sikkim make the region almost uniquely placed to elucidate principal controls on regional rock metamorphism, and systematic evaluation of the same parameters in slowly heated terranes will elucidate ubiquitous key processes.

Continued investigation of microstructures, major and trace element chemistries, and the relative ages of metamorphic minerals in a wide range of metamorphic terranes is required to improve our understanding of how these characteristics record the history of solid-state mineral crystallisation. Although this thesis has contributed to an expanding body of work on these topics, broad uncertainties still surround whether

textures can be used to systematically infer the extent of equilibration, the role of deformation in facilitating mineral crystallisation, and whether or not trace elements systematically retain a record of processes acting at high- $T$ and high- $P$. In particular, 
developing our empirical understanding of controls on heterogeneous distribution of nucleation sites may facilitate the development of better quantitative models to link deformation and porphyroblast crystallisation. This may yield a better understanding of 3D texture formation and would be an exciting avenue of research with broad relevance to the metamorphic community.

The results of this work have also highlighted more specific future research directions for the samples from this study, several of which are being enacted currently and all of which would serve to develop the work in this thesis. Several key suggestions are presented below.

- The strain rates presented in Chapter 3 make the assumption of homogeneous bulk shear of single spherical objects, with no boundary conditions, interacting bodies or systemic heterogeneities. In reality, and as discussed in Chapter 3, interactions between imposed stress and rock texture may yield differential and position-dependent strain rates. Therefore, numerical simulation of the system utilising XR- $\mu \mathrm{CT}$, chemical and thermobarometric constraints and a coupled metamorphism-deformation numerical model would enable a more comprehensive assessment of the development of immobile element spiral zoning and potential degree of garnet-matrix interface coupling, and will test the results in this thesis. The microdynamic software ELLE (Jessell et al., 2001) would be invaluable for this approach. Moreover, this might be of particular use to further investigate the correlation between porphyroblast size, crystal density and grade in Barrovian sequences, as the multi-process simulation allows simulation of the simultaneous processes of nucleation, diffusion, growth and deformation.

- The potentially transport-limited heterogeneous porphyroblastic microstructures presented in Chapter 4 exhibit evidence for having developed as a conse- 
quence of channelised grain boundary diffusion through quartzo-feldspathic domains. Little is known about the chemical and structural nature of grain boundaries in polyphase metamorphic rocks, and the potential presence of diffusional gradients surrounding porphyroblasts has previously only been documented on the basis of assemblage haloes around growing porphyroblasts (e.g., Miyazaki, 2015). There have as yet been no direct observations of diffusional gradients or disequilibrium preserved in grain boundary pathways, although there has been some attempts at constraining the chemistry of the grain boundary (e.g., Keller et al., 2008). Documentation of the presence of such chemical gradients would open the doors to better estimation of grain boundary diffusivities and the rate of propagation of diffusion haloes. Evidence for grain boundary transfer is therefore currently being sought using preliminary FE-EPMA mapping of grain boundaries propagating away from protrusions of garnet, and depending on results of this analysis, atom probe work will enable better investigation of the grain boundary at the nm-scale. Preliminary FE-EPMA results (Fig. 5.1) suggest there may be a heterogeneous distribution of elements present along grain boundaries, and most interestingly, Al can be seen to decrease in abundance towards the garnet surface in Fig. 5.17.

- An additional LA-ICP-MS trace element study of the polycrystalline garnets presented in Chapter 4 would help to confirm the potential control of HREE on distribution of nucleation sites of garnet, as was proposed in Chapter 3.

- High-resolution garnet geochronology using microdrilling Lu-Hf and Sm-Nd approaches would test many of the proposed timings in this study. Spatially resolved geochronology was originally intended for Chapter 1 to compare relative ages of growth domains to the constant volumetric growth rate assumption, but 
garnets were unsuitable. Absolute geochronological constraints on the irregular garnet populations presented in Chapter 4 would be valuable in order to test the suggestions of relative timing of garnet crystallisation.
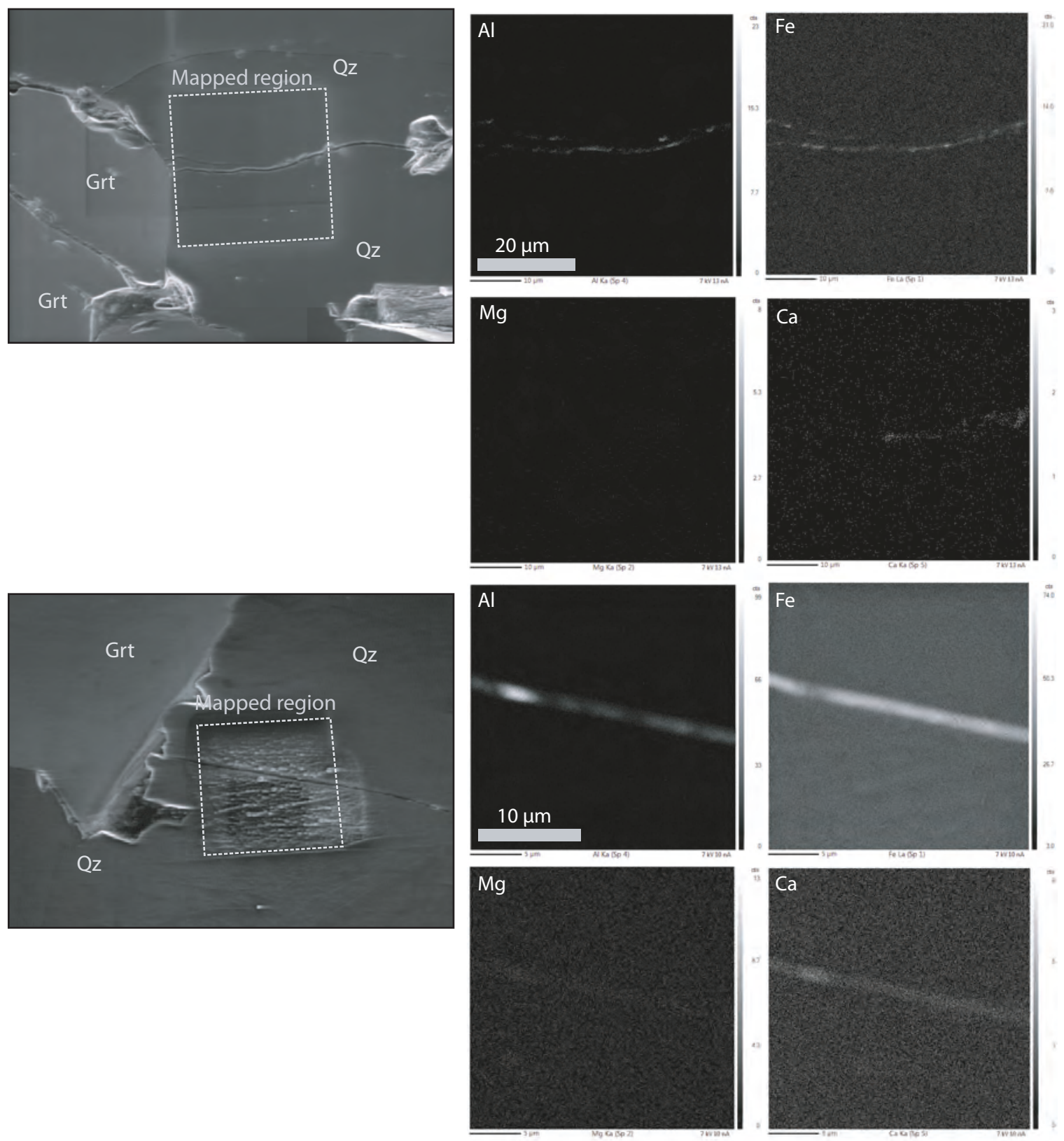

Figure 5.1: Preliminary FE-EPMA maps of quartz-quartz grain boundaries adjacent to garnet-quartz- -quartz triple junctions. 


\section{Bibliography}

Ague, J. J., 1991. Evidence for major mass transfer and volume strain during regional metamorphism of pelites. Geology 19 (8), 855-858.

Ague, J. J., 1994. Mass transfer during Barrovian metamorphism of pelites, southcentral Connecticut; I, Evidence for changes in composition and volume. American Journal of Science 294 (8), 989-1057.

Ague, J. J., Baxter, E. F., 2007. Brief thermal pulses during mountain building recorded by Sr diffusion in apatite and multicomponent diffusion in garnet. Earth and Planetary Science Letters 261 (3), 500-516.

Ague, J. J., Carlson, W. D., 2013. Metamorphism as garnet sees it: The kinetics of nucleation and growth, equilibration, and diffusional relaxation. Elements 9 (6), 439-445.

Anczkiewicz, R., Chakraborty, S., Dasgupta, S., Mukhopadhyay, D., Kołtonik, K., 2014. Timing, duration and inversion of prograde Barrovian metamorphism constrained by high resolution Lu-Hf garnet dating: A case study from the Sikkim Himalaya, NE India. Earth and Planetary Science Letters 407, 70-81.

Anczkiewicz, R., Thirlwall, M., Alard, O., Rogers, N. W., Clark, C., 2012. Diffusional homogenization of light REE in garnet from the Day Nui Con Voi Massif in NVietnam: Implications for Sm-Nd geochronology and timing of metamorphism in the Red River shear zone. Chemical Geology 318, 16-30.

Anderson, D. E., Olimpio, J., 1977. Progressive homogenization of metamorphic garnets, South Morar, Scotland: evidence for volume diffusion. Canadian Mineralogist 15 (2), 205-216.

Ashworth, J., Birdi, J., Emmett, T., 1992. A complex corona between olivine and plagioclase from the Jotun Nappe, Norway, and the diffusion modelling of multimineralic layers. Mineralogical magazine 56 (385), 511-525. 
Atherton, M., Brotherton, M., 1982. Major element composition of the pelites of the Scottish Dalradian. Geological Journal 17 (3), 185-221.

Austrheim, H., 1987. Eclogitization of lower crustal granulites by fluid migration through shear zones. Earth and Planetary Science Letters 81 (2-3), 221-232.

Bachmann, F., Hielscher, R., Schaeben, H., 2010. Texture analysis with MTEX-free and open source software toolbox. In: Solid State Phenomena. Vol. 160. Trans Tech Publ, pp. 63-68.

Baldwin, J., Powell, R., Brown, M., Moraes, R., Fuck, R., 2005. Modelling of mineral equilibria in ultrahigh-temperature metamorphic rocks from the Anápolis-Itauçu Complex, central Brazil. Journal of Metamorphic Geology 23 (7), 511-531.

Balluffi, R. W., Allen, S. M., Carter, W. C., 2005. Kinetics of Materials. John Wiley \& Sons, Inc., Hoboken, New Jersey.

Barr, T. D., Houseman, G. A., 1996. Deformation fields around a fault embedded in a non-linear ductile medium. Geophysical Journal International 125 (2), 473-490.

Baumgartner, J., Dey, A., Bomans, P. H., Le Coadou, C., Fratzl, P., Sommerdijk, N. A., Faivre, D., 2013. Nucleation and growth of magnetite from solution. Nature Materials 12 (4), 310-314.

Bea, F., 1996. Residence of REE, Y, Th and U in granites and crustal protoliths; implications for the chemistry of crustal melts. Journal of petrology 37 (3), 521552.

Bea, F., Montero, P., Garuti, G., Zacharini, F., 1997. Pressure-dependence of rare earth element distribution in amphibolite-and granulite-grade garnets. a LA-ICPMS study. Geostandards and Geoanalytical Research 21 (2), 253-270.

Bea, F., Pereira, M., Stroh, A., 1994. Mineral/leucosome trace-element partitioning in a peraluminous migmatite (a laser ablation-ICP-MS study). Chemical Geology 117 (1-4), 291-312.

Beinlich, A., Klemd, R., John, T., Gao, J., 2010. Trace-element mobilization during ca-metasomatism along a major fluid conduit: Eclogitization of blueschist as a consequence of fluid-rock interaction. Geochimica et Cosmochimica Acta 74 (6), 1892-1922.

Bell, T., 1985. Deformation partitioning and porphyroblast rotation in metamorphic rocks: a radical reinterpretation. Journal of Metamorphic Geology 3 (2), 109-118. 
Bell, T., Johnson, S., 1989. Porphyroblast inclusion trails: the key to orogenesis. Journal of Metamorphic Geology 7 (3), 279-310.

Bell, T., Rubenach, M., Fleming, P., 1986. Porphyroblast nucleation, growth and dissolution in regional metamorphic rocks as a function of deformation partitioning during foliation development. Journal of Metamorphic Geology 4 (1), 37-67.

Berg, C., Carlson, W., Connelly, J., 2013. Strain rates at high temporal resolution from curved inclusion trails in garnet, Passo del Sole, Central Swiss Alps. Journal of Metamorphic Geology 31 (3), 243-262.

Bhattacharyya, K., 2010. Geometry and kinematics of the fold-thrust belt and structural evolution of the major Himalayan fault zones in the Darjeeling-Sikkim Himalaya, India. Ph.D. thesis, University of Rochester.

Bhattacharyya, K., Mitra, G., 2009. A new kinematic evolutionary model for the growth of a duplex - an example from the Rangit duplex, Sikkim Himalaya, India. Gondwana Research 16 (3), 697-715.

Bhattacharyya, K., Mitra, G., 2011. Strain softening along the MCT zone from the Sikkim Himalaya: relative roles of quartz and micas. Journal of Structural Geology 33 (6), 1105-1121.

Biermeier, C., Stüwe, K., 2003. Strain rates from snowball garnet. Journal of Metamorphic Geology 21 (3), 253-268.

Biermeier, C., Stüwe, K., Barr, T., 2001. The rotation rate of cylindrical objects during simple shear. Journal of Structural Geology 23 (5), 765-776.

Bollinger, L., Henry, P., Avouac, J.-P., 2006. Mountain building in the Nepal Himalaya: Thermal and kinematic model. Earth and Planetary Science Letters 244 (12), 58-71.

Bose, P., 1891. Notes on the geology and mineral resources of Sikkim. Rec. Geol. Surv. India 24 (pt 4), 217-230.

Brandon, D., 1966. The structure of high-angle grain boundaries. Acta metallurgica 14 (11), 1479-1484.

Burg, J., Chen, G., 1984. Tectonics and structural zonation of southern Tibet, China. Nature 311 (5983), 219-223.

Burns, R. G., 1993. Mineralogical applications of crystal field theory. Vol. 5. Cambridge University Press. 
Burton, K. W., O'Nions, R. K., 1991. High-resolution garnet chronometry and the rates of metamorphic processes. Earth and Planetary Science Letters 107 (3-4), 649-671.

Burton, W.-K., Cabrera, N., Frank, F., 1951. The growth of crystals and the equilibrium structure of their surfaces. Philosophical Transactions of the Royal Society of London A: Mathematical, Physical and Engineering Sciences 243 (866), 299-358.

Caddick, M. J., Konopásek, J., Thompson, A. B., 2010. Preservation of garnet growth zoning and the duration of prograde metamorphism. Journal of Petrology 51 (11), $2327-2347$.

Cahalan, R. C., Kelly, E. D., Carlson, W. D., 2014. Rates of Li diffusion in garnet: Coupled transport of Li and Y+REEs. American mineralogist 99 (8-9), 1676-1682.

Cahn, J. W., 1957. Nucleation on dislocations. Acta Metallurgica 5 (3), 169-172.

Carlson, W., Gordon, C., 2004. Effects of matrix grain size on the kinetics of intergranular diffusion. Journal of Metamorphic Geology 22 (8), 733-742.

Carlson, W. D., 1989. The significance of intergranular diffusion to the mechanisms and kinetics of porphyroblast crystallization. Contributions to Mineralogy and Petrology 103 (1), 1-24.

Carlson, W. D., 1999. The case against Ostwald ripening of porphyroblasts. The Canadian Mineralogist 37 (2), 403-413.

Carlson, W. D., 2002. Presidential address. Scales of disequilibrium and rates of equilibration during metamorphism. American Mineralogist 87 (2-3), 185-204.

Carlson, W. D., 2006. Dana lecture. rates of Fe, Mg, Mn, and Ca diffusion in garnet. American Mineralogist 91 (1), 1-11.

Carlson, W. D., 2011. Porphyroblast crystallization: linking processes, kinetics, and microstructures. International Geology Review 53 (3-4), 406-445.

Carlson, W. D., 2012. Rates and mechanism of Y, REE, and Cr diffusion in garnet. American Mineralogist 97 (10), 1598-1618.

Carlson, W. D., Denison, C., 1992. Mechanisms of porphyroblast crystallization: results from high-resolution computed X-ray tomography. Science 257 (5074), 12361239. 
Carlson, W. D., Denison, C., Ketcham, R. A., 1995. Controls on the nucleation and growth of porphyroblasts: kinetics from natural textures and numerical models. Geological Journal 30 (3-4), 207-225.

Carlson, W. D., Pattison, D. R., Caddick, M. J., 2015. Beyond the equilibrium paradigm: How consideration of kinetics enhances metamorphic interpretation. American Mineralogist 100 (8-9), 1659-1667.

Carmichael, D. M., 1969. On the mechanism of prograde metamorphic reactions in quartz-bearing pelitic rocks. Contributions to Mineralogy and Petrology 20 (3), $244-267$.

Casey, W. H., Ludwig, C., 1996. The mechanism of dissolution of oxide minerals. Nature 381 (6582), 506.

Cashman, K. V., Ferry, J. M., 1988. Crystal size distribution (CSD) in rocks and the kinetics and dynamics of crystallization. Contributions to Mineralogy and Petrology 99 (4), 401-415.

Castro, A. E., Spear, F. S., 2016. Reaction overstepping and re-evaluation of peak $\mathrm{P}-\mathrm{T}$ conditions of the blueschist unit Sifnos, Greece: implications for the Cyclades subduction zone. International Geology Review, 1-15.

Catlos, E., Dubey, C., Harrison, T., Edwards, M., 2004. Late Miocene movement within the Himalayan Main Central Thrust shear zone, Sikkim, north-east India. Journal of Metamorphic Geology 22 (3), 207-226.

Catlos, E., Harrison, T. M., Kohn, M. J., Grove, M., Ryerson, F., Manning, C. E., Upreti, B., 2001. Geochronologic and thermobarometric constraints on the evolution of the Main Central Thrust, central Nepal Himalaya. Journal of Geophysical Research: Solid Earth 106 (B8), 16177-16204.

Chakraborty, S., Anczkiewicz, R., Gaidies, F., Rubatto, D., Sorcar, N., Faak, K., Mukhopadhyay, D., Dasgupta, S., 2016. A review of thermal history and timescales of tectonometamorphic processes in Sikkim Himalaya (NE India) and implications for rates of metamorphic processes. Journal of Metamorphic Geology 34 (8), 785803.

Chakraborty, S., Dasgupta, S., Neogi, S., 2007. Nucleation kinetics controlled by chemical overstepping and its tectonic implications: an example from the Sikkim Himalaya. European Journal of Mineralogy 19 (6), 791-803. 
Chakraborty, S., Ganguly, J., 1992. Cation diffusion in aluminosilicate garnets: experimental determination in spessartine-almandine diffusion couples, evaluation of effective binary diffusion coefficients, and applications. Contributions to Mineralogy and Petrology 111 (1), 74-86.

Chakraborty, S., Mukhopadhyay, D. K., Chowdhury, P., Rubatto, D., Anczkiewicz, R., Trepmann, C., Gaidies, F., Sorcar, N., Dasgupta, S., 2017. Channel flow and localized fault bounded slice tectonics (LFBST): Insights from petrological, structural, geochronological and geospeedometric studies in the Sikkim Himalaya, NE India. Lithos 282, 464-482.

Cherniak, D., 2005. Uranium and manganese diffusion in apatite. Chemical Geology 219 (1-4), 297-308.

Cherniak, D., Dimanov, A., 2010. Diffusion in pyroxene, mica and amphibole. Reviews in Mineralogy and Geochemistry 72 (1), 641-690.

Chernoff, C., Carlson, W., 1997. Disequilibrium for Ca during growth of pelitic garnet. Journal of Metamorphic Geology 15 (4), 421-438.

Chinner, G., 1967. Chloritoid, and the isochemical character of Barrow's zones. Journal of Petrology 8 (2), 268-282.

Christian, J. W., 2002. The Theory of Transformations in Metals and Alloys. Elsevier.

Chu, X., Ague, J., 2015a. A new statistical analysis of rare earth element diffusion data in garnet. In: AGU Fall Meeting Abstracts.

Chu, X., Ague, J. J., 2015b. Analysis of experimental data on divalent cation diffusion kinetics in aluminosilicate garnets with application to timescales of peak Barrovian metamorphism, Scotland. Contributions to Mineralogy and Petrology 170 (2), 25.

Coggan, R., Holland, T., 2002. Mixing properties of phengitic micas and revised garnet-phengite thermometers. J Met Geol 20, 683-696.

Cohen, M., 1970. Self-diffusion during plastic deformation. Transactions of the Japan Institute of Metals 11 (3), 145-151.

Connolly, J., Cesare, B., 1993. C-O-H-S fluid composition and oxygen fugacity in graphitic metapelites. Journal of Metamorphic Geology 11 (3), 379-379.

Corrie, S. L., Kohn, M. J., 2008. Trace-element distributions in silicates during prograde metamorphic reactions: Implications for monazite formation. Journal of Metamorphic Geology 26 (4), 451-464. 
Cosgrove, J. W., 1972. The development and interrelationship of microfolds and crenulation cleavage.

Costa, F., Chakraborty, S., 2008. The effect of water on Si and O diffusion rates in olivine and implications for transport properties and processes in the upper mantle. Physics of the Earth and Planetary Interiors 166 (1-2), 11-29.

Cottle, J., Waters, D., Riley, D., Beyssac, O., Jessup, M., 2011. Metamorphic history of the South Tibetan detachment system, Mt. Everest region, revealed by RSCM thermometry and phase equilibria modelling. Journal of Metamorphic Geology 29 (5), 561-582.

Cruz, M. D. R., 2011. Origin of atoll garnet in schists from the Alpujárride Complex (Central zone of the Betic Cordillera, Spain): implications on the PT evolution. Mineralogy and Petrology 101 (3-4), 245-261.

Daniel, C., Spear, F., 1999. The clustered nucleation and growth processes of garnet in regional metamorphic rocks from north-west Connecticut, USA. Journal of Metamorphic Geology 17 (5), 503-520.

Daniel, C. G., Spear, F. S., 1998. Three-dimensional patterns of garnet nucleation and growth. Geology 26 (6), 503-506.

Darken, L. S., Gurry, R. W., 1953. Physical chemistry of metals. CBS Publishers and Distributors.

Dasgupta, S., Chakraborty, S., Neogi, S., 2009. Petrology of an inverted Barrovian sequence of metapelites in Sikkim Himalaya, India: constraints on the tectonics of inversion. American Journal of Science 309 (1), 43-84.

Dasgupta, S., Ganguly, J., Neogi, S., 2004. Inverted metamorphic sequence in the Sikkim Himalayas: crystallization history, $\mathrm{P}-\mathrm{T}$ gradient and implications. Journal of Metamorphic Geology 22 (5), 395-412.

de Capitani, C., Brown, T. H., 1987. The computation of chemical equilibrium in complex systems containing non-ideal solutions. Geochimica et Cosmochimica Acta 51 (10), 2639-2652.

de Capitani, C., Petrakakis, K., 2010. The computation of equilibrium assemblage diagrams with Theriak/Domino software. American Mineralogist 95 (7), 1006-1016. 
de Sigoyer, J., Chavagnac, V., Blichert-Toft, J., Villa, I. M., Luais, B., Guillot, S., Cosca, M., Mascle, G., 2000. Dating the Indian continental subduction and collisional thickening in the northwest Himalaya: Multichronology of the Tso Morari eclogites. Geology 28 (6), 487-490.

Dempster, T. J., Symon, S., Chung, P., 2017. Intergranular diffusion rates from the analysis of garnet surfaces: Implications for metamorphic equilibration. Journal of Metamorphic Geology.

Denison, C., Carlson, W., 1997. Three-dimensional quantitative textural analysis of metamorphic rocks using high-resolution computed X-ray tomography: Part ii. Application to natural samples. Journal of Metamorphic Geology 15 (1), 45-57.

DeVore, G. W., 1955. Crystal growth and the distribution of elements. The Journal of Geology 63 (5), 471-494.

Dewers, T., Ortoleva, P., 1989. Self-organization of mineralization patterns in metamorphic rocks through mechanochemical coupling. The Journal of Physical Chemistry 93 (7), 2842-2848.

Dewers, T., Ortoleva, P., 1990. Geochemical self-organization III: A mechano-chemical model of metamorphic differentiation. Am. J. Sci 290, 473-521.

Dobbs, H. T., Peruzzo, L., Seno, F., Spiess, R., Prior, D. J., 2003. Unraveling the Schneeberg garnet puzzle: a numerical model of multiple nucleation and coalescence. Contributions to Mineralogy and Petrology 146 (1), 1-9.

Dohmen, R., Chakraborty, S., 2003. Mechanism and kinetics of element and isotopic exchange mediated by a fluid phase. American Mineralogist 88 (8-9), 1251-1270.

Dohmen, R., Milke, R., 2010. Diffusion in polycrystalline materials: grain boundaries, mathematical models, and experimental data. Reviews in Mineralogy and Geochemistry 72 (1), 921-970.

Dragovic, B., Baxter, E. F., Caddick, M. J., 2015. Pulsed dehydration and garnet growth during subduction revealed by zoned garnet geochronology and thermodynamic modeling, Sifnos, Greece. Earth and Planetary Science Letters 413, 111-122.

Dragovic, B., Samanta, L. M., Baxter, E. F., Selverstone, J., 2012. Using garnet to constrain the duration and rate of water-releasing metamorphic reactions during subduction: an example from Sifnos, Greece. Chemical Geology 314, 9-22.

Drury, M. R., Urai, J. L., 1990. Deformation-related recrystallization processes. Tectonophysics 172 (3-4), 235-253. 
Dubey, C. S., Catlos, E., Sharma, B., 2005. Modelling of PTt paths constrained by mineral chemistry and monazite dating of metapelites in relationship to MCT activity in Sikkim Eastern Himalayas. Metamorphism and Crustal Evolution: New Delhi, Atlantic Publishers, 250-282.

Duchêne, S., Blichert-Toft, J., Luais, B., Télouk, P., et al., 1997. The Lu-Hf dating of garnets and the ages of the Alpine high-pressure metamorphism. Nature 387 (6633), 586.

Edmunds, W., Atherton, M., 1971. Polymetamorphic evolution of garnet in the Fanad aureole, Donegal, Eire. Lithos 4 (2), 147-161.

Faak, K., Chakraborty, S., Dasgupta, S., 2012. Petrology and tectonic significance of metabasite slivers in the Lesser and Higher Himalayan domains of Sikkim, India. Journal of metamorphic geology 30 (6), 599-622.

Faccenda, M., Gerya, T. V., Chakraborty, S., 2008. Styles of post-subduction collisional orogeny: influence of convergence velocity, crustal rheology and radiogenic heat production. Lithos 103 (1-2), 257-287.

Farver, J. R., 1994. Oxygen self-diffusion in calcite: dependence on temperature and water fugacity. Earth and Planetary Science Letters 121 (3-4), 575-587.

Farver, J. R., Yund, R., 1991. Oxygen diffusion in quartz: dependence on temperature and water fugacity. Chemical geology 90 (1-2), 55-70.

Farver, J. R., Yund, R. A., 1992. Oxygen diffusion in a fine-grained quartz aggregate with wetted and nonwetted microstructures. Journal of Geophysical Research: Solid Earth 97 (B10), 14017-14029.

Faryad, S. W., Chakraborty, S., 2005. Duration of Eo-Alpine metamorphic events obtained from multicomponent diffusion modeling of garnet: a case study from the Eastern Alps. Contributions to Mineralogy and Petrology 150 (3), 306-318.

Fei, H., Wiedenbeck, M., Yamazaki, D., Katsura, T., 2013. Small effect of water on upper-mantle rheology based on silicon self-diffusion coefficients. Nature 498 (7453), 213.

Finlay, C., Kerr, A., 1987. Evidence for differences in growth rate among garnets in pelitic schists from northern Sutherland, Scotland. Mineralogical Magazine 51 (62), 569-576. 
Finlay, C. A., Kerr, A., 1979. Garnet growth in a metapelite from the Moinian rocks of northern Sutherland, Scotland. Contributions to Mineralogy and Petrology 71 (2), $185-191$.

Fisher, G. W., 1973. Nonequilibrium thermodynamics as a model for diffusioncontrolled metamorphic processes. American Journal of Science 273 (10), 897-924.

Fisher, G. W., 1978. Rate laws in metamorphism. Geochimica et Cosmochimica Acta $42(7), 1035-1050$.

Fletcher, R. C., 1977. Quantitative theory for metamorphic differentiation in development of crenulation cleavage. Geology 5 (3), 185-187.

Florence, F. P., Spear, F. S., 1991. Effects of diffusional modification of garnet growth zoning on PT path calculations. Contributions to Mineralogy and Petrology 107 (4), $487-500$.

Foster, C., 1982. Textural variation of sillimanite segregations. The Canadian Mineralogist 20 (3), 379-392.

Gaidies, F., 2017. Nucleation in geological materials. EMU Notes in Mineralogy. European Mineralogical Union, book section 11.

Gaidies, F., Abart, R., De Capitani, C., Schuster, R., Connolly, J., Reusser, E., 2006. Characterization of polymetamorphism in the Austroalpine basement east of the Tauern Window using garnet isopleth thermobarometry. Journal of Metamorphic Geology 24 (6), 451-475.

Gaidies, F., De Capitani, C., Abart, R., 2008. THERIA_G: a software program to numerically model prograde garnet growth. Contributions to Mineralogy and Petrology 155 (5), 657-671.

Gaidies, F., Heinrich, W., Milke, R., Abart, R., 2017. Metamorphic mineral reactions. Vol. 17 of EMU Notes in Mineralogy. European Mineralogical Union, book section 14 .

Gaidies, F., Pattison, D., De Capitani, C., 2011. Toward a quantitative model of metamorphic nucleation and growth. Contributions to Mineralogy and Petrology 162 (5), 975-993.

Gaidies, F., Petley-Ragan, A., Chakraborty, S., Dasgupta, S., Jones, P., 2015. Constraining the conditions of Barrovian metamorphism in Sikkim, India: $\mathrm{P}-\mathrm{T}-\mathrm{t}$ paths of garnet crystallization in the Lesser Himalayan Belt. Journal of Metamorphic Geology $33(1), 23-44$. 
Ganguly, J., 2002. Diffusion kinetics in minerals: principles and applications to tectono-metamophic processes.

Gansser, A. H. . A., 1938. Central Himalaya. Hindustan Publishing; Delhi.

George, F., Gaidies, F., 2017. Characterisation of a garnet population from the Sikkim Himalaya: insights into the rates and mechanisms of porphyroblast crystallisation. Contributions to Mineralogy and Petrology 172 (7), 57.

George, F., Gaidies, F., Boucher, B., 2018. Population-wide garnet growth zoning revealed by LA-ICP-MS mapping: implications for trace element equilibration and syn-kinematic deformation during crystallisation. Contributions to Mineralogy and Petrology 173 (9), 74.

Ghosh, S., Bose, S., Mandal, N., Dasgupta, S., 2016. Dynamic recrystallization mechanisms and their transition in the Daling Thrust (DT) zone, Darjeeling-Sikkim Himalaya. Tectonophysics 674, 166-181.

Goswami, S., 2005. Inverted metamorphism in the Sikkim-Darjeeling Himalaya: structural, metamorphic and numerical studies. Ph.D. thesis, University of Cambridge.

Griera, A., Bons, P. D., Jessell, M. W., Lebensohn, R. A., Evans, L., Gomez-Rivas, E., 2011. Strain localization and porphyroclast rotation. Geology 39 (3), 275-278.

Gupta, S., Das, A., Goswami, S., Modak, A., Mondal, S., 2010. Evidence for structural discordance in the inverted metamorphic sequence of sikkim himalaya: towards resolving the main central thrust controversy. Journal of the Geological Society of India 75 (1), 313-322.

Habler, G., Thöni, M., Grasemann, B., 2009. Cretaceous metamorphism in the Austroalpine Matsch Unit (Eastern Alps): the interrelation between deformation and chemical equilibration processes. Mineralogy and Petrology 97 (3-4), 149.

Hall, R., 1953. Segregation of impurities during the growth of germanium and silicon. The Journal of Physical Chemistry 57 (8), 836-839.

Haskin, L. A., Wildeman, T. R., Frey, F. A., Collins, K. A., Keedy, C. R., Haskin, M. A., 1966. Rare earths in sediments. Journal of Geophysical Research 71 (24), 6091-6105.

Hawkins, A., Selverstone, J., Brearley, A., Beane, R., Ketcham, R., Carlson, W., 2007. Origin and mechanical significance of honeycomb garnet in high-pressure metasedimentary rocks from the Tauern Window, Eastern Alps. Journal of Metamorphic Geology 25 (5), 565-583. 
Heinrich, C., Pettke, T., Halter, W., Aigner-Torres, M., Audétat, A., Günther, D., Hattendorf, B., Bleiner, D., Guillong, M., Horn, I., 2003. Quantitative multielement analysis of minerals, fluid and melt inclusions by laser-ablation inductivelycoupled-plasma mass-spectrometry. Geochimica et Cosmochimica Acta 67 (18), 3473-3497.

Heinrich, W., Rehs, G., Franz, G., 1997. Monazite-xenotime miscibility gap thermometry. I. An empirical calibration. Journal of Metamorphic Geology 15 (1), $3-16$.

Hellstrom, J., Paton, C., Woodhead, J., Hergt, J., 2008. Iolite: software for spatially resolved LA-(quad and MC) ICPMS analysis. Mineralogical Association of Canada short course series 40, 343-348.

Henry, D. J., Guidotti, C. V., Thomson, J. A., 2005. The Ti-saturation surface for low-to-medium pressure metapelitic biotites: Implications for geothermometry and Ti-substitution mechanisms. American Mineralogist 90 (2-3), 316-328.

Herrmann, G., Gleiter, H., Bäro, G., 1976. Investigation of low energy grain boundaries in metals by a sintering technique. Acta Metallurgica 24 (4), 353-359.

Hickey, K., Bell, T., 1999. Behaviour of rigid objects during deformation and metamorphism: a test using schists from the Bolton syncline, Connecticut, USA. Journal of Metamorphic Geology 17, 211-228.

Hickmott, D., Shimizu, N., Spear, F., Selverstone, J., 1987. Trace-element zoning in a metamorphic garnet. Geology 15 (6), 573-576.

Hickmott, D., Spear, F. S., 1992. Major-and trace-element zoning in garnets from calcareous pelites in the NW Shelburne Falls Quadrangle, Massachusetts: garnet growth histories in retrograded rocks. Journal of Petrology 33 (5), 965-1005.

Hickmott, D., Treves, B., Roselle, G., Baumgartner, L., 1997. Micro-PIXE analysis of carbonates and silicates: tracking fluid flow in crustal environments. Nuclear Instruments and Methods in Physics Research Section B: Beam Interactions with Materials and Atoms 130 (1-4), 660-665.

Hickmott, D. D., Shimizu, N., 1990. Trace element zoning in garnet from the Kwoiek Area, British Columbia: disequilibrium partitioning during garnet growth? Contributions to Mineralogy and Petrology 104 (6), 619-630.

Hielscher, R., Schaeben, H., 2008. A novel pole figure inversion method: specification of the MTEX algorithm. Journal of Applied Crystallography 41 (6), 1024-1037. 
Hirsch, D. M., 2008. Controls on porphyroblast size along a regional metamorphic field gradient. Contributions to Mineralogy and Petrology 155 (4), 401-415.

Hirsch, D. M., 2011. Reduce3D: A tool for three-dimensional spatial statistical analysis of crystals. Geosphere 7 (3), 724-732.

Hirsch, D. M., Prior, D. J., Carlson, W. D., 2003. An overgrowth model to explain multiple, dispersed high-Mn regions in the cores of garnet porphyroblasts. American Mineralogist 88 (1), 131-141.

Hobbs, B. E., Ord, A., Regenauer-Lieb, K., 2011. The thermodynamics of deformed metamorphic rocks: a review. Journal of Structural Geology 33 (5), 758-818.

Holland, T., Powell, R., 1998. An internally consistent thermodynamic data set for phases of petrological interest. Journal of metamorphic Geology 16 (3), 309-343.

Holm, D. K., Selverstone, J., 1990. Rapid growth and strain rates inferred from synkinematic garnets, Penokean orogeny, Minnesota. Geology 18 (2), 166-169.

Hoschek, G., 1969. The stability of staurolite and chloritoid and their significance in metamorphism of pelitic rocks. Contributions to Mineralogy and Petrology 22 (3), $208-232$.

Ildefonse, B., Sokoutis, D., Mancktelow, N. S., 1992. Mechanical interactions between rigid particles in a deforming ductile matrix. Analogue experiments in simple shear flow. Journal of Structural Geology 14 (10), 1253-1266.

Jackson, S. E., Longerich, H. P., Dunning, G. R., Freyer, B. J., 1992. The application of laser-ablation microprobe inductively coupled plasma-mass spectrometry (LAM-ICP-MS) to in situ trace-element determinations in minerals. The Canadian Mineralogist 30 (4), 1049-1064.

Janots, E., Engi, M., Berger, A., Allaz, J., Schwarz, J.-O., Spandler, C., 2008. Prograde metamorphic sequence of REE minerals in pelitic rocks of the Central Alps: implications for allanite-monazite-xenotime phase relations from 250 to $610{ }^{\circ} \mathrm{C}$. Journal of Metamorphic Geology 26 (5), 509-526.

Jessell, M., Bons, P., Evans, L., Barr, T., Stüwe, K., 2001. Elle: the numerical simulation of metamorphic and deformation microstructures. Computers \& Geosciences 27 (1), 17-30.

Joesten, R., 1991. Grain-boundary diffusion kinetics in silicate and oxide minerals. In: Diffusion, atomic ordering, and mass transport. Springer, pp. 345-395. 
Johnson, S., Dupee, M., Guidotti, C., 2006. Porphyroblast rotation during crenulation cleavage development: an example from the aureole of the Mooselookmeguntic pluton, Maine, USA. Journal of Metamorphic Geology 24 (1), 55-73.

Johnson, S. E., 2009. Porphyroblast rotation and strain localization: Debate settled! Geology 37 (7), 663-666.

Jung, S., Hellebrand, E., 2006. Trace element fractionation during high-grade metamorphism and crustal melting - constraints from ion microprobe data of metapelitic, migmatitic and igneous garnets and implications for $\mathrm{Sm}-\mathrm{Nd}$ garnet chronology. Lithos 87 (3), 193-213.

Kamb, W. B., 1959. Theory of preferred crystal orientation developed by crystallization under stress. The Journal of Geology 67 (2), 153-170.

Keller, L., Wirth, R., Rhede, D., Kunze, K., Abart, R., 2008. Asymmetrically zoned reaction rims: assessment of grain boundary diffusivities and growth rates related to natural diffusion-controlled mineral reactions. Journal of Metamorphic Geology 26 (1), 99-120.

Kelly, E., Carlson, W., Connelly, J., 2011. Implications of garnet resorption for the $\mathrm{Lu}-\mathrm{Hf}$ garnet geochronometer: an example from the contact aureole of the Makhavinekh Lake Pluton, Labrador. Journal of Metamorphic Geology 29 (8), 901-916.

Kelly, E., Carlson, W., Ketcham, R., 2013. Magnitudes of departures from equilibrium during regional metamorphism of porphyroblastic rocks. Journal of Metamorphic Geology 31 (9), 981-1002.

Kessel, R., Schmidt, M. W., Ulmer, P., Pettke, T., 2005. Trace element signature of subduction-zone fluids, melts and supercritical liquids at 120-180 km depth. Nature 437 (7059), 724.

Ketcham, R. A., 2005. Computational methods for quantitative analysis of threedimensional features in geological specimens. Geosphere 1 (1), 32-41.

Ketcham, R. A., Carlson, W. D., 2001. Acquisition, optimization and interpretation of X-ray computed tomographic imagery: applications to the geosciences. Computers \&amp; Geosciences 27 (4), 381-400.

Kohn, M. J., 2004. Oscillatory- and sector-zoned garnets record cyclic (?) rapid thrusting in central Nepal. Geochemistry, Geophysics, Geosystems 5 (12). 
Kohn, M. J., 2009. Models of garnet differential geochronology. Geochimica et Cosmochimica Acta 73 (1), 170-182.

Kohn, M. J., Corrie, S. L., Markley, C., 2015. The fall and rise of metamorphic zircon. American Mineralogist 100 (4), 897-908.

Kohn, M. J., Malloy, M. A., 2004. Formation of monazite via prograde metamorphic reactions among common silicates: implications for age determinations. Geochimica et Cosmochimica Acta 68 (1), 101-113.

Kohn, M. J., Penniston-Dorland, S. C., 2017. Diffusion: Obstacles and opportunities in petrochronology. Reviews in Mineralogy and Geochemistry 83 (1), 103-152.

Konrad-Schmolke, M., Halama, R., 2014. Combined thermodynamic-geochemical modeling in metamorphic geology: Boron as tracer of fluid-rock interaction. Lithos 208, 393-414.

Konrad-Schmolke, M., Handy, M. R., Babist, J., O’Brien, P. J., 2005. Thermodynamic modelling of diffusion-controlled garnet growth. Contributions to Mineralogy and Petrology 149 (2), 181-195.

Konrad-Schmolke, M., O’Brien, P. J., de Capitani, C., Carswell, D. A., 2008. Garnet growth at high-and ultra-high pressure conditions and the effect of element fractionation on mineral modes and composition. Lithos 103 (3), 309-332.

Kretz, R., 1966. Interpretation of the shape of mineral grains in metamorphic rocks. Journal of Petrology 7 (1), 68-94.

Kretz, R., 1969. On the spatial distribution of crystals in rocks. Lithos 2 (1), 39-65.

Kretz, R., 1973. Kinetics of the crystallization of garnet at two localities near yellowknife. The Canadian Mineralogist 12 (1), 1-20.

Kretz, R., 1974. Some models for the rate of crystallization of garnet in metamorphic rocks. Lithos 7 (3), 123-131.

Kretz, R., 1993. A garnet population in Yellowknife schist, Canada. Journal of Metamorphic Geology 11 (1), 101-120.

Lanari, P., Riel, N., Guillot, S., Vidal, O., Schwartz, S., Pêcher, A., Hattori, K. H., 2013. Deciphering high-pressure metamorphism in collisional context using microprobe mapping methods: Application to the Stak eclogitic massif (northwest Himalaya). Geology 41 (2), 111-114. 
Lanzirotti, A., 1995. Yttrium zoning in metamorphic garnets. Geochimica et Cosmochimica Acta 59 (19), 4105-4110.

Lapen, T. J., Johnson, C. M., Baumgartner, L. P., Mahlen, N. J., Beard, B. L., Amato, J. M., 2003. Burial rates during prograde metamorphism of an ultra-highpressure terrane: an example from Lago di Cignana, western Alps, Italy. Earth and Planetary Science Letters 215 (1), 57-72.

Lasaga, A. C., 1979. Multicomponent exchange and diffusion in silicates. Geochimica et Cosmochimica Acta 43 (4), 455-469.

Lasaga, A. C., 1981. Rate laws of chemical reactions. Rev. Mineral.;(United States) 8.

Lasaga, A. C., 1983. Geospeedometry: an extension of geothermometry. In: Kinetics and equilibrium in mineral reactions. Springer, pp. 81-114.

Lasaga, A. C., 1986. Metamorphic reaction rate laws and development of isograds. Mineralogical Magazine 50 (3, 357), 359-373.

Lasaga, A. C., Kirkpatrick, J., 1981. Kinetics of geochemical processes. Vol. 8. Walter de Gruyter GmbH \& Co KG.

Le Fort, P., 1975. Himalayas: the collided range. Present knowledge of the continental arc. American Journal of Science 275 (1), 44.

Lee, J. K., Barnett, D., Aaronson, H., 1977. The elastic strain energy of coherent ellipsoidal precipitates in anisotropic crystalline solids. Metallurgical Transactions A 8 (6), 963-970.

Lifshitz, I. M., Slyozov, V. V., 1961. The kinetics of precipitation from supersaturated solid solutions. Journal of physics and chemistry of solids 19 (1-2), 35-50.

Loomis, T. P., 1982. Numerical simulations of crystallization processes of plagioclase in complex melts: the origin of major and oscillatory zoning in plagioclase. Contributions to Mineralogy and Petrology 81 (3), 219-229.

Loomis, T. P., Ganguly, J., Elphick, S. C., 1985. Experimental determination of cation diffusivities in aluminosilicate garnets. Contributions to Mineralogy and Petrology 90 (1), 45-51.

Mackenzie, J., 1958. Second paper on statistics associated with the random disorientation of cubes. Biometrika 45 (1-2), 229-240. 
Mahar, E. M., Baker, J., Powell, R., Holland, T., Howell, N., 1997. The effect of mn on mineral stability in metapelites. Journal of Metamorphic Geology 15 (2), $223-238$.

Mainprice, D., Hielscher, R., Schaeben, H., 2011. Calculating anisotropic physical properties from texture data using the MTEX open-source package. Geological Society, London, Special Publications 360 (1), 175-192.

Mallett, F., 1875. On the geology and mineral resources of Darjeeling district and Western Duars. Memoirs of the Geological Survey of India, no. 11.

Manning, C. E., Ingebritsen, S. E., Bird, D. K., 1993. Missing mineral zones in contact metamorphosed basalts. American Journal of Science 293 (9), 894-938.

Martin, A. J., 2009. Sub-millimeter heterogeneity of yttrium and chromium during growth of semi-pelitic garnet. Journal of Petrology 50 (9), 1713-1727.

Martin, A. J., DeCelles, P. G., Gehrels, G. E., Patchett, P. J., Isachsen, C., 2005. Isotopic and structural constraints on the location of the Main Central thrust in the Annapurna Range, central Nepal Himalaya. Geological Society of America Bulletin 117 (7-8), 926-944.

McQuarrie, N., Robinson, D., Long, S., Tobgay, T., Grujic, D., Gehrels, G., Ducea, M., 2008. Preliminary stratigraphic and structural architecture of Bhutan: Implications for the along strike architecture of the Himalayan system. Earth and Planetary Science Letters 272 (1-2), 105-117.

McQuarrie, N., Tobgay, T., Long, S. P., Reiners, P. W., Cosca, M. A., 2014. Variable exhumation rates and variable displacement rates: Documenting recent slowing of Himalayan shortening in western Bhutan. Earth and Planetary Science Letters 386, $161-174$.

Menard, T., Spear, F. S., 1996. Interpretation of plagioclase zonation in calcic pelitic schist, south Strafford, Vermont, and the effects on thermobarometry. The Canadian Mineralogist 34 (1), 133-146.

Mitra, G., Bhattacharyya, K., Mukul, M., 2010. The Lesser Himalayan duplex in Sikkim: Implications for variations in Himalayan shortening. Journal of the Geological Society of India 75 (1), 289-301.

Miyazaki, K., 1991. Ostwald ripening of garnet in high $\mathrm{P} / \mathrm{T}$ metamorphic rocks. Contributions to Mineralogy and Petrology 108 (1-2), 118-128. 
Miyazaki, K., 1996. A numerical simulation of textural evolution due to Ostwald ripening in metamorphic rocks: A case for small amount of volume of dispersed crystals. Geochimica et cosmochimica acta 60 (2), 277-290.

Miyazaki, K., 2000. The case against Ostwald ripening of porphyroblasts: discussion. Canadian Mineralogist 38 (4), 1027-1028.

Miyazaki, K., 2015. Diffusion-controlled growth and degree of disequilibrium of garnet porphyroblasts: is diffusion-controlled growth of porphyroblasts common? Progress in Earth and Planetary Science 2 (1), 25.

Mohan, A., Windley, B., Searle, M., 1989. Geothermobarometry and development of inverted metamorphism in the Darjeeling-Sikkim region of the eastern Himalayan. Journal of Metamorphic Geology 7 (1), 95-110.

Moore, S., Carlson, W., Hesse, M., 2013. Origins of yttrium and rare earth element distributions in metamorphic garnet. Journal of Metamorphic Geology 31 (6), 663689.

Moore, S. J., Carlson, W. D., 2015. Evaluation of a combined HRXCT/EBSD method for detecting epitaxial nucleation of garnet porphyroblasts. European Journal of Mineralogy 27 (1), 19-29.

Mottram, C. M., Argles, T., Harris, N., Parrish, R., Horstwood, M., Warren, C., Gupta, S., 2014a. Tectonic interleaving along the Main Central Thrust, Sikkim Himalaya. Journal of the Geological Society 171 (2), 255-268.

Mottram, C. M., Warren, C. J., Regis, D., Roberts, N. M., Harris, N. B., Argles, T. W., Parrish, R. R., 2014b. Developing an inverted Barrovian sequence; insights from monazite petrochronology. Earth and Planetary Science Letters 403, 418-431.

Mukhopadhyay, D. K., Chakraborty, S., Trepmann, C., Rubatto, D., Anczkiewicz, R., Gaidies, F., Dasgupta, S., Chowdhury, P., 2017. The nature and evolution of the Main Central Thrust: Structural and geochronological constraints from the Sikkim Himalaya, NE India. Lithos 282, 447-463.

Mukul, M., 2000. The geometry and kinematics of the Main Boundary Thrust and related neotectonics in the Darjiling Himalayan fold-and-thrust belt, West Bengal, India. Journal of Structural Geology 22 (9), 1261-1283.

Mukul, M., Jaiswal, M., Singhvi, A., 2007. Timing of recent out-of-sequence active deformation in the frontal Himalayan wedge: Insights from the Darjiling subHimalaya, India. Geology 35 (11), 999-1002. 
Müller, W., Aerden, D., Halliday, A. N., 2000. Isotopic dating of strain fringe increments: Duration and rates of deformation in shear zones. Science 288 (5474), 2195-2198.

Neogi, S., Dasgupta, S., Fukuoka, M., 1998. High P-T polymetamorphism, dehydration melting, and generation of migmatites and granites in the Higher Himalayan Crystalline Complex, Sikkim, India. Journal of Petrology 39 (1), 61-99.

Norman, M., Pearson, N., Sharma, A., Griffin, W., 1996. Quantitative analysis of trace elements in geological materials by laser ablation ICPMS: instrumental operating conditions and calibration values of NIST glasses. Geostandards and Geoanalytical Research 20 (2), 247-261.

Oliver, G., Chen, F., Buchwaldt, R., Hegner, E., 2000. Fast tectonometamorphism and exhumation in the type area of the Barrovian and Buchan zones. Geology $28(5), 459-462$.

Orville, P. M., 1962. Alkali metasomatism and feldspars. Norsk Geologisk Tidsskrift 42 (283), e316.

Otamendi, J. E., Jesús, D., Douce, A. E. P., Castro, A., 2002. Rayleigh fractionation of heavy rare earths and yttrium during metamorphic garnet growth. Geology 30 (2), $159-162$.

Palin, R. M., Weller, O. M., Waters, D. J., Dyck, B., 2016. Quantifying geological uncertainty in metamorphic phase equilibria modelling; a Monte Carlo assessment and implications for tectonic interpretations. Geoscience Frontiers 7 (4), 591-607.

Passchier, C., Trouw, R., Zwart, H., Vissers, R., 1992. Porphyroblast rotation: eppur si muove? Journal of Metamorphic Geology 10 (3), 283-294.

Paterson, S., Vernon, R., 2001. Inclusion trail patterns in porphyroblasts from the Foothills Terrane, California: a record of orogenesis or local strain heterogeneity? Journal of Metamorphic Geology 19 (4), 351-372.

Paton, C., Hellstrom, J., Paul, B., Woodhead, J., Hergt, J., 2011. Iolite: Freeware for the visualisation and processing of mass spectrometric data. Journal of Analytical Atomic Spectrometry 26 (12), 2508-2518.

Pattison, D., DeBuhr, C., 2015. Petrology of metapelites in the Bugaboo aureole, British Columbia, Canada. Journal of Metamorphic Geology 33 (5), 437-462. 
Pattison, D. R., De Capitani, C., Gaidies, F., 2011. Petrological consequences of variations in metamorphic reaction affinity. Journal of Metamorphic Geology 29 (9), 953-977.

Pattison, D. R., Tinkham, D., 2009. Interplay between equilibrium and kinetics in prograde metamorphism of pelites: an example from the Nelson aureole, British Columbia. Journal of Metamorphic Geology 27 (4), 249-279.

Paul, B., Paton, C., Norris, A., Woodhead, J., Hellstrom, J., Hergt, J., Greig, A., 2012. Cellspace: a module for creating spatially registered laser ablation images within the Iolite freeware environment. Journal of Analytical Atomic Spectrometry 27 (4), 700-706.

Paul, D., Chandy, K., Bhalla, J., Prasad, N., Sengupta, N., 1982. Geochronology and geochemistry of Lingtse Gneiss, Darjeeling-Sikkim Himalayas. Indian Journal of Earth Sciences 9 (1), 11-17.

Pearce, N. J., Perkins, W. T., Westgate, J. A., Gorton, M. P., Jackson, S. E., Neal, C. R., Chenery, S. P., 1997. A compilation of new and published major and trace element data for NIST SRM 610 and NIST SRM 612 glass reference materials. Geostandards Newsletter 21 (1), 115-144.

Petley-Ragan, A., Gaidies, F., Pattison, D., 2016. A statistical analysis of the distribution of cordierite and biotite in hornfels from the Bugaboo contact aureole: implications for the kinetics of porphyroblast crystallization. Journal of Metamorphic Geology 34 (1), 85-101.

Pollington, A. D., Baxter, E. F., 2011. High precision microsampling and preparation of zoned garnet porphyroblasts for Sm-Nd geochronology. Chemical Geology $281(3), 270-282$.

Powell, D., 1966. On the preferred crystallographic orientation of garnet in some metamorphic rocks. Mineralogical Magazine and Journal of the Mineralogical Society 36 (276), 1094-1109.

Powell, R., Holland, T., Worley, B., 1998. Calculating phase diagrams involving solid solutions via non-linear equations, with examples using THERMOCALC. Journal of Metamorphic Geology 16 (4), 577-588.

Prior, D. J., Wheeler, J., Brenker, F. E., Harte, B., Matthews, M., 2000. Crystal plasticity of natural garnet: New microstructural evidence. Geology 28 (11), 10031006. 
Prior, D. J., Wheeler, J., Peruzzo, L., Spiess, R., Storey, C., 2002. Some garnet microstructures: an illustration of the potential of orientation maps and misorientation analysis in microstructural studies. Journal of Structural Geology 24 (6-7), 999-1011.

Putnis, A., 2002. Mineral replacement reactions: from macroscopic observations to microscopic mechanisms. Mineralogical Magazine 66 (5), 689-708.

Putnis, A., 2014. Why mineral interfaces matter. Science 343 (6178), 1441-1442.

Pyle, J. M., Spear, F. S., 1999. Yttrium zoning in garnet: coupling of major and accessory phases during metamorphic reactions. Geological Materials Research 1 (6), $1-49$.

Pyle, J. M., Spear, F. S., Rudnick, R. L., McDonough, W. F., 2001. Monazitexenotime-garnet equilibrium in metapelites and a new monazite-garnet thermometer. Journal of Petrology 42 (11), 2083-2107.

Raimondo, T., Payne, J., Wade, B., Lanari, P., Clark, C., Hand, M., 2017. Trace element mapping by LA-ICP-MS: assessing geochemical mobility in garnet. Contributions to Mineralogy and Petrology 172 (4), 17.

Ridley, J., Thompson, A., 1986. Fluid-rock interactions during metamorphism:. New York, Springer, Ch. The role of mineral kinetics in the development of metamorphic microtextures, pp. 154-193.

Robin, P.-Y. F., 1979. Theory of metamorphic segregation and related processes. Geochimica et Cosmochimica Acta 43 (10), 1587-1600.

Robinson, D., DeCelles, P., Garzione, C., Pearson, O., Harrison, T., Catlos, E., 2003. Kinematic model for the Main Central Thrust in Nepal. Geology 31 (4), 359-362.

Robyr, M., Carlson, W., Passchier, C., Vonlanthen, P., 2009. Microstructural, chemical and textural records during growth of snowball garnet. Journal of Metamorphic Geology 27 (6), 423-437.

Rocholl, A., 1998. Major and trace element composition and homogeneity of microbeam reference material: Basalt glass USGS BCR-2G. Geostandards newsletter $22(1), 33-45$.

Rosenfeld, J. L., 1970. Rotated garnets in metamorphic rocks. Geological Society of America Special Papers 129, 1-102. 
Sasaki, J., Peterson, N., Hoshino, K., 1985. Tracer impurity diffusion in single-crystal rutile $\left(\mathrm{TiO}_{2}-x\right)$. Journal of Physics and Chemistry of Solids 46 (11), 1267-1283.

Scherer, E. E., Cameron, K. L., Blichert-Toft, J., 2000. Lu-Hf garnet geochronology: closure temperature relative to the $\mathrm{Sm}-\mathrm{Nd}$ system and the effects of trace mineral inclusions. Geochimica et Cosmochimica Acta 64 (19), 3413-3432.

Schoneveld, C., 1977. A study of some typical inclusion patterns in strongly paracrystalline-rotated garnets. Tectonophysics 39 (1-3), 453-471.

Searle, M. P., Szulc, A. G., 2005. Channel flow and ductile extrusion of the high Himalayan slab-the Kangchenjunga-Darjeeling profile, Sikkim Himalaya. Journal of Asian Earth Sciences 25 (1), 173-185.

Shore, M., Fowler, A. D., 1996. Oscillatory zoning in minerals: a common phenomenon. Canadian Mineralogist 34, 1111-1126.

Sinha-Roy, S., 1982. Himalayan Main Central Thrust and its implications for Himalayan inverted metamorphism. Tectonophysics 84 (2-4), 197-224.

Skora, S., Baumgartner, L. P., Mahlen, N. J., Johnson, C. M., Pilet, S., Hellebrand, E., 2006. Diffusion-limited REE uptake by eclogite garnets and its consequences for Lu-Hf and Sm-Nd geochronology. Contributions to Mineralogy and Petrology 152 (6), 703-720.

Skora, S., Lapen, T. J., Baumgartner, L. P., Johnson, C. M., Hellebrand, E., Mahlen, N. J., 2009. The duration of prograde garnet crystallization in the UHP eclogites at Lago di Cignana, Italy. Earth and Planetary Science Letters 287 (3), 402-411.

Smit, M. A., Scherer, E. E., Mezger, K., 2013. Lu-Hf and Sm-Nd garnet geochronology: Chronometric closure and implications for dating petrological processes. Earth and Planetary Science Letters 381, 222-233.

Smye, A. J., Bickle, M. J., Holland, T. J., Parrish, R. R., Condon, D. J., 2011. Rapid formation and exhumation of the youngest Alpine eclogites: a thermal conundrum to Barrovian metamorphism. Earth and Planetary Science Letters 306 (3), 193-204.

Soula, J.-C., Debat, P., 1976. Développement et caractères des litages tectoniques. Bulletin de la Société Géologique de France 7 (6), 1515-1537.

Spear, F. S., Daniel, C. G., 1998. Three-dimensional imaging of garnet porphyroblast sizes and chemical zoning: Nucleation and growth history in the garnet zone. Geological Materials Research 1 (1), 1-44. 
Spear, F. S., Daniel, C. G., 2001. Diffusion control of garnet growth, Harpswell Neck, Maine, USA. Journal of Metamorphic Geology 19 (2), 179-195.

Spear, F. S., Selverstone, J., 1983. Quantitative PT paths from zoned minerals: theory and tectonic applications. Contributions to Mineralogy and Petrology 83 (3-4), 348357.

Spear, F. S., Selverstone, J., Hickmott, D., Crowley, P., Hodges, K. V., 1984. PT paths from garnet zoning: a new technique for deciphering tectonic processes in crystalline terranes. Geology 12 (2), 87-90.

Spear, F. S., Thomas, J. B., Hallett, B. W., 2014. Overstepping the garnet isograd: a comparison of QuiG barometry and thermodynamic modeling. Contributions to Mineralogy and Petrology 168 (3), 1-15.

Spiess, R., Groppo, C., Compagnoni, R., 2007. When epitaxy controls garnet growth. Journal of Metamorphic Geology 25 (4), 439-450.

Spiess, R., Peruzzo, L., Prior, D., Wheeler, J., 2001. Development of garnet porphyroblasts by multiple nucleation, coalescence and boundary misorientation-driven rotations. Journal of Metamorphic Geology 19 (3), 269-290.

Spry, A., 1969. Metamorphic textures, 350 pp.

Stöckhert, B., Massonne, H.-J., Nowlan, E. U., 1997. Low differential stress during high-pressure metamorphism: the microstructural record of a metapelite from the Eclogite Zone, Tauern Window, Eastern Alps. Lithos 41 (1-3), 103-118.

Storey, C., Prior, D., 2005. Plastic deformation and recrystallization of garnet: a mechanism to facilitate diffusion creep. Journal of Petrology 46 (12), 2593-2613.

Stowell, H., Zuluaga, C., Boyle, A., Bulman, G., 2011. Garnet sector and oscillatory zoning linked with changes in crystal morphology during rapid growth, North Cascades, Washington. American Mineralogist 96 (8-9), 1354-1362.

Stowell, H. H., Menard, T., Ridgway, C. K., 1996. Ca-metasomatism and chemical zonation of garnet in contact-metamorphic aureoles, Juneau Gold Belt, southeastern Alaska. The Canadian Mineralogist 34 (6), 1195-1209.

Stünitz, H., 1998. Syndeformational recrystallization - dynamic or compositionally induced? Contributions to Mineralogy and Petrology 131, 219-236. 
Stüwe, K., 1997. Effective bulk composition changes due to cooling: a model predicting complexities in retrograde reaction textures. Contributions to Mineralogy and Petrology 129 (1), 43-52.

Symmes, G. H., Ferry, J. M., 1991. Evidence from mineral assemblages for infiltration of pelitic schists by aqueous fluids during metamorphism. Contributions to Mineralogy and Petrology 108 (4), 419-438.

Talbot, J., Hobbs, B., 1968. The relationship of metamorphic differentiation to other structural features at three localities. The Journal of Geology 76 (5), 581-587.

Teng, H. H., 2013. How ions and molecules organize to form crystals. Elements 9 (3), 189-194.

Thompson, A., 1986. The role of mineral kinetics in the development of metamorphic microtextures. In: Fluid-Rock Interactions during Metamorphism. Springer, pp. $154-193$.

Tiller, W. A., 1991. The science of crystallization: macroscopic phenomena and defect generation. Cambridge University Press.

Trepmann, C. A., Stöckhert, B., 2003. Quartz microstructures developed during nonsteady state plastic flow at rapidly decaying stress and strain rate. Journal of Structural Geology 25 (12), 2035-2051.

Turnbull, D., Fisher, J. C., 1949. Rate of nucleation in condensed systems. The Journal of chemical physics 17 (1), 71-73.

Ulrich, T., Kamber, B. S., Jugo, P. J., Tinkham, D. K., 2009. Imaging elementdistribution patterns in minerals by laser ablation-inductively coupled plasmamass spectrometry (LA-ICP-MS). The Canadian Mineralogist 47 (5), 1001-1012.

Vance, D., Mahar, E., 1998. Pressure-temperature paths from PT pseudosections and zoned garnets: potential, limitations and examples from the Zanskar Himalaya, NW India. Contributions to Mineralogy and Petrology 132 (3), 225-245.

Vidale, R., 1969. Metasomatism in a chemical gradient and the formation of calcsilicate bands. American Journal of Science 267 (8), 857-874.

Vidale, R., 1974. Metamorphic differentiation layering in pelitic rocks of Dutchess County, New York. SAM by BJ GJDLETO H, a YODER, RA 1, 273. 
Vielzeuf, D., Baronnet, A., Perchuk, A., Laporte, D., Baker, M., 2007. Calcium diffusivity in alumino-silicate garnets: an experimental and ATEM study. Contributions to Mineralogy and Petrology 154 (2), 153-170.

Viete, D. R., Hermann, J., Lister, G. S., Stenhouse, I. R., 2011. The nature and origin of the Barrovian metamorphism, Scotland: diffusion length scales in garnet and inferred thermal time scales. Journal of the Geological Society 168 (1), 115-132.

Viete, D. R., Lister, G. S., 2016. On the significance of short-duration regional metamorphism. Journal of the Geological Society, 2016-060.

Voegelé, V., Cordier, P., Sautter, V., Sharp, T., Lardeaux, J., Marques, F., 1998. Plastic deformation of silicate garnets: II. Deformation microstructures in natural samples. Physics of the Earth and Planetary Interiors 108 (4), 319-338.

Volmer, M., Weber, A., 1926. Keimbildung in übersättigten Gebilden. Z. phys. Chem $119,277-301$.

Walther, J. V., Wood, B. J., 1984. Rate and mechanism in prograde metamorphism. Contributions to Mineralogy and Petrology 88 (3), 246-259.

Wang, Z., Ji, S., 1999. Deformation of silicate garnets: Brittle-ductile transition and its geological implications. Canadian Mineralogist 37, 525-541.

Wassmann, S., Stöckhert, B., 2013. Low stress deformation of garnet by incongruent dissolution precipitation creep. Journal of Structural Geology 46, 200-219.

Waters, D., Lovegrove, D., 2002. Assessing the extent of disequilibrium and overstepping of prograde metamorphic reactions in metapelites from the Bushveld Complex aureole, South Africa. Journal of Metamorphic Geology 20 (1), 135-149.

Watson, E. B., 1996. Surface enrichment and trace-element uptake during crystal growth. Geochimica et Cosmochimica Acta 60 (24), 5013-5020.

Watson, E. B., Baxter, E. F., 2007. Diffusion in solid-earth systems. Earth and Planetary Science Letters 253 (3-4), 307-327.

Watson, E. B., Liang, Y., 1995. A simple model for sector zoning in slowly grown crystals: Implications for growth rate and lattice diffusion, with emphasis on accessory minerals in crustal rocks. American Mineralogist 80 (11-12), 1179-1187.

Watson, E. B., Müller, T., 2009. Non-equilibrium isotopic and elemental fractionation during diffusion-controlled crystal growth under static and dynamic conditions. Chemical Geology 267 (3), 111-124. 
Wheeler, J., Prior, D., Jiang, Z., Spiess, R., Trimby, P., 2001. The petrological significance of misorientations between grains. Contributions to mineralogy and petrology 141 (1), 109-124.

White, R., Pomroy, N., Powell, R., 2005. An in situ metatexite--diatexite transition in upper amphibolite facies rocks from Broken Hill, Australia. Journal of Metamorphic Geology 23 (7), 579-602.

White, R., Powell, R., Holland, T., Worley, B., 2000. The effect of $\mathrm{TiO}_{2}$ and $\mathrm{Fe}_{2} \mathrm{O}_{3}$ on metapelitic assemblages at greenschist and amphibolite facies conditions: mineral equilibria calculations in the system $\mathrm{K}_{2} \mathrm{O}-\mathrm{FeO}-\mathrm{MgO}-\mathrm{Al}_{2} \mathrm{O}_{3}-\mathrm{SiO}_{2}-\mathrm{H}_{2} \mathrm{O}-\mathrm{TiO}_{2}-$ $\mathrm{Fe}_{2} \mathrm{O}_{3}$. Journal of Metamorphic Geology 18 (5), 497-512.

Whitney, D. L., Evans, B. W., 2010. Abbreviations for names of rock-forming minerals. American mineralogist 95 (1), 185.

Whitney, D. L., Goergen, E. T., Ketcham, R. A., Kunze, K., 2008. Formation of garnet polycrystals during metamorphic crystallization. Journal of Metamorphic Geology 26 (3), 365-383.

Whitney, D. L., Seaton, N. C., 2010. Garnet polycrystals and the significance of clustered crystallization. Contributions to Mineralogy and Petrology 160 (4), 591607.

Wilbur, D. E., Ague, J. J., 2006. Chemical disequilibrium during garnet growth: Monte Carlo simulations of natural crystal morphologies. Geology 34 (8), 689-692.

Williams, P., 1972. Development of metamorphic layering and cleavage in low grade metamorphic rocks at Bermagui, Australia. American Journal of Science 272 (1), $1-47$.

Wolfe, O. M., Spear, F. S., 2018. Determining the amount of overstepping required to nucleate garnet during Barrovian regional metamorphism, Connecticut Valley Synclinorium. Journal of Metamorphic Geology 36 (1), 79-94.

Woodhead, J. D., Hellstrom, J., Hergt, J. M., Greig, A., Maas, R., 2007. Isotopic and elemental imaging of geological materials by laser ablation inductively coupled plasma-mass spectrometry. Geostandards and Geoanalytical Research 31 (4), 331343.

Yakymchuk, C., Godin, L., 2012. Coupled role of deformation and metamorphism in the construction of inverted metamorphic sequences: an example from farnorthwest Nepal. Journal of Metamorphic Geology 30 (5), 513-535. 
Yang, P., Pattison, D., 2006. Genesis of monazite and Y zoning in garnet from the Black Hills, South Dakota. Lithos 88 (1), 233-253.

Yang, P., Rivers, T., 2001. Chromium and manganese zoning in pelitic garnet and kyanite: spiral, overprint, and oscillatory (?) zoning patterns and the role of growth rate. Journal of Metamorphic Geology 19 (4), 455-474.

Yang, P., Rivers, T., Jackson, S., 1999. Crystal-chemical and thermal controls on trace-element partitioning between coexisting garnet and biotite in metamorphic rocks from western Labrador. The Canadian Mineralogist 37 (2), 443-468.

Zeh, A., 2006. Calculation of garnet fractionation in metamorphic rocks, with application to a flat-top, Y-rich garnet population from the Ruhla Crystalline Complex, Central Germany. Journal of Petrology 47 (12), 2335-2356.

Zener, C., 1949. Theory of growth of spherical precipitates from solid solution. Journal of Applied Physics 20 (10), 950-953.

Zhang, Y., 2010. Diffusion in minerals and melts: theoretical background. Reviews in Mineralogy and Geochemistry 72 (1), 5-59.

Zhao, G., Wilde, S. A., Cawood, P. A., Lu, L., 2000. Petrology and P-T path of the Fuping mafic granulites: implications for tectonic evolution of the central zone of the North China Craton. Journal of Metamorphic Geology 18 (4), 375-391.

Zulauf, G., Helferich, S., 1997. Strain and strain rate in a synkinematic trondhjemitic dike: evidence for melt-induced strain softening during shearing (Bohemian Massif, Czech Republic). Journal of Structural Geology 19 (5), 639-652. 


\section{Appendices 1: Copyright and permissions}

Chapters 2 and 3 were published in the journal Contributions to Mineralogy and Petrology (CPM). Springer Nature holds the copyright for most figures within these chapters. Permission for content reproduction in this thesis was granted and the licenses are attached below. Signed statements of co-authorship of Chapters 2 and 3 are also included. 
This Agreement between Freya George ("You") and Springer Nature ("Springer Nature") consists of your license details and the terms and conditions provided by Springer Nature and Copyright Clearance Center.

Your confirmation email will contain your order number for future reference.

printable details

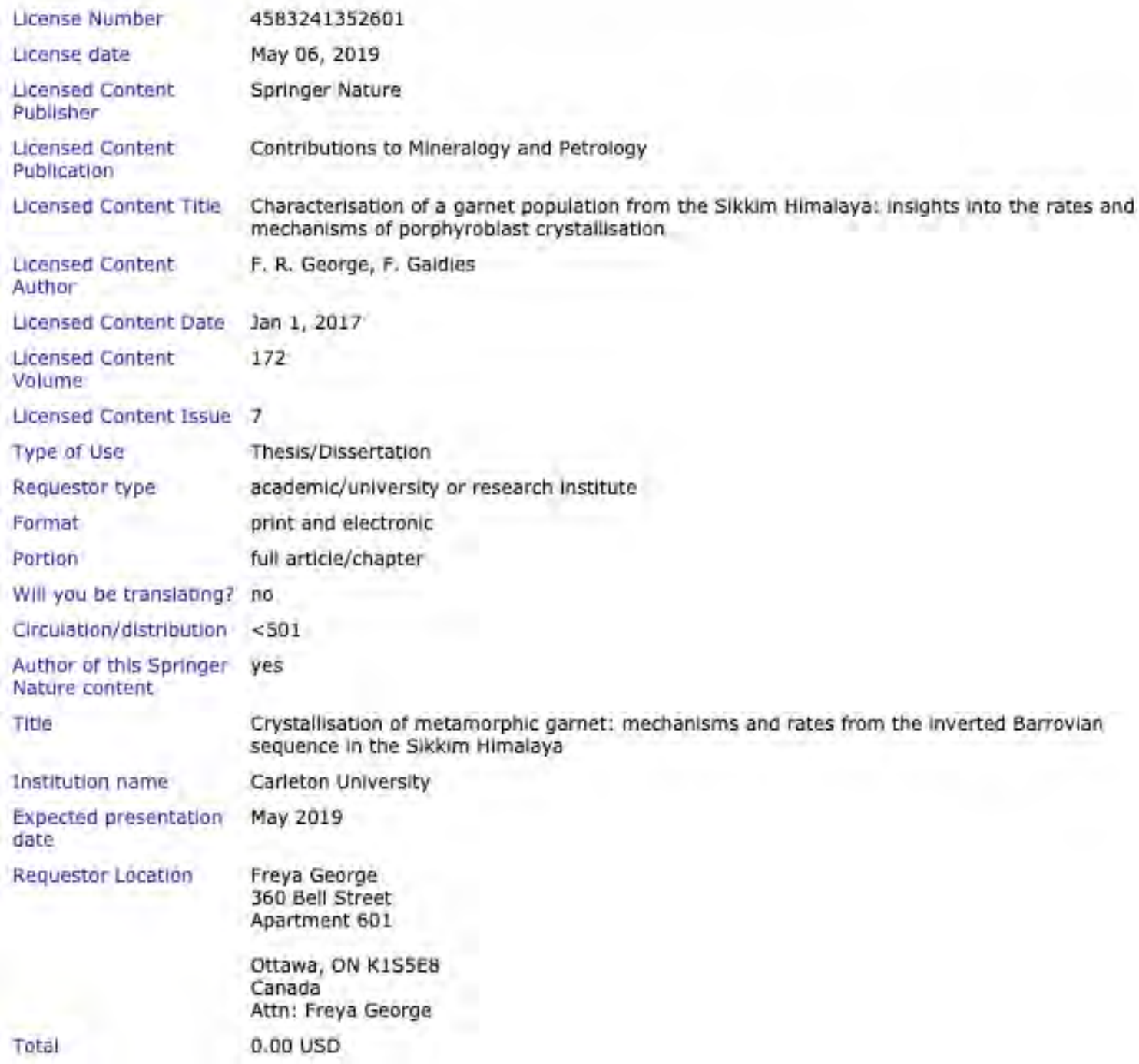


This Agreement between Freya George ("You") and Springer Nature ("Springer Nature") consists of your license details and the terms and conditions provided by Springer Nature and Copyright Clearance Center.

Your confirmation email will contain your order number for future reference.

printable details

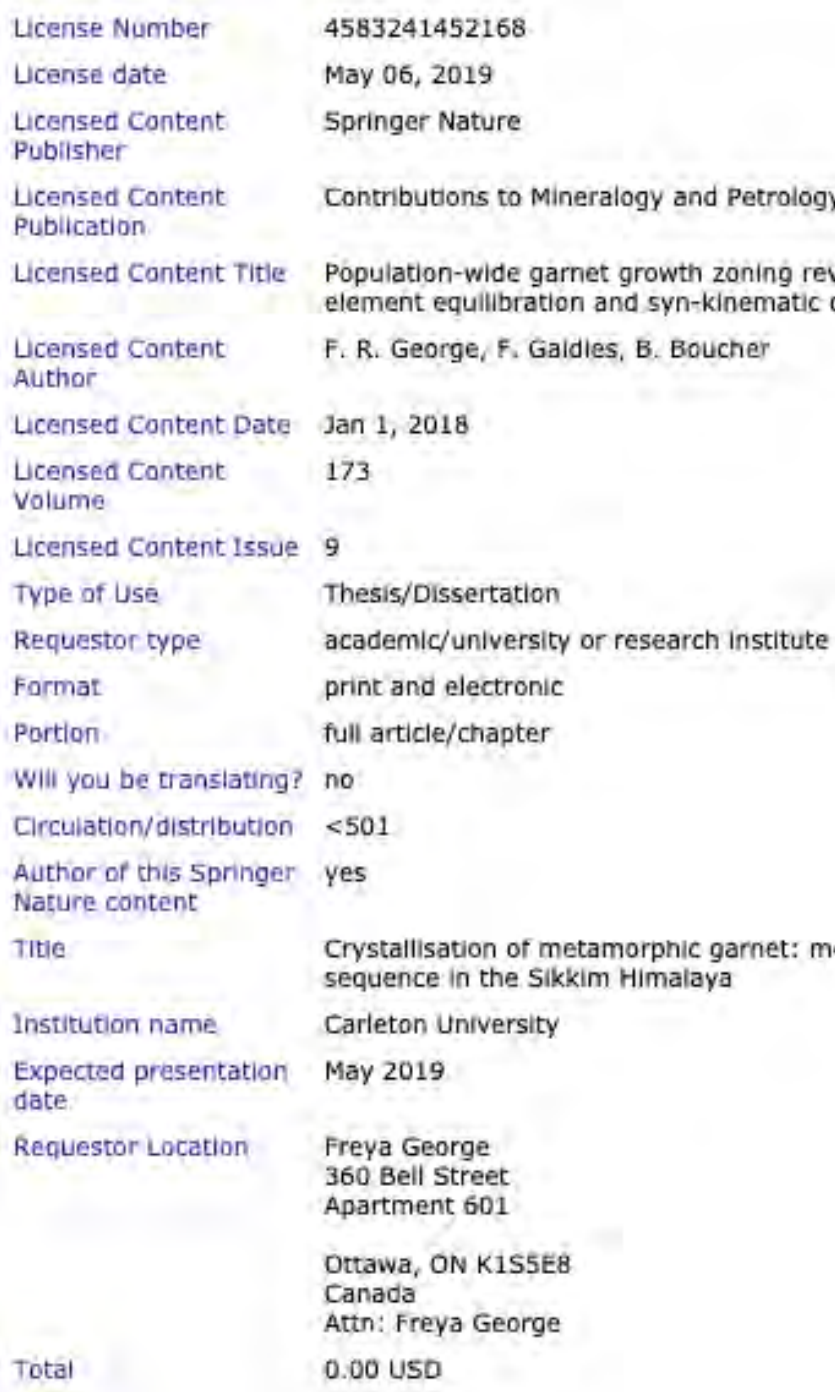




\section{STATEMENT OF CO-AUTHORSHIP}

I, Fred Gaidies, as co-author of "Characterisation of a garnet population from the Sikkim Himalaya: insights into the rates and mechanisms of porphyroblast crystallization", published in the peer-reviewed journal Contributions to Mineralogy and Petrology, and "Population-wide garnet growth zoning revealed by LA-ICP-MS mapping: implications for trace element equilibration and syn-kinematic deformation during crystallization", also published in Contributions to Mineralogy and Petrology, acknowledge Freya Roxanne Georg as the lead author of these manuscripts. Freya designed and performed the studies, obtained and analyzed the data, and wrote and revised the manuscripts.

I, Fred Gaidies, have contributed to the aforementioned published papers in this thesis as supervisor of Freya Roxanne George during her doctoral program in the Department of Earth Sciences at Carleton University. This has entailed support during project development and suggestions, discussion, advice and editorial contributions to the design, fieldwork, analysis and writing of these papers. These activities are consistent with the role of thesis supervisor.

Fred Gaidies 


\section{STATEMENT OF CO-AUTHORSHIP}

I, Brandon Boucher, as co-author of "Population-wide garnet growth zoning revealed by LA-ICP-MS mapping: implications for trace element equilibration and syn-kinematic deformation during crystallization", published in the peerreviewed journal Contributions to Mineralogy and Petrology, acknowledge Freya Roxanne George as the lead contributing author of this manuscript. Freya designed and performed the study, obtained and analyzed all data, and wrote and revised the manuscript.

I, Brandon Boucher, have contributed to the aforementioned paper by overseeing and supporting the collection of laser ablation-inductively coupled plasma-mass spectrometer trace element maps at the University of New Brunswick. This involvement included both primary data processing and support with subsequent re-processing, and editorial revisions of the manuscript. These activities have been entirely consistent with the role of research collaborator in this project.

Brandon Boucher Brash Bouth Date: May 072019 


\section{Appendices 2: Chapter Two}

Appendix 2.1 - Crystal size distribution of 24-99

\begin{tabular}{lccc}
\hline Size class & Radius bin $(\mathrm{mm})$ & Abundance $($ no. garnets) & Abundance $\left(/ \mathrm{cm}^{3}\right)$ \\
\hline 20 & $0.052-0.085$ & 189 & 5.14 \\
19 & $0.086-0.118$ & 820 & 22.29 \\
18 & $0.118-0.152$ & 944 & 27.67 \\
17 & $0.152-0.185$ & 1004 & 27.30 \\
16 & $0.185-0.218$ & 952 & 25.88 \\
15 & $0.218-0.251$ & 884 & 24.03 \\
14 & $0.251-0.284$ & 778 & 21.15 \\
13 & $0.284-0.3187$ & 712 & 19.36 \\
12 & $0.317-0.350$ & 515 & 14.00 \\
11 & $0.350-0.383$ & 425 & 11.56 \\
10 & $0.383-0.416$ & 309 & 8.40 \\
9 & $0.416-0.449$ & 191 & 5.19 \\
8 & $0.449-0.483$ & 58 & 1.58 \\
7 & $0.483-0.516$ & 108 & 2.94 \\
6 & $0.516-0.549$ & 76 & 2.07 \\
5 & $0.549-0.582$ & 61 & 1.66 \\
4 & $0.582-0.615$ & 23 & 0.63 \\
3 & $0.615-0.648$ & 16 & 0.44 \\
2 & $0.648-0.681$ & 4 & 0.11 \\
1 & $0.681-0.714$ & 4 & 0.11 \\
\hline
\end{tabular}




\section{Appendix 2.2 - Chemical composition of mineral phases}

\begin{tabular}{lrrrrrrrr}
\hline Mineral & $\mathrm{Bt}$ & $\mathrm{Pl}$ & $\mathrm{Pl}$ & $\mathrm{Ms}$ & $\mathrm{Chl}$ & $\mathrm{Chl}$ & Ilm & Ilm \\
Occurence & matrix & core & rim & matrix & matrix & grt & matrix & grt \\
no. analyses & 12 & 5 & 4 & 6 & 4 & 4 & 2 & \\
\hline $\mathrm{SiO}_{2}$ & 36.19 & 63.83 & 64.39 & 47.70 & 24.90 & 24.8 & 0.03 & 0.11 \\
$\mathrm{TiO}_{2}$ & 1.52 & 0.00 & 0.00 & 0.25 & 0.09 & 0.10 & 52.29 & 51.54 \\
$\mathrm{Al}_{2} \mathrm{O}_{3}$ & 18.14 & 22.84 & 22.63 & 36.20 & 22.68 & 21.90 & 0.11 & 0.11 \\
$\mathrm{Cr}_{2} \mathrm{O}_{3}$ & 0.02 & 0.00 & 0.00 & 0.02 & 0.01 & 0.01 & 0.01 & 0.00 \\
$\mathrm{FeO}$ & 21.11 & 0.04 & 0.03 & 0.89 & 26.87 & 28.37 & 46.80 & 46.67 \\
$\mathrm{MnO}$ & 0.03 & 0.00 & 0.00 & 0.01 & 0.04 & 0.05 & 0.40 & 0.52 \\
$\mathrm{MgO}$ & 8.43 & 0.00 & 0.00 & 0.58 & 12.97 & 12.27 & 0.02 & 0.30 \\
$\mathrm{CaO}$ & 0.01 & 3.89 & 3.62 & 0.00 & 0.03 & 0.03 & 0.02 & 0.13 \\
$\mathrm{Na} 2 \mathrm{O}$ & 0.23 & 9.48 & 9.59 & 1.79 & 0.07 & 0.05 & 0.00 & 0.00 \\
$\mathrm{~K}_{2} \mathrm{O}$ & 8.67 & 0.09 & 0.09 & 8.63 & 0.04 & 0.10 & 0.00 & 0.00 \\
$\mathrm{Total}$ & 94.35 & 100.16 & 100.34 & 96.08 & 87.70 & 87.67 & 99.67 & 99.48 \\
\hline $\mathrm{Oxygens}$ & 11 & 8 & 8 & 11 & 14 & & 3 & 3 \\
$\mathrm{Si}$ & 2.79 & 2.81 & 2.83 & 3.11 & 2.64 & 2.66 & 0.00 & 0.00 \\
$\mathrm{Ti}$ & 0.09 & 0.00 & 0.00 & 0.01 & 0.01 & 0.01 & 1.00 & 0.99 \\
$\mathrm{Al}$ & 1.65 & 1.19 & 1.17 & 2.78 & 2.84 & 2.77 & 0.00 & 0.00 \\
$\mathrm{Cr}$ & 0.00 & 0.00 & 0.00 & 0.00 & 0.00 & 0.00 & 0.00 & 0.00 \\
$\mathrm{Fe}$ & 1.36 & 0.00 & 0.00 & 0.05 & 2.38 & 2.56 & 0.99 & 0.99 \\
$\mathrm{Mn}$ & 0.00 & 0.00 & 0.00 & 0.00 & 0.00 & 0.00 & 0.01 & 0.01 \\
$\mathrm{Mg}$ & 0.97 & 0.00 & 0.00 & 0.06 & 2.05 & 1.94 & 0.00 & 0.01 \\
$\mathrm{Ca}$ & 0.00 & 0.18 & 0.17 & 0.00 & 0.00 & 0.00 & 0.00 & 0.00 \\
$\mathrm{Na}$ & 0.03 & 0.81 & 0.82 & 0.23 & 0.02 & 0.01 & 0.00 & 0.00 \\
$\mathrm{~K}$ & 0.85 & 0.00 & 0.00 & 0.72 & 0.01 & 0.01 & 0.00 & 0.00 \\
$\mathrm{Sum}$ & 7.74 & 5.00 & 5.00 & 6.96 & 9.94 & 9.96 & 2.01 & 2.01 \\
\hline $\mathrm{X}_{M g}$ & 0.42 & - & - & - & 0.46 & 0.43 & - & - \\
$\mathrm{X}_{N a}$ & - & - & - & 0.24 & - & - & - & - \\
$\mathrm{X}_{C a}$ & - & 0.18 & 0.17 & - & - & - & - & - \\
\hline & & & & & & & & \\
$\mathrm{Fa}$ & & & & & & & &
\end{tabular}

Representative mineral compositions in sample 24-99.

\section{Appendix 2.3 - Garnet compositional data}




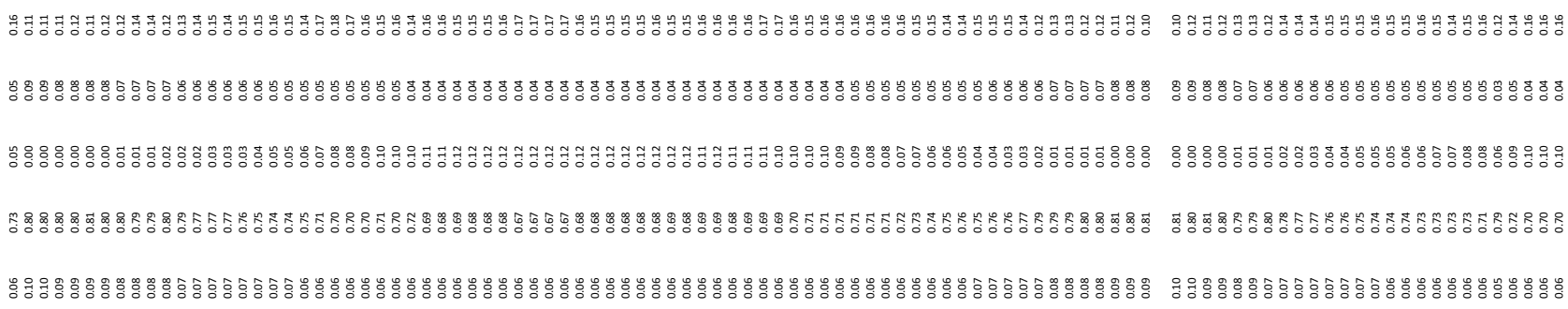




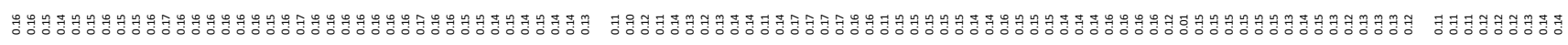

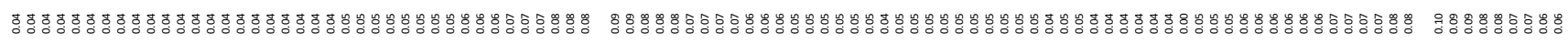

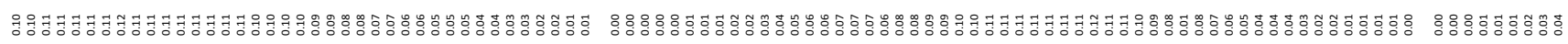

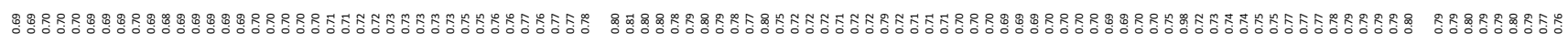

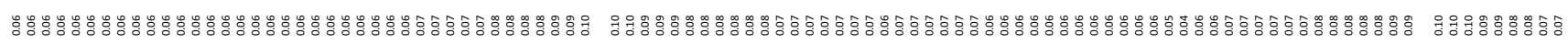

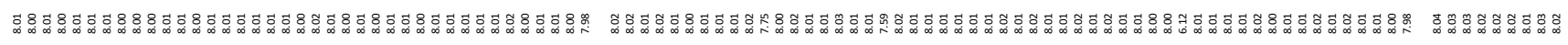

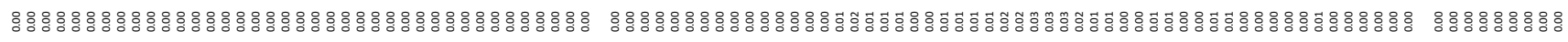

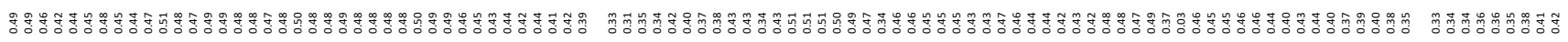

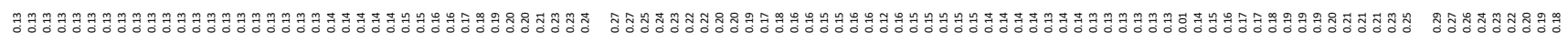

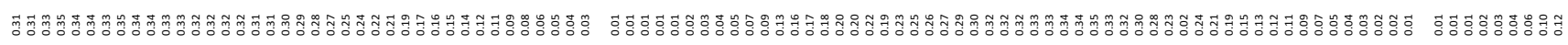

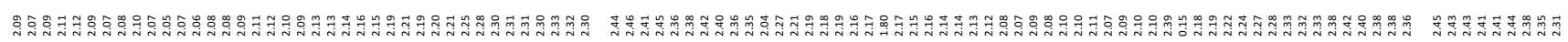

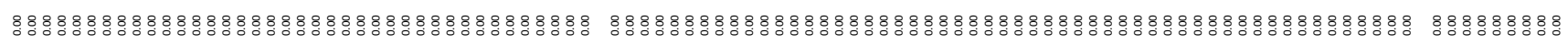

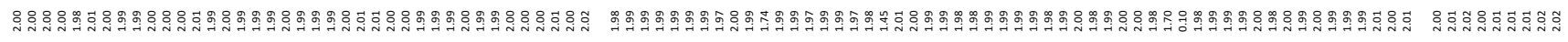

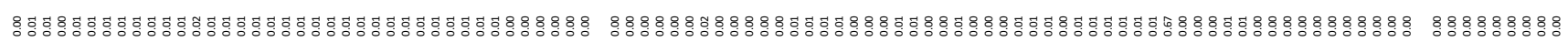

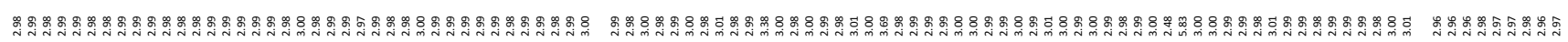

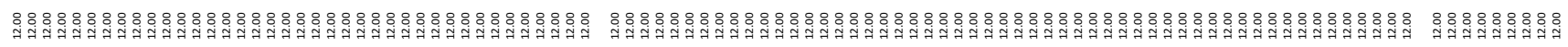

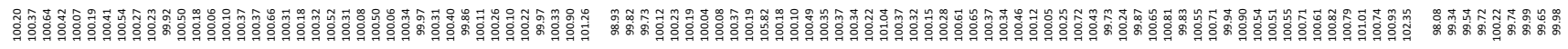

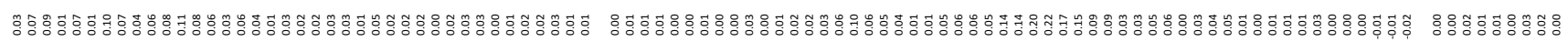

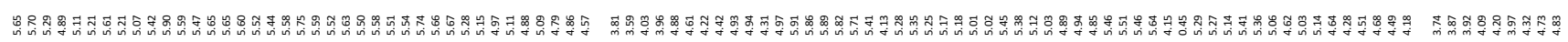

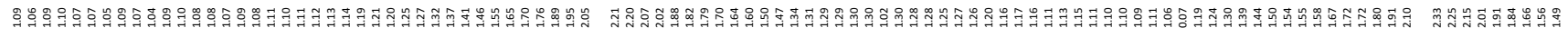

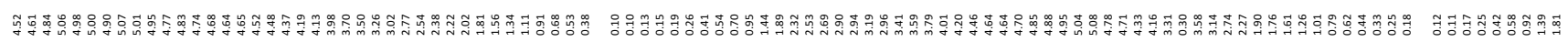

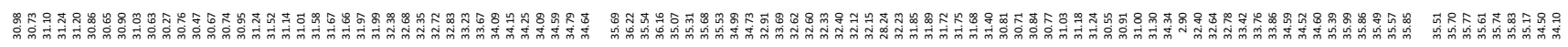

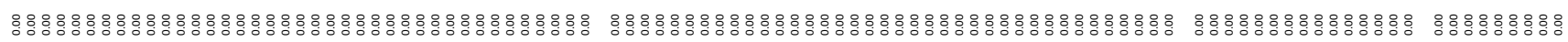

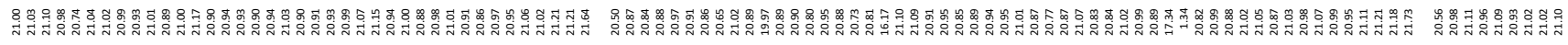

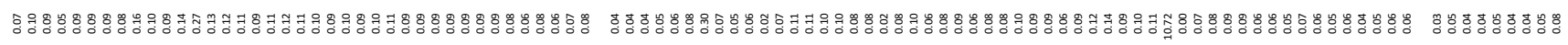

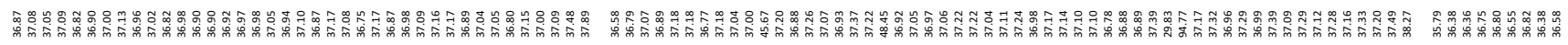

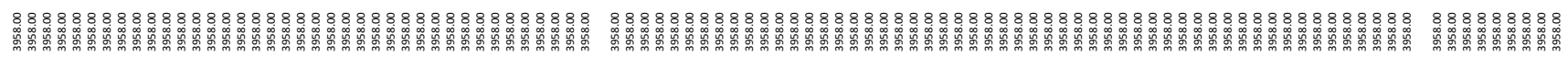

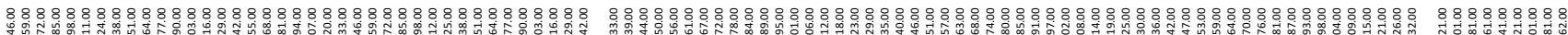

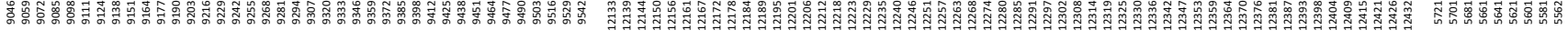

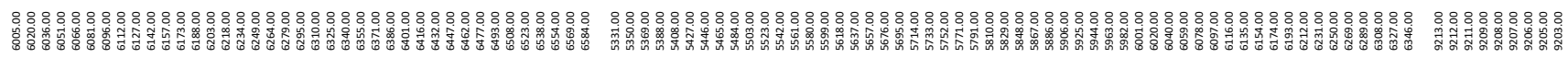
椟罢 罢

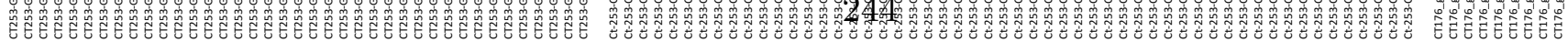

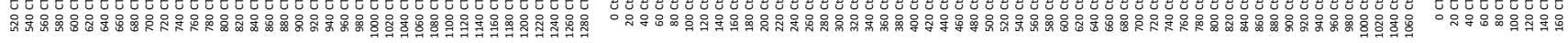
ล 


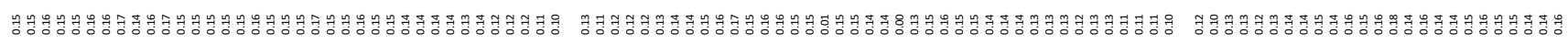

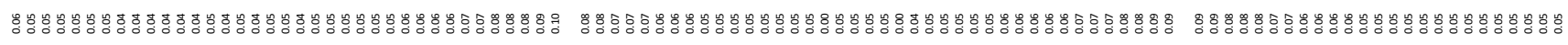

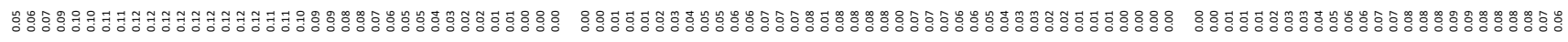

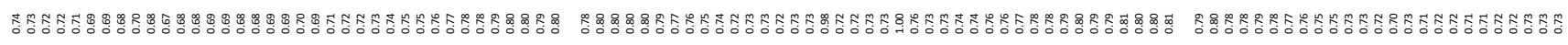

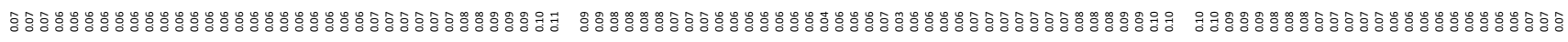

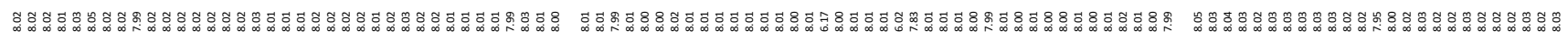

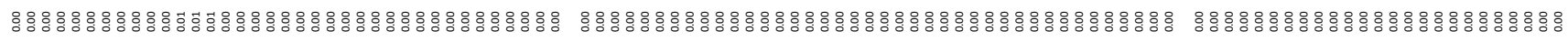

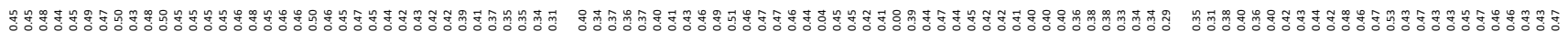

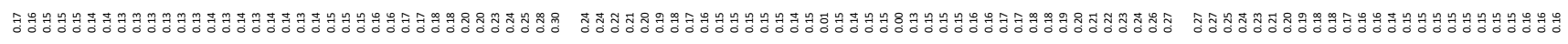

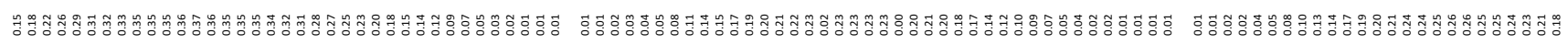

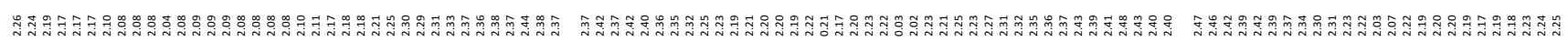

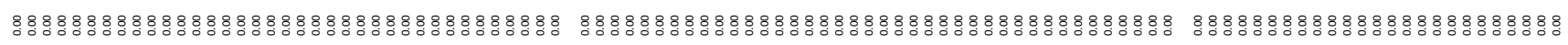

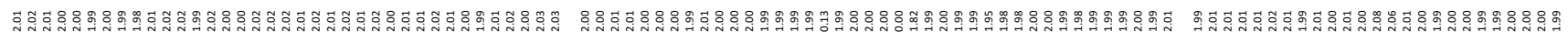

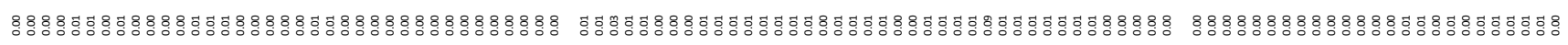

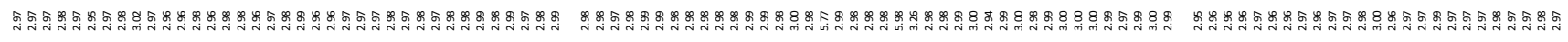
8.

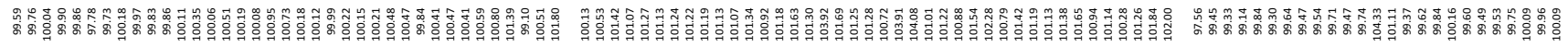

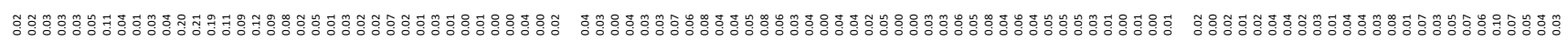

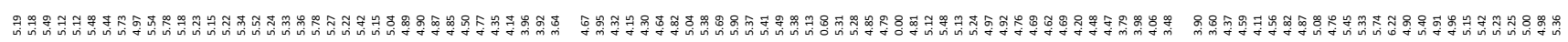

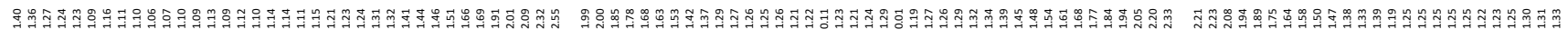

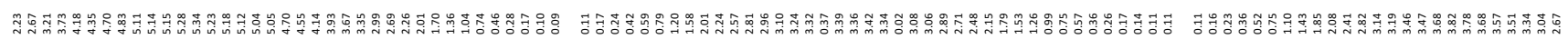

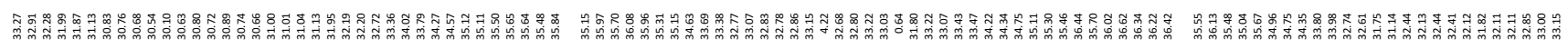

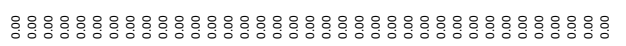

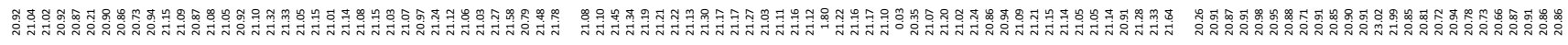

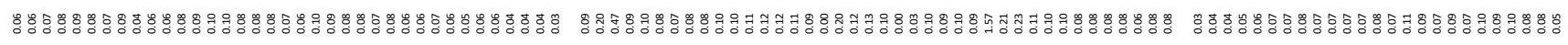

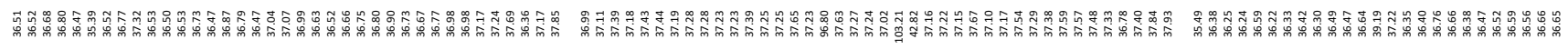

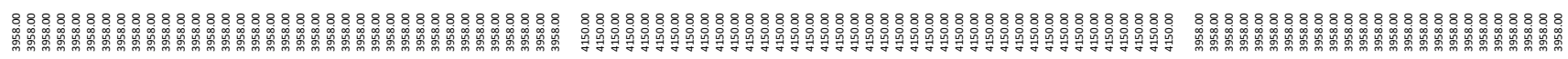

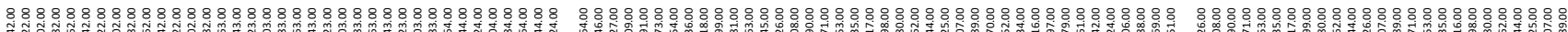

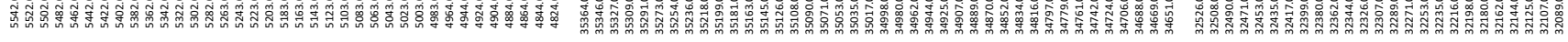

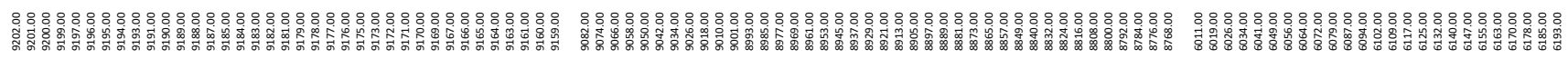

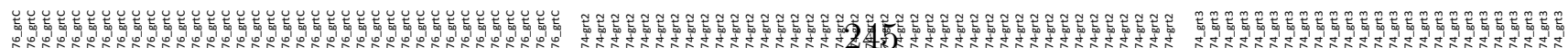

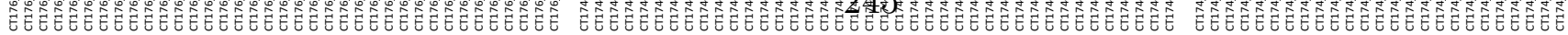

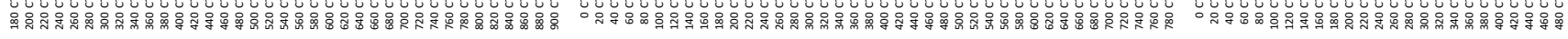




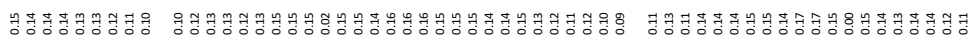

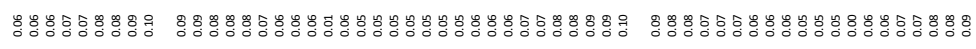

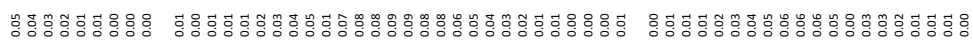

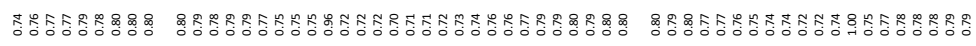

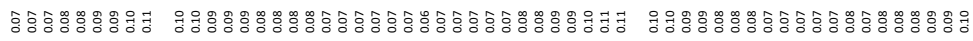

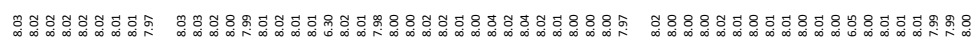

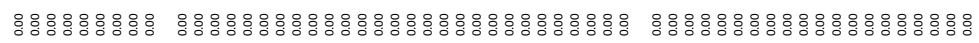

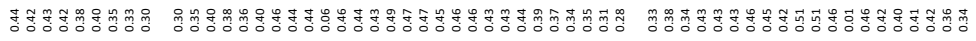

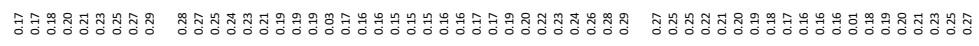

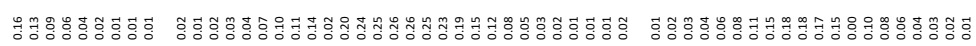

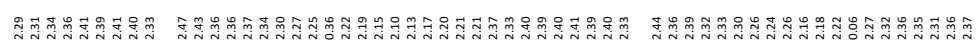
웅

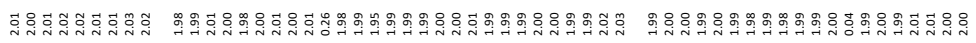

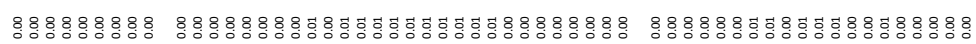

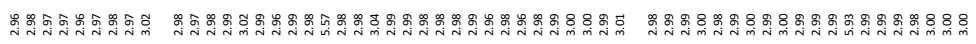
8.

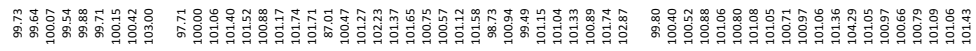

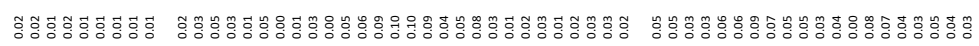

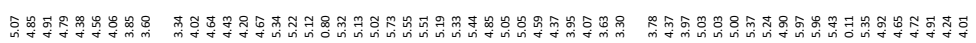

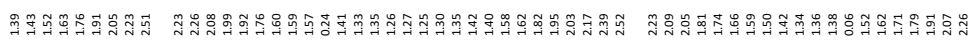

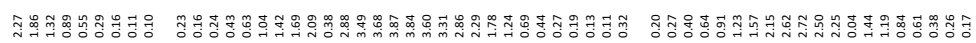

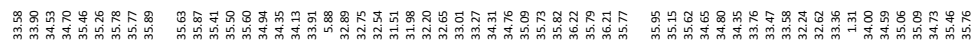

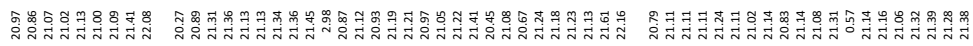

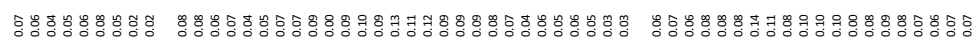

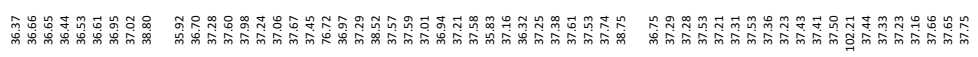

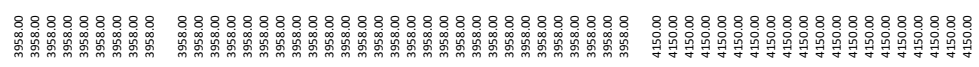
888888888888888888888888888888888888888888888888888

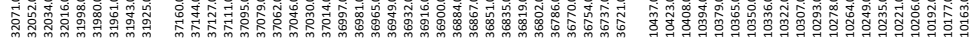

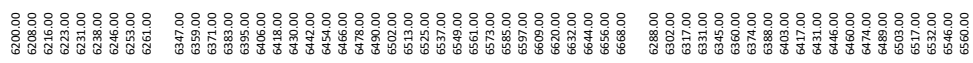

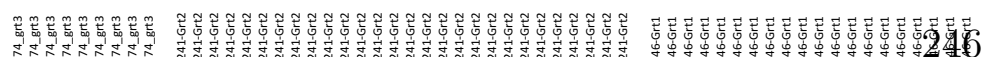

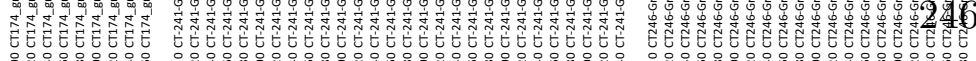

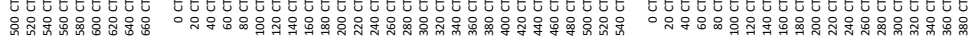

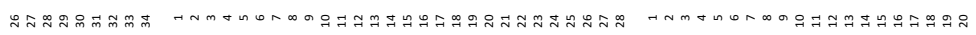




\section{Appendix 2.4 - Thermodynamic dataset and input files used with Theriak- Domino and THERIA_G}

\begin{tabular}{|c|c|c|c|c|c|c|c|}
\hline \multicolumn{8}{|l|}{25} \\
\hline 0 & $\mathrm{AL}$ & BA & C & $\mathrm{CA}$ & CL & $\mathrm{CO}$ & \\
\hline $\mathrm{CU}$ & $\mathrm{F}$ & $\mathrm{FE}$ & $\mathrm{H}$ & $\mathrm{K}$ & MN & MG & \\
\hline NA & NI & $\mathrm{P}$ & $\mathrm{S}$ & SI & $\mathrm{SR}$ & TI & \\
\hline ZN & $\mathrm{ZR}$ & B & $\mathrm{E}$ & & & & \\
\hline 15.99940 & 26.98154 & 137.32700 & 12.01 & 40.07800 & 35.45270 & 58.93320 & \\
\hline 63.54600 & 18.99840 & 55.84700 & 1.00 & 39.09830 & 54.93085 & 24.30500 & \\
\hline 22.98977 & 58.69000 & 30.97362 & 32.06 & 28.08550 & 87.62000 & 47.88000 & \\
\hline 65.39000 & 91.22400 & 10.81000 & 1.00 & 000 & & & \\
\hline 0.0 & 1.5 & 1.0 & 2.0 & 1.0 & 0.0 & 1.0 & \\
\hline 1.0 & 0.0 & 1.0 & 0.5 & 0.5 & 1.0 & 1.0 & \\
\hline 0.5 & 1.0 & 1.0 & 0.0 & 2.0 & 1.0 & 2.0 & \\
\hline 1.0 & 2.0 & 0.0 & 0.0 & & & & \\
\hline \multicolumn{2}{|c|}{$* * * *$ MINERAL DATA $* * * * *$} & from Ho & olland & and Powell (1 & 1998+) & & \\
\hline pyrope & & $\mathrm{SI}(3) \mathrm{AL}(2) \mathrm{M}$ & IG (3) 0 & (12) py & $3 \mathrm{nl}$ & & \\
\hline $\mathrm{ST}$ & 0.0 & -6284720 & .000 & 266.3000 & 11.3 & 3180 & \\
\hline C3 & 633.50000 & 0.0000 & 0000 & -5196100.0 & -4315 & 5.200 & 0.00 \\
\hline VHP & .000043600 & & 37.0 & 0.0000 & & 0.00 & 0.00000 \\
\hline VH2 & 10.0000 & & 0000 & -0.000260550 & & & \\
\hline almandine & & $\mathrm{SI}(3) \mathrm{AL}(2) \mathrm{F}$ & $\mathrm{FE}(3) 0$ & (12) $\quad$ alm & $3 n$ & & \\
\hline ST & 0.0 & -5263520 & .000 & 340.0000 & 11. & 5110 & \\
\hline $\mathrm{C} 3$ & 677.30000 & 0.0000 & 0000 & -3772700.0 & -5044 & .000 & 0.00 \\
\hline VHP & .000040300 & & 90.0 & 0.0000 & & 0.00 & 0.00000 \\
\hline VH2 & 10.0000 & & 0000 & -0.000253500 & & & \\
\hline spessartine & & $\mathrm{SI}(3) \mathrm{AL}(2) \mathrm{M}$ & $\operatorname{MN}(3) 0$ & (12) $\quad$ spss & $3 n$ & & \\
\hline ST & 0.0 & -5646400 & .000 & 367.0000 & 11. & 7920 & \\
\hline C3 & 584.60000 & -0.0015 & 5930 & -7516700.0 & -2750 & .100 & 0.00 \\
\hline VHP & .000046200 & 179 & 90.0 & 0.0000 & & 0.00 & 0.00000 \\
\hline VH2 & 10.0000 & & 0000 & -0.000268500 & & & \\
\hline grossular & & $\mathrm{SI}(3) \mathrm{AL}(2) \mathrm{C}$ & $\mathrm{CA}(3) \mathrm{O}$ & (12) $\mathrm{gr}$ & $3 n$ & & \\
\hline $\mathrm{ST}$ & 0.0 & -6644150 & .000 & 255.0000 & 12. & 5350 & \\
\hline C3 & 626.00000 & 0.0000 & 0000 & -5779200.0 & -4002 & 2.900 & 0.00 \\
\hline VHP & .000039300 & & 80.0 & 0.0000 & & 0.00 & 0.00000 \\
\hline VH2 & 10.0000 & & 0000 & -0.000252000 & & & \\
\hline andalusite & & $\mathrm{SI}(1) \mathrm{AL}(2) 0$ & $0(5)$ & and & $1 \mathrm{nh}$ & & \\
\hline ST & 0.0 & -2588800 & .000 & 92.7000 & & 1530 & \\
\hline C3 & 277.30000 & -0.0065 & 5880 & -1914100.0 & -2265 & .600 & 0.00 \\
\hline VHP & .000041100 & & 34.0 & 0.0000 & & 0.00 & 0.00000 \\
\hline VH2 & 10.0000 & & 0000 & -0.000200100 & & & \\
\hline kyanite & & $\mathrm{SI}(1) \mathrm{AL}(2) \mathrm{O}$ & $0(5)$ & ky & $1 \mathrm{nh}$ & & \\
\hline ST & 0.0 & -2593110 & .000 & 83.5000 & & 4140 & \\
\hline C3 & 279.40000 & -0.0071 & 1240 & -2055600.0 & -2289 & .400 & 0.00 \\
\hline VHP & .000040400 & 159 & 90.0 & 0.0000 & & 0.00 & 0.00000 \\
\hline VH2 & 10.0000 & & 0000 & -0.000238500 & & & \\
\hline sillimanite & & $\mathrm{SI}(1) \mathrm{AL}(2) 0$ & $0(5)$ & sill & $1 \mathrm{nh}$ & & \\
\hline $\mathrm{ST}$ & 0.0 & -2585680 & .000 & 95.5000 & & 9860 & \\
\hline C3 & 280.20000 & -0.0069 & 9000 & -1375700.0 & -2399 & .400 & 0.00 \\
\hline VHP & .000022100 & & 20.0 & 2200.0000 & & 4.00 & 0.03500 \\
\hline vH2 & 10.0000 & & 0000 & -0.000198000 & & & \\
\hline Mg-stauroli & Lite & $\mathrm{SI}(7.5) \mathrm{AL}(1$ & 18) MG ( & 4) $\mathrm{O}(48) \mathrm{H}(4)$ & mst & $4 \mathrm{nh}$ & \\
\hline ST & 0.0 & -25101490 & .000 & 910.0000 & 44. & 2600 & \\
\hline C3 & 2820.50000 & -0.0593 & 3660 & -13774000.0 & -24126 & .000 & 0.00 \\
\hline VHP & .000012000 & & 00.0 & 0.0000 & & 0.00 & 0.00000 \\
\hline VH2 & 10.0000 & & 0000 & -0.000180000 & & & \\
\hline Fe-stauroli & Lite & $\mathrm{SI}(7.5) \mathrm{AL}$ & 18) $\mathrm{FE}$ & 4) $\mathrm{O}(48) \mathrm{H}(4)$ & fst & $4 \mathrm{nh}$ & \\
\hline ST & 0.0 & -23753930 & .000 & 1010.0000 & 44. & 8800 & \\
\hline C3 & 2880.00000 & -0.0565 & 5950 & -10642000.0 & -25373 & 3.000 & 0.00 \\
\hline VHP & .000012000 & & 00.0 & 0.0000 & & 0.00 & 0.00000 \\
\hline VH2 & 10.0000 & & 0000 & -0.000180000 & & & \\
\hline Mn-stauroli & Lite & $\mathrm{SI}(7.5) \mathrm{AL}$ & 18) $\mathrm{MN}$ & 4) $\mathrm{O}(48) \mathrm{H}(4)$ & mnst & $4 \mathrm{nh}$ & \\
\hline ST & 0.0 & -24203880 & .000 & 1024.0000 & 45. & 4600 & \\
\hline C3 & 2873.30000 & -0.0890 & 0640 & -12688000.0 & -24749 & .000 & 0.00 \\
\hline
\end{tabular}




\begin{tabular}{|c|c|c|c|c|c|}
\hline \multirow{2}{*}{$\begin{array}{l}\text { VHP } \\
\text { VH2 }\end{array}$} & 0.000012000 & 1200.0 & 0.0000 & \multirow[t]{2}{*}{0.00} & \multirow[t]{2}{*}{0.00000} \\
\hline & 10.0000 & 4.0000 & -0.000180000 & & \\
\hline \multicolumn{2}{|c|}{ Mg-chloritoid } & \multicolumn{2}{|c|}{$\mathrm{SI}$ (1) AL (2)MG (1) O(7)H(2) mctd } & \multirow{2}{*}{$1 \mathrm{nh}$} & \\
\hline ST & 0.0 & -3551420.000 & 140.0000 & & \\
\hline $\mathrm{C} 3$ & 464.40000 & -0.0126540 & -1147200.0 & -4341.000 & 0.00 \\
\hline VHP & 0.000054200 & 1465.0 & 0.0000 & 0.00 & 0.00000 \\
\hline VH2 & 10.0000 & 4.0000 & -0.000219750 & & \\
\hline $\mathrm{Fe}-\mathrm{ch}$ & itoid & $\mathrm{SI}$ (1) AL (2) FE (1) & $(7) \mathrm{H}(2) \quad$ fctd & $1 \mathrm{nh}$ & \\
\hline ST & 0.0 & -3215380.000 & 155.0000 & 6.9800 & \\
\hline $\mathrm{C} 3$ & 484.60000 & -0.0138080 & -198900.0 & -4762.200 & 0.00 \\
\hline VHP & 0.000054200 & 1465.0 & 0.0000 & 0.00 & 0.00000 \\
\hline VH2 & 10.0000 & 4.0000 & -0.000219750 & & \\
\hline $\mathrm{Mn}-\mathrm{ch}$ & itoid & $\mathrm{SI}$ (1) AL (2) MN (1) & (7) $\mathrm{H}(2) \quad$ mnctd & $1 \mathrm{nh}$ & \\
\hline ST & 0.0 & -3329280.000 & 166.0000 & 7.1750 & \\
\hline $\mathrm{C} 3$ & 464.40000 & -0.0126540 & -1147200.0 & -4341.000 & 0.00 \\
\hline VHP & 0.000054200 & 1465.0 & 0.0000 & 0.00 & 0.00000 \\
\hline VH2 & 10.0000 & 4.0000 & -0.000219750 & & \\
\hline !zois & & $\mathrm{SI}(3) \mathrm{AL}(3) \mathrm{CA}(2$ & $\mathrm{O}(13) \mathrm{H}(1) \quad \mathrm{zo}$ & $1 \mathrm{nh}$ & \\
\hline ! ST & 0.0 & -6898610.000 & 297.0000 & 13.5750 & \\
\hline ! C3 & 595.70000 & 0.0622970 & -5921300.0 & -3394.700 & 0.00 \\
\hline ! VHP & 0.000067000 & 1120.0 & 0.0000 & 0.00 & 0.00000 \\
\hline ! VH2 & 10.0000 & 4.0000 & -0.000168000 & & \\
\hline clino & site & $\mathrm{SI}(3) \mathrm{AL}$ (3) CA (2) & (13) $\mathrm{H}(1) \quad \mathrm{czO}$ & $1 \mathrm{nh}$ & \\
\hline $\mathrm{ST}$ & 0.0 & -6898150.000 & 301.0000 & 13.6300 & \\
\hline $\mathrm{C} 3$ & 567.00000 & 0.0180630 & -7034000.0 & -2603.000 & 0.00 \\
\hline VHP & 0.000046000 & 1120.0 & 0.0000 & 0.00 & 0.00000 \\
\hline VH2 & 10.0000 & 4.0000 & -0.000168000 & & \\
\hline $\mathrm{Fe}-\mathrm{ep}$ & & $\mathrm{SI}$ (3) AL (1) FE (2) & $A(2) O(13) H(1)$ & fep & $2 \mathrm{nh}$ \\
\hline $\mathrm{ST}$ & 0.0 & -6002180.000 & 357.0000 & 14.1900 & \\
\hline $\mathrm{C} 3$ & 520.10000 & 0.0314990 & -15426000.0 & 218.800 & 0.00 \\
\hline VHP & 0.000050500 & 1294.0 & 0.0000 & 0.00 & 0.00000 \\
\hline VH2 & 10.0000 & 4.0000 & -0.000199280 & & \\
\hline epido & & $\mathrm{SI}(3) \mathrm{AL}$ (2) FE (1) & $A(2) O(13) H(1)$ & ep & $2 \mathrm{nh}$ \\
\hline ST & 0.0 & -6463210.000 & 328.0000 & 13.9100 & \\
\hline C3 & 544.60000 & 0.0247810 & -11230000.0 & -1192.100 & 0.00 \\
\hline VHP & 0.000050500 & 1233.0 & 0.0000 & 0.00 & 0.00000 \\
\hline VH2 & 10.0000 & 4.0000 & -0.000184950 & & \\
\hline cordi & & SI (5) AL (4) MG (2) & (18) $\quad$ crd & $1 \mathrm{nh}$ & \\
\hline ST & 0.0 & -9163370.000 & 407.5000 & 23.3220 & \\
\hline $\mathrm{C} 3$ & 821.30000 & 0.0433390 & -8211200.0 & -5000.000 & 0.00 \\
\hline VHP & 0.000007600 & 810.0 & 1800.0000 & 20.00 & 0.20000 \\
\hline VH2 & 10.0000 & 4.0000 & -0.000121500 & & \\
\hline $\mathrm{Fe}-\mathrm{Co}$ & erite & $\mathrm{SI}(5) \mathrm{AL}(4) \mathrm{FE}(2)$ & (18) fcrd & $2 \mathrm{nh}$ & \\
\hline $\mathrm{ST}$ & 0.0 & -8436070.000 & 475.0000 & 23.7100 & \\
\hline $\mathrm{C} 3$ & 851.50000 & 0.0447240 & -6645000.0 & -5623.400 & 0.00 \\
\hline VHP & 0.000007600 & 810.0 & 1800.0000 & 20.00 & 0.20000 \\
\hline VH2 & 10.0000 & 4.0000 & -0.000121500 & & \\
\hline $\mathrm{Mn}-\mathrm{col}$ & erite & $\mathrm{SI}(5) \mathrm{AL}(4) \mathrm{MN}(2)$ & (18) mncrd & $1 \mathrm{nh}$ & \\
\hline $\mathrm{ST}$ & 0.0 & -8681180.000 & 475.0000 & 24.0270 & \\
\hline $\mathrm{C} 3$ & 847.70000 & 0.0284900 & -7668200.0 & -5311.400 & 0.00 \\
\hline VHP & 0.000007600 & 810.0 & 1800.0000 & 20.00 & 0.20000 \\
\hline VH2 & 10.0000 & 4.0000 & -0.000121500 & & \\
\hline phase & & $\mathrm{SI}(2) \mathrm{MG}(7) 0(14)$ & (6) $\quad \mathrm{phA}$ & $7 \mathrm{nh}$ & \\
\hline $\mathrm{ST}$ & 0.0 & -7130410.000 & 350.0000 & 15.4420 & \\
\hline $\mathrm{C} 3$ & 964.00000 & -0.0115210 & -4517800.0 & -7724.700 & 0.00 \\
\hline VHP & 0.000082600 & 1450.0 & 0.0000 & 0.00 & 0.00000 \\
\hline VH2 & 10.0000 & 4.0000 & -0.000217500 & & \\
\hline $\mathrm{Mg}-\mathrm{ca}$ & olite & $\mathrm{SI}(2) \mathrm{AL}(2) \mathrm{MG}(1)$ & (10) $\mathrm{H}(4) \quad$ mcar & $1 \mathrm{nh}$ & \\
\hline ST & 0.0 & -4781240.000 & 210.0000 & 10.5900 & \\
\hline C3 & 667.80000 & -0.0125590 & -1167100.0 & -6440.000 & 0.00 \\
\hline VHP & 0.000050000 & 525.0 & 0.0000 & 0.00 & 0.00000 \\
\hline VH2 & 10.0000 & 4.0000 & -0.000078750 & & \\
\hline $\mathrm{Fe}-\mathrm{ca}$ & olite & $\mathrm{SI}(2) \mathrm{AL}(2) \mathrm{FE}(1)$ & (10) $\mathrm{H}(4) \quad$ fcar & $1 \mathrm{nh}$ & \\
\hline $\mathrm{ST}$ & 0.0 & -4413200.000 & 255.0000 & 10.6900 & \\
\hline $\mathrm{C} 3$ & 674.80000 & -0.0100920 & -715800.0 & -6554.500 & 0.00 \\
\hline VHP & 0.000050000 & 525.0 & 0.0000 & 0.00 & 0.00000 \\
\hline VH2 & 10.0000 & 4.0000 & -0.000078750 & & \\
\hline musco & & $\mathrm{SI}$ (3) AL (3)K (1) 0 & 12) $\mathrm{H}(2) \quad \mathrm{mu}$ & $2 \mathrm{nh}$ & \\
\hline
\end{tabular}




\begin{tabular}{|c|c|c|c|c|c|c|}
\hline ST & 0.0 & -5984180.000 & 292.0000 & 14.0830 & & \\
\hline C3 & 756.40000 & -0.0198400 & -2170000.0 & -6979.200 & & 0.00 \\
\hline VHP & 0.000059600 & 490.0 & 0.0000 & 0.00 & & 0.00000 \\
\hline VH2 & 10.0000 & 4.0000 & -0.000073500 & & & \\
\hline \multicolumn{2}{|c|}{ celadonite } & \multicolumn{2}{|c|}{$\mathrm{SI}(4) \mathrm{AL}(1) \mathrm{MG}(1) \mathrm{K}(1) \mathrm{O}(12) \mathrm{H}(2)$} & cel & \multicolumn{2}{|l|}{$1 \mathrm{nh}$} \\
\hline ST & 0.0 & -5842420.000 & 290.0000 & 13.9570 & & \\
\hline $\mathrm{C} 3$ & 741.20000 & -0.0187480 & -2368800.0 & -6616.900 & & 0.00 \\
\hline VHP & 0.000059600 & 700.0 & 0.0000 & 0.00 & & 0.00000 \\
\hline VH2 & 10.0000 & 4.0000 & -0.000105000 & & & \\
\hline \multicolumn{2}{|c|}{ Fe-celadonite } & \multicolumn{2}{|c|}{$\mathrm{SI}(4) \mathrm{AL}(1) \mathrm{FE}(1) \mathrm{K}(1) \mathrm{O}(12) \mathrm{H}(2)$} & fcel & \multicolumn{2}{|l|}{$1 \mathrm{nh}$} \\
\hline ST & 0.0 & -5477430.000 & 329.0000 & 14.0700 & & \\
\hline C3 & 756.30000 & -0.0191470 & -1586100.0 & -6928.700 & & 0.00 \\
\hline VHP & 0.000059600 & 700.0 & 0.0000 & 0.00 & & 0.00000 \\
\hline VH2 & 10.0000 & 4.0000 & -0.000105000 & & & \\
\hline \multicolumn{2}{|c|}{ paragonite } & \multicolumn{2}{|c|}{$\mathrm{SI}(3) \mathrm{AL}(3) \mathrm{NA}(1) \mathrm{O}(12) \mathrm{H}(2)$ pa } & \multicolumn{3}{|l|}{ 1nh } \\
\hline $\mathrm{ST}$ & 0.0 & -5946340.000 & 276.0000 & 13.2110 & & \\
\hline $\mathrm{C} 3$ & 803.00000 & -0.0315800 & 217000.0 & -8151.000 & & 0.00 \\
\hline VHP & 0.000077400 & 550.0 & 0.0000 & 0.00 & & 0.00000 \\
\hline VH2 & 10.0000 & 4.0000 & -0.000082500 & & & \\
\hline \multicolumn{2}{|c|}{ margarite } & \multicolumn{2}{|c|}{$\mathrm{SI}$ (2) AL (4) CA (1) O(12) H (2) $\mathrm{mrg}$} & \multicolumn{3}{|c|}{$1 \mathrm{nh}$} \\
\hline $\mathrm{ST}$ & 0.0 & -6241230.000 & 267.0000 & 12.9640 & & \\
\hline C3 & 744.40000 & -0.0168000 & -2074400.0 & -6783.200 & & 0.00 \\
\hline VHP & 0.000048700 & 1300.0 & 0.0000 & 0.00 & & 0.00000 \\
\hline VH2 & 10.0000 & 4.0000 & -0.000195000 & & & \\
\hline phlog & & $\mathrm{SI}$ (3) AL (1) MG (3) & $(1) \mathrm{O}(12) \mathrm{H}(2)$ & phl & $2 \mathrm{nh}$ & \\
\hline $\mathrm{ST}$ & 0.0 & -6219160.000 & 328.0000 & 14.9640 & & \\
\hline C3 & 770.30000 & -0.0369390 & -2328900.0 & -6531.600 & & 0.00 \\
\hline VHP & 0.000057900 & 513.0 & 0.0000 & 0.00 & & 0.00000 \\
\hline VH2 & 10.0000 & 4.0000 & -0.000076950 & & & \\
\hline annit & & $\mathrm{SI}$ (3) AL (1) FE (3) & (1) $\mathrm{O}(12) \mathrm{H}(2)$ & ann & $3 n h$ & \\
\hline $\mathrm{ST}$ & 0.0 & -5151670.000 & 418.0000 & 15.4320 & & \\
\hline $\mathrm{C} 3$ & 815.70000 & -0.0348610 & 19800.0 & -7466.700 & & 0.00 \\
\hline VHP & 0.000057900 & 513.0 & 0.0000 & 0.00 & & 0.00000 \\
\hline VH2 & 10.0000 & 4.0000 & -0.000076950 & & & \\
\hline $\mathrm{Mn}-\mathrm{bi}$ & & $\mathrm{SI}$ (3) AL (1) MN (3) & (1) $\mathrm{O}(12) \mathrm{H}(2)$ & mnbi & $2 \mathrm{nh}$ & \\
\hline ST & 0.0 & -5462840.000 & 433.0000 & 15.2640 & & \\
\hline C3 & 809.90000 & -0.0592130 & -1514400.0 & -6998.700 & & 0.00 \\
\hline VHP & 0.000057900 & 513.0 & 0.0000 & 0.00 & & 0.00000 \\
\hline VH2 & 10.0000 & 4.0000 & -0.000076950 & & & \\
\hline eastol & & $\mathrm{SI}(2) \mathrm{AL}$ (3) MG (2) & (1) $\mathrm{O}(12) \mathrm{H}(2)$ & east & $2 \mathrm{nh}$ & \\
\hline $\mathrm{ST}$ & 0.0 & -6338170.000 & 318.0000 & 14.7380 & & \\
\hline C3 & 785.50000 & -0.0380310 & -2130300.0 & -6893.700 & & 0.00 \\
\hline VHP & 0.000057900 & 513.0 & 0.0000 & 0.00 & & 0.00000 \\
\hline VH2 & 10.0000 & 4.0000 & -0.000076950 & & & \\
\hline $\mathrm{Na}-\mathrm{ph}$ & opite & $\mathrm{SI}$ (3) AL (1) MG (3) I & $A(1) O(12) H(2)$ & naph & $1 \mathrm{nh}$ & \\
\hline ST & 0.0 & -6172820.000 & 318.0000 & 14.4500 & & \\
\hline $\mathrm{C} 3$ & 773.50000 & -0.0402290 & -2597900.0 & -6512.600 & & 0.00 \\
\hline VHP & 0.000057900 & 513.0 & 0.0000 & 0.00 & & 0.00000 \\
\hline VH2 & 10.0000 & 4.0000 & -0.000076950 & & & \\
\hline clino & ore & $\mathrm{SI}$ (3) AL (2) MG (5) & $(18) \mathrm{H}(8) \quad$ clin & $2 \mathrm{nh}$ & & \\
\hline $\mathrm{ST}$ & 0.0 & -8912410.000 & 430.5000 & 21.0900 & & \\
\hline C3 & 1161.80000 & 0.0101330 & -7657300.0 & -9690.900 & & 0.00 \\
\hline VHP & 0.000039800 & 870.0 & 0.0000 & 0.00 & & 0.00000 \\
\hline VH2 & 10.0000 & 4.0000 & -0.000130500 & & & \\
\hline amesi & & $\mathrm{SI}$ (2) AL (4)MG (4) & (18) H(8) ames & $2 \mathrm{nh}$ & & \\
\hline $\mathrm{ST}$ & 0.0 & -9034440.000 & 410.0000 & 20.5200 & & \\
\hline $\mathrm{C} 3$ & 1177.00000 & 0.0090410 & -7458700.0 & -10053.000 & & 0.00 \\
\hline VHP & 0.000039800 & 870.0 & 0.0000 & 0.00 & & 0.00000 \\
\hline VH2 & 10.0000 & 4.0000 & -0.000130500 & & & \\
\hline ! Al-f & -chlorite & $\mathrm{SI}(4) \mathrm{MG}(6) \mathrm{O}(18)$ & $\mathrm{H}(8) \quad$ af chl & $2 \mathrm{nh}$ & & \\
\hline ! ST & 0.0 & -8727380.000 & 428.0000 & 21.6600 & & \\
\hline ! C3 & 1146.60000 & 0.0112250 & -7855900.0 & -9328.800 & & 0.00 \\
\hline ! VHP & 0.000039800 & 870.0 & 0.0000 & 0.00 & & 0.00000 \\
\hline ! VH2 & 10.0000 & 4.0000 & -0.000130500 & & & \\
\hline daphn & & $\mathrm{SI}(3) \mathrm{AL}(2) \mathrm{FE}(5)$ & $(18) \mathrm{H}(8) \quad$ daph & $3 n h$ & & \\
\hline ST & 0.0 & -7134850.000 & 565.0000 & 21.3400 & & \\
\hline C3 & 1237.40000 & 0.0135940 & -3743000.0 & -11250.000 & & 0.00 \\
\hline VHP & 0.000039800 & 870.0 & 0.0000 & 0.00 & & 0.00000 \\
\hline
\end{tabular}




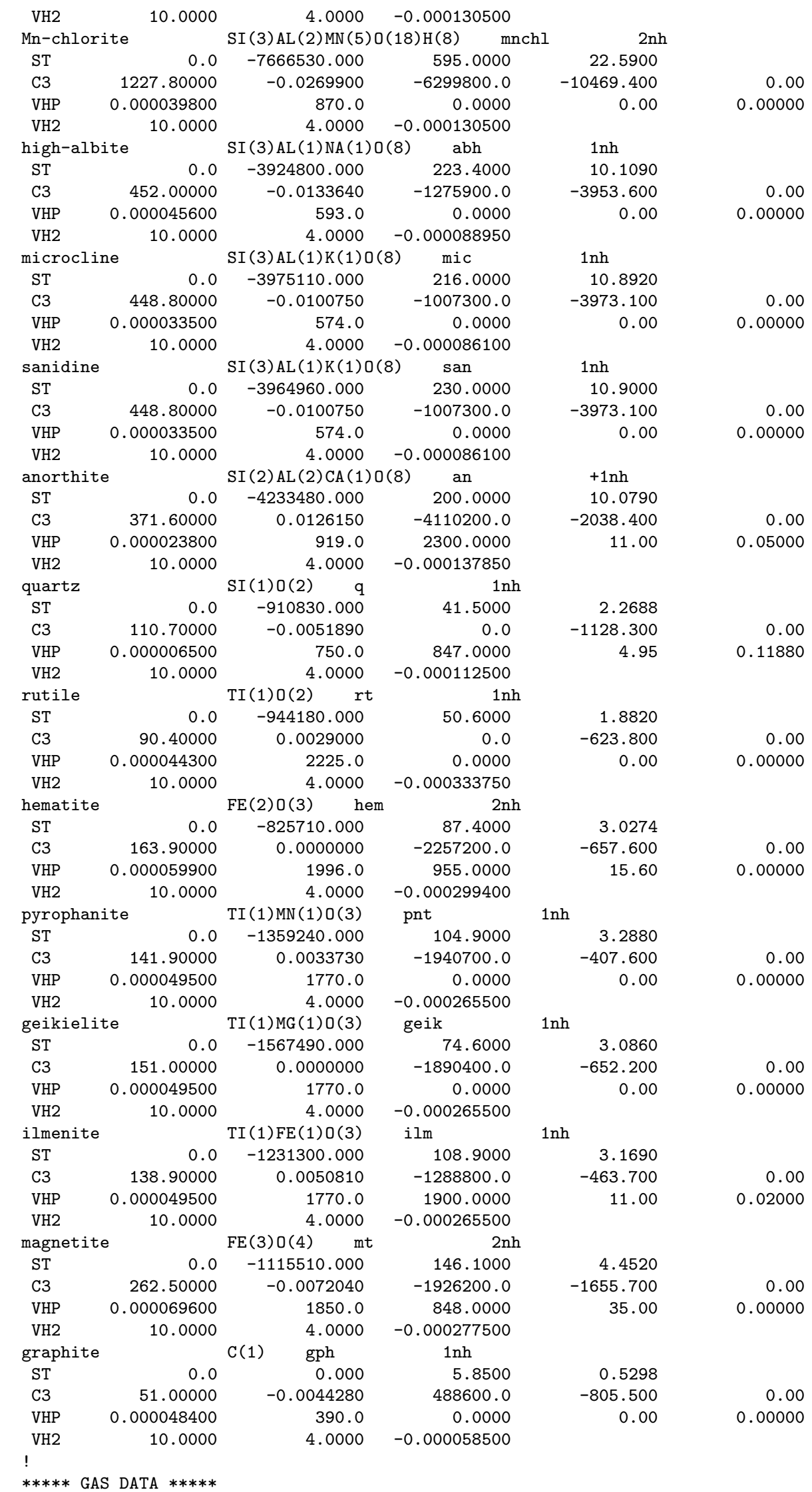




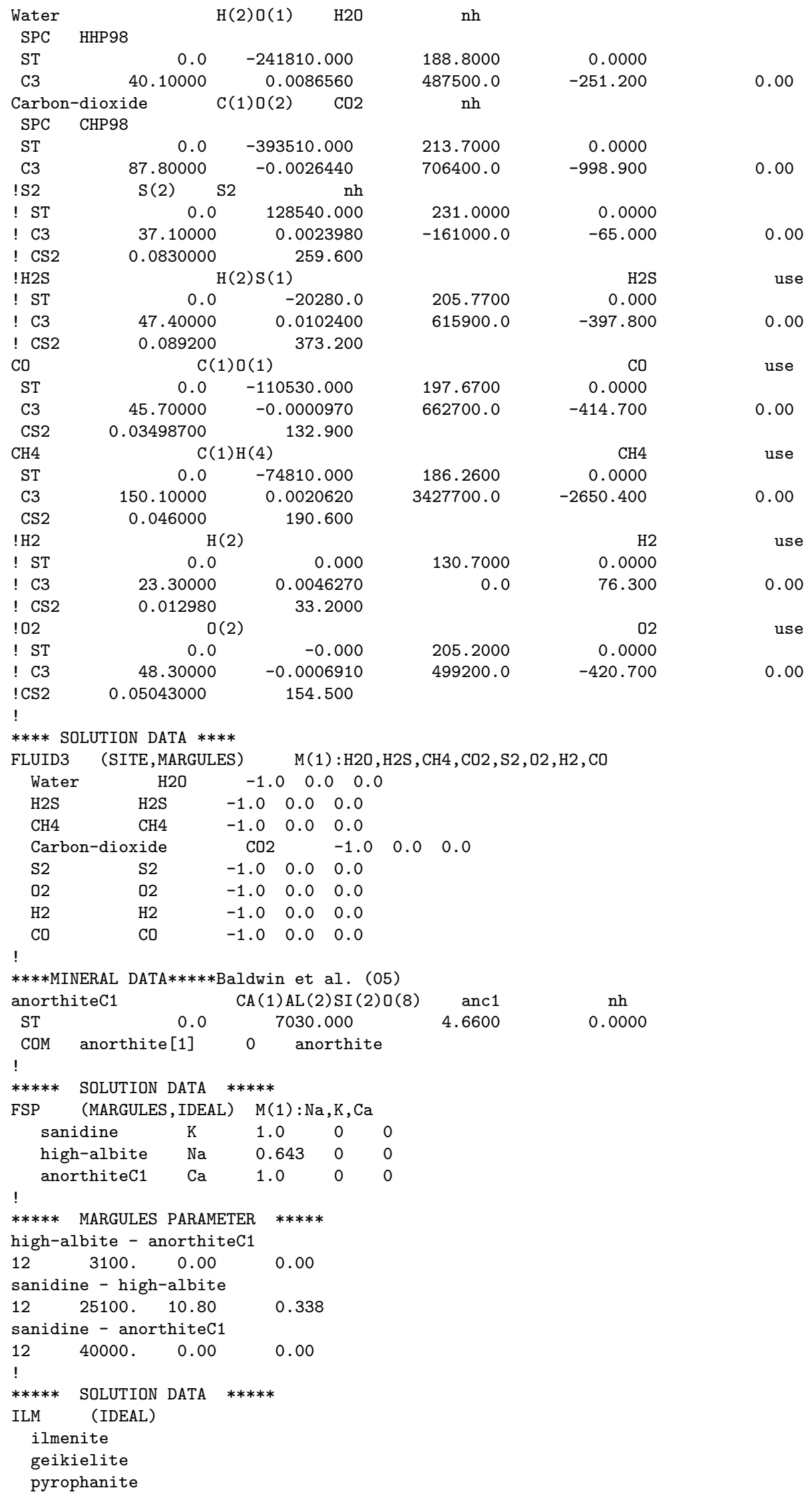




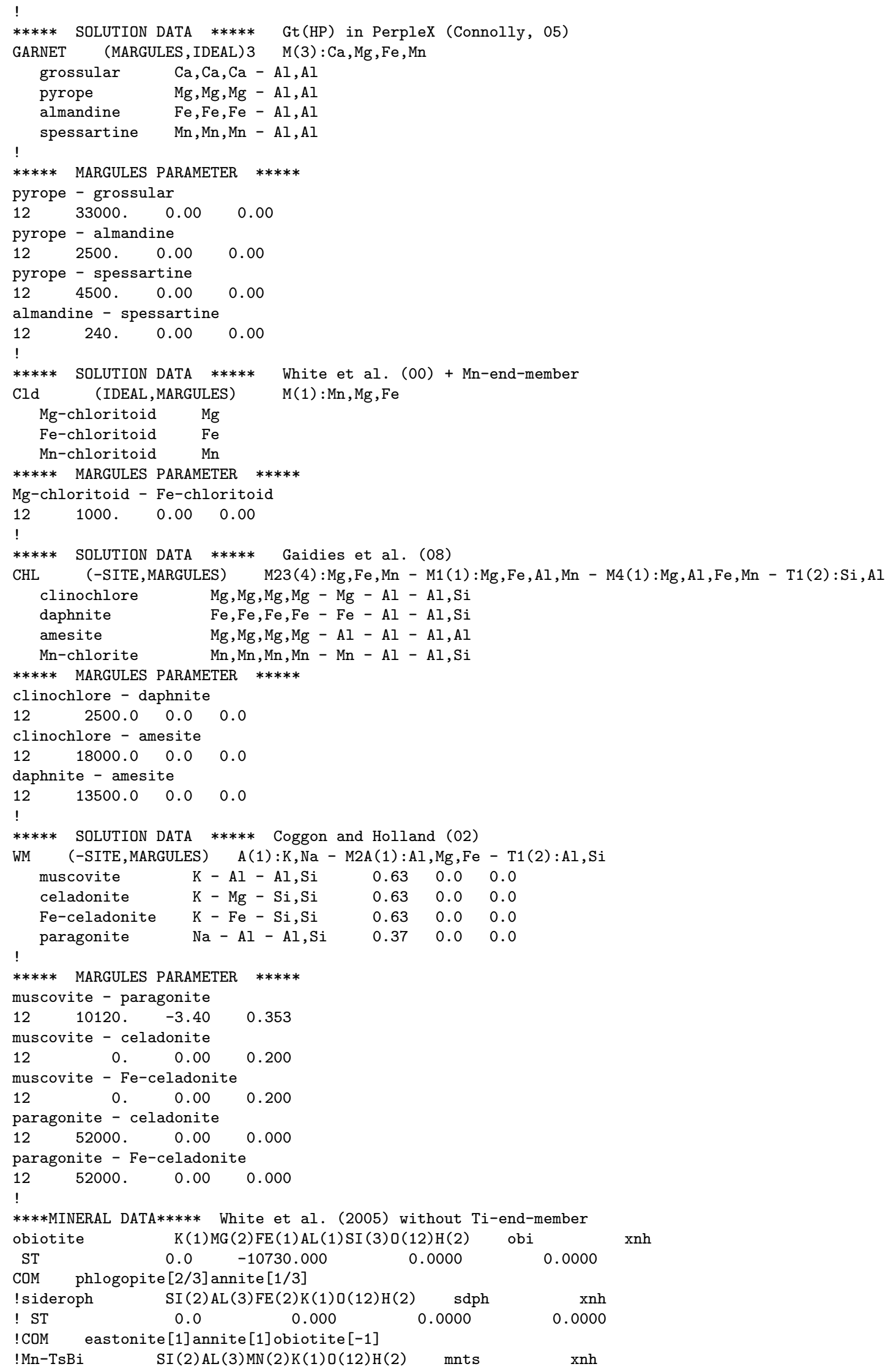




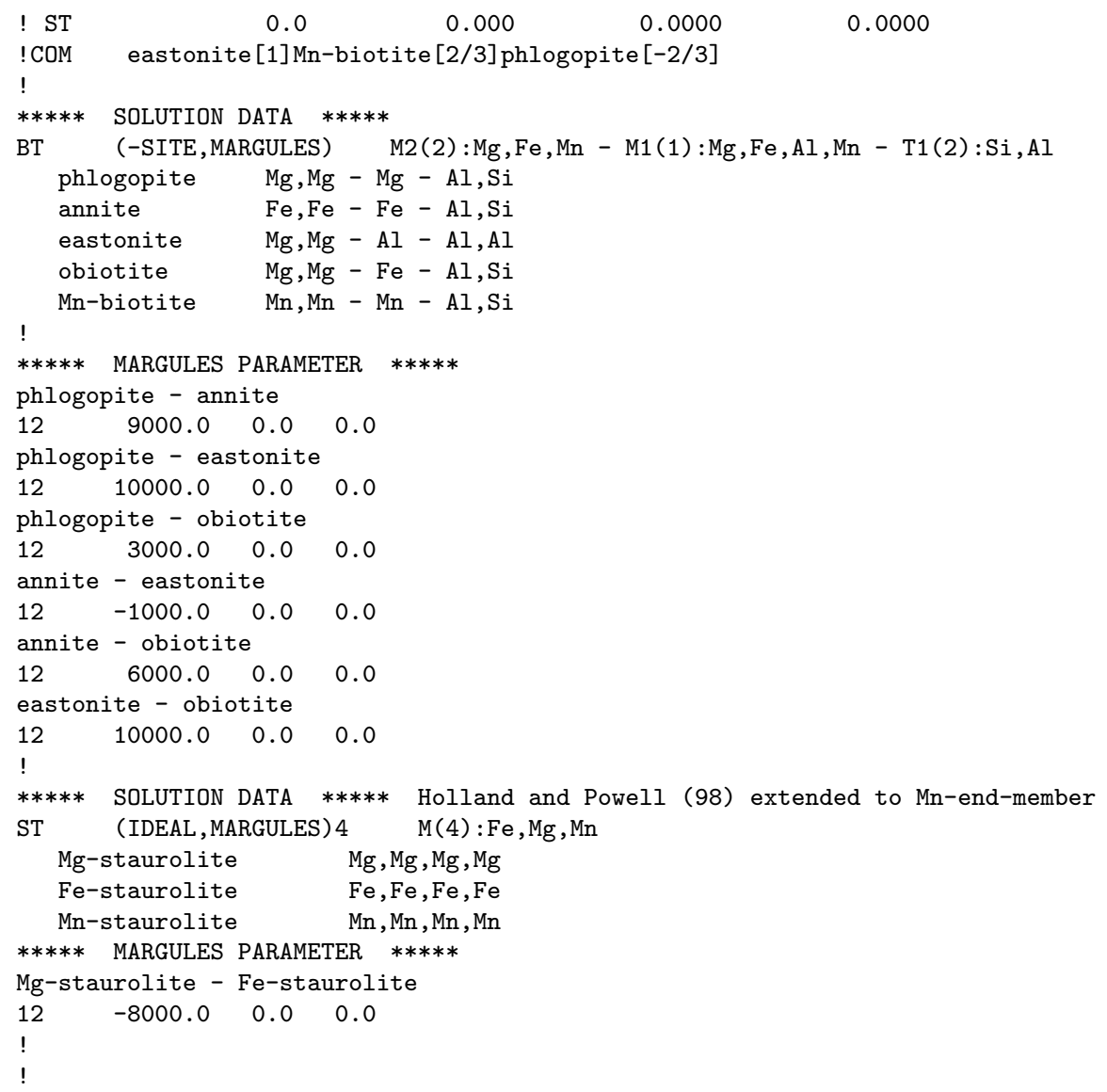




\section{Appendix 2.5 - Input files used for THERIA_G modelling of garnet crystalliza- tion in 24-99}

theriag_CSD.txt:

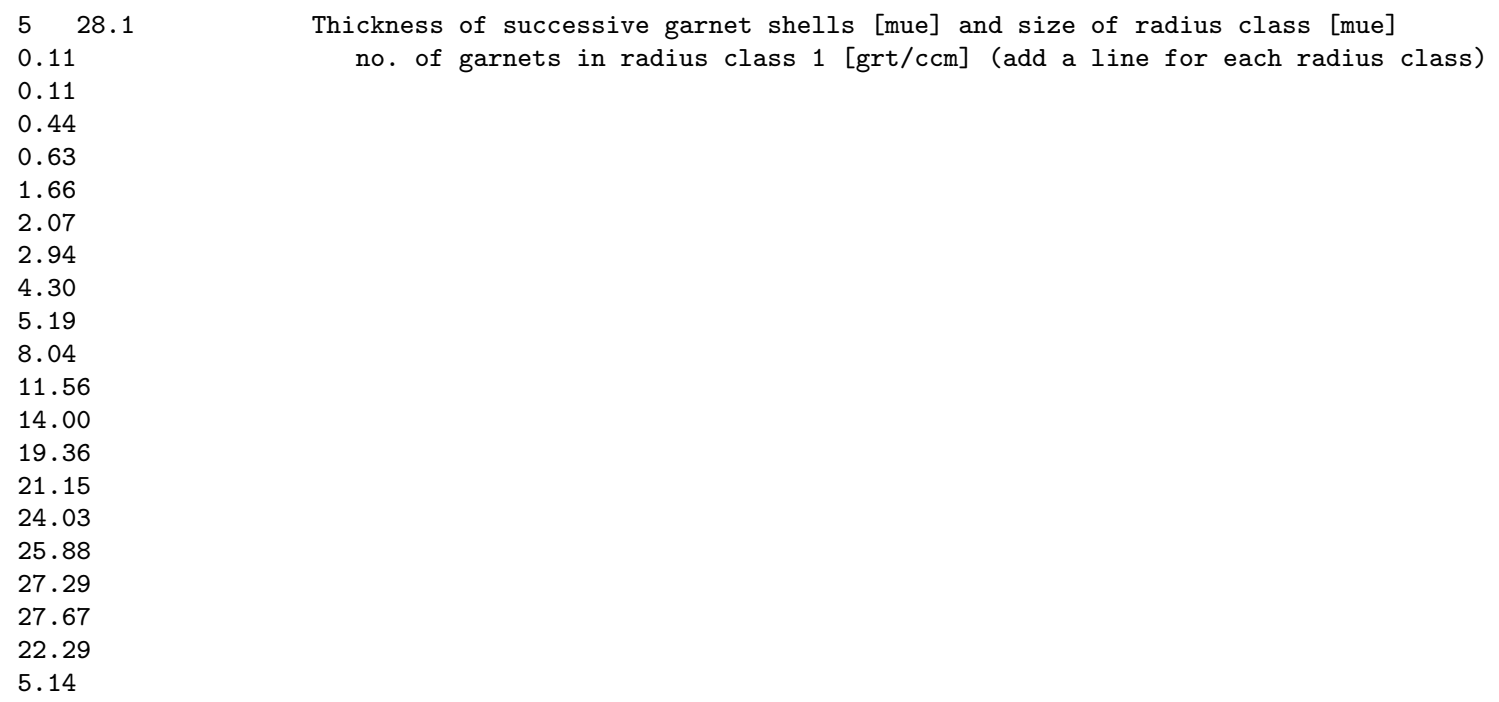

theriag_DIF.txt:

Kinetic parameters from Chakraborty and Ganguly (1992) at f02 defined by graphite buffer

5.1D-4 60569.0D0 6.0D0 MN: pre-exponential constant, activation energy, activation volume

6.4D-4 65824.0D0 5.6D0 FE: pre-exponential constant, activation energy, activation volume

1.1D-3 67997.0D0 5.3D0 MG: pre-exponential constant, activation energy, activation volume

theriag_PTt.txt:

$\begin{array}{llll}515 & 4300 & 0 & T \text { [degree C], P [bar] and } t \text { [my]. (t starts at zero) } \\ 525 & 4600 & 0.1 & \\ 542 & 5000 & 0.27 & \% \text { HR100:CR100 } \\ 550 & 5200 & 0.35 & \\ 555 & 5300 & 0.40 & \\ 563.96 & 5590 & 0.49 & \\ 565 & 5000 & 0.5 & \\ 535 & 3000 & 0.8 & \end{array}$

